\title{
Numerical simulations of wet granular matter
}

\author{
Dissertation \\ zur Erlangung des mathematisch-naturwissenschaftlichen Doktorgrades \\ "Doctor rerum naturalium" \\ der Georg-August-Universität Göttingen
}

vorgelegt von

Klaus Walter Röller

aus Ehingen

Göttingen, 2010 
$\mathrm{D}_{7}$

Referent: Prof. Dr. Stephan Herminghaus

Korreferentin: Prof. Dr. Annette Zippelius

Tag der mündlichen Prüfung: 26. April 2010 
"Don't say you don't have enough time. You have exactly the same number of hours per day that were given to Helen Keller, Pasteur, Michaelangelo, Mother Teresa, Leonardo da Vinci, Thomas Jefferson, and Albert Einstein."

(H. JACKSON BROWN) 



\section{Contents}

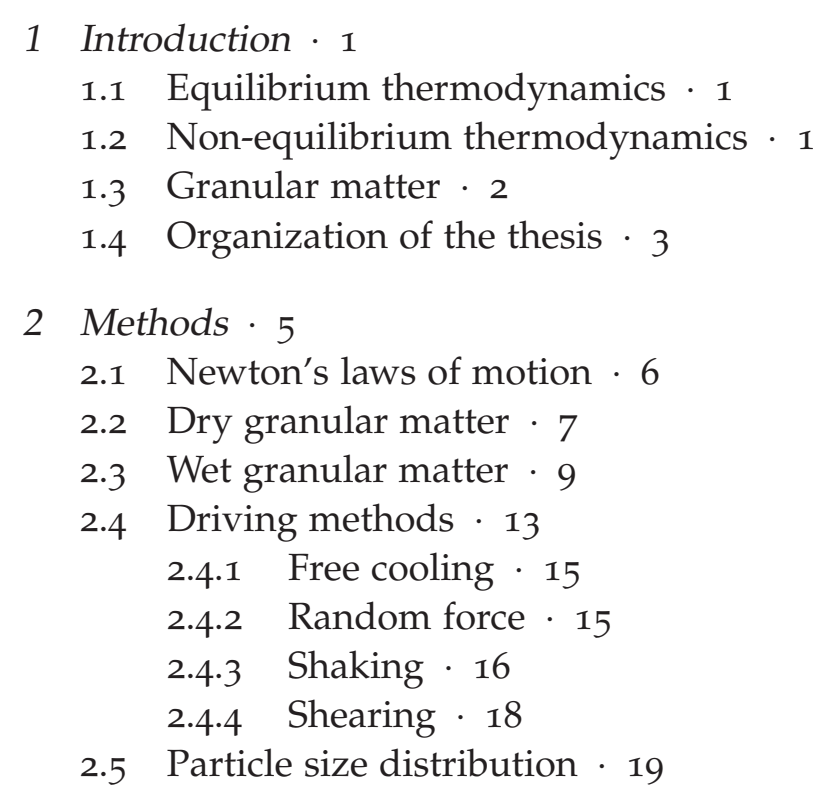

3 Phase diagrams of wet granular matter - sinusoidal shaking · 21

3.1 Elastic collisions - 22

3.1.1 Numerical setup · 22

3.1.2 Stationary states $\cdot 23$

3.1.3 Elastic phase diagrams $\cdot 25$

3.2 Theoretical description · 29

3.3 Inelastic collisions and comparison with experiments $\cdot 31$

3.3.1 Experimental setup $\cdot 31$

3.3.2 Results and discussions · 32

3.3.2.1 Observations in experiments $\cdot 32$

3.3.2.2 Phase diagram: experiments vs. simulations $\cdot 33$

3.3.2.3 Scaling of the $\mathrm{f}-\mathrm{fgc}$ transition - 37

3.3.2.4 Scaling of the fgc-g transition * 41

3.3.2.5 Narrower particle size distribution $\cdot 42$

3.3.2.6 Effect of inelasticity in dilute systems $\cdot 43$

3.4 Summary and Outlook $\cdot 52$ 
3.A Order parameters $\cdot 53$

3.A.1 Discrimination of homogeneous phases $\cdot 53$

3.A.1.1 Solid-fluid phase boundary $\cdot 53$

3.A.1.2 Fluid-gas phase boundary · 54

3.A.1.3 Solid-gas phase boundary $\cdot 56$

3.A.2 Discrimination of inhomogeneous phases $\cdot 56$

4 The solid-fluid phase transition and surface melting · 59

4.1 Introduction 59

4.2 Theoretical model · 59

4.3 Comparison with simulations $\cdot 62$

4.3.1 Scaling of the solid-fluid transition line $\cdot 62$

4.3.1.1 Dependence on the capillary bridge force $\cdot 63$

4.3.1.2 Dependence on the driving energy $\cdot 63$

4.3.2 Minimum value of the critical peak acceleration · 64

4.3.3 Surface melting $\cdot 64$

4.4 Summary and Outlook · 65

5 Interfacial energy in wet granular matter . 69

5.1 Introduction 69

5.2 Gas phase growth in fluid-gas coexistence $\cdot 70$

5.2.1 Experiments $\cdot 71$

5.2.2 Simulations $\cdot 72$

5.3 Interfacial energy $\cdot 79$

5.3.1 Observation $\cdot 79$

5.3.2 Estimation of the interfacial energy in simulations $\cdot 79$

5.3.3 Estimation of particle mobility $\cdot 87$

5.4 Coarsening dynamics $\cdot 91$

5.4.1 Nucleation and coalescence $\cdot 92$

5.4.1.1 Observation $\cdot 92$

5.4.1.2 Discussion of existing models · 96

5.4.2 Ostwald ripening $\cdot 98$

5.5 Anomalous diffusion in fluid-gas coexistence $\cdot 101$

5.5.1 Observation $\cdot 102$

5.5.2 Results · 104

5.6 Summary and Outlook · 106

6 The granular Leidenfrost effect $\cdot 109$

6.1 Introduction - 109

6.1.1 Classical Leidenfrost effect with water - 109

6.1.2 Leidenfrost effect in dry granular matter . 110

6.2 Leidenfrost effect in wet granular matter · 111

6.3 Vertical plug oscillation · 112 
6.4 Theoretical model $\cdot 117$

6.4.1 Passive plug on gas layer · 117

6.4.2 Plug on a gas layer with exchange of particles · 119

6.4.3 Plug on a gas layer with exchange of particles and a temperature gradient $\cdot 120$

6.4.4 The complete picture, with lateral gas exchange $\cdot 122$

6.5 Comparison of theory and simulations $\cdot 125$

6.5.1 Plug oscillation frequency $\cdot 125$

6.5.2 Bubbling in wet granular matter $\cdot 126$

6.6 Summary and Outlook · 128

6.A Inelasticity damps plug oscillation · 130

7 Phase diagrams of wet granular matter - sinusoidal shearing $\cdot 133$

7.1 Introduction · 133

7.2 Fluidization point of sheared wet granular matter $\cdot 134$

7.3 Phase diagrams 139

7.4 Summary and Outlook $\cdot 144$

8 Unstable Kolmogorov flow in granular matter · 147

8.1 Introduction - 147

8.2 Bifurcation diagram $\cdot 148$

8.3 Phase space plots - Limit cycles · 149

8.4 Poincaré maps · 155

8.5 Summary and Outlook · 156

9 Summary 159

Appendices $\cdot 163$

A Velocity distribution of a wet granular gas $\cdot 165$

A.1 The Boltzmann equation for a wet granular gas $\cdot 165$

A.1.1 Modeling a wet granular gas $\cdot 165$

A.1.2 Collision rules 166

A.1.3 A useful property of the collision integral $\cdot 167$

A.2 Velocity distribution expansion $\cdot 167$

A.2.1 Normalized velocity distribution 168

A.2.2 Laguerre polynomial expansion · 168

A.2.3 Moments of the Boltzmann equation - 170

A.2.3.1 Second moment · 171

A.2.3.2 Fourth moment $\cdot 172$

A.2.4 Result $\cdot 173$ 
B Sinusoidal shaking in event-driven simulations · 175

B.1 Introduction - 175

B.2 The mathematical task $\cdot 176$

B.2.1 Calculation of the collision time - 177

B.2.1.1 Particle-particle collision · 177

B.2.1.2 Particle-wall collision 177

B.2.2 Two reasonable choices for the frame of reference $\cdot 178$

B.3 A first algorithm $\cdot 178$

B.3.1 Back to the roots $\cdot 178$

B.3.2 Search for unique bracketing bounds - 179

B.3.3 Assure termination of the algorithm - 180

B.3.3.1 The starting point $\cdot 180$

B.3.3.2 The exception - 181

B.4 Improving the efficiency with look up tables $\cdot 183$

B.4.1 What to store in the table $\cdot 183$

B.4.2 Trajectories as parameters of the table $\cdot 184$

B.4.3 The mapping of velocities · 186

B.4.4 Extrapolation of table entries - 186

B.4.5 Selection of the appropriate root $\cdot 187$

B.4.6 Precision and applicability $\cdot 188$

B.4.7 Initialization of the look up table $\cdot 189$

B.5 Essential numerical improvements · 189

B.5.1 Particle positions · 189

B.5.2 Extra table after wall collisions - 190

B.5.3 Tabulation of the relative velocity - 190

B.5.4 Extrapolation across discontinuities · 191

B.5.5 Assuring a consistent description between collision and escape $\cdot 192$

B.5.6 Instantaneous events as protection against unphysical behavior - 193

B.6 Comparison of performance · 194

B.6.1 Comparison of the simple algorithm with tabulation · 194

B.6.2 Comparison of overall performance · 194

B.7 Summary · 195

Bibliography $\cdot 199$

List of publications $\cdot 217$

Zusammenfassung in deutscher Sprache · 219

Acknowledgements $\cdot 223$

Awards and scientific meetings $\cdot 225$

Curriculum Vitae $\cdot 227$ 


\section{Introduction}

\subsection{Equilibrium thermodynamics}

Equilibrium thermodynamics is a branch of physics which evolved in the $18^{\text {th }}$ and $19^{\text {th }}$ centuries based on the work of D. Bernoulli, B. Thompson and J. Joule (Vogel, 1999). It deals with the energy of systems, its transformation from heat to work and vice versa. The field has been continuously developed since then and has proven to be a useful tool for many applications in technical sciences, physics, chemistry, biology and other disciplines.

Equilibrium thermodynamics predicts the behavior of physical systems only by considering their macroscopic state functions (such as entropy, temperature, pressure and volume). A system is said to be in equilibrium if there are no time-dependent quantities in the system, such that very slow changes, as in quasistatic processes, are considered a succession of equilibrium states.

However, as will be seen in the following section, many systems of interest are not in equilibrium. Hence, in the $19^{\text {th }}$ and $20^{\text {th }}$ centuries, a new branch of thermodynamics, called non-equilibrium thermodynamics, emerged. It aims at developing tools that provide a description of processes beyond the scope of equilibrium thermodynamics.

\subsection{Non-equilibrium thermodynamics}

Non-equilibrium systems can be found in almost all scientific disciplines ranging from chemistry (e.g. the Belousov-Zhabotinsky reaction in Belousov (1959, 1951) and Zhabotinsky $(1964,2007))$, to quantum field theory (for example Rammer, 2007) and information theory (for example Andrews et al., 2008). A thermodynamic system is out of equilibrium if the system is time-dependent. This appears in systems which are either open or dissipative. Due to the importance of mass or energy flux through the system's boundaries, it is not possible to apply the thermodynamic limit in this case. Indeed, non-equilibrium systems are very commonly encountered, and are familiar to the non-scientist as well as the scientist. This is visible, for instance, in self-organised systems (Haken, 2008), like the formation of snow crystals or the stripes on zebras, and all transport phenomena, like opening a window to air a room 
or placing a pot of water on the stove.

The formulation of the Boltzmann equation in 1896 was the first major step in non-equilibrium statistical physics (see also Brilliantov and Pöschel, 2004). It describes the time evolution of the distribution function to find a particle with velocity $\vec{v}$ at a position $\vec{q}$, and can be used to calculate material properties, like heat conductivity or viscosity. It is based on the assumption of molecular chaos, which states that particle velocities are uncorrelated before collisions and can be described by only considering two-particle interactions. Correlations between collisions can be taken into account by solving the BBGKY (Bogoliubov-Born-Green-Kirkwood-Yvon) hierarchy (Bogoliubov, 1946; Bogoliubov and Gurov, 1947; Born and Green, 1946; Kirkwood, 1946, 1947; Yvon, 1935).

The next major advances in non-equilibrium statistical mechanics were the Einstein kinetic theory of Brownian motion (Einstein, 1905) and the Onsager reciprocal relations (1931). In the 1950's, Green and Kubo derived an expression for the linear transport coefficients and showed how they can be calculated from fluctuations (Green, 1954; Kubo, 1957) for systems which are close to equilibrium. The most recent major progress is the Gallavotti-Cohen fluctuation theorem (Gallavotti, 2008), which was first observed by Evans et al. (Evans et al., 1993a,b) and later proved by Gallavotti and Cohen (1995) for dynamic systems, and for Markov processes by Kurchan, Lebowitz and Spohn (Kurchan, 1998; Lebowitz and Spohn, 1999). The fluctuation theorem is equivalent to the second law of thermodynamics when applied to macroscopic systems. In non-equilibrium thermodynamics it is convenient to investigate steady states since the complexity of the physical situation is reduced due to the absence of an explicit time dependence (Evans et al., 1993b). Nevertheless, the understanding of non-equilibrium systems is far from being as complete as for equilibrium systems. Many interesting systems lie far beyond the scope of what is addressable with the concepts mentioned above. The present thesis aims to contribute to the field of non-equilibrium statistical physics.

\subsection{Granular matter}

Granular matter is an accumulation of solid particles, where each particle is of macroscopic size, starting with grains of a few microns and ranging up to ice floes floating across the polar seas (Duran, 2000). Granular matter is ubiquitous in daily life. After getting up, you find it as cereals on the breakfast table and as abrasive particles in toothpaste. Later on in the office you are sitting at the computer, which expends energy produced in a coal-burning power plant, and after work you return to your home, which has an environmentally friendly woodchip-fired central heating. Finally, after eating rice with meatballs, potatoes, peas and carrots for dinner, you nibble some snacks like peanuts or grapes before going to bed. During your vacation you go to the beach where you build impressive sand sculptures out of the wet sand. We are surrounded by granular materials all day long. And these were 
only examples where the granular character was still present in the final product. Sand, for example, is used as a raw material in the production of concrete, glass, porcelain or silicon. Therefore, it is not surprising that the processing of granular materials and aggregates consumes about $10 \%$ of the total worldwide energy production (Duran, 2000). Understanding of the dynamical behavior, of granular matter or bulk solids, is thus not only of scientific, but also of economic interest.

Due to the large mass of individual grains ${ }^{1}$, the random motion of agitated granular particles corresponds to temperatures ${ }^{2}$ of Giga- or even Terakelvin. Through interactions between grains (like inelastic collisions between particles or rupturing of capillary bridges between wet grains) this random motion is coupled to a heat bath of the atomic degrees of freedom (of the particles or of the liquid of the capillary bridges) which is at room temperature. The vast temperature difference, between particle motion and the heat bath, leads to a strong non-equilibrium situation. Granular matter is thus one of the most widespread systems which exhibit nonequilibrium behavior and, therefore, it is the system studied in this thesis.

\subsection{Organization of the thesis}

The scope of this work is to study wet granular matter under sinusoidal driving with the help of numerical simulations. It is organized as follows. Following the introductory chapter 2, the methods used to model granular materials and different driving mechanisms to allow a numerical study are briefly introduced. Two different sinusoidal driving mechanisms are used in this thesis, which are the natural counterpart of each other. Former, sinusoidal shaking in the chapters 3 to 6 , and latter, sinusoidal shearing in the chapters 7 and 8 .

The phase diagram as obtained by sinusoidal shaking is shown and analyzed in great detail in chapter 3. The following chapters (4 to 6) each focus on a special region of the phase diagram and study the emerging dynamics. In chapter 4 , the solid-fluid transition is studied in more detail and reveals surface melting. In chapter 5, the lateral phase separation is studied and suggests interfacial energy in the coexistence states. Finally in chapter 6 , a phase separation in the vertical direction is observed and its resemblance to the classical Leidenfrost effect is discussed.

Similarly for sinusoidal shearing, the different phase diagrams are presented in chapter 7 , followed by chapter 8 , which studies the complex dynamics occuring in the fluidized state. The thesis is summarized in chapter 9 .

In the appendix $\mathrm{A}$ a derivation of an analytic expression for the velocity distribution in a wet granulate using a Laguerre polynomial expansion is shown. This is

1. Compared to the mass of a single molecule.

2. Temperature, $T_{g}$, (often called granular temperature) is defined as the kinetic energy of the random motion of the granular particles. $T_{g}:=m\left(\left\langle\vec{v}^{2}\right\rangle-\langle\vec{v}\rangle^{2}\right) / k_{B} f_{d}$ (Ogawa et al., 1980) where $m$ is the average mass of an individual particle with velocity $\vec{v}, k_{B}=1$ is 'Boltzmann's constant' and $f_{d}$ the number of degrees of freedom. 
followed in appendix $B$ by a numerical method which efficiently implements sinusoidally moving walls in event-driven simulations (cf. list of awards on page 225). A list of publications to which the author of the present thesis contributed can be found at the end of this document on page 217. 


\section{Methods}

As in most non-equilibrium systems, it is challenging in the case of granular matter to obtain analytical results: much of the theoretical work in this field uses strong simplifications like considering only one-dimensional systems (Zaburdaev et al., 2006; Fingerle and Herminghaus, 2006; Zaburdaev and Herminghaus, 2007) or very dilute systems (Brilliantov and Pöschel, 2004). Quantitative experiments can be expensive or laborious and even then it can be difficult to attribute certain phenomena to the properties of the physical system. Therefore, numerical simulations present an opportunity to gain insight into systems which elude analytical investigation at first and give access to more information than experimental studies. Therefore, granular matter out of equilibrium is studied in this thesis by means of numerical simulations.

Two different approaches a continuum and a discrete element method for modeling granular matter in numerical studies are distinguished.

The continuum method only considers fields and not individual particles. It was inspired by computational fluid dynamics for classical liquids, where the discrete molecular structure is also disregarded. In the context of granular matter, this method is sometimes called 'Navier-Stokes granular hydrodynamics' or 'ideal granular hydrodynamics' (Meerson et al., 2003; Khain and Meerson, 2003; Puglisi et al., 2008).

The discrete element methods can be divided into different categories. Firstly, the Monte Carlo method is a stochastic simulation method completely based on random numbers and repeatedly predicts the physical behavior of a system (Allen and Tildesley, 1987; Poeschel and Schwager, 2004; Brilliantov et al., 2007). Secondly, the method of cellular automata, the particle positions are discretized such that particles are located at fixed sites as on a lattice. Exchange between sites is realized by defining transition rates between neighboring sites. This method is used for example in lattice gases (Hardy et al., 1973; Frisch et al., 1986). Thirdly, the simulation method of granular dynamics, is mostly called molecular dynamics simulation in literature, due to its historical origins. In this thesis this historical name (Poeschel and Schwager, 2004) is also used. It is stressed that the notion 'molecular' is misleading in the context of granular matter, because the molecular degrees of freedom are not considered at all. The molecular structure is rather reflected in a simple model such that it is sufficient to consider the macroscopic structure of the particles. The dynamics in this method are governed by Newton's laws of motion, which are then 
solved numerically.

In this thesis solely discrete element simulations using the molecular dynamics simulation method are performed and, wherever possible, compared with experimental results.

In the remainder of this section the most important ingredients are mentioned to model dry and wet granular matter in the framework of molecular dynamics simulations, and two different simulation techniques which can physically be interpreted as hard and soft particles are distinguished. The hard sphere method was originally developed by Alder and Wainwright (1957, 1959) and the soft sphere method was originally developed by Cundall and Strack (1979). For details on the realization of these methods see Poeschel and Schwager (2004), Zhu et al. (2007) and references therein.

\subsection{Newton's laws of motion}

The dynamics of granular particles, in molecular dynamics simulations, is fully governed by Newton's laws of motion. In this thesis only the translational degrees of freedom of the granular particles are considered. Disregarding the rotational degrees of freedom is admissible as long as there is no friction between particles. See Poeschel and Schwager (2004), Zhu et al. (2007) and Brilliantov et al. (2007) and references therein for a discussion of tangential (frictional) forces.

In the following forces which occur while particles are in contact and those which do not require a contact, i. e., act even over a distance are treated seperately. The equation of motion for the translational motion of particle $i$, with mass $m_{i}$ then reads

$$
m_{i} \frac{\mathrm{d} \vec{v}_{i}}{\mathrm{~d} t}=\sum_{j} \vec{F}_{i j}^{c}+\sum_{k} \vec{F}_{i k}^{\mathrm{nc}}+\vec{F}_{i}^{g}+\vec{F}_{i}^{\mathrm{driv}}
$$

where $v_{i}$ is the translational velocity of particle $i, \vec{F}_{i j}^{c}$ are the contact forces asserted by particle $j$ on particle $i, \vec{F}_{i k}^{\text {nc }}$ are the non-contact forces on particle $i$ caused by particle $k$ or other components in the system (like a wall), $\vec{F}_{i}^{g}=m_{i} \vec{g}$ is the gravitational force acting on particle $i$ with the acceleration due to gravity $\vec{g}$ and $\vec{F}_{i}^{\text {driv }}$ is the external driving force which acts on particle $i$.

There are many different forces which can act on and impose a certain physical behavior on granular particles in different physical situations (Zhu et al., 2007). Examples of contact forces are the elastic or dissipative repulsive forces while noncontact forces are capillary, electrostatic, or Van der Waals forces. These forces can be used in different combinations resulting in a large number of possible models for granular matter. In the following, solely the models for (dry and wet) granular matter, which are used in this thesis or which are typically studied in the literature, are presented. 


\subsection{Dry granular matter}

This thesis is restricted to particles of idealized shape which are circular disks (in two-dimensional modeling) and spheres (in three-dimensions). This is not a serious constraint, because particles of completely irregular shape can be approximated by an accumulation of spherical particles (Bertrand et al., 2005). Therefore, it is sufficient to have a model for the contact forces of spherical particles only. As mentioned before, molecular degrees of freedom of individual particles are neglected in the simulation, but therefore a (preferably simple) model, which accounts for forces arising through the molecular interaction when two particles collide, is needed. These forces can be decomposed into two components. First, the elastic deformation of the particles which is responsible for the repulsion, and second, the dissipative energy loss, which accounts for the energy transfer into the molecular degrees of freedom. Different types of models for colliding particles like the linear spring-dashpot model, Walton and Braun's model, the Hertz model or the hard core model have been proposed (Zhu and Yu, 2006; Zhu et al., 2007; Poeschel and Schwager, 2004) of which different ones are used in this thesis.

The first one is the hard-core interaction force which assumes an infinite force if the particles are overlapping. It reads

$$
F_{\mathrm{hc}}(\xi)=\left\{\begin{aligned}
0 & \text { for } \quad \xi<0 \\
\infty & \text { for } \xi \geqslant 0
\end{aligned}\right.
$$

where $\xi=R_{i}+R_{j}-r_{i j}$ is the overlap between particles ${ }^{1}$ and $r_{i j}$ is the distance between the particle centers. Due to the infinite force, this model represents ideal hard particles. Inserting Eq. (2.2) into the equation of motion, Eq.(2.1) is directly solvable analytically and the particle trajectories in-between collisions are obtained. Thus, an event-driven algorithm to simulate this model can be used. For more detail on the algorithm see Appendix $B$ and Poeschel and Schwager (2004). Because the underlying differential equation is solvable analytically, this approach is preferred whenever possible. For identical particles, the collision rules, for the exchange of the momentum through a collision, read

$$
\begin{aligned}
& \vec{v}_{i}=\vec{v}_{i}^{* *}-(1+\varepsilon) \frac{\vec{\sigma}}{2}\left(\vec{v}_{i j}^{* *} \vec{\sigma}\right) \\
& \vec{v}_{j}=\vec{v}_{j}^{* *}+(1+\varepsilon) \frac{\vec{\sigma}}{2}\left(\vec{v}_{i j}^{* *} \vec{\sigma}\right),
\end{aligned}
$$

where $v_{i \mid j}$ and $v_{i \mid j}^{* *}$ is the velocity of particle $i \mid j$ after and before the collision respectively, while $\vec{v}_{i j}^{* *}$ is the relative velocity between particles $i$ and $j$ before the collision. At the time instant of the collision, the unit vector $\vec{\sigma}$ points from the center of particle $j$ to the center of particle $i$. In this model dissipation is introduced by a coefficient of

1. It is noted that $\xi$ is positive for overlapping particles. 
restitution, $\varepsilon$, which is defined as

$$
\varepsilon:=\frac{p_{f}}{p_{i}}
$$

where $p_{i}$ and $p_{f}$ are the momenta before and after the collision respectively. This coefficient depends in general on the impact velocity, $v_{\text {imp }}$, as $\varepsilon\left(v_{\text {imp }}\right) \approx 1$ - const. . $v_{\text {imp }}^{1 / 5}$ (Schwager and Pöschel, 1998; Ramírez et al., 1999; Brilliantov and Pöschel, 2001) and approaches $\varepsilon \approx 1$ for impact velocities going to zero. For the simulations, a constant (i. e. velocity independent) coefficient of restitution is used, because it is almost constant in the range of impact velocities considered in this thesis. A characteristic quantity in this model is the dissipated energy per collision, which is

$$
\Delta E_{\text {diss }}=E_{i}\left(1-\varepsilon^{2}\right),
$$

where $E_{i}$ is the kinetic energy before the collision. In Giese and Zippelius (1996), Gerl and Zippelius (1999), Kuninaka and Hayakawa (2004) and Schwager (2007) how the coefficient of restitution arises out of the molecular degrees of freedom of the particles has been carefully studied.

If the soft sphere model is used ${ }^{2}$ it is not always possible to solve Eq. (2.1) analytically. In this case, one has to rely on a time-driven integration method to solve the equation of motion numerically. In the present study the Gear integration scheme is used, which has proven to be successful for granular matter (Poeschel and Schwager, 2004). Because the time-driven simulation method cannot deal with infinite forces, the Hertz contact force is used instead which showed good agreement with experiments (Ramírez et al., 1999). It acts normally to the particle surface, and for non-identical particles it reads

$$
F_{\text {Hertz }}(\xi)=\frac{2 Y_{m} \sqrt{R_{\text {eff }}}}{3\left(1-v_{p}^{2}\right)}\left(\xi^{\frac{3}{2}}+A_{d} \sqrt{\xi} \frac{\mathrm{d} \xi}{\mathrm{d} t}\right)
$$

where $Y_{m}$ is the Young modulus, $R_{\text {eff }}=\frac{R_{i}+R_{j}}{R_{i} R_{j}}$ is the effective radius of spheres $i$ and $j$ with radii $R_{i}$ and $R_{j}$ respectively; $v_{p}$ is the Poisson ratio, $\xi$ is the overlap of the two particles and $A_{d}$ is the dissipative constant. The first term in the formula is the elastic repulsion, while the second term accounts for the dissipative damping. In this model the particles interact through a finite overlap, which is interpreted as slight particle deformation. Consequently, they are called soft particles.

2. The hard-core model should be used wherever possible, due to its precision and efficiency. The soft sphere model is, however, preferred in very dense systems, when it becomes important to account for a finite time two particles are in contact during a collision, or if there are additional non-contact forces in the system which prevent analytical solvability of the equations of motion like for the $n$-body problem. 
Dry granular matter ${ }^{34}$ is thoroughly studied in the literature (Jaeger et al., 1996; Zhu et al., 2008, and references therein).

\subsection{Wet granular matter}

There are several, mostly cohesive, forces, like Van der Waals forces, electrostatic forces, gravitational forces or capillary bridge forces, which can act between granular particles even if they are not in contact. A comparison of the magnitude of these different forces is shown in Fig. 2.1. It is obvious that the strongest inter-particle force is the capillary bridge force and it therefore has a huge influence on the physical behavior of granular matter. In contrast to powders or dry granular matter, only a comparatively small amount of work has been devoted to wet granular matter (Hornbaker et al., 1997; Bocquet et al., 2002; Nowak et al., 2005; Herminghaus, 2005, and references therein) or (Scheel et al., 2008), thus the focus lies on wet granular matter in this thesis.

In Fig.2.2 the formation process of a capillary bridge is schematically seen, which forms between two identical spheres, wetted by a thin liquid film (Kohonen et al., 2004), when they collide: The liquid accumulates around the contact point. The capillary bridge exerts a certain attractive force, $F$, on the particles, which is caused by the surface tension of the wetting liquid. If the particles separate again, the bridge narrows and finally ruptures at a critical surface separation distance (sometimes also called rupture distance), $s_{\text {crit }}$. This force and its dependence on the surface separation, s, was experimentally measured in Willett et al. (2000). A typical outcome of such a measurement is shown in Fig. 2.3. It can be observed that the force decreases when the separation between particles increases and vanishes abruptly when the capillary bridge ruptures at a certain surface separation. Since the two curves belong to two different volumes of the capillary bridge, it can be seen that the rupture distance is a function of the capillary bridge volume.

3. When studying very small and fine particles, the cohesive Van der Waals force becomes increasingly important when compared to the gravitational force and must be included in the model. This physically represents powders. Powders like flour, dust and many pharmaceutical products can form clumps due to this additional cohesive force (Dhanarajan and Bandyopadhyay, 2007). Even if this model is not as thoroughly studied as the dry granular matter there is quite a body of work in this field (Duran, 2000; Garcia-Rojo et al., 2005).

4. The Van der Waals gas does not belong to the group of granular matter. Nevertheless it uses a model for its molecular interaction very similar to the one discussed above for macroscopic grains. Atoms are modeled in this framework as elastically colliding spheres with (in contrast to the ideal gas) finite volume. In terms of the forces discussed above, this means that the particles are modeled by a repulsive hard-core force while the collision itself is elastic $(\varepsilon=1)$. Even if the physical system is very different, from the numerical point of view, the Van der Waals gas can be viewed as the most simple realization of a model using the same ingredients as those in granular matter research. Therefore, it is not surprising that in granular materials there are also attempts in the literature to find an equation of state (Fingerle and Herminghaus, 2008; Herbst et al., 2004) reminiscent of the Van der Waals equation, even though granular matter is not an equilibrium system. 


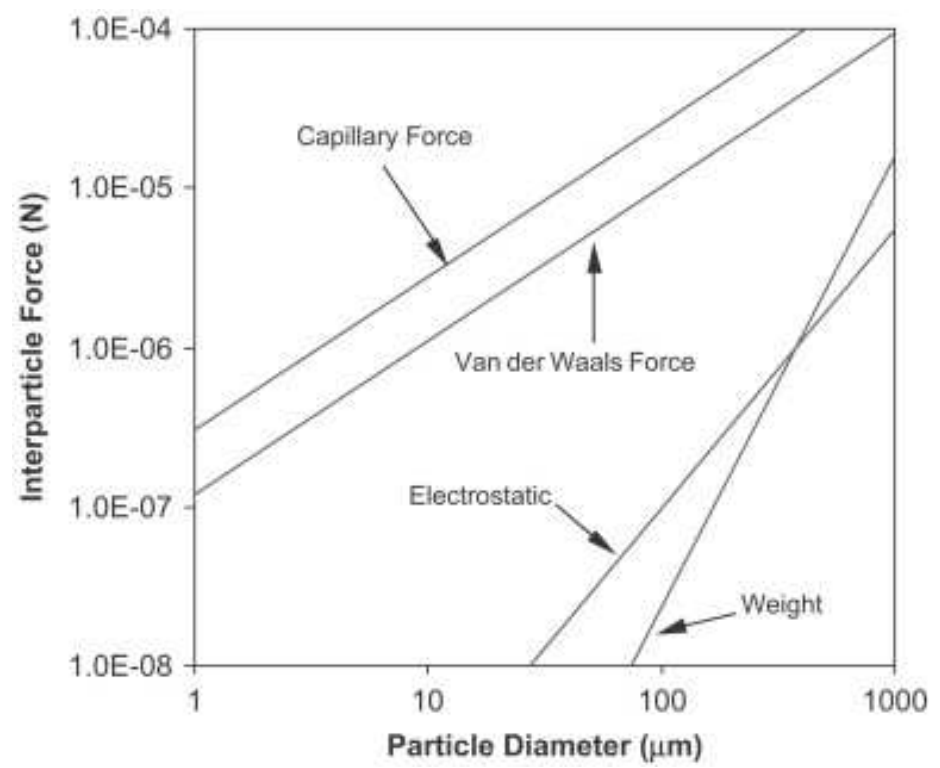

Fig. 2.1 The magnitude of several inter-particle forces. The calculations were based on the following approximated equations and values: (i) capillary force for particles in contact (surface tension): $\vec{F}_{B}=$ $-2 \pi \gamma R \cos \theta_{c} \hat{n}_{i j}$ with $\gamma=72.8 \times 10^{-2} \mathrm{Nm}^{-1}$ (water at $20^{\circ} \mathrm{C}$ ), (ii) Van der Waals force (molecular dipole interaction): $\vec{F}_{\mathrm{vdW}}=-\frac{A d_{p}}{24 h^{2}} \hat{n}_{i j}$ with $A=6.5 \times 10^{-20} \mathrm{~J}, h=1.65 \AA$, (iii) electrostatic force (Coulomb force): $\vec{F}_{e}=-\frac{Q^{2}}{16 \pi q_{0} h^{2}}\left(1-\frac{h}{\sqrt{R^{2}+h^{2}}}\right) \hat{n}_{i j}$ with $\varepsilon_{r}=1$, and (iv) weight: $\vec{F}_{g}=m \vec{g}$ with $\rho_{g}=3 \times 10^{3} \mathrm{~kg} / \mathrm{m}^{-3}$. Graph reprinted from Rumpf (1962) and Zhu et al. (2007) with permission from Elsevier.

The force, $F_{B}$, which is caused by the capillary bridge can be calculated analytically for the simplest case of two identical, spherical particles being in contact. This was first done as long ago as 1926 (Fisher, 1926). The capillary bridge force for particles at contact, in lowest order approximation is

$$
F_{B}=2 \pi \gamma R \cos \theta_{c}
$$

where $\gamma$ is the surface tension of the liquid, $R$ is the radius of the (identical) spheres and $\theta_{c}$ is the contact angle between the wetting liquid and the sphere (Princen, 1968; Lian et al., 1993; Willett et al., 2000; Herminghaus, 2005; Butt and Kappl, 2009). For finite separation distances between the particle surfaces the capillary bridge force is well approximated by

$$
F=\frac{F_{B}}{1+1.05 \hat{s}+2.5 \hat{s}^{2}}
$$

with $\hat{s}:=s \sqrt{R / V}$, where $V$ is the volume of a single capillary bridge (Willett et al., 2000). As mentioned before, the critical surface separation for the capillary bridge to rupture depends on the volume of the capillary bridge. As derived in Willett et al. 
a

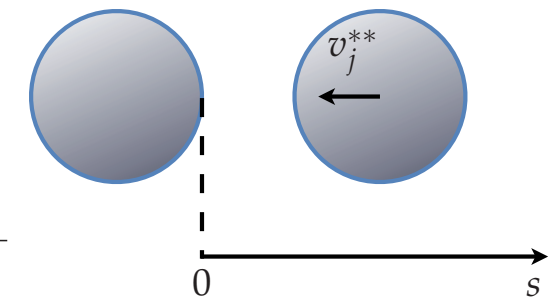

C

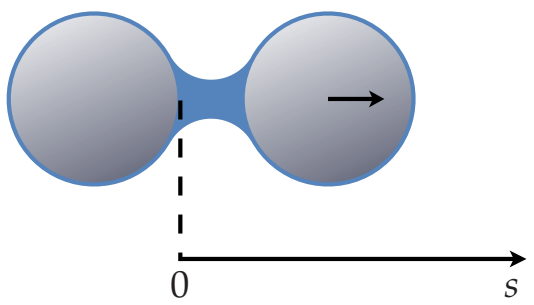

b

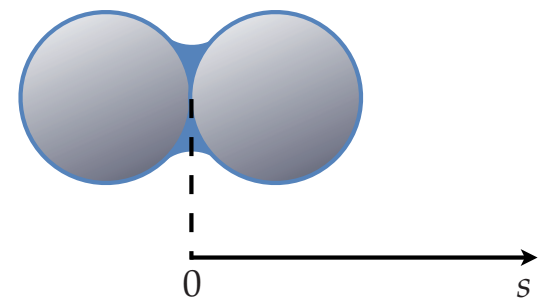

d

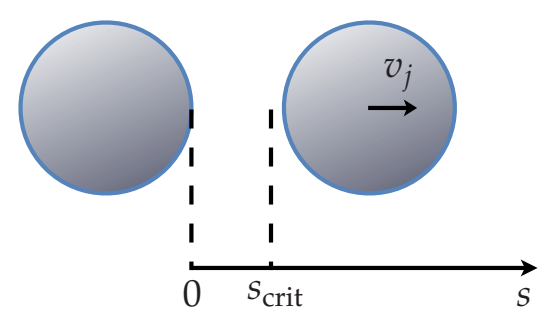

Fig. 2.2 Schematic process of capillary bridge formation between two spherical particles which are covered by a thin liquid film. In a particle $j$ is approaching a (which is assumed to be at rest) with velocity $v_{j}^{* *}$ and where $s$ is the surface separation. Particles collide in $\mathbf{b}$ and the liquid accumulates (instantaneously, in the model used) around the contact point of the two particles and forms a capillary bridge. Due to the collision the particles separate again, as seen in $\mathbf{c}$, and the capillary bridge narrows until it ruptures at a critical surface separation, $s_{\text {crit }}$, as can be seen in $\mathbf{d}$. Due to the hysteretic, attractive force which is exerted by the capillary bridge, some energy is dissipated so that the velocity $v_{j}$ after the rupturing is smaller than before, i. e. $v_{j}<v_{j}^{* *}$.

(2000), a good approximation of this dependence, which is valid for small rupture distances, is

$$
\tilde{s}_{\text {crit }}=\left(1+\frac{\theta_{c}}{2}\right)\left(\tilde{V}^{1 / 3}+0.1 \tilde{V}^{2 / 3}\right),
$$

with the non-dimensionalized units $\tilde{s}_{\text {crit }}=s_{\text {crit }} / R$ and $\tilde{V}=V / R^{3}$. In Fig. 2.4, snapshots of an experiment (Ardekani and Rangel, 2008) are shown where the formation and elongation of a capillary bridge can be seen. The rupture distance in these snapshots is, however, much larger than the validity limit of Eq. (2.10), but rupture distances in the present work are restricted to values which are small compared to the average particle diameter.

Besides the capillary bridge force, $F$, and the critical surface separation, $s_{\text {crit }}$, where the capillary bridge ruptures, there is a third important quantity to characterize the capillary bridge interaction. This is the amount of energy which is dissipated through one complete cycle of the formation and rupture of a capillary bridge and is called the capillary bridge energy $E_{\mathrm{cb}}$. It reads

$$
E_{\mathrm{cb}}=\int_{0}^{s_{\text {crit }}} F(s) \mathrm{d} s .
$$




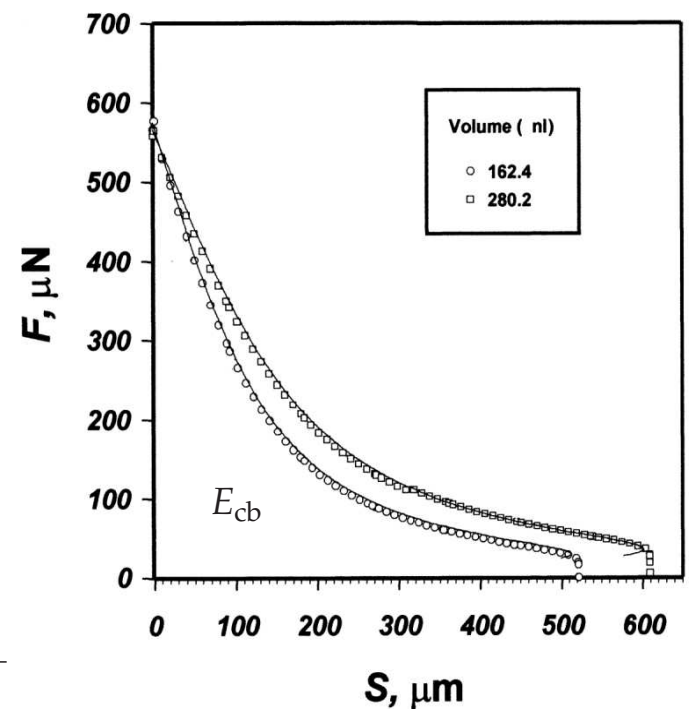

Fig. 2.3 Dependence of the capillary bridge force $F$ on the surface separation $s$ between a spherical particle and a flat wall as deduced from an experiment (circles) or numerical solution (straight line) of the Laplace-Young equation using perfect wetting $\left(\theta_{\mathcal{C}}=0\right)$. Graph reprinted from Willett et al. (2000) with permission.

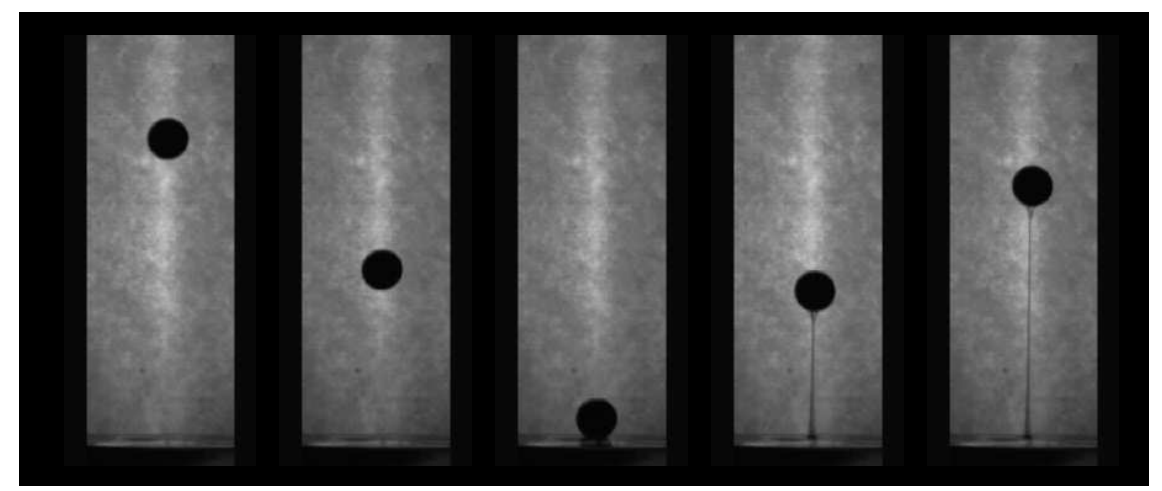

Fig. 2.4 Steel sphere (diameter $9.52 \mathrm{~mm}$ ) collides with a wall coated with a viscoelastic liquid $(1.5 \%$ polyethylene-oxide in water). During the collision a capillary bridge forms between the particle and the wall which can be elongated to a length much longer than the particle diameter. In the present thesis, however, rupture distances are restricted to values which are small compared to the average particle diameter. The time between subsequent snapshots is equal. Pictures are taken from the video accompanying Ardekani and Rangel (2008). 
Data presented in Fig. 2.3 are based on experiments demonstrating on how the interaction between grains is influenced by the presence of a capillary bridge exerting a certain force. Although this force and its dependence on the distance between the two particle surfaces has been studied carefully, it is not used in this in the present thesis in full detail. In fact, two different models are used in this work, which are both simplified versions of the real behavior.

The first model is the minimal capillary model (Herminghaus, 2005), where the capillary bridge force, $F_{B}$, is assumed to be independent of the surface separation $s$. This means that the capillary bridge force is a constant and therefore the capillary bridge energy simply reads $E_{\mathrm{cb}}=F_{B} S_{\text {crit. }}$. This is shown in Fig. $2.5 \mathrm{a}$, where also the hysteretic formation process of the capillary bridge is shown for a one dimensional collision, the red color denotes the existence of a capillary bridge. This model is numerically solved, in two and three dimensions, with soft, elastically colliding particles with capillary bridges using time-driven molecular dynamics simulations. Also a pseudo-potential representation is given in the respective figure to enhance the perceivability.

The second model, which is called thin-thread model, is inspired by the fact that in some cases only the capillary bridge energy $E_{\mathrm{cb}}$ is important, irrespective of the values $F_{B}$ or $s_{\text {crit }}$ (Fingerle et al., 2008). It is specially designed such that it can be simulated with an event-driven algorithm in any dimension and both elastic and inelastic collisions between spheres are used. This model is sketched in Fig. 2.5 b. The capillary bridge force is a delta distribution $F=\delta\left(s-s_{\text {crit }}\right)$, only acting at the critical surface separation, such that the area under the distribution gives the capillary bridge energy $E_{\mathrm{cb}}$. Here too, red indicates the existence of a capillary bridge and it can be seen that there is no force acting for surface separations smaller than the critical surface separation $s_{\text {crit }}$, even though a capillary bridge exists. For comparison with the minimal capillary model the mean acting force is calculated as $F_{B}=E_{\mathrm{cb}} / s_{\text {crit }}$. In the pseudo-potential representation this model looks like a square well potential.

As we will see in the next chapter, many physical aspects of the system do not depend on the detailed functional form of the underlying force. Therefore, it is justified to neglect the detailed dependence of the force on the surface separation, s. This suggests a certain universality for the investigated phenomena.

\subsection{Driving methods}

Because the energy of the random motion is dissipated, it is necessary to provide some driving to inject energy into the system in order to maintain the random motion of the granular particles. If the driving is switched off the particles will all come to rest as was experimentally studied by Son et al. (2008).

There are (also in nature) different ways through which the driving of a granular system can be realized. This can be done by injection of air (as in sand storms which 
a minimal capillary model
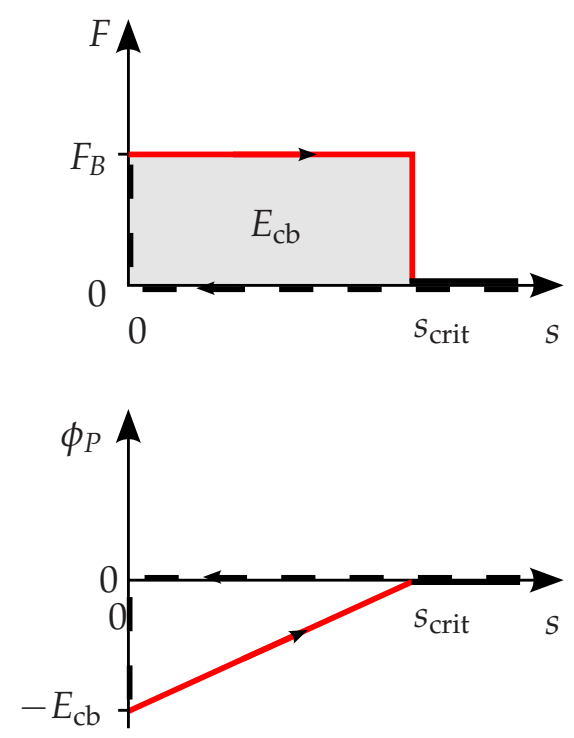

b thin-thread model
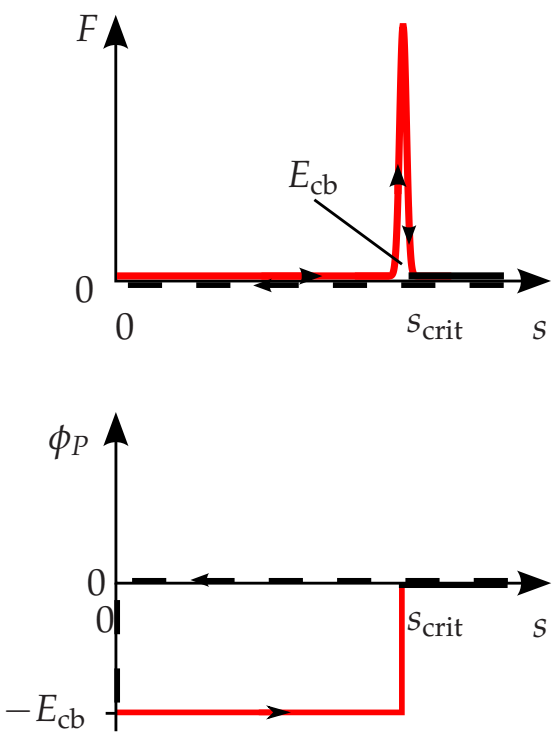

Fig. 2.5 The capillary bridge force $F$ as a function of the surface separation between two particles $s$ for the (a) minimal capillary model and (b) thin-thread model. Below each graph we find the pseudopotential of each force. Although the capillary bridge force is always a dissipative force field and, therefore, especially not conservative, it helps to imagine the acting forces by looking at a pseudo-potential, $\phi_{p}$. The formation of the bridge is hysteretic in both cases (minimal capillary model as well as thinthread model) which means that a collision $(s=0)$ has to occur in order to form a capillary bridge. The existence of the bridge is indicated by red. Approaching particles are visualized as dashed lines while solid lines denote retracting particles. In the minimal capillary model a constant bridge force, $F_{B}$, independent of $s$ is assumed and the capillary bridge energy is given by $E_{\mathrm{cb}}=F_{B} s_{\text {crit. }}$. In the thin-thread model a delta distributed force $F=\delta\left(s-s_{\text {crit }}\right)$ acts only at $s_{\text {crit }}$. The area under the distribution is the bridge energy $E_{\mathrm{cb}}$. For better comparison with the minimal capillary model an average capillary bridge force is defined as $F_{B}=E_{\mathrm{cb}} / s_{\text {crit }}$. In the pseudo-potential representation the thin-thread model looks like a square well potential. 
are caused by strong wind), as well as by horizontal or vertical vibrations which occur during earthquakes or by exertion of shear forces causing landslides. Other possibilities are the rotation of a mill, which is often used in industrial applications or, in numerical simulations, by a white noise stochastic thermostat. In the following the driving mechanisms, which are used in the present work, are introduced.

\subsubsection{Free cooling}

Free cooling is a driving technique which is mainly used in simulations or theoretical approaches (Brilliantov and Pöschel, 2004). Due to the absence of gravitational forces, which is assumed in this method, it is not easy to perform this driving method in experiments. Nevertheless, experiments for the free cooling of a quasi two-dimensional granular gas have been conducted (Maaß et al., 2008).

Free cooling is different from most other driving mechanisms because the driving is not provided constantly over time, but is reflected only in the initial conditions of the system. This usually means that the particle motion at the beginning is random and then slows down over time through dissipation in the system. This situation is well studied in the literature for dry granular matter (Goldhirsch and Zanetti, 1993; Brilliantov and Pöschel, 2004) and a first study for wet granular matter in one dimension is also available (Zaburdaev et al., 2006; Fingerle and Herminghaus, 2006). This driving mechanism is used to study the structures which emerge from a three-dimensional simulation of wet spheres and analyze the structures by means of the fractal dimension. The structures that emerge out of the three-dimensional simulation of wet grains can be seen in Fig.2.6. The results can be found in detail in Ulrich, Aspelmeier, Roeller, Fingerle, Herminghaus, and Zippelius (2009a) and Ulrich, Aspelmeier, Zippelius, Roeller, Fingerle, and Herminghaus (2009b).

\subsubsection{Random force}

The simplest form of driving (especially interesting in analytical calculations solving the Boltzmann equation) is a random force which is a stochastic thermostat using white noise ${ }^{5}$ (see e.g. Visco et al., 2005). This means that the different stochastic forces, $\chi$, acting on the particles are temporally and spatially uncorrelated and can be written

$$
\left\langle\chi_{i}^{\alpha}(t) \chi_{j}^{\beta}\left(t^{\prime}\right)\right\rangle=2 \gamma_{s} \delta_{\alpha \beta} \delta_{i j} \delta\left(t-t^{\prime}\right),
$$

where angular brackets denote the average over the noise source, $\gamma_{s}$ is the strength of the driving, $i, j$ are different particles, $\alpha, \beta$ are cartesian components of the $d$ dimensional space, $t, t^{\prime}$ are two points in time and $\delta$ with an index is the Kronecker delta, whereas the function $\delta\left(t-t^{\prime}\right)$ is the Dirac delta function.

5. This is used in a calculation in Appendix A 


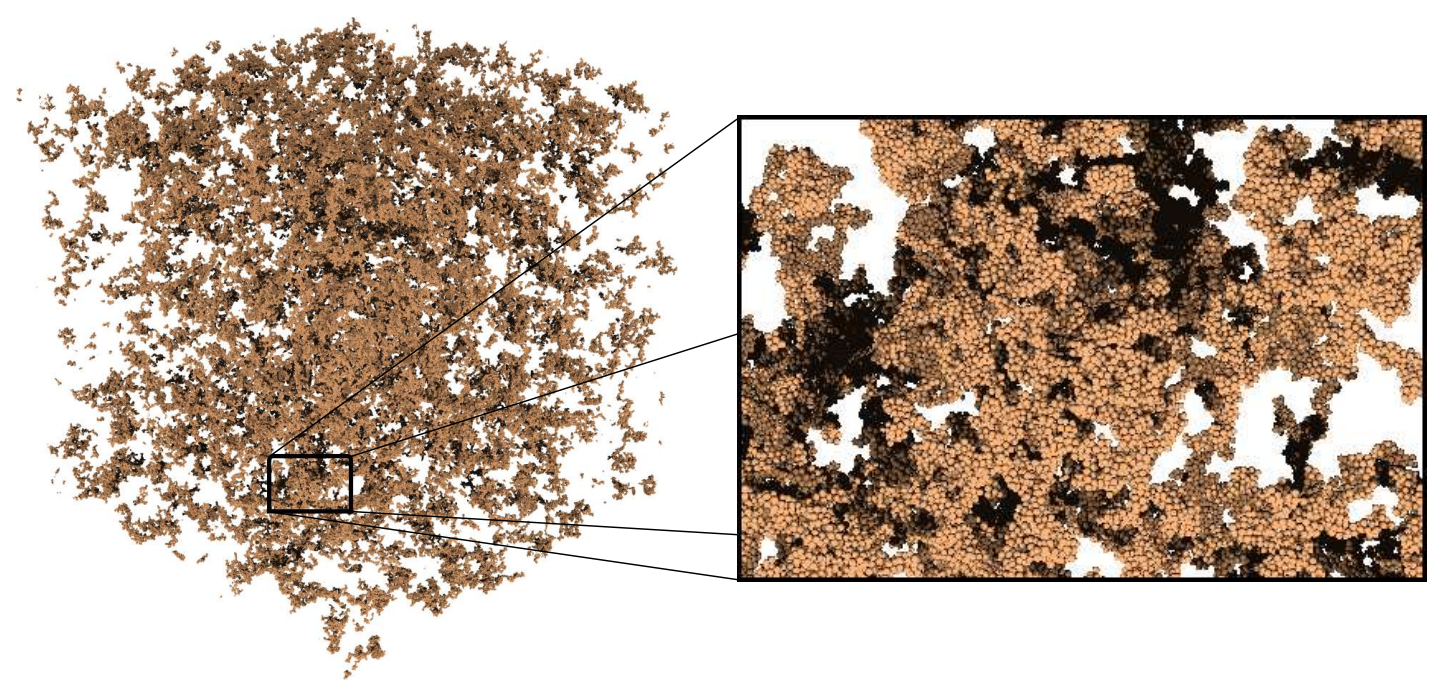

Fig. 2.6 Structures which evolve out of simulations of more than $2.5 \times 10^{6}$ spherical particles. The collisions between particles are elastic, but the capillary bridge interaction using the thin-thread model with an event-driven algorithm is dissipative. The particles are contained in a cubic box where each edge has length $L_{\text {box }} / d=600$, where $d$ is the diameter of the particles.

\subsubsection{Shaking}

Another driving mechanism to excite granular media is by vertical or horizontal vibration. This mechanism is present in nature, e.g. during earthquakes, and is also used in industrial applications to optimize the transport of granular matter by fluidization (Warr et al., 1995; Duran, 2006). In experimental studies this is a favorable driving mechanism because it is relatively easy to use. The setup is placed on a reasonably large loudspeaker and can then be vibrated with different driving functions using a function generator. The most common driving function in experiments is a sine with an amplitude $A$ and angular frequency $\omega$. The one-dimensional timedependent position of the container wall then reads

$$
f_{d}(t)=A \sin (\omega t)
$$

Even though it is possible to use other functions like a triangular motion or even white noise as a driving function, the simulations in this work are designed to be easily comparable with experiments and therefore a sinusoidal driving function is used. In many theoretical and numerical works about granular material, approximations of the sinusoidal motion by polynomials of first or second order (McNamara and Luding, 1998) can be found. The simplest form of shaking in event-driven molecular dynamics simulations is a sawtooth like motion in the limit of infinite frequency and zero amplitude, because the wall is stationary and only provides a certain energy input upon each collision of a particle with the wall. Because it is still an open question how the exact form of the driving function influences the dynam- 
ics of the system, sinusoidal shaking is used in this work in order not to lose the comparability with experiments.

Sinusoidal shaking is a straightforward method in molecular dynamics simulations (both time- and event-driven), however, in the context of this work, a method was developed to improve the performance of the standard approach in the case of the event-driven algorithm by more than one order of magnitude. A detailed description of this method can be found in Appendix B.

To characterize the strength of a sinusoidal driving function, the amplitude and oscillation frequency can be used. In this work, however, two different variables are used. The first parameter is the maximum kinetic energy, $E_{w}$, which a particle can obtain from a single collision with the wall (which is the parameter $S$ in Eshuis et al. (2005)) and is defined as follows:

$$
E_{w}=\frac{1}{2} m v_{w}^{2}
$$

with the mass, $m$, of the particle and the maximum velocity of the wall, for an arbitrary driving function $f_{d}(t)$,

$$
v_{w}=\max \left(\frac{\mathrm{d} f_{d}(t)}{\mathrm{d} t}\right) .
$$

In the case of wet granular matter Eq. (2.14) is used in a non-dimensionalized form

$$
E^{*}=\frac{E_{w}}{E_{\mathrm{cb}}} .
$$

The second paramter is the maximum acceleration which is normalized with respect to the acceleration due to gravity $g$

$$
\Gamma=\frac{\max \left(\frac{\mathrm{d}^{2} f_{d}(t)}{\mathrm{d} t^{2}}\right)}{g} .
$$

For the sinusoidal driving function of Eq. (2.13)

$$
\begin{aligned}
E^{*} & =\frac{\frac{1}{2} m(A \omega)^{2}}{E_{\mathrm{cb}}} \\
\Gamma & =\frac{A \omega^{2}}{g}
\end{aligned}
$$

is obtained. Sinusoidal shaking is used in chapters 3 to 6 and thus is the dominant driving mechanism used in this thesis. The period of the sinusoidal driving with angular frequency $\omega$ is given by $T_{\text {driv }}=2 \pi / \omega$. 


\subsubsection{Shearing}

The second driving mechanism presented here, which is used in chapters 7 and 8 is shearing. Even though both driving mechanisms (sinusoidal shaking as well as sinusoidal shearing) exert a sinusoidally varying force to the system, the main difference is that for shaking the driving force is uniform within the system but is changing sinusoidally with time and requires the existence of a physical boundary, for example a flat, elastically reflecting wall as used in this thesis. For shearing it is the exact opposite: the shear force is uniform in time but is changing sinusoidally with the spatial position within the system. Furthermore, the driving force is directly applied in the volume of the system and therefore does not require a physical boundary. Shear forces can be found wherever two surfaces are in motion relative to each other and are therefore present in many natural as well as industrial systems. Studying shear forces in granular systems experimentally is done by constructing two counter-rotating walls, which are covered by one layer of particles (see for example, Liao and Hsiau, 2010, and references therein). In simulations, shear forces can be applied to the system through a space-dependent external force field, $F(x)$, acting individually on each particle. Sometimes it is favorable to use periodic boundary conditions in simulations. Therefore, the width of the system is doubled, where the second half of the system is a mirrored copy of the initial system. As a linear shear profile would be non-differentiable at the transition from positive to negative slope, a cosine-shaped profile is used in this work which reads

$$
F_{S}(x)=F_{\text {smax }} \cos \left(2 \pi x / L_{x}\right)
$$

and can be seen in Fig.2.7 (Hoover, 1983; Schulz et al., 2003; Schulz and Schulz, 2006; Rahbari et al., 2009). The magnitude of the force varies along the $x$-direction in the system of width $L_{x}$, where $F_{\text {smax }}$ is the maximal possible force. The forces, however, do not change along the other lateral direction $y$, where the system is of length $L_{y}$. In order to be able to perfom event-driven simulations, the spacedependent cosine-shaped shear force is discretized such that the same force is acting in a certain region $\Delta x$, which is approximately of the order of the particle diameter ${ }^{6}$. In the three-dimensional system a gravitational force is also present, which leads to a sedimentation of the granular particles at the bottom and resembles a density close to random close packing. The mean kinetic energy of the system parallel, $E_{\mid 1}$, and perpendicular, $E_{\perp}$, to the driving is used as an order parameter in this system, indicated by the orange arrows in Fig. 2.7.

6. The calculation of the next collision time leads to a quadratic equation if the particles experience the same shear force. If the particles, however, experience different shear forces it involves a quartic equation which has to be solved. To solve this resulting quartic equations, the quartic solver by Don Herbison-Evans is used (Herbison-Evans, 1995) as it shows good numerical accuracy. 


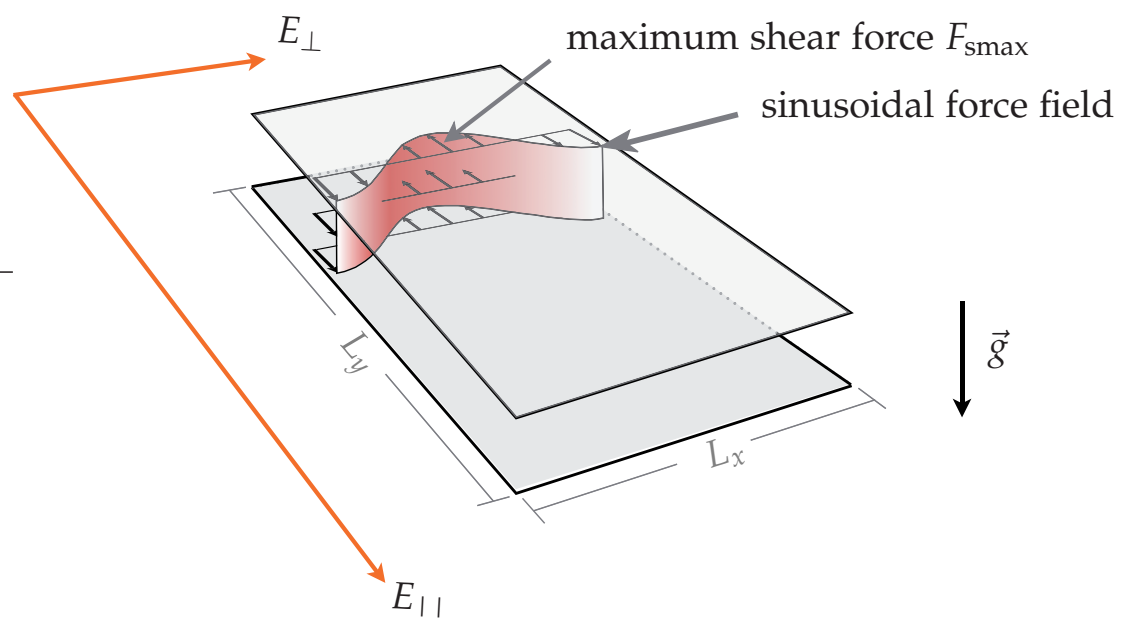

Fig. 2.7 Sketch of the flat system of width $L_{x}$ and length $L_{y}$ with periodic boundary condition in the two lateral directions and a elastically reflecting top and bottom plate. The space-dependent cosine-shaped force field is shown at the red plane indicated by the arrows. One position, where the maximum shear force, $F_{\text {smax }}$, is acting is marked. Additionally, a gravitational force $\vec{g}$ is acting downwards. The kinetic energy parallel, $E_{\|}$, and perpendicular, $E_{\perp}$, to the driving shear force serve as the order parameters (orange arrows).

\subsection{Particle size distribution}

One way of avoiding crystallization in a system of hard spheres (see e. g. Santen and Krauth, 2001; Laird and Davidchack, 2005) is to use slightly polydisperse spheres, i. e., spheres of different diameters. In this work, a uniform distribution of particle diameters is used as in Santen and Krauth (2001). The probability density function reads

$$
p_{\mathrm{df}}(d)=\left\{\begin{array}{rll}
\frac{1}{d_{\max }-d_{\min }} & \text { for } & d_{\min } \leqslant d \leqslant d_{\max } \\
0 & & \text { otherwise }
\end{array}\right.
$$

where $d$ is the actual particle diameter and $d_{\min }\left(d_{\max }\right)$ is the minimal (maximal) particle diameter of the sample. The variance of the uniform distribution are given by $\sigma_{d}^{2}=\left(d_{\max }-d_{\min }\right)^{2} / 12$.

Polydispersity, $\sigma_{p}$, is defined in this work as the normalized width of the distribution and reads for the uniform distribution

$$
\sigma_{p}=\frac{\sqrt{\sigma_{d}^{2}}}{\bar{d}}=\frac{d_{\max }-d_{\min }}{2 \sqrt{3} \bar{d}}
$$

where $\sigma_{d}^{2}$ is the variance of the particle size distribution of Eq. (2.21) and $\bar{d}$ is the average particle diameter. Choosing a certain polydispersity, $\sigma_{p}$, the maximum and minimum diameter is given by $d_{\max / \min }=\bar{d}\left(1 \pm \sqrt{3} \sigma_{p}\right)$. It is noted that the particle 
diameter is a positive number such that from $d_{\min } \geqslant 0$ it follows that the polydispersity is limited to values $\sigma_{p} \leqslant 1 / \sqrt{3}$.

Table 2.1 shows the particle size distributions, which were used in the experiments shown in this thesis. In Fig. 2.8 the probability density functions of the two different particle size distributions used in simulations is shown. The polydispersity in simulations is $\sigma_{p}=0.06$ (black) and $\sigma_{p}=0.02$ (red), which is close to the experimental values. Unless stated otherwise the polydispersity is $\sigma_{p}=0.06$ in simulations.

Table 2.1 Properties of the particles (Whitehouse scientific) used in the experiments shown in this thesis. The polydispersity is approximated as it contains the uncertainty of the sieving process.

\begin{tabular}{cccc}
\hline catalogue number & sieve fraction & mean diameter $\bar{d}$ & polydispersity $\sigma_{p}$ \\
\hline GP1060 & $1.00-1.12 \mathrm{~mm}$ & $1.06 \mathrm{~mm}$ & 0.03 \\
GP1215 & $1.18-1.25 \mathrm{~mm}$ & $1.22 \mathrm{~mm}$ & 0.02 \\
GP1500 & $1.40-1.60 \mathrm{~mm}$ & $1.50 \mathrm{~mm}$ & 0.05 \\
\hline
\end{tabular}

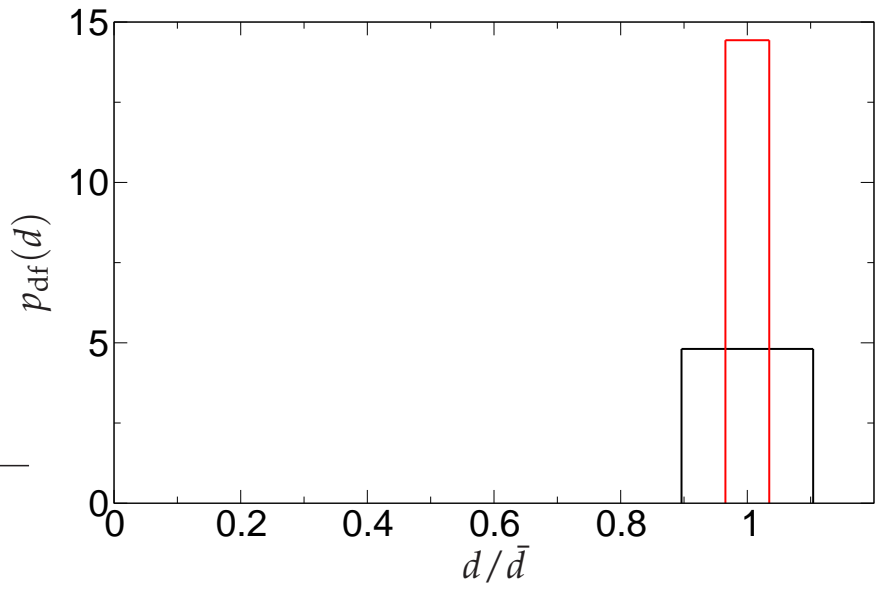

Fig. 2.8 Probability density functions of the two polydisperse particle size distributions, which are used in the simulations of this thesis. The polydispersity of the two samples is $\sigma_{p}=0.06$ (black) and $\sigma_{p}=0.02$ (red). 


\section{Phase diagrams of wet granular matter - sinusoidal shaking}

Phase transitions in condensed matter under thermally equilibrated conditions are by far the best understood examples of collective behavior and the probably most prominent example is the Ising model (Ising, 1924; Onsager, 1944). The astonishing fact that a solid melts at a precisely defined temperature, although the thermal energy is broadly distributed among the many degrees of freedom, can be completely apprehended from the principle of free energy minimization. Furthermore, the role of thermal fluctuations, which lead to the striking universality near critical points, was cast into a closed theory by means of the renormalization group (Wilson and Kogut, 1974). Hence, the physics of phase transitions at thermal equilibrium has matured into solid textbook knowledge (see, for instance, Yeomans, 1992).

Phase transitions far from equilibrium, on the contrary, are still far from being understood on such a general basis, despite their ubiquity and striking similarity. Well-known examples that are currently of great interest range from collective pattern formation in systems of molecular (Imbihl and Ertl, 1995) and micron scale (Fox et al., 2002; Chakrabarti et al., 2004) to transitions in social behavior (Farkas, 2002; Vollmer et al., 2006; Sumpter, 2006), besides granular matter. Many of the peculiar features of granular matter can be traced down to the dissipative nature of its grain scale dynamics. This gives rise to a wide variety of complex collective phenomena, and identifies granular matter as an interesting candidate for the investigation of physics far from thermal equilibrium. It has been proved useful to describe the collective behavior of a large number of grains with notions borrowed from the physics of equilibrium phase transitions. For instance, granular matter can behave like a solid, a liquid, or a gas (Jaeger et al., 1996; Duran, 2000), depending on the balance between energy injection and dissipation. As a particularly well controlled way of injecting energy, vertical vibration has been widely employed and explored in detail (Melo et al., 1995; Losert et al., 1999b; Umbanhowar et al., 1996; Wildman and Parker, 2002; Prevost et al., 2004; Götzendorfer et al., 2005; Götzendorfer et al., 2006a; Aranson and Tsimring, 2006; Huang et al., 2006; Reis et al., 2006; Clerc et al., 2008; Reyes and Urbach, 2008; Pacheco-Vázquez et al., 2009; Luding, 2009). While 'classical' research on granular systems tried to avoid any side effects due to cohesion, the dynamical behavior of wet granular matter has been attracting more and more inter- 
est of physicists recently (Bocquet et al., 2002; Herminghaus, 2005; Mitarai and Nori, 2006). The energy dissipation mechanism in wet granular matter is dramatically different from that of dry granular matter. In wet granular matter, the formation of capillary bridges between adjacent particles (Scheel et al., 2004, 2008) gives rise to a well defined energy scale in the system, which is absent in dry granulates (Herminghaus, 2005). Since the interest of physicists has only recently turned to wet granular matter, its dynamical behavior is still much less well understood than that of its dry counterpart (Herminghaus, 2005; Mitarai and Nori, 2006).

In the present chapter, the dynamics of wet granular matter under vertical agitation in a closed container is analyzed by means of numerical simulations. Aside from the solid-fluid transition, which is well known from dry granular systems, a second transition is presented which leads to the formation of a gas phase. While the solid-fluid transition is determined by the force acting on the sample as in the dry systems, the fluid-gas transition was found to be driven by the scale of the injected energy. Firstly, the phase diagram is presented using elastic collisions and it is demonstrated that many of its features are quantitatively independent of the details of the 'microscopic' interaction mediated by the capillary bridges. This may be viewed as some kind of universality in its own right. Secondly, the question is addressed how the phase transitions are affected by inelastic effects in the impact between grains and how the various transition lines scale with system parameters. It is tried to distinguish what properties can be seen as 'universal' (depending only on intrinsic parameters) and which have to be considered as non-universal (i.e., dependent on extrinsic parameters, like the volume of the container). Additionally, these findings are compared to experiments.

\subsection{Elastic collisions}

\subsubsection{Numerical setup}

To study the phase diagram of wet granular matter, molecular dynamics type simulations are performed in two and three dimensions with ideally circular or spherical, frictionless disks or spheres, each with mass $m=1$. The average particle diameter, $d$, is chosen to be $d=1.0$, while the polydispersity was $\sigma_{p}=0.06$ (cf.Sec. 2.5). In order to match the conditions of most experiments done on this topic (petri dish), the particles are confined in a flat container using periodic boundary conditions in the lateral directions, while the top and bottom elastically reflect particles. The attractive pairwise hysteretic interaction for capillary bridges in time-driven simulations was modeled with the minimal capillary model and in event-driven simulations with the thin-thread model (see Sec.2.3). The repulsion between particles is modeled by a Hertz-potential in the time-driven simulation and a hard-core repulsion in the event-driven simulation. A gravitational force with acceleration due to gravity, $g=1$, acting in the vertical direction is included in the simulations as well. The liquid content per grain is assumed to be the same everywhere and furthermore 
instantaneous bridge formation is assumed as the particle surfaces touch. Energy is injected into the container by vertical shaking with a sinusoidal driving function. At the beginning of each simulation the particles are equally distributed within the container on a regular lattice and heated to a granular temperature of $T_{g} / E_{\mathrm{cb}}=20.0$, which corresponds to a gas state.

\subsubsection{Stationary states}

Since a two-dimensional system with 1200 particles is simulated, different stationary states are found for different driving parameters of the sinusoidal shaking. States of lateral homogeneous areal-density appear in this system, which are considered to be solid- (s), fluid- (f) and gas-like (g) as shown in Fig.3.1. Aside from these homogeneous states, all three possible combinations of two coexisting phases can be seen. This is a solid-fluid (sfc), solid-gas (sgc) and fluid-gas coexistence (fgc) and can also be seen in Fig.3.1. To detect the state in which the system resides, order parameters, which are defined and explained in appendix 3.A to this chapter, are used.

In the main panel of Fig. 3.2, the areal density as well as the granular temperature, $T_{g}=\left\langle E_{\text {kin }}\right\rangle$, ('Boltzmann constant' $k_{B}=1$ and number of degrees of freedom $f_{\mathrm{df}}=2$ ) in units of the rupture energy, $E_{\mathrm{cb}}$, for a fluid-gas coexistence are plotted as a function of the lateral coordinate, on the same scale as the simulation box above. The granular temperature is found to vary over two orders of magnitude. This clearly demonstrates that the observed phenomenon is by no means akin to coexistence between thermodynamic phases, but is an intrinsically non-equilibrium state. The appearance of a cold dense phase in coexistence with a dilute hot phase complies qualitatively with earlier results obtained with magnetically cohesive granular beads (Blair and Kudrolli, 2003) or a thin vibrated granular layer of dry granular spheres(Prevost et al., 2004; Götzendorfer et al., 2005; Pacheco-Vázquez et al., 2009). Although that system does not exhibit a defined energy loss scale, as opposed to the system studied here, the essential feature is similar: in the dense phase, where the mean free path is small, the collision frequency is high. This gives rise to strong dissipation, and hence to very effective cooling. The figure also shows a comparison of the time- and event-driven simulation technique for otherwise identical system and driving parameters (solid versus dashed line). Reasonable agreement can be identified considering the fact that the latter is faster by more than a factor of hundred. Deviations occur mainly where density is high; this is expected since at high density, the thin thread model strongly underestimates cohesive effects (zero contact force). It is noted that the different models also have some noticeable influence on the interfacial density profile (Fig. 3.2). 


\section{Homogeneous states}

solid

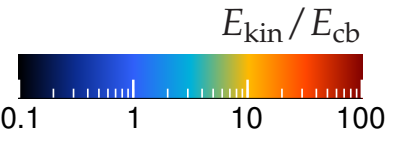

fluid

\section{Coexisting states}

solid - fluid

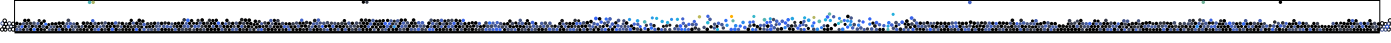

solid - gas

fluid - gas

Fig. 3.1 Two-dimensional time-driven molecular dynamics simulations of $1.2 \cdot 10^{3}$ wet granular spheres using the minimal capillary model. Vertical sinusoidal shaking is performed and periodic boundary conditions in the horizontal direction are used. Collisions between particles (and the wall) are assumed to be perfectly elastic. Three different homogeneous phases solid, fluid and gas are observed. Depending on the driving parameters, all possible combinations of states can be seen where two states coexist. The colors of the particles are calculated according to their individual kinetic energy normalized by the rupture energy. The particles are slightly polydisperse $\left(\sigma_{p}=0.06\right)$ with a mean diameter $d=1$. The system is $9 d \times 400 d$ particle diameters large, the rupture distance, normalized by the particle diameter, is $s_{\text {crit }} / d=0.0711$, the capillary bridge energy is $E_{\mathrm{cb}}=1.14$ and the mass of one particle is $m=1$. The values of the driving for the different states are as follows: solid: $\Gamma=30.0$ and $E^{*}=0.0022$, fluid: $\Gamma=30.0$ and $E^{*}=0.86$, gas: $\Gamma=30.0$ and $E^{*}=1.76$, solid - fluid: $\Gamma=1.6$ and $E^{*}=0.69$, solid gas: $\Gamma=1.15$ and $E^{*}=1.76$ and fluid - gas: $\Gamma=30.0$ and $E^{*}=1.27$. 


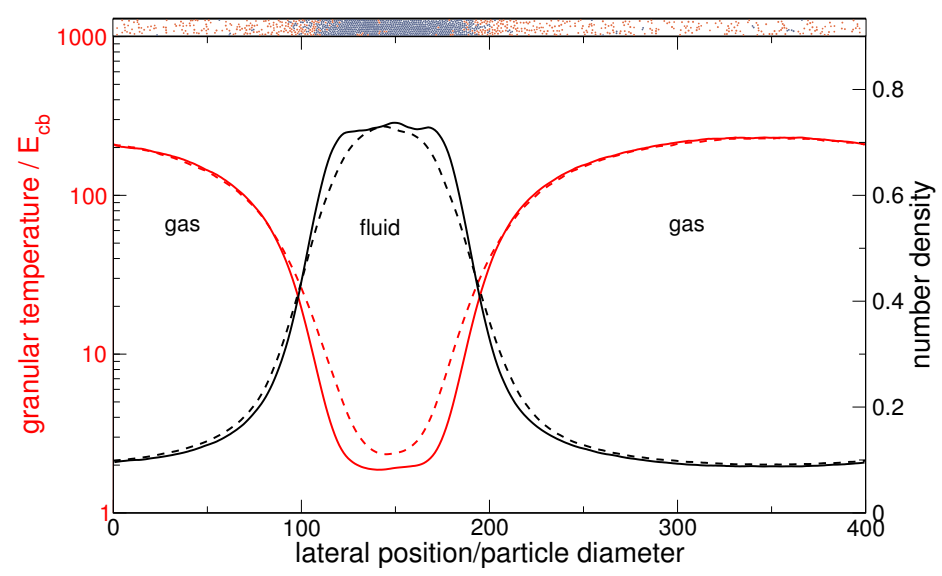

Fig. 3.2 Box on top: snapshot of molecular dynamics type simulation of 1200 particles at parameters where fluid-gas coexistence occurs. Blue grains have at least one capillary bridge, red grains have none. Main panel: Plot of the granular temperature (solid red curve) and the number density (solid black curve) obtained in time-driven MD simulations. The temperature varies laterally over two orders of magnitude, being high in the gas, but very low within the fluid plug. The curves are also displayed for event-driven simulations using the thin-thread model (dashed curves). Driving parameters are $\Gamma=15.0$ and $E^{*}=1.42$ other system parameters are similar as those used in Fig.3.1.

\subsubsection{Elastic phase diagrams}

The full phase diagram for a two-dimensional time-driven molecular dynamics simulation, which is obtained for variation of the amplitude $A$ and frequency $\omega$ of the external driving, is presented in Fig.3.3. It can be observed that for $\Gamma$ below unity, the system behaves as a solid for all frequencies. This happens because under these conditions, the gravitational force on the granular pile, as viewed in the rest frame of the system, is always pointing downwards, although sinusoidally varying in magnitude. As a consequence, the grains are never lifted from their support, and the position of each grain within the sample remains fixed as if there was no thermal motion induced by the driving (Duran, 2000). This represents a solid condensed state. As $\Gamma$ is increased above unity by increasing the amplitude, $A$, the pile periodically experiences a lifting force. For a dry granulate, this leads to fluidization at a certain threshold, $\Gamma_{c} \approx 1.2=: \Gamma_{\text {dry }}$ in experiments (Duran, 2000; Kim et al., 2002) showing a dependence on the driving frequency and the layer depth. The fluidization itself manifests by the onset of a mild movement of the grains, as it can be revealed by direct visual inspection. As liquid is added to the granulate (this corresponds, in the context of the minimal capillary model, to a non-zero attractive force with non-zero rupture separation), $\Gamma_{c}$ increases strongly. This corresponds to the dramatic difference in mechanical strength observed between a dry granulate, which flows almost like a liquid, and the visco-plastic texture of a wet granulate (Herminghaus, 2005). It was shown before that the increase in $\Gamma_{c}$ can be understood from the attractive interaction due to the liquid capillary bridges forming between adjacent spheres 
(Scheel et al., 2004; Herminghaus, 2005). In particular, the excess $\Gamma-\Gamma_{\text {dry }}$ must be merely large enough to overcome the forces exerted by these bridges by virtue of the surface tension of the liquid. The transition from the solid to the fluid phase is studied in more detail in chapter 4. Additionally, a transition from the fluid to the gas phase is observed, which, at sufficiently high $\Gamma$, also shows a coexistence region. The phase boundary is indicated by a dashed black line in Fig. 3.3. Above this line, a dense fluidized phase can coexist with a dilute gas-like phase. The latter shows up as a 'gas bubble'; an example was shown in Fig. 3.1. The term 'gas bubble' here and through this thesis refers to the volume of the system in which a granular gas is found.

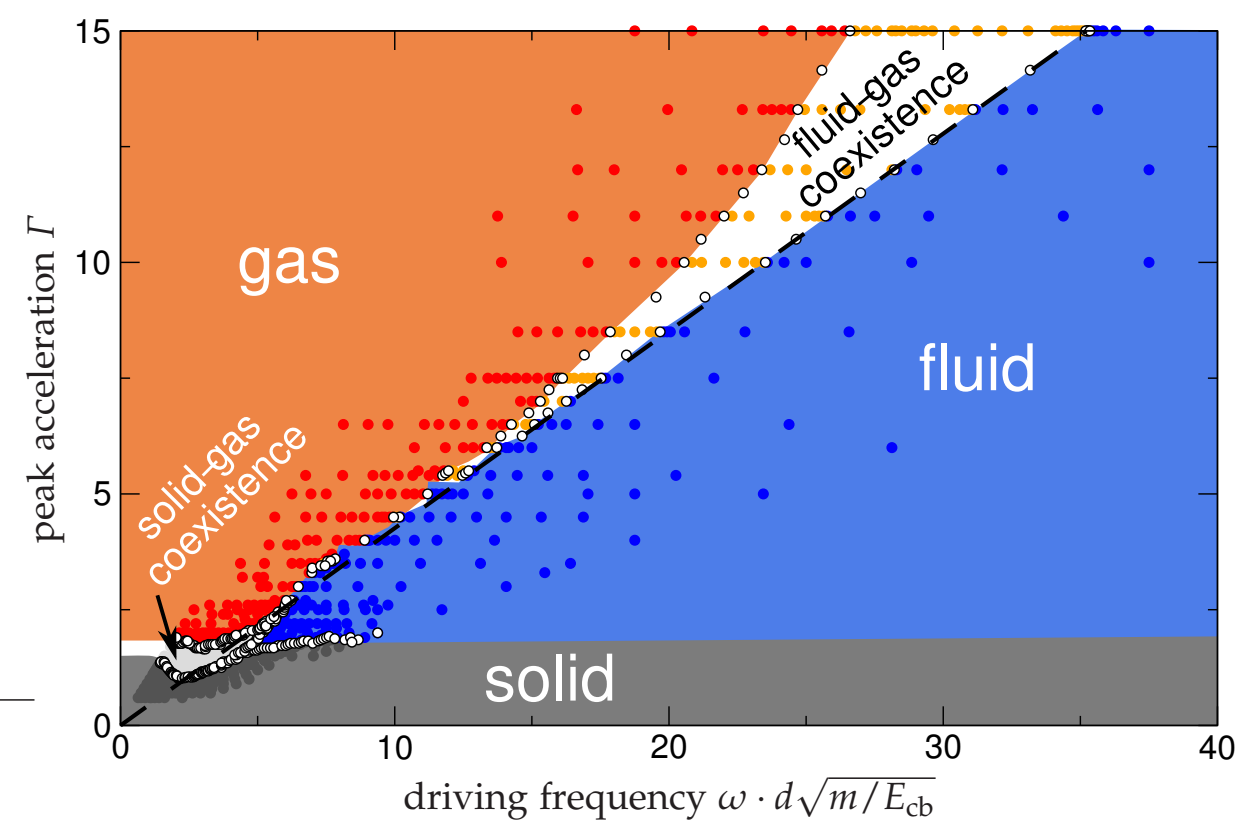

Fig. 3.3 Phase diagram of 1200 two-dimensional elastically colliding wet spheres using the timedriven molecular dynamics simulation shown in the plane spanned by the driving frequency, $\omega$, and the peak acceleration, $\Gamma$. The dashed line indicates the transition from fluid- to the gas-like state or the corresponding coexistence. The system parameters are the same as in the snapshots of Fig.3.1. To obtain the phase boundary in the two-dimensional phase diagram, a Delaunay triangulation of the simulated data points is performed and the mid-points between two points, which belong to different phases, is drawn as a white disk denoting the phase boundary. This can lead to a strange behavior that more than one transition point is marked for a constant value of e.g. $\Gamma$ if there is no data point at this respective value of $\Gamma$. Such points were removed by hand if this significantly improves the clarity of the presentation.

An obvious feature of Fig. $3 \cdot 3$ is that the data suggest the phase boundary between the fluid state and the fluid-gas coexistence to be a straight line meeting the origin of the diagram (dashed line). The quantity $\Gamma / \omega$, which is proportional to the peak velocity of the container walls, is constant along this line. This is interesting in regard to earlier experiments using a different agitation scheme (Geromichalos et al., 
2003). There, a pronounced transition, reminiscent of condensation from a gas into a viscous fluid, occurred when the peak velocity of the container walls came below a critical value, $v_{c}$. This value was found to correspond to the kinetic energy a bead must have in order to rupture the capillary bridge to a neighboring bead. It is given by $\frac{m}{2} v_{c}^{2}=E_{\mathrm{cb}}$, where $m$ is the mass of the bead and $E_{\mathrm{cb}} \propto d^{2} \gamma \sqrt{w}$ is the energy needed to rupture a capillary bridge (Herminghaus, 2005; Willett et al., 2000; Pitois, 2000; Pitois et al., 2001; Lian et al., 1998; Mikami et al., 1998; Simons and Fairbrother, 2000), $d$ is the mean diameter of the beads and $w$ denotes the liquid content of the sample, defined as the volume of the added liquid divided by the total volume of all glass spheres. Following this view, the dimensionless variable 'driving energy', $E^{*}=m v_{w}^{2} / 2 E_{\mathrm{cb}}$, was introduced in Sec. 2.4.3 as a control parameter. By varying the driving amplitude, $A$, and driving frequency, $\omega$, the two parameters $\Gamma$ and $E^{*}$ can be controlled independently.

It is enlightening to plot the observed transitions in the plane spanned by $\Gamma$ and $E^{*}$ on $\log$ /linear scale to better reveal the details. The result is shown in Fig.3.4(a). The transition from the solid (gray) to the fluid phase (blue) or, at higher $E^{*}$, from a solid-gas coexistence (white) to the gas phase (orange) is represented by a more or less horizontal line, indicating that this transition is independent of energy, but driven by acceleration $(\Gamma)$. In contrast, the fluid phase is separated from the fluidgas coexistence region (white) by a vertical line, indicating a transition driven by injected energy $\left(E^{*}\right)$, but independent of force. The fluid-gas coexistence region is bounded by a second line located at larger driving energy, which indicates the transition to a homogeneous gas-like state (orange). In this transition line, a bulge is observed where the excitation amplitude $A$ equals the rupture length $s_{\text {crit }}$ of the capillary bridges (solid line).

In order to investigate whether the observed behavior is of appreciable universality, the interaction potential used in the simulations was varied. In Fig.3.4(a), the circles represent simulations assuming a constant capillary force upon retraction (minimal capillary model), while the squares were obtained assuming a square-well retraction potential (thin-thread model, only the phase boundaries are shown as black squares with white edges). As the figure clearly shows, even these extreme cases give almost identical results. The only significant difference is the position of the solid to fluid phase boundary at small driving velocities, which is expected due to the vanishing contact force for the thin-thread model. The other details found in the phase transition lines do not seem to depend crucially on the interaction characteristics. The only relevant ingredient of the latter is that they are dissipative in the proposed hysteretic sense. A second phase diagram for larger capillary bridge energy is shown in Fig. 3.4(b). It is visible that the appearance of the bulge in the fluid-gas coexistence to gas phase boundary is indeed shifted along with the line, where the driving amplitude $A$ equals the rupture length $s_{\text {crit }}$.

The solid to fluid phase boundary in the phase diagrams of Fig.3.4 does not end in a critical point, which is impossible due to the symmetry breaking involved in this phase transition. 


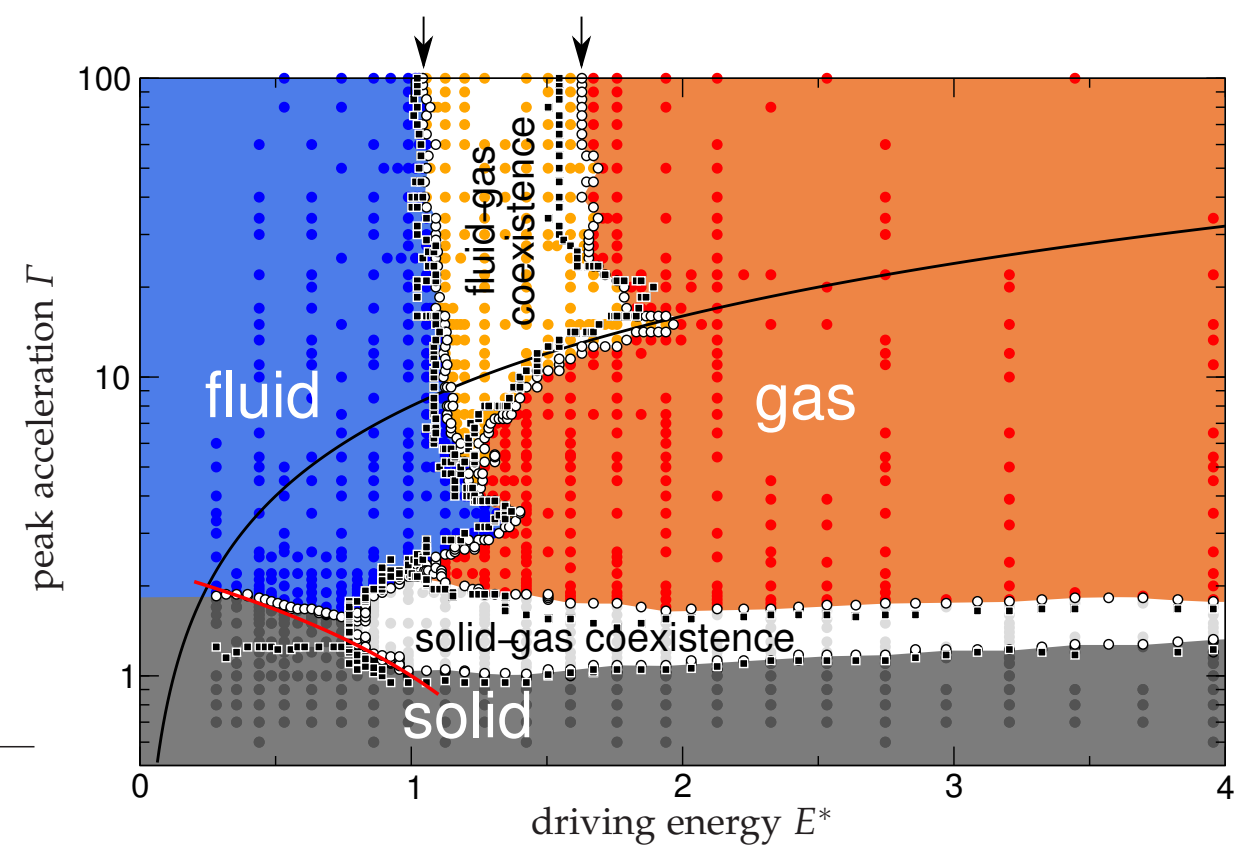

(a) $\tilde{E}_{\mathrm{cb}}:=E_{\mathrm{cb}} / F_{g} s_{\mathrm{crit}}=4.0$.

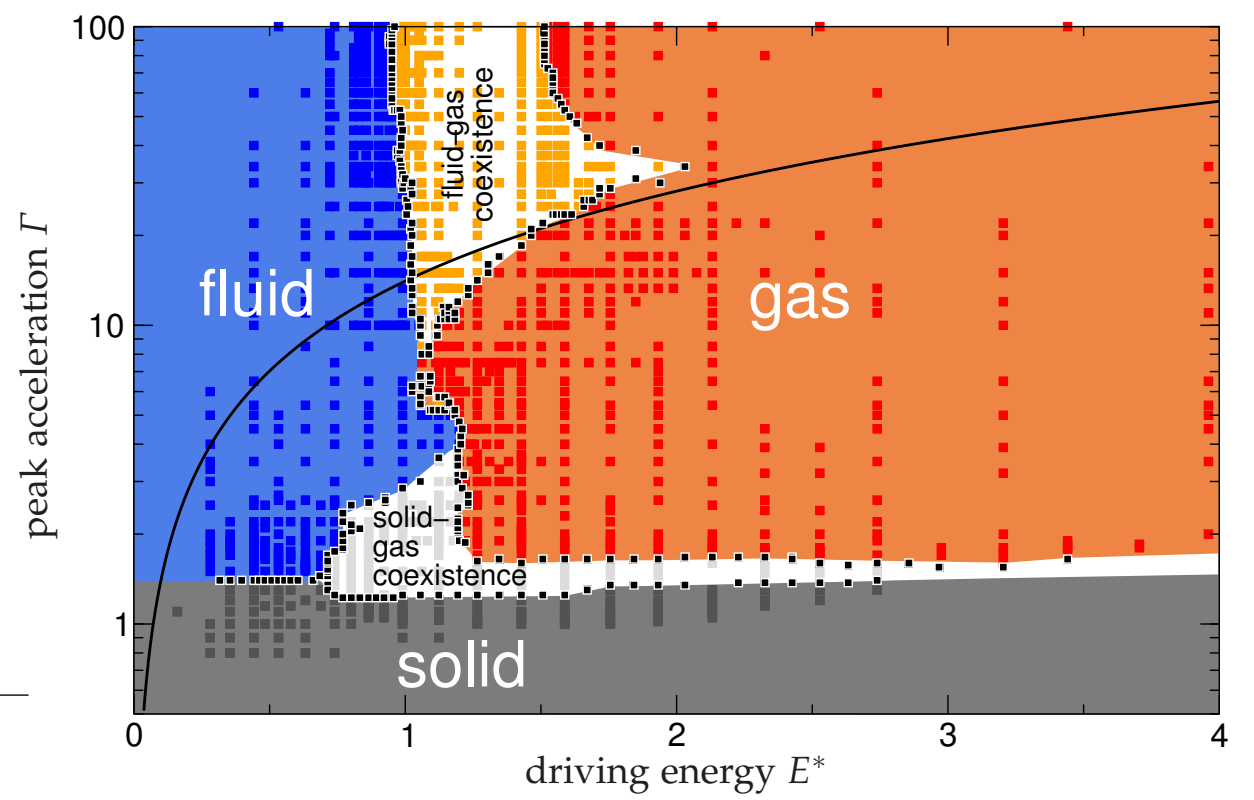

(b) $\tilde{E}_{\mathrm{cb}}:=E_{\mathrm{cb}} / F_{g} s_{\mathrm{crit}} \approx 7.0$.

Fig. 3.4 Two different phase diagrams from simulations with 1200 particles in two dimensions for different capillary bridge energies as denoted in the respective figure caption, where $F_{g}=m g$ is the graviational force acting on a particle. The horizontal boundary of the solid phase is clearly obtained, as well as the vertical fluid-gas boundaries. At the solid black curve the excitation amplitude $A$ equals the rupture length $s_{\text {crit }}$ of the capillary bridges. (a): The arrows at the top indicate the values of the respective phase transition. Circles denote time-driven simulations using the minimal capillary model while squares denote event-driven simulations using the thin-thread model. For the thin-thread model only the phase boundaries are shown (black squares with white edges). The solid red line is a theoretical prediction for the solid-fluid transition which is derived and explained in chapter 4. (b): Only the event-driven phase diagram using the thin-thread model is shown. 
In Fig. 3.5, the fluid-gas coexistence region is replotted in the plane spanned by the driving energy, $\frac{m}{2} v_{w}^{2}$, and the capillary bridge energy, $E_{\mathrm{cb}}$. They are rescaled as $\tilde{E}_{\text {kin }}:=m v_{w}^{2} / 2 F_{g} s_{\text {crit }}=v_{w}^{2} / 2 g s_{\text {crit }}$ and $\tilde{E}_{\mathrm{cb}}:=E_{\mathrm{cb}} / F_{g} s_{\text {crit }}$, respectively, where $F_{g}=m g$ is the graviational force acting on a particle. Obviously, $\tilde{E}_{\mathrm{kin}} \propto \tilde{E}_{\mathrm{cb}}$ for both transition lines, supporting that this transition is exclusively governed by the bridge rupture energy. The gravitational energy scale naturally separates out, in contrast to dry granulates (Götzendorfer et al., 2005).

Performing three-dimensional simulations ${ }^{1}$ shows that the influence of the dimensionality is significantly large. The transition line shifts from $E^{*}=1.02 \pm 0.02$ in two dimensions to $E^{*}=2.08 \pm 0.05$ in three dimensions, which is, within the measurement error, a shift by a factor of two. This is reasonable because the number of nearest neighbors is increasing from 6 in two dimensions to 12 in three dimensions for monodisperse spheres. This is at the same time the maximum number of capillary bridges which have to be broken to drive the particles into a gas phase. This supports the view that this transition is exclusively governed by the bridge rupture energy. It would be interesting to test if this scaling carries over to other dimensions than two or three.

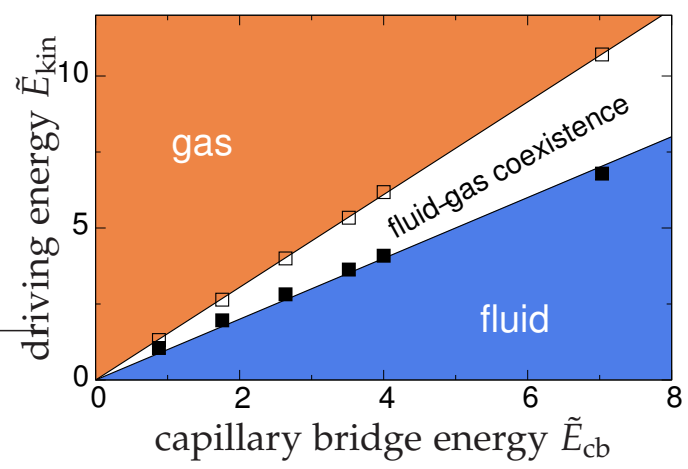

Fig. 3.5 Variation of the transition points with the surface tension, as obtained from simulations. The transition energies scale precisely with $E_{\mathrm{cb}}$. The slopes yield the critical (scaled) driving energies $E^{*}$ as $1.00 \pm 0.09$ and $1.52 \pm 0.02$, respectively (arrows in fig.3.4(a)) The quantities were rescaled as follows $\tilde{E}_{\text {kin }}=\frac{m}{2} v_{w}^{2} / F_{g} S_{\text {crit }}, \tilde{E}_{\mathrm{cb}}=E_{\mathrm{cb}} / F_{g} S_{\text {crit }}$.

\subsection{Theoretical description}

It is now tried to understand these findings in a more general framework. From the 'traditional' conditions invoked for the description of equilibrium phase transitions, only the homogeneity of the lateral pressure carries over to driven steady states, due to the required force balance at the phase boundaries. In contrast, the familiar uniformity of temperature breaks down. In what follows, the mechanism by which

1. System parameters: $1.2 \times 10^{5}$ particles, box size $8 d \times 192.5 d \times 192.5 d, s_{\text {crit }} / d=0.0711, E_{\mathrm{cb}}=1.14$. Not shown in a graph. 
the symmetry in the formerly homogeneous system is broken, and how the system finds its steady state within the broken symmetry is sought.

The power injected into the system from the oscillating walls, $P_{\text {inj, }}$ must be balanced at each lateral position within the sample by dissipation, $P_{\text {diss }}$. Assuming a certain velocity distribution for the grains, both quantities can be evaluated in a straightforward way, such that the net power $\Delta P\left(T_{g}\right)=P_{\text {diss }}-P_{\text {inj }}$ can be obtained as a function of temperature (Fingerle and Herminghaus, 2008). Clearly, in a dissipative system like the one examined, the velocity distribution will deviate from the 'usual' Gaussian (cf. Appendix A), exhibiting non-Gaussian 'tails' and broken equipartition of energy (Brey and Ruiz-Montero, 2009). However, these affect only a small fraction of the particles (Rouyer and Menon, 2000) and are of minor impact on the average collision frequency, which in turn determines the dissipation rate. In order to compute the above-mentioned quantities, it thus appears sufficient to use a Gaussian velocity distribution for the sake of simplicity.

Fig. 3.6 shows the obtained result, displayed for three values of the driving energy, $E^{*}$. For $E^{*}<0.67$, only one stable (i.e. positive slope) zero is obtained, which is at low temperature. It corresponds to a moderately dense state, which has just enough free volume for the critical separation $s_{\text {crit }}$ to be exceeded frequently enough to balance the injected power, $P_{\text {inj. }}$. On the other hand, for $E^{*}>2.22$, again only one stable zero exists, but this corresponds to a high temperature. It represents a dilute phase, whose temperature is determined mainly by the balance of the energy uptake from the motion of the boundaries with the dissipation due to the 'wet' impacts with them.

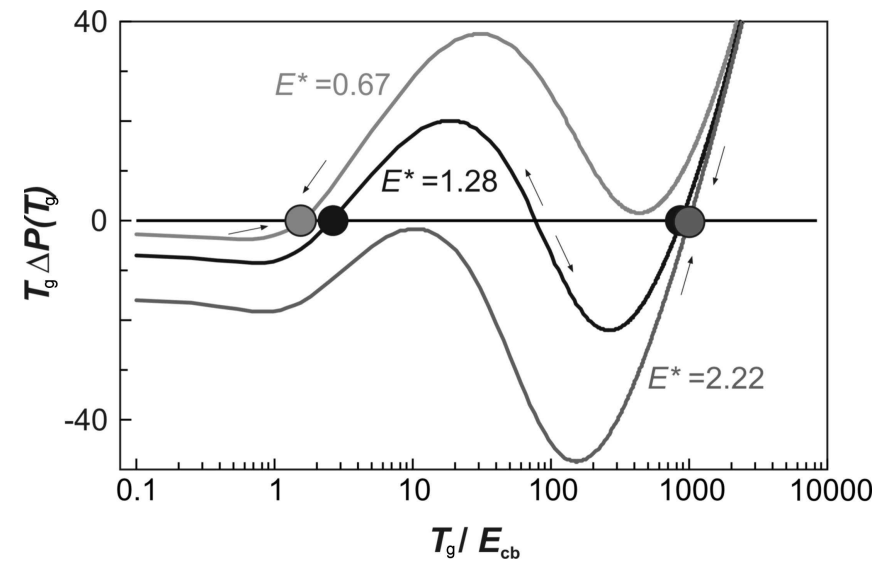

Fig. 3.6 The net power, $\Delta P=P_{\text {diss }}-P_{\text {inj }}$, as a function of the granular temperature. $\Delta P$ is multiplied by the granular temperature here in order to achieve a clear presentation of the data in a single graph. Depending on the driving energy, $E^{*}$, either one (gray) or two (black) stable zeros are obtained, corresponding to a single phase or two-phase coexistence, respectively. The arrows denote the response of the system to small fluctuations. Figure taken from (Fingerle et al., 2008).

At intermediate velocities (black curve in Fig.3.6), two stable zeros (black circles) are obtained, another for low and one for high temperature. This is to be identified 
with the fluid/gas coexistence observed in the simulations for intermediate velocities. Since this continuum theory does not take gravity into account, it is intrinsically independent of the parameter $\Gamma$ used in Fig. 3.4, and thus corresponds to the vertical lines in the phase diagram. The mean field model predicts the coexistence in the stripe $0.67<E^{*}<2.22$ of the phase diagram, which compares quite favorably with Fig. 3.5 for $\Gamma \gg 1$ (where gravity is irrelevant) given the crudeness of the involved assumptions.

\subsection{Inelastic collisions and comparison with experiments}

The assumption of elastic collisions between particles is a convenient way to perform simulations and get first insight into the behavior of wet granular matter, because it is possible to study the pure influence of the capillary bridges. However, elastic collisions between particles are not very close to the behavior of real granular matter which always undergoes inelastic collisions. Therefore, inelastic collisions ${ }^{2}$, as described in Sec.2.2, are incorporated in the simulation in order to obtain quantitative agreement between simulations and experiments. The numerical results in this section are obtained by event-driven molecular dynamics simulations using the thinthread model which showed almost perfect quantitative agreement in Fig. 3.4(a).

\subsubsection{Experimental setup}

Fig. 3.7 is a sketch of the experimental setup. Glass spheres with diameters $1.06 \mathrm{~mm}$, $1.22 \mathrm{~mm}$ and $1.5 \mathrm{~mm}$ (Whitehouse scientific, GP1060, GP1215, GP1500) are used as the granular sample (see Sec.2.5). The glass beads were cleaned subsequently with ethanol, acetone, and Millipore water, and dried in an oven before use. The glass beads were placed into a flat cylindrical glass container, with a glass lid mounted on a separate stand. The latter fits well enough into the container to prevent glass beads from exiting, but enables free exchange of air, such that the gas pressure inside the cell was kept at atmospheric pressure. The height of the glass bead fill, $h$, was well below the total height of the cell, $H$, which could be adjusted by vertically moving the glass lid. A certain amount of wetting liquid was mixed into the sample before the experiment. Water, which has a surface tension of $\gamma \approx 72 \mathrm{mN} / \mathrm{m}$, was mostly used to wet the sample. Silicone oil (AK 5 , Wacker; $\gamma \approx 21 \mathrm{mN} / \mathrm{m}$ ), was also used for comparison. Both liquids wet the glass surface well, with a contact angle below 10 degrees. The liquid content, $W$, is defined as the ratio between the volume of the added wetting liquid and the total volume the sample occupies (i. e., the area of the sample cell times the height of the granular pile, $h$ ). The container, which has a diameter of $145 \mathrm{~mm}$, was mounted on an electromagnetic shaker (Tira TV588o/LS). A sinusoidal signal from a function generator is used to drive the shaker. The strength

2. The collisions between a particle and the wall is for simplicity still assumed to be elastic. This is sometimes also used in literature (for example Grossman et al., 1997) 


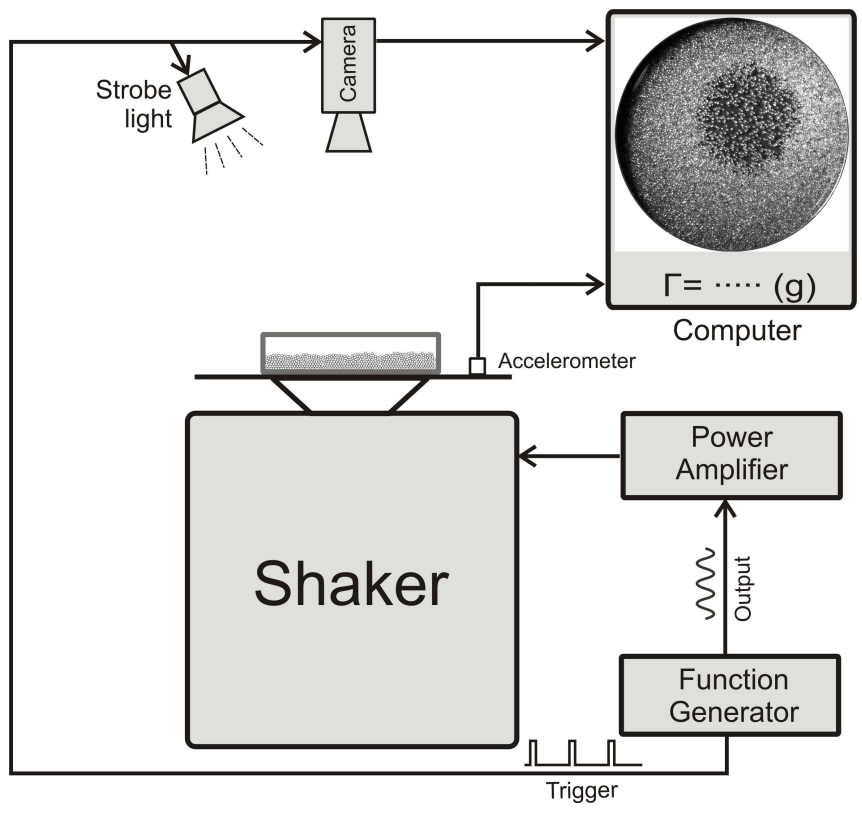

Fig. 3.7 Sketch of the experimental setup. Function generator, strobe light source and the high speed camera are synchronized with each other so that the images are captured at a certain phase of each vibration cycle. An example of a top view of the sample is shown in the inset photograph on the top right.

of the vibration is measured by an accelerometer (Kistler $8702 \mathrm{~B} 100 \mathrm{M} 1$ ). The sample is illuminated with a strobe light (Helio Strobe Beta A2) and viewed from top by a high speed camera (PCO 1200s). The camera is externally triggered by the function generator so that the image is captured at the same phase of each vibration cycle. All measurements are taken after the added wetting liquid has been distributed homogeneously within the sample by shaking the sample at a low frequency $f<20 \mathrm{~Hz}$ and high acceleration $\Gamma \approx 10$ for few minutes.

\subsubsection{Results and discussions}

\subsubsection{Observations in experiments}

As the frequency and amplitude of the drive are varied, a number of characteristic changes in the appearance of the sample are observed. At low acceleration, the glass beads follow the harmonic vertical motion of the container, but no site exchange processes take place. This can be assessed most conveniently by the strobe light illumination, which reveals a completely static sample in this case. As the acceleration is increased, however, relative movements of the beads are observed, or even the appearance of a 'bubble' of a particularly 'hot' granular gas, surrounded by a 'fluid' (i. e., dense but mobile) phase.

The phase diagrams are measured by keeping the driving frequency, $f$, at a certain 
value and varying the peak vibration acceleration, $\Gamma$. The transition from the solid (s) to the fluid (f) phase is determined experimentally by first completely fluidizing the sample, followed by slowly decreasing $\Gamma$ until the particles stop moving which is detected by direct visual inspection. This criterion turned out to be better defined than just increasing $\Gamma$ and detect the start of movement of particles: This is assumed to be due to the difficulty to initialize the system with reproducible packing geometry. The transition from the fluid phase to the fluid-gas coexistence (fgc) occurs at an acceleration where a 'gas bubble' (see the top view image embedded in Fig.3.7) nucleates. After nucleation, the (circular) granular 'gas bubble' grows spontaneously to a certain size. As $\Gamma$ is decreased again, the size of the gas bubble decreases continuously to zero, which indicates that there is a significant hysteresis for this transition. Within the hysteresis regime, the 'gas bubble' is unstable and fluctuates strongly in size. These fluctuations make a precise determination of the point of vanishing bubble size very difficult. Therefore, The f-fgc transition is rather defined as the acceleration at which nucleation is observed. It is clear that the fluctuations of the gas bubbles close to the phase boundary are worthy of further investigations. The transition from the fluid-gas coexistence $(\mathrm{fgc})$ to the gas $(\mathrm{g})$ phase again takes place at a well defined value of $\Gamma$, and leads to a completely homogeneous gaseous state of the sample. The abbreviations s, $\mathrm{f}, \mathrm{sfc}, \mathrm{sgc}, \mathrm{fgc}$ and $\mathrm{g}$ are henceforth used for solid, fluid, solid-fluid coexistence, solid-gas coexistence, fluid-gas coexistence, and gas phases, respectively.

\subsubsection{Phase diagram: experiments vs. simulations}

Fig. 3.8 shows the experimentally determined phase diagram in both $\Gamma$ - $f$ and $\Gamma-E^{*}$ planes. The s-f boundary is located at $\Gamma \approx 1.9$, only weakly dependent on the driving frequency, $f$. It therefore lies higher than the corresponding transition for dry granular matter ( $\Gamma \approx 1.2$ (Duran, 2000; Kim et al., 2002)). This increase represents the enhanced mechanical stability of the granulate due to the network of capillary bridges forming between the grains. The independence of the critical acceleration upon the frequency of the driving underscores its physical significance. In fact, since the acceleration is directly related to a force, this transition has been identified as mainly 'force driven', which is supported by this experiment. In the fluid-gas coexistence regime (fgc) (cf. photograph in Fig. 3.7), the size of the 'gas bubble' grows with the driving amplitude, while keeping its circular shape. During this growth, the surrounding fluid phase is pushed aside, and the height of the granular fluid increases until it reaches the top of the container. When the drive is increased further, the size of the 'gas bubble' saturates, and the density difference between the gas and fluid phase decreases until the sample finally enters a homogeneous gas phase. This corresponds to approaching the boundary between the coexistence regime ( $\mathrm{fgc}$ ) and the gaseous phase $(\mathrm{g})$.

As shown in Fig. 3.8(b), the f-fgc transition occurs at a certain driving energy, $E^{*}$. This strongly suggests that this transition is linked to a certain characteristic 


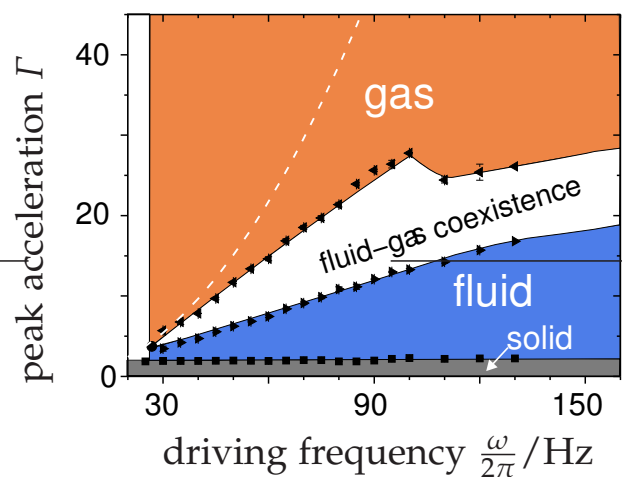

(a)

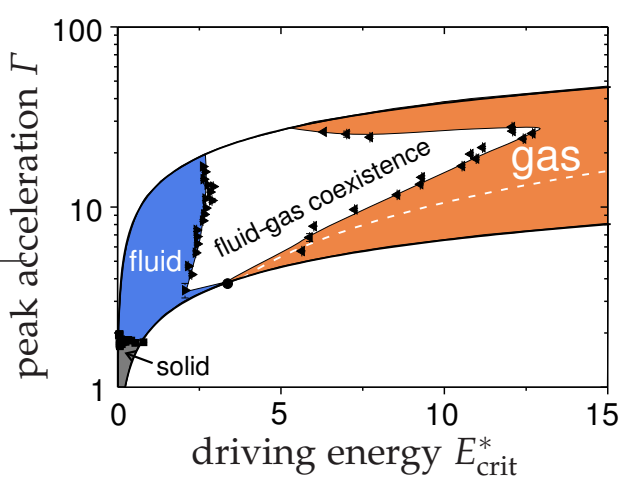

(b)

Fig. 3.8 Phase diagram of water wetting glass spheres with diameter $d=1.5 \mathrm{~mm}$ and liquid content $W=1 \%$ in driving acceleration $\Gamma$ - frequency $f$ plane (a) and $\Gamma$ - driving energy ( $\left.E^{*}\right)$ plane (b). Filling height of the sample is $55 \%$ of the container height $H=6.6 \mathrm{~mm}$. Regions color coded by gray, blue, white and orange correspond to solid (s), fluid (f), fluid-gas coexistence (fgc) and gas (g) phases. The white unlabeled regions in the current and following plots are unexplored due to the limitation of the apparatus. The solid black dot corresponds to the converging point of the f-fgc and fgc- $g$ transition lines. The white dashed line indicates $A=d=1.5 \mathrm{~mm}$, where $A$ is the vibration amplitude. The experiment was performed by K. Huang.

kinetic energy per particle-wall collision. As the simple mean-field model in Sec. 3.2 predicts, the (fgc)-(g) boundary should be just parallel to the $(\mathrm{f})-(\mathrm{fgc})$ boundary, at slightly higher energy. This is clearly not the case: the observed structure of the phase boundary is much more complex. Particularly prominent is the sharp backward bend which occurs at $\Gamma \approx 25 \mathrm{~g}=: \Gamma_{b}$.

A glance back at Fig. 3.8(a) shows that the f-fgc and fgc-g transition lines in the phase diagram approach each other as the driving frequency decreases (Fig. 3.8(a)). They seem to intersect at $f_{c} \approx 27 \mathrm{~Hz}$ and $\Gamma_{c} \approx 3.0$. This point can be accurately detected by varying both the driving frequency and acceleration. The driving frequency, $f$, is continuously decreased, while the size of the gas bubble is kept maximal by varying $\Gamma$, until the gas bubble vanishes. The data points shown in Fig. 3.8 are averages of four measurements each, and the error bar is within the size of the data points. In an attempt to rationalize this finding, it is noted that $f_{c}$ compares favorably with a natural time scale $t_{g}$ of the system, which can be derived from the attractive capillary force and the bead mass, $m$. Setting $t_{g}:=\sqrt{m / \gamma}$, we have $f_{g}=1 /\left(2 \pi t_{g}\right) \approx 45 \mathrm{~Hz}$, which is not far from $f_{c}$. This clearly must be investigated in further detail.

It was shown in Sec. 3.1 using elastic collisions that the f-fgc transition occurs at $E^{*}=1$, where $E^{*}$ is the kinetic energy injected by one collision with the wall normalized by the rupture energy $E_{\mathrm{cb}}$. In the experimental phase diagram shown in Fig. $3.8(\mathrm{~b})$, it can be observed that the fluid to fluid-gas coexistence (f-fgc) transition occurs at $E^{*} \approx 2.5$, which is significantly larger than unity, as obtained before in elas- 
tic simulations. This shift is interpreted as being due to the influence of inelasticity in the collisions, frictional forces and dimensionality.

To support this view, in Fig. 3.9 the phase diagram is presented for slightly inelastic collisions with a coefficient of restitution of $\varepsilon=0.96$. All other system parameters are identical to the one used in the phase diagram shown in Fig.3.4(a). Comparing these two phase diagrams (Fig. 3.4 vs. Fig. 3.9) shows that the two mechanisms for force and energy driven phase transitions, which appear as vertical and horizontal line, are similarly obtained, but the f-fgc transition indeed occurs at $E^{*} \approx 1.84 \pm 0.09$ well above $E^{*}=1.02 \pm 0.02$, which is in qualitative agreement with the experimental findings. Performing three-dimensional simulations ${ }^{3}$ shows that the transition line shifts from $E *=2.08 \pm 0.05$ in the elastic case to $E *=4.36 \pm 0.14$ in the inelastic case with a coefficient of restitution of $\varepsilon=0.96$. This shift is, within the error bars, almost by the same factor as in the two-dimensional case. The small remaining difference can be understood in terms of number of layers, which was 3.0 in the two-dimensional case and 3.24 in the three-dimensional case. This will be discussed in more detail in Sec.3.3.2.3.

The bulge which was observed in the phase boundary of the fluid-gas coexistence and gas phase (in the phase diagram using elastic collisions) vanishes completely and the phase boundary in the inelastic case is then given by a straight line. The most significant difference between the phase diagrams of elastic and inelastic particle interactions is that a third coexistence region appears which must therefore be caused by the inelasticity. In order to test if this new coexisting region is solely induced by the inelastic collisions or by some interplay between the capillary interaction and the inelasticity, a simulation of dry granular matter is performed with the same coefficient of restitution, $\varepsilon=0.96$, but no dissipation through capillary bridges, $E_{\mathrm{cb}}=0$. The resulting phase diagram of dry granular matter is shown in Fig.3.10 and indeed only this newly appeared coexistence region, which is present in the phase diagram of inelastic wet granular matter in Fig. 3.9, is visible. Furthermore, the solid black line in Fig. 3.10 indicates where the amplitude of the driving equals one particle diameter and it can be seen that the coexistence occurs only if the amplitude of the driving is close to a critical driving amplitude, $A_{\text {crit }}$. The dependence of $A_{\text {crit }}$ on the inelasticity, $\varepsilon$ is discussed in more detail in Sec.3.3.2.4. This seems to be a geometry-driven effect which is intimately connected to inelasticity. There were several coexistence phenomena reported in different settings of dry granular matter so far (Eggers, 1999; Javier Brey et al., 2001; Wildman and Parker, 2002; Prevost et al., 2004; Götzendorfer et al., 2005; Pacheco-Vázquez et al., 2009). However, in contrast to the system reported in Eggers (1999); Javier Brey et al. (2001); van der Weele et al. (2001), the coexistence in the present system appears completely without introducing a spatial inhomogeneity in the system and is stationary in contrast to Moon et al. (2001). Furthermore, the particles in the system studied in this chapter are polydis-

3. System parameters of the inelastic simulation: $1.2 \times 10^{5}$ particles, box size $9 d \times 192.5 d \times 192.5 d$, on average 3.24 layers, $s_{\text {crit }} / d=0.0711, E_{\mathrm{cb}}=1.14$. 


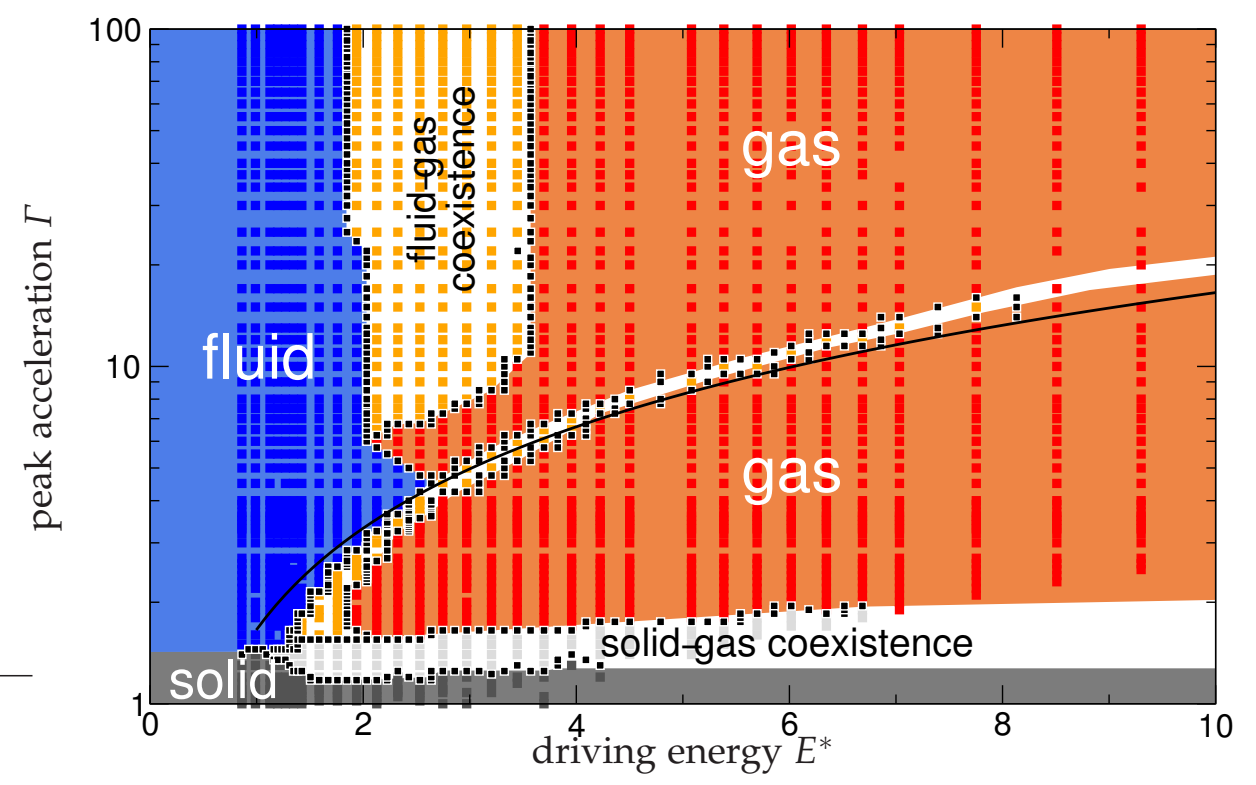

Fig. 3.9 Phase diagram of 1200 two-dimensional granular disks with a coefficient of restitution $\varepsilon=0.96$ for collisions between particles using the thin thread model. Other system parameters are identical to the ones used in the phase diagram of Fig.3.4(a). A third coexistence region is emerging by the incorporation of inelastic collisions, which appears at a constant driving amplitude (black line, with $A=$ $0.34 d$.

perse and are neither a binary mixture of particles nor monodisperse (Wildman and Parker, 2002; Pacheco-Vázquez et al., 2009) and the system is confined by a top lid such that the system is not open in contrast to the one used in Götzendorfer et al. (2005); Pacheco-Vázquez et al. (2009). Last but not least, the coexistence does not involve a solid phase or crystallized parts (Prevost et al., 2004; Götzendorfer et al., 2005; Pacheco-Vázquez et al., 2009). Therefore, it seems that the fluid-gas coexistence in dry granular matter observed in this thesis is a phenomenon not reported in literature so far. However, this needs further investigation for a concluding identification of the underlying mechanism.

As the inelasticity is increased to a coefficient of restitution of $\varepsilon=0.8$, the phase diagram is obtained, which is shown in Fig.3.11. It can be seen that both the usual fluid-gas coexistence region and the fluid-gas coexistence region caused by the inelasticity increase their size in the phase diagram and therefore emerge in a larger parameter range of the driving. Obviously, both coexistence regions start to merge, suggesting a superposition of both mechanisms. The solid black line in Fig. 3.11 indicates where the driving amplitude equals one particle diameter, which is in approximate quantitative agreement with the experiment shown in Fig. 3.8(b). Furthermore, the position of the horizontal s-f transition line agrees quantitatively with experiment. The solid-gas coexistence in this phase diagram is not presented because it is 


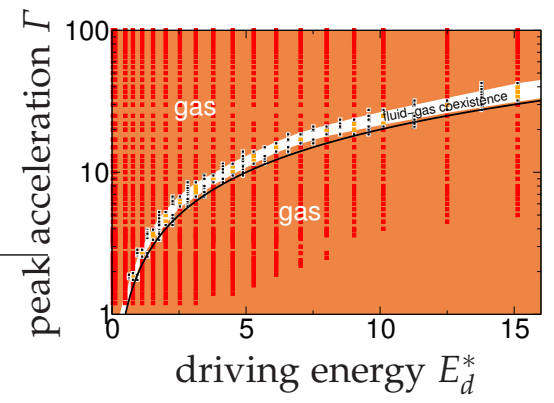

Fig. 3.10 Phase diagram of dry granular matter with $E_{\mathrm{cb}}=0$ and coefficient of restitution $\varepsilon=0.96$. Except for the height of the system, which is $18 \cdot d$, all other parameters are identical to the ones used for the phase diagram in Fig.3.9. The axis of the driving energy is normalized as $E_{d}^{*}=\frac{\frac{1}{2} m(A \omega)^{2}}{m g d}$, where $m$ is the mass of one particle, $g$ is the acceleration due to gravity and $d$ is the mean particle diameter. The solid black line denotes where the amplitude of the driving equals the mean diameter of the particles.

outside the range of simulated driving amplitudes ${ }^{4}$.

\subsubsection{Scaling of the f-fgc transition}

The variation of the positions of the transition lines, as different system parameters are varied, is now investigated, in order to check the ideas put forward above. Fig. 3.12 shows phase diagrams for different liquid content, $W$, and particle diameter, $d$. As the liquid content and the particle diameter change the rupture energy of capillary bridges, $E_{b} \propto R^{2} \gamma \sqrt{W}$, varies. By choosing the rescaled driving energy, $E^{*}$, as the abscissa, the data for the $\mathrm{f}$-fgc transition collapse on a single line within experimental scattering. This indicates that this transition is indeed dominated by the rupture energy alone.

Additionally, the surface tension, $\gamma$, of the wetting liquid was varied since it is connected to the capillary bridge force as $F_{B} \propto \pi \gamma d$. As shown in Fig. 3.13, phase diagrams for different wetting liquid are investigated for $d=1 \mathrm{~mm}$ particles. Again, approximate data collapse for the f-fgc transition is observed at high peak accelerations $\Gamma$. This confirms that the scaling with rupture energy of capillary bridges in this region is appropriate and viscosity effects can be neglected in first order approximation, which is in agreement with the results shown in Fig. 3.5 for elastic simulations.

The influence of system dimensions on the observed phase transitions by varying the sample height $(h)$ and the container height $(H)$, as shown in Fig.3.14, was also investigated. While the fgc-g transition line shifts substantially to the right as $H$ is increased while $h$ is kept constant, the $\mathrm{f}$-fgc transition line remains almost unchanged. In contrast, changing the sample height, $h$, at constant $H$ mainly affects the f-fgc transition, but not the fgc-g transition. It is concluded that, while the fgc-g transition is determined by container size and should therefore be considered 'non-universal',

4. The value of the driving amplitude was limited in order to be able to use certain optimization techniques to improve the speed of the simulation program. 


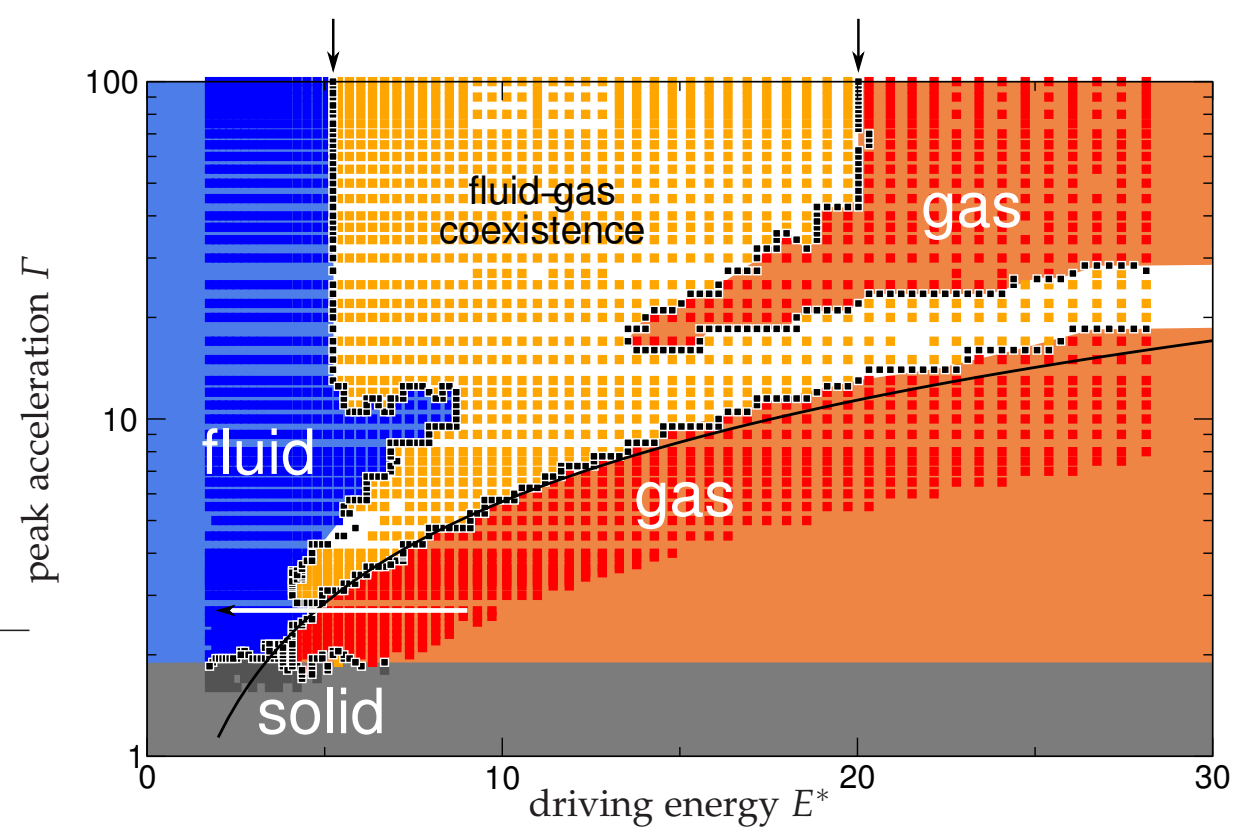

Fig. 3.11 Phase diagram using the thin-thread model and inelastic collisions with a coefficient of restitution of $\varepsilon=0.8$. All other parameters are the same as used for Fig.3.9. The fluid-gas coexistence regions induced by the capillary interaction and the inelastic collision start to merge. The solid black line states where the amplitude is equal to the diameter of one particle. In the inelastic case, the inflection point of the granular temperature is used to detect the phase boundary. The temperature change along the white line is shown in appendix 3.A.1.2.

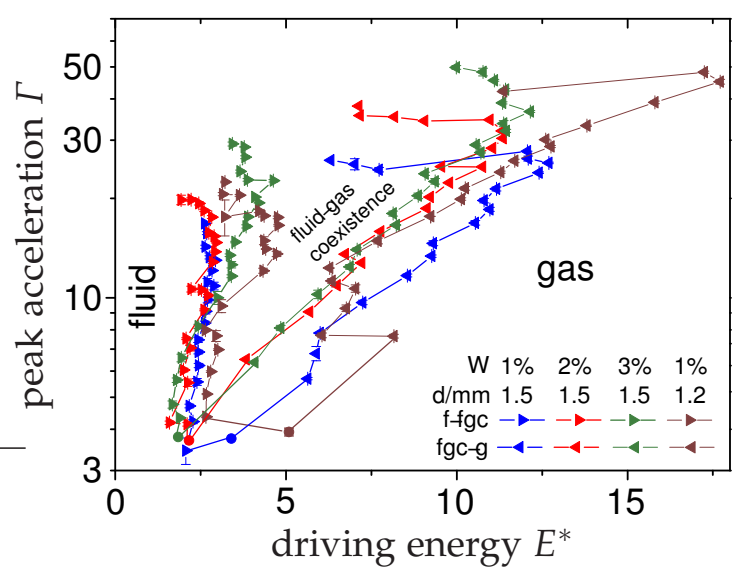

Fig. 3.12 Phase diagrams for water-wetted glass spheres at different liquid content $W$ and particle diameters $d$ in the $\Gamma$ - $E^{*}$ plane, where $E^{*}$ is the driving energy rescaled by the rupture energy of capillary bridges. Heights of the granular sample and the container are $h=3.6 \mathrm{~mm}$ and $H=6.6 \mathrm{~mm}$ correspondingly. Experiment performed by Kai Huang. 


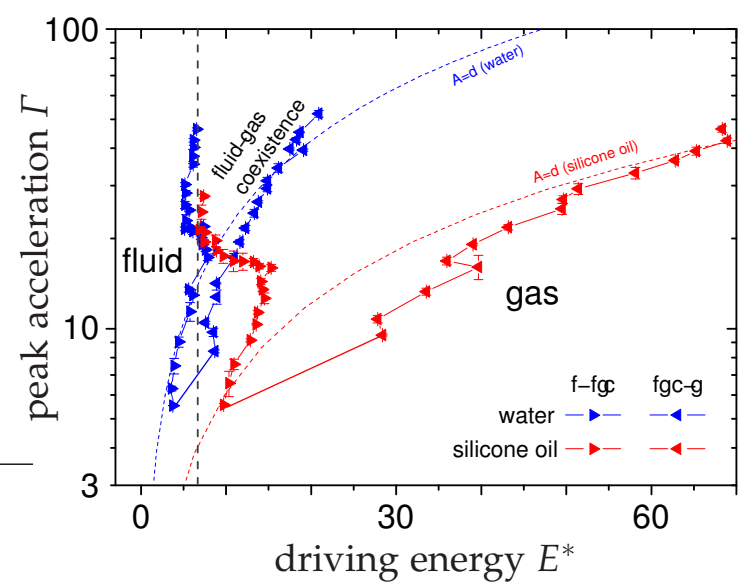

Fig. 3.13 Phase diagram of $1 \mathrm{~mm}$ glass spheres wetted by water and silicone oil in $\Gamma$ - $E^{*}$ plane. The surface tension of water is more than three times that of silicone oil. Other parameters are: liquid content $W=1 \%, h=3 \mathrm{~mm}$ and $H=9.1 \mathrm{~mm}$. Dashed lines correspond to $A=d$, the gray dashed line serves as a guide to the eye. Experiment performed by Kai Huang.

the f-fgc transition is mainly governed by the sample itself. Furthermore, it is rationalized qualitatively how the dependence of the $\mathrm{f}$-fgc transition upon the height of the sample comes about. As it is illustrated in Fig.3.15, the shift of the f-fgc transition line may be due to the increased number of collisions involved in the injected energy's travel from the container bottom (where it is injected) upwards through the sample. If the average number of collisions required for the energy to reach the top layer is $n_{c}$, the effective energy which reaches the top layer is $E_{\text {top }}=E_{\mathrm{inj}} \varepsilon^{2 n_{c}}$, where $\varepsilon$ is the restitution coefficient of the collision between particles, and $E_{\text {inj, }}$, is the energy injected through collisions between bottom layers of the sample and the container. If the energy of the top layer exceeds the capillary bridge energy, $E_{\mathrm{top}}>E_{\mathrm{cb}}$, particles on top layers will be driven into a gaseous phase. For larger sample heights, $h$, the dissipation of energy within the sample increases and therefore more energy injection is needed to meet the criterion $E_{\text {top }}>E_{b}$. This may explain the shift of the fluid-coexistence (f-fgc) transition line to a higher value at increased $h$.

To support this scaling, the critical driving energies obtained by simulations for both energy driven phase boundaries, indicated by the arrows on top of Fig.3.11, are shown in Fig.3.16(a) as a function of the coefficient of restitution, $\varepsilon$. As shown for the f-fgc transition in Fig. 3.16(b), the average number of particle collisions per wall collision, $n_{c}$, is changing with $\varepsilon$. It is reasonable to assume that each layer of particles collides at least once per oscillation cycle of the driving with the layer above, such that $n_{c}$ is expected to be larger than the number of intra-layer collisions, $n_{\mathrm{ilc}}$, which is $n_{\mathrm{ilc}}=n_{L}-1=2$ in the simulations shown in Fig.3.16(a), where $n_{L}$ is the number of layers ${ }^{5}$. As seen in Fig. 3.16(b), the values of $n_{c}$ are safely larger than $n_{\text {ilc }}=2$ (red line), but still in the same order of magnitude, which is a reasonable result consid-

5. The summand minus one is a result of the elastic collision with the bottom. 


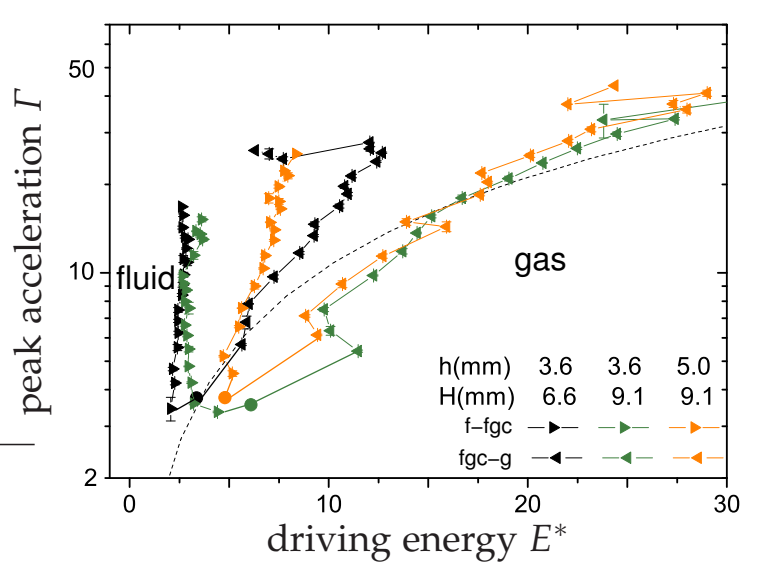

Fig. 3.14 Phase diagram for different sample height $h$ and container height $H$ for $1.5 \mathrm{~mm}$ glass spheres. The samples are wetted by water with liquid content $W=1 \%$. Experiments performed by Kai Huang.

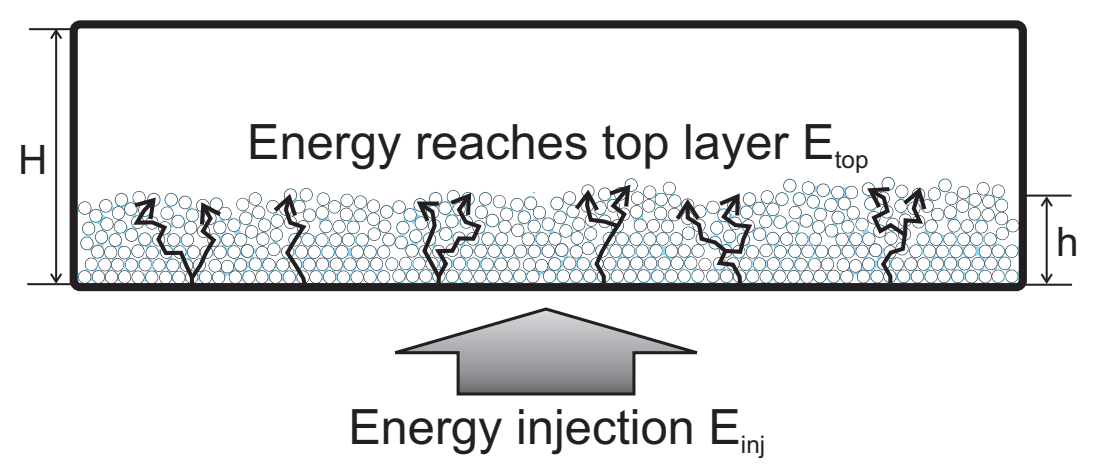

Fig. 3.15 A sketch showing how energy injection is transmitted upwards through collisions between neighboring particles. $h$ and $H$ are height of the sample and the container correspondingly. Sketch provided by Kai Huang. 
(a)

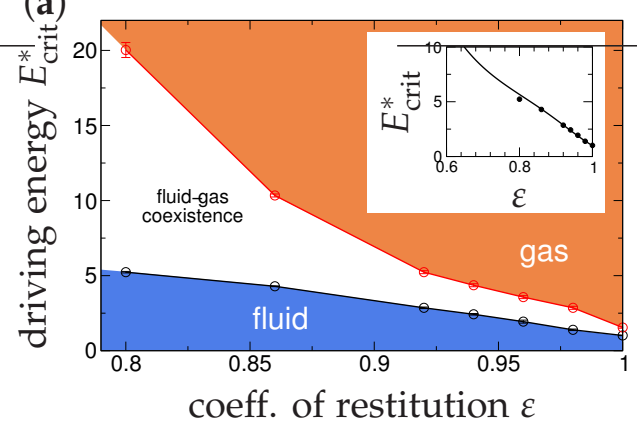

(b)

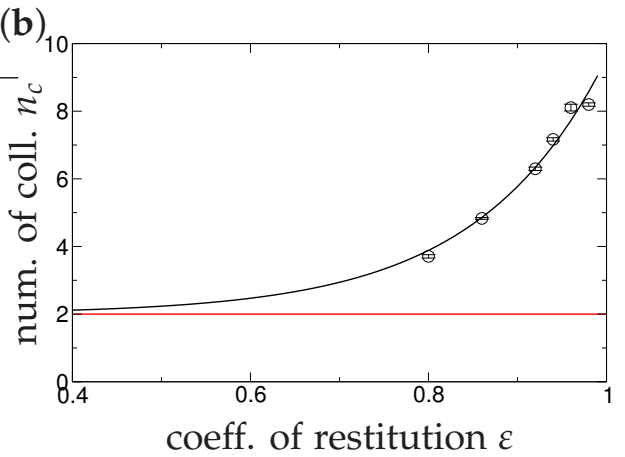

Fig. 3.16 (a) The critical driving energies (arrows on top of Fig.3.11) for both energy driven phase transitions ( $f-f g c$ in black and $f g c-g$ in red) are shown as a function of the coefficient of restitution. The points are connected with lines and serve as guide to the eyes. The inset shows again the simulation results for the f-fgc transition (filled circles) and additionally Eq. (3.1) (solid line) which is obtained as explained in the text. The theoretically predicted curve also shows the expected behavior that $E_{\text {crit }}^{*}$ goes to infinity as $\varepsilon$ approaches zero. (b) The upper bound for the average number of collisions, $n_{\mathcal{c}}$, as obtained by simulations (circles) is in a reasonable order of magnitude and safely larger than the number of intra-layer collisions, $n_{\text {ilc }}=n_{L}-1=2$,(red line), which is a lower bound for the system under study, where $n_{L}$ is the number of layers. The black line is a fit to the data with $n_{c}(\varepsilon)=A_{1} \exp \left(A_{2} \varepsilon\right)+n_{\text {ilc }}$ leading to $A_{1}=7.3 \times 10^{-3}$ and $A_{2}=6.95$. For small coefficients of restitution $n_{c} \approx n_{\text {ilc }}$ seems to be a reasonable approximation.

ering the crudeness of the assumptions made. The black curve in Fig.3.16(b), which is a fit to the data, shows that the average number of particle collisions per wall collision, $n_{c}$, approaches the number of intra-layer collisions, $n_{\mathrm{ilc}}$, for low coefficients of restitution.

The results for the scaling of the f-fgc phase boundary can be summarized in a simple formula, which is valid in the whole range of simulations performed. The critical driving energy, $E_{\mathrm{f}-\mathrm{fgc}}^{*}$, for this transition is found to be

$$
E_{\mathrm{f}-\mathrm{fgc}}^{*}\left(D, \varepsilon, n_{L}\right)=\frac{n_{\mathrm{Kn}}(D)}{\varepsilon^{2 n_{c}\left(\varepsilon, n_{L}\right)}} E_{0}^{*},
$$

where $D$ is the dimension of the system, $\varepsilon$ the coefficient of restitution, $n_{L}$ the number of particle layers, $n_{\mathrm{Kn}}(D)$ the Kissing number (which is 2, 6, 12, 24 in one, two, three and four (Musin, 2008) dimensions respectively), $E_{0}^{*}=1 / 6$ and $n_{c}\left(\varepsilon, n_{L}\right)=$ $A_{1} \exp \left(A_{2} \varepsilon\right)+n_{L}-1$ with the numerically obtained constants $A_{1}=7.3 \times 10^{-3}$ and $A_{2}=6.95$.

\subsubsection{Scaling of the $f g c-g$ transition}

The behavior of the fgc-g transitions is even more complex. This line consists of two branches. The branch at large driving acceleration, $\Gamma$, is dominated by the capillary bridge though shifted by inelastic collisions. The branch at lower driving accelerations $\Gamma$, which is studied here, tends to follow the curve where the vibration amplitude equals a constant critical value, $A_{\text {crit }}$. The precise critical amplitude 
depends on the coefficient of restitution as well as the height of the container, as mentioned above. This may be termed 'non-universal' behavior. Turning back to Fig. 3.13, it can be seen that the transition line between the fgc and g phases seems to be dominated by geometric effects, as here the equality of $A$ and $d$. Simulations reveal in Fig. 3.17 that, as the coefficient of restitution is decreased, the critical amplitude, $A_{\text {crit }}$, of the driving, at which the lower branch of the fgc-g transition occurs, increases. For $\varepsilon=0.8, A_{\text {crit }} \approx d$ is obtained, which is the value obtained similarly in the experiment shown in Fig. 3.13. This aspect needs further investigation.

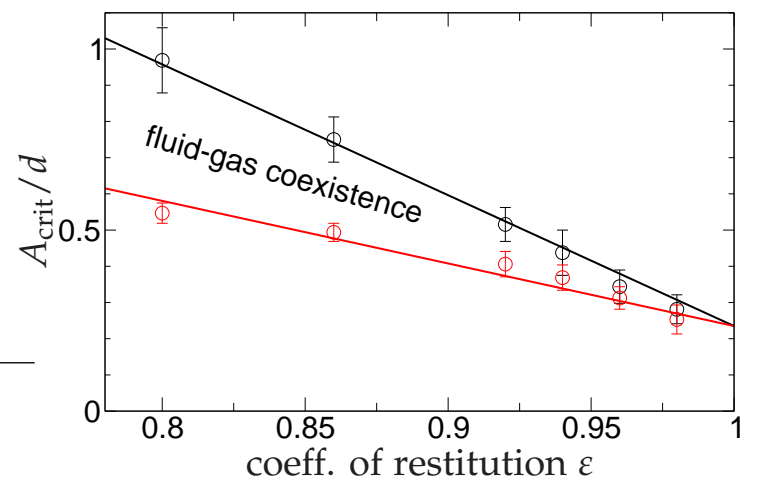

Fig. 3.17 Critical amplitude of the driving, $A_{\text {crit }}$, where the lower branch of the fgc-g phase transition sets in. (It is noted that $\Gamma$ is increasing by decreasing the amplitude.) The solid black line is a fit of the phenomenological function $A_{\text {crit }} / d=-3.61(\varepsilon-1)+0.24$ while the red line is a fit resulting in

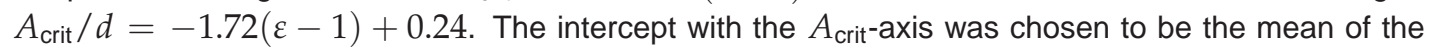
two independently obtained intercept values from a fit.

\subsubsection{Narrower particle size distribution}

Finally, the influence of a lower polydispersity to the phase diagram is studied. The polydispersity of the particles, which safely prevented crystallization, was $\sigma_{p}=0.06$ and $\sigma_{p} \approx 0.05$ in simulations and experiments, respectively. To allow partial crystallization in the granular sample, the polydispersity of the particles is reduced to $\sigma_{p}=0.02$ and $\sigma_{p} \approx 0.02$ in simulations and experiments, respectively.

Fig. 3.18(a) is a snapshot of a three-dimensional simulation at driving parameters $E^{*}=1.13$ and $\Gamma=100$, which shows a solid-fluid coexistence, where a crystallized solid (blue) is in coexistence with a fluid phase (orange). The color code is streched to better reveal the difference such that orange or red indicates a fluid, where the average kinetic energy is in the order of the capillary bridge energy. In the shadow beneath the sample the crystallization is also visible. The snapshot in Fig. $3 \cdot 18(\mathrm{~b})$ shows a simulation at higher driving energy $\left(E^{*}=1.35\right)$, which indicates, that the size of the fluid region is increasing because larger parts of the crystallized granular matter can be melted. Similar behavior is obtained in experiments as shown by Fig.3.19. The figure shows an average over 80 successive snapshots, which corresponds to a real time period of $0.53 \mathrm{~s}$. It can be seen that there exist immobile, 
crystalline-ordered particles of the solid phase as well as mobile, unordered particles (which appear fuzzy in the image) of the fluid phase.

The phase diagram, as obtained by experiments, is shown in Fig.3.21. A region is visible where the solid-fluid coexistence occurs. It is noted that the solid to solidfluid coexistence (s-sfc) boundary still is a horizontal line at $\Gamma \approx 2.0$ and therefore driven mainly by force, while the sfc- $f$ transition is a vertical line and therefore is dominated by the driving energy. The phase diagram shown in Fig. 3.20 is obtained by simulations of a three-dimensional system. The solid-fluid coexistence region is clearly visible. It can be seen that the s-sfc transition splits into two branches, one which is, in accordance to the experiments, a horizontal line at $\Gamma \approx 1.65 \pm 0.05$ and a second which is, at sufficiently high $\Gamma$ a vertical line at $E^{*}=0.80 \pm 0.06$. The latter one is not accessible in the experiment due to limitation of the apparatus. Also the lower branch of the s-f transition shows a small region where a sfc state can be observed. The most prominent sfc-f transition is, in accordance with the experiment, a vertical line at a critical driving energy $E^{*}=1.35 \pm 0.08$, which is within the measurement error not far from the value $E^{*}=0.88 \pm 0.20$ obtained in the experiment.

Interestingly, the change of the polydispersity of the particles from $\sigma_{p}=0.06$ to 0.02 in simulations did not noticeably change the f-fgc phase boundary. In the experiment, the phase boundary similarly did not change significantly be seen by comparing figures 3.21 and Fig. $3.8(\mathrm{~b})$. The critical driving energy for this transition to occur is $E^{*}=4.36 \pm 0.14$ in both cases. The sfc-f transition, however, significantly depends on the polydispersity. At a polydispersity of 0.02 the system clearly shows a solid-fluid coexistence and the transition occurs at $E^{*}=1.35 \pm 0.08$ as mentioned before. Increasing the polydispersity to 0.06 makes the solid-fluid coexistence invisible and the corresponding s-f transition occurs already at $E^{*}=1.06 \pm 0.07$.

A solid-fluid coexistence is also obtained in thermally equilibrated systems of a pure hard-core gas without any attractive forces (Laird and Davidchack, 2005, and references therein). In the equilibrium case there exists an interfacial energy between the solid and fluid phase which can be understood in terms of the free energy arising from entropy. As the equilibrium hard sphere system does not have an intrinsic energy scale, the solid-fluid transition only depends on density. Therefore, it is surprising that the sfc-f phase transition in wet granular matter studied here occurs at a certain driving energy. This indicates that this transition is again mainly determined by the capillary bridge interaction.

\subsubsection{Effect of inelasticity in dilute systems}

As inelasticity was added to the two-dimensional simulations of wet granular matter, a third coexistence region, as shown in Fig. 3.9, appeared. Simulations of dry granular matter, where dissipation is caused only by inelastic collisions, also showed this coexistence region. This demonstrates that it is caused by inelasticity (Fig. 3.10). This coexistence region seemed to occur at a critical driving amplitude which depends on 


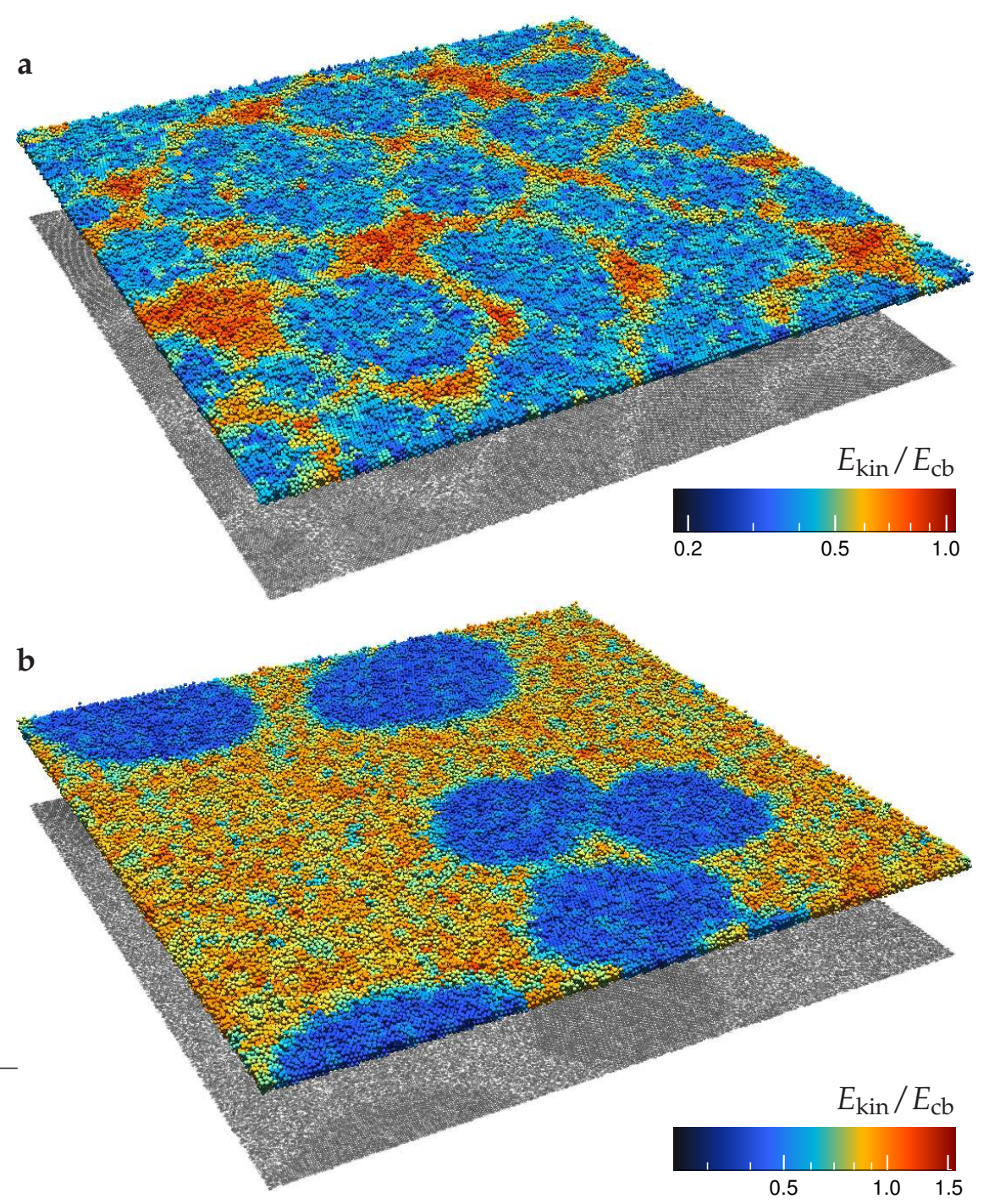

Fig. 3.18 Snapshots of three-dimensional simulations at driving parameters $\Gamma=100$ and $E^{*}=1.13$ (a) or $E *=1.35$ (b). A solid-fluid coexistence is observed where crystallized solid parts (blue) coexist with a fluid phase (red). The color code is stretched to better reveal the difference such that orange or red indicates a fluid where the average kinetic energy is in the order of the capillary bridge energy. In the shadow beneath the sample the crystallization is also visible. Due to the increased driving energy in (b), a larger amount can be melted to the fluid phase compared to snapshot (a). The system contains $1.2 \times 10^{5}$ particles and the system dimensions are $9 d \times 192.5 d \times 192.5 d$. The polydispersity is $\sigma_{p}=$ 0.02 and the inelasticity is $\varepsilon=0.96$. Other parameters are the same as in previous two-dimensional simulations (cf. Fig. 3.9). 


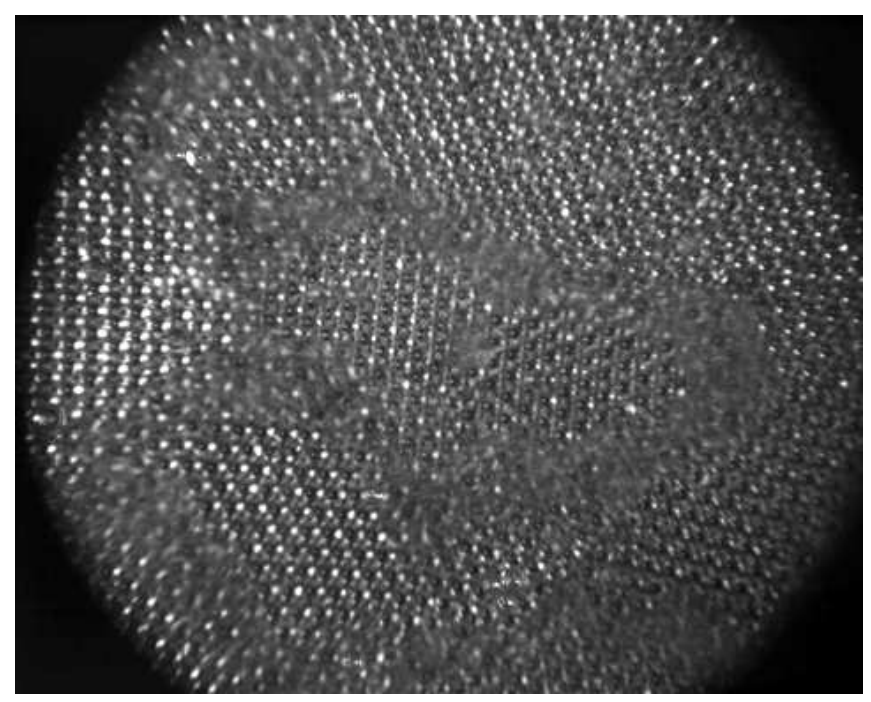

Fig. 3.19 The image shows an average over 80 successive snapshots (corresponding to $0.53 \mathrm{~s}$ in real time). Immobile, crystallized regions (solid phase) coexist with mobile granular particles (fluid phase). Mobile particles look fuzzy in the image due to the averaging, while immobile particles are visible as a hexagonal packing with bright spots from light reflection. The experiment is performed at a driving frequency of $f=150 \mathrm{~Hz}$ and driving acceleration $\Gamma=7.42$. Experiment performed by Kai Huang.

the coefficient of restitution, as shown in Fig. 3.17. However, this coexistence region could not clearly be observed in the three-dimensional simulations of wet granular matter presented in the phase diagram in Fig. 3.20, suggesting that this could possibly be an effect caused by dimensionality. In order to check this hypothesis, three-dimensional simulations were performed with the spatial extension of the system strongly reduced in one lateral dimension, to only $L_{y}=9$ particle diameters. $L_{y}$ is the system width in the second lateral direction with periodic boundary conditions. (The number of particle layers was kept constant and all other parameters are similar to the ones used in Fig. 3.10.) The phase diagram obtained by these simulations is shown in Fig. 3.22. As expected, the coexistence region is visible again, but only in a parameter range much smaller than in the two-dimensional system, cf. Fig.3.10. This apparently supports the hypothesis of the effect being caused by dimensionality. Therefore, more simulations were performed where the lateral system size in the third dimension is doubled to $18 d$. To support the hypothesis that the coexistence is caused by dimensionality, the expectation is that the parameter range, in which the coexistence is visible, is reduced. This could not be assessed if the data were presented in the same resolution as the phase diagram shown in Fig.3.22. Therefore, only the minimum and maximum granular temperature, which serves as an order parameter to detect coexisting phases at a peak driving acceleration $\Gamma=10.0$ is shown in Fig.3.23. This is for a system with a lateral extension of $L_{y}=9 d$ (black lines) and $L_{Y}=18 d$ (red lines), where circles denote the maximum and squares the minimum value. It can be seen that the range of parameters, in 


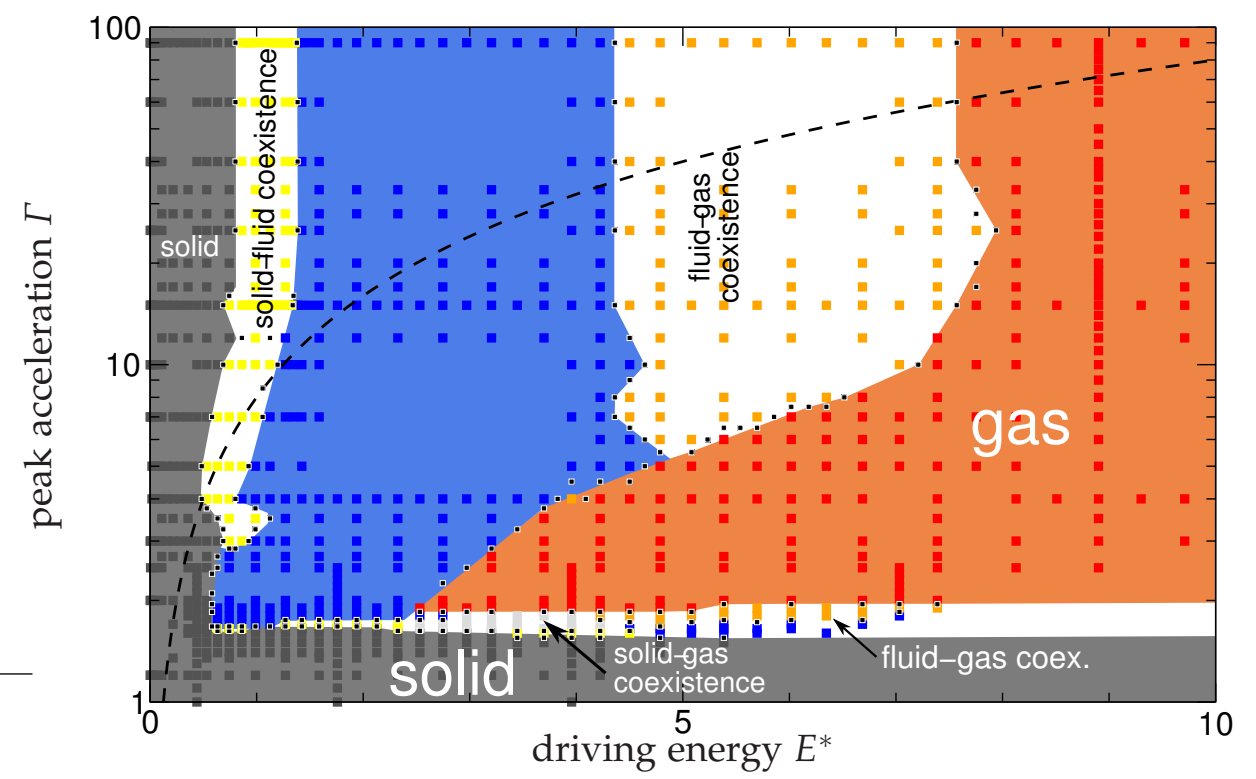

Fig. 3.20 Phase diagram of $1.2 \times 10^{5}$ spheres in three dimensions using the thin thread model, with a coefficient of restitution $\varepsilon=0.96$ for collisions between particles. The polydispersity is reduced to $\sigma_{p}=0.02$ such that the solid-fluid transition is accompanied by a solid-fluid coexistence. The dashed black line is where the driving amplitude is equal to the critical rupture separation of the capillary bridges $A=s_{\text {crit }}$ and seems to slightly influence the solid-fluid coexistence at low peak accelerations, $\Gamma$. The fluid-gas coexistence, which is presumably caused by the inelasticity of particle collisions, cannot be observed in this phase diagram. Further simulations are shown in Sec.3.3.2.6 in order to examine the reason for its disappearance. The box dimensions are $9 d \times 192.5 d \times 192.5 d$, while other system parameters are the same as in previous two-dimensional simulations (cf. Fig. 3.9).

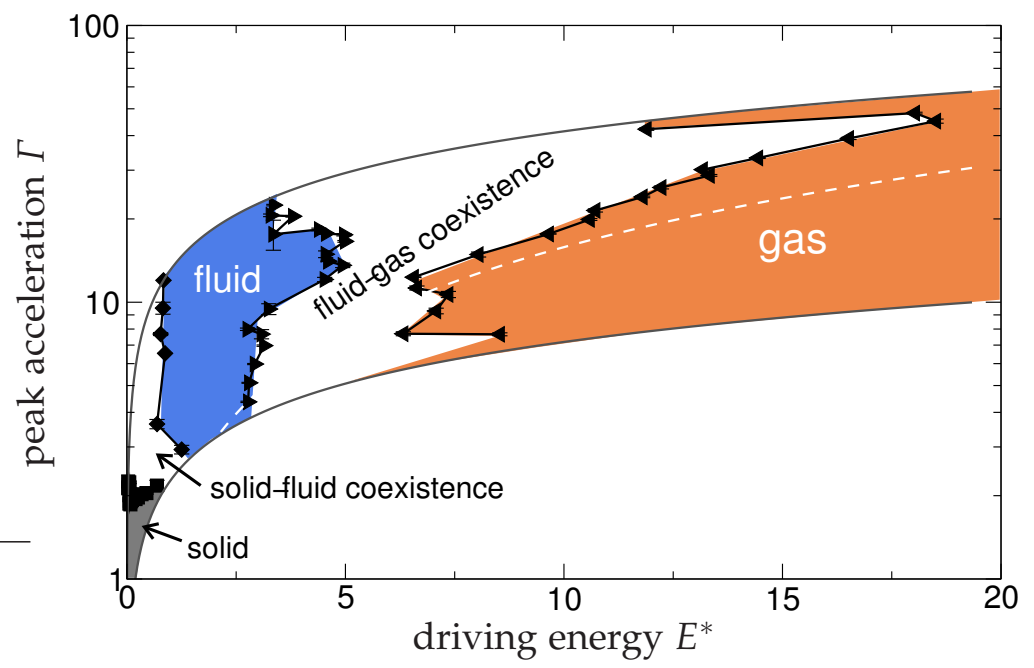

Fig. 3.21 Phase diagram for water-wetted glass spheres of diameter $1.2 \mathrm{~mm}$ (Whitehouse scientific GP1215) with a polydispersity of about $\sigma_{p}=0.02$ in the $\Gamma$ - $E^{*}$ plane. A finite region can be observed where a solid-fluid coexistence emerges. The liquid content is $W=1 \%$ and the dashed line corresponds to the curve, where the amplitude $A=d$. Experiment performed by Kai Huang. 


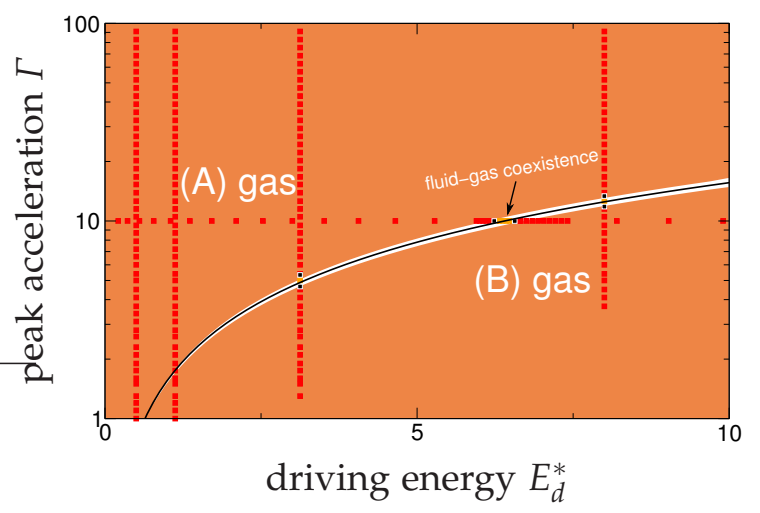

Fig. 3.22 Phase diagram of three-dimensional dry granular matter with $E_{\mathrm{cb}}=0$ and coefficient of restitution $\varepsilon=0.96$. Except for the dimensionality and therefore the system width of $L_{y}=9 d$ in the second lateral direction and an increased particle number of $2.16 \times 10^{4}$ particles being in the system, all other parameters are identical to the ones used for the phase diagram in Fig.3.10. The axis of the driving energy is normalized as $E_{d}^{*}=\frac{\frac{1}{2} m(A \omega)^{2}}{m g d}$, where $m$ is the mass of one particle, $g$ is the acceleration due to gravity and $d$ is the mean particle diameter. The solid black line denotes where the amplitude of the driving equals 1.28 times the mean diameter of the particles. It is visible that the coexistence region is very narrow but is clearly detectable. It divides the gas-like region into two regimes (A) and (B).

which the coexistence region appears, does not change (within the resolution of the simulated points). This becomes especially visible in the inset, which is a zoom to the coexistence region. Therefore, it can be concluded that this coexistence region is not an artifact of the two-dimensional system, as previously assumed. A snapshot of such a three-dimensional simulation of dry granular matter in a coexistence state can be seen in Fig. 3.24.

It is noted that the absolute value of the difference between the maximum and minimum areal density, $\Delta n_{\text {area }}$, in a coexistence state strongly depends on the underlying mechanism (inelasticity or capillary bridges) which causes the coexistence. Comparing Fig. $3 \cdot 31$ (which shows $\Delta n_{\text {area }}$ for a coexistence which is purely caused by the interaction of capillary bridges) with Fig. $3 \cdot 25$ (which shows $\Delta n_{\text {area }}$ for a coexistence which is purely caused by inelastic collisions), it can be seen that the maximum value of $\Delta n_{\text {area }}$ which is reached in these figures is $\max \left(\Delta n_{\text {area }}\right) \approx 0.68$ and $\max \left(\Delta n_{\text {area }}\right) \approx 0.29$, respectively. Thus, the maximum density difference which is reached in a coexistence state is much higher if it is caused by capillary bridges than if it is caused by inelastic collisions ${ }^{6}$.

Another aspect detectable in Fig. 3.23 is that for low driving energies $E_{d}^{*}$ the granular temperature increases linearly with $E_{d}^{*}$ as predicted in the limit of high frequency and vanishing amplitude for a non-moving wall, which only injects a certain amount of energy per collision with the wall (Herbst et al., 2004). Therefore, the approxima-

6. This phenomenon can also be observed in experiments of wet granular matter, where the two coexistence regimes caused by capillarity and inelasticity therefore even could be distinguished (Fuchs and Wallenhorst, 2008). 


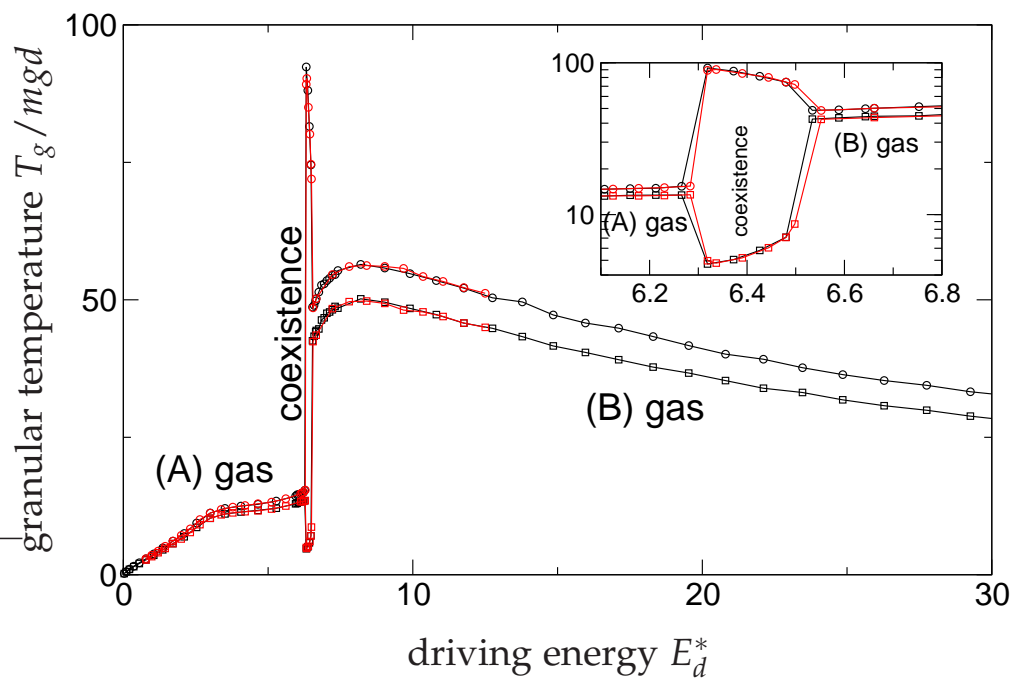

Fig. 3.23 Minimum (squares) and maximum (circles) granular temperature $T_{g}$ is shown as a function of the driving energy $E_{d}^{*}$ for several three-dimensional simulations. The system differs in the system size in the second lateral direction which is $L_{y}=9 d$ (black curves) and $L_{y}=18 d$ (red curves). The areal density is also kept constant such that the system contains $2.16 \times 10^{4}$ (black) and $4.32 \times 10^{4}$ particles (red). All other parameters are the same as in Fig.3.22. It can be seen that the two different systems do not show a significant difference. Especially the inset, which is a zoom to the coexistence region, clearly demonstrates that, within in the range of simulated values, the size of the coexistence region does not change. Therefore, it is concluded that it is not an effect of dimensionality. A coexistence in this graph is characterized by a large difference between the minimum and maximum granular temperature in the system, much larger than obtained by usual fluctuations. This also shows that the coexistence is not a coexistence of two gas phases (in the notion of this thesis) but is a fluid-gas coexistence. Note the logarithmic scaling of the temperature axis in the inset.

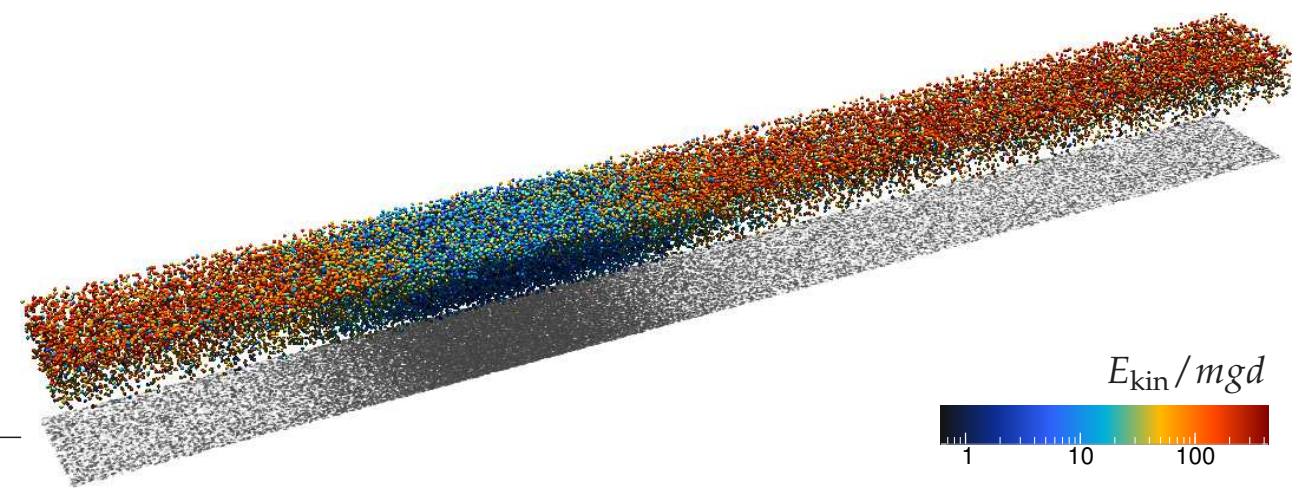

Fig. 3.24 Snapshot of a three-dimensional simulation of dry granular matter with a coefficient of restitution $\varepsilon=0.96$ for collisions between particles while wall collisions occur elastically. The system contains $4.32 \times 10^{4}$ spherical particles and the system size is $400 d \times 18 d \times 9 d$. The driving parameters are $\Gamma=10.0$ and $E_{d}^{*}=6.4$. Polydispersity of the particles is $\sigma_{p}=0.06$. 


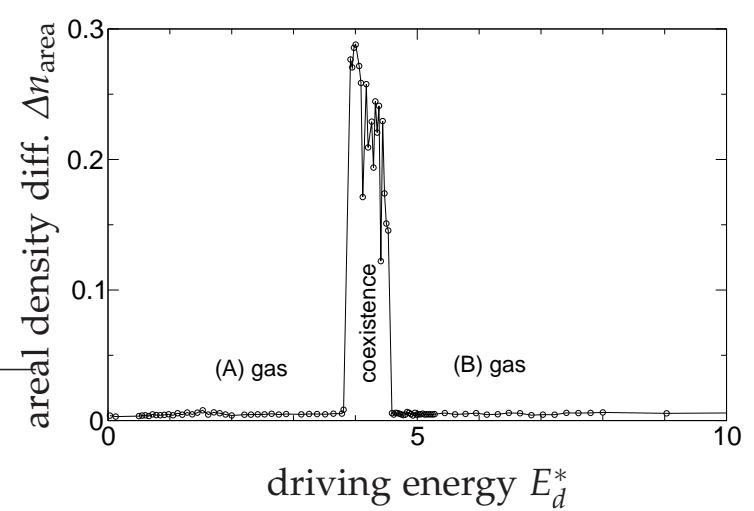

Fig. 3.25 The difference in the areal density, $\Delta n_{\text {area }}$, is plotted for the two-dimensional system shown in Fig.3.10. By comparing the data presented in Fig.3.31 (which shows $\Delta n_{\text {area }}$ for a coexistence which is purely caused by the interaction of capillary bridges) with the data in the present figure (which is a simulation of dry granular matter only undergoing inelastic collisions), it can be seen that the difference in the areal densities are more than a factor of two smaller than in the fluid-gas coexistence, which is caused by the capillary bridges shown for a two-dimensional simulation of elastically colliding particles in Fig.3.31. This seems to be due to the lower density of the dense phase in the dry case compared to the wet case, where capillary bridges impose attractive forces. The values are taken at $\Gamma=10.0$.

tion of a non-moving wall seems to be applicable also for the case of moving wall in the regime (A) of low driving energies, $E_{d}^{*}$. However, for larger driving energies, $E_{d}^{*}$, (where the driving amplitude is increasing, while at the same time the driving frequency is decreasing $(\Gamma=$ const.) ), a simple linear relation between the driving energy and the mean granular temperature cannot be found. In fact it is observed in Fig. 3.23 that above a critical driving energy the mean granular temperature is even decreasing with increasing driving energy (regime (B)). This suggests the appearance of a second mechanism of energy injection where it is not the kinetic energy of the wall motion that determines the mean granular temperature but is mainly the peak acceleration of the wall. This can be seen in the linear increase of the granular temperature, $T_{g}$, with the peak acceleration, $\Gamma$, in regime (B) of Fig. 3.27. Thus, the difference in the regime (A) and (B), where in both cases a gas state can be found, seems to be the mechanism of energy injection. Regime (A) is dominated by the kinetic energy of the wall motion whilst regime (B) is mostly ${ }^{7}$ dominated by the peak acceleration of the driving.

Therefore, the result shown in Fig. 3.23 demonstrates the limitations of the nonmoving wall approximation. The breakdown of the linear relation $T_{g} \propto E_{d}^{*}$ and the change in the energy injection mechanism seems to be crucial for the occurrence of coexisting states, as these were only observed in the regime where the energy injection mechanism seems to change. This would also explain why this self-organized phase separation in dry granular matter was not observed in previous numerical studies using non-moving walls. As the effect of coexisting phases, which is pre-

7. The granular temperature in Fig. 3.23 in regime (B) is not constant even though $\Gamma$ is constant. This shows that the actual dependence is more complex and needs a higher order correction. 
sented here, seems to occur at a certain well-defined amplitude (cf. for example Fig. 3.22), this supports the assumption that spatially moving walls are inevitable for its occurrence. This underlines at the same time the importance of using real moving walls also in event-driven simulations disregarding the higher implementation effort. An efficient implementation of sinusoidally moving walls is presented in appendix B to this work.

The question which still remains open is the shrinking of the range of parameters in which the coexistence could be observed when going from the two-dimensional (Fig.3.10) to the three-dimensional simulations (Fig.3.22) and the main parameter which influences its occurrence. In Fig. 3.26 the granular temperature of threedimensional simulations of two different systems are shown, where the dashed line is the curve already shown above (red lines in Fig. 3.23) and the solid line refers to almost identical simulations except for the number of particles, which are reduced to $2.16 \times 10^{4}$ particles. This means that only half the amount of particles were used while the container dimensions are the same. Thus, the overall packing fraction was reduced from $\phi / \phi_{\max }=0.118$ (red) to $\phi / \phi_{\max }=0.059$ (black), where $\phi_{\max }=0.74$ is the maximum packing fraction in three dimensions. It is clearly visible that the range of parameters in which the coexistence state can be observed is increasing in size if the density is decreased. Therefore, it is concluded that one of the main system parameters, which determines the visibility of the coexistence region is the average particle number density in the system, as the coexistence region in the phase diagram is increasing for dilute systems. This is basically supported by experiments using wet granular matter which were presented Fig.3.14. There it can be seen that the range of parameters in which the coexistence is observed is increasing by decreasing the average particle number density (black or orange curve (high density) to the green curve (low density)) similar to what is observed in simulations of dry granular matter. This suggests that the coexistence in experiments which ends at a critical driving amplitude are caused or at least supported by inelasticity and merged with the coexistence which is caused by the capillary interaction. It is further noted that therefore also the critical amplitude at which the coexistence occurs additionally depends on the average particle number density.

It is now confirmed by simulations and partially by experiments that the average particle number density is the main system parameter (ahead of the coefficient of restitution $\varepsilon$, cf.Sec.3.3.2.4) which determines the size of the parameter range in which the coexistence is visible. As the number of layers of particles in the system was kept constant when going from two (Fig. 3.10) to three dimensions (Fig. 3.22), it is observed that the relative areal density $\phi / \phi_{\max }$, (where $\phi_{\max }=0.91$ and $\phi_{\max }=0.74$ in two and three dimensions respectively) even decreases from $\phi / \phi_{\max }=0.144$ in the two-dimensional phase diagram to $\phi / \phi_{\max }=0.118$ in the three-dimensional phase diagram. This contradicts at first glance the previous findings, as decreasing particle number density should lead to an increase of the parameter range in which the coexistence is visible. However, at the transition from twoto three-dimensional simulations (cf. Fig.3.10 with Fig.3.22) the parameter range in 


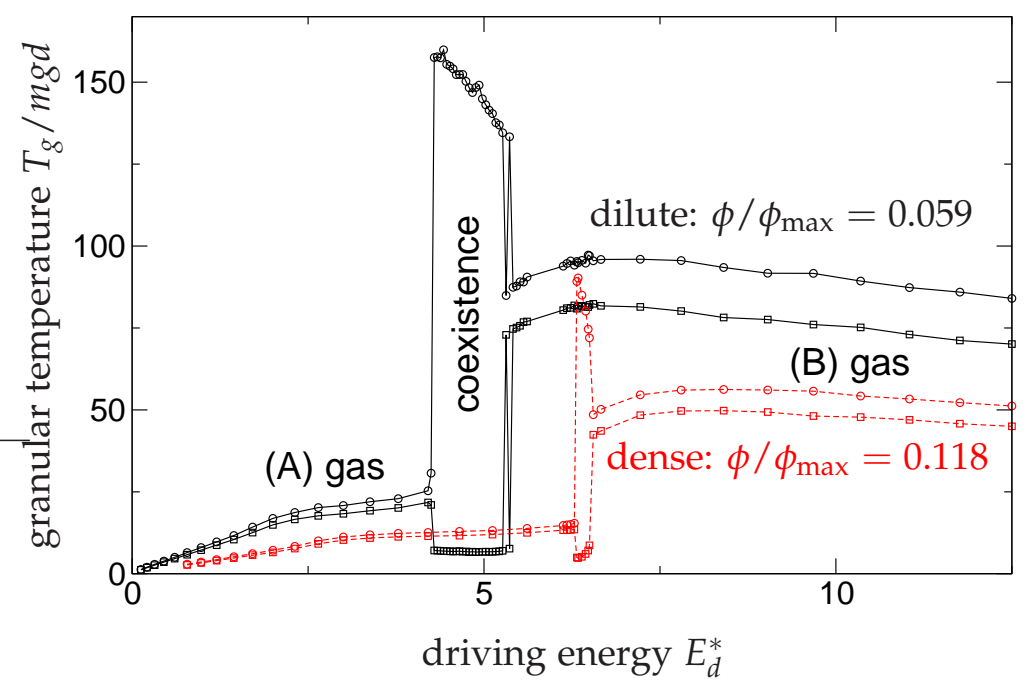

Fig. 3.26 Minimum (squares) and maximum (circles) granular temperature $T_{g}$ is shown as a function of the driving energy $E_{d}^{*}$ for two different systems. The red dashed line is the system shown in Fig. 3.23 (red line). The solid black line is a similar system where only the number of particles is reduced by a factor of two to $2.16 \times 10^{4}$ particles. The overall packing fraction thus was reduced from $\phi / \phi_{\max }=0.118$ (red) to $\phi / \phi_{\max }=0.059$ (black), where $\phi_{\max }=0.74$ is the maximum packing fraction in three dimensions. It is clearly visible that the range of parameters in which the coexistence can be observed is decreasing by increasing density. Therefore, it is concluded that the main system parameter is the areal density in the system.

the three-dimensional phase diagram where the coexistence is observed is shrinking - almost vanishing - even though the relative particle number density in the system reduces. However, it can be seen in Fig. 3.26 that the granular temperature in general is higher in the system with lower density (black curves), which is reasonable as the dissipation in a dilute granular gas is proportional to the areal density (Brilliantov and Pöschel, 2004). It seems that higher granular temperature leads to a larger region in which the coexistence can be observed. This would explain the decrease in the range of parameters, where the coexistence can be observed going from two to three dimensions, because the increase in dimensionality increases the number of degrees of freedom. If the kinetic energy which is injected into the pile remains the same, then the granular temperature in three dimensions reduces to $2 / 3$ of the twodimensional value, which is approximately confirmed by simulations (not shown here). These findings explain why the third coexistence region caused by inelasticity was not observed in the phase diagram obtained by simulations of wet granular matter in Fig. 3.20.

The results shown in this subsection demonstrate again the importance of using moving walls in simulations in order to be comparable with experiments, because this coexistence cannot be observed in the above mentioned limit of vanishing amplitudes of a non-moving wall. Furthermore, when studying phase diagrams of wet granular matter it is desirable to suppress the effects caused by inelasticity. There- 


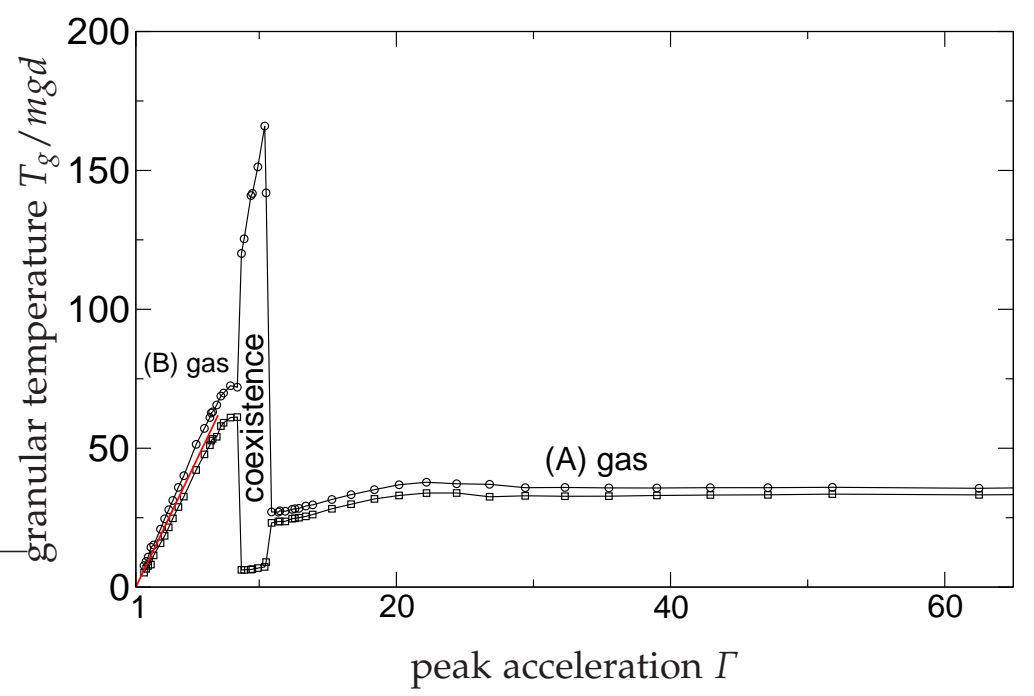

Fig. 3.27 Minimum (squares) and maximum (circles) granular temperature $T_{g}$ is shown as a function of the peak acceleration $\Gamma$ of the driving. The red line at low values of $\Gamma$ is of the form $T_{g} \propto \Gamma-1$. This shows that the granular temperature in the regime (B) increases linearly with $\Gamma$ and suggests that $\Gamma$ mainly determines the granular temperature as it seems that $T_{g}=0$ for $\Gamma \leqslant 1$. It is noted that the quantities on both axes are dimensionless such that the proportionality constant between $T_{g}$ and $\Gamma$ also is dimensionless. For larger peak accelerations (regime $(A)$ ) the granular temperature is independent of $\Gamma$ which is the regime where the granular temperature is determined by the kinetic energy of the driving wall. The system shown here is identical to the dilute system used in Fig.3.26 (black line). The data is shown at constant driving energy $E_{d}^{*}=4.5$.

fore, these results suggest that this can be done by increasing the overall density in the system as the coexistence regime caused by inelasticity then vanishes. This knowledge is inevitable in order to successfully perform experiments either on dry or wet granular matter. The task which remains to be done is to derive theoretically an expression for the energy injection of a sinusoidally moving wall to an inelastic system of particles. Balancing this with the corresponding dissipative term should then show a stationary solution of a coexisting state. However, this is far beyond the scope of the present thesis.

\subsection{Summary and Outlook}

It was shown by comparing experimental studies to simulations taking inelasticity into account that the phase diagram of mechanically agitated wet granular matter shows two universal phase transitions which depend only on few general intensive system parameters. One is the solid-fluid transition which is mainly driven by the applied force scale, which is represented here by the applied acceleration. The position of this transition line is directly given by the adhesive force acting between adjacent grains. The second is the transition between a fluid phase and fluid-gas coexistence, and is driven by the maximum kinetic energy. Its position is given by 
the energy which propagates from the bottom of the pile (where agitation occurs) to the upper surface of the sample, where evaporation and thereby the transition to coexistence is taking place. The relevant energy scale is the energy which is needed to break a capillary bridge between two adjacent grains. Aside from these two transitions, which in the plane spanned by the acceleration and agitation energy are represented by a horizontal and a vertical straight line, respectively, there are as well a number of non-universal features, which mainly show up in the transition from the fluid-gas coexistence to the gas phase. These depend notably on geometric relations like the size of the container, dimensionality and are furthermore strongly dependent on the inelasticity of the grains.

Significant parts of this chapter have been published in Fingerle, Roeller, Huang, and Herminghaus (2008) and Huang, Roeller, and Herminghaus (2009).

\section{A. Order parameters}

An order parameter is a physical quantity which is used to define in which phase a system resides. It is zero in one phase and non-zero in the other phase. If the order parameter is bounded it can be normalized to the standard interval $[0,1]$ (Bergmann and Schaefer, 1998, chap. 38). In a classical fluid dynamics equilibrium system for example the density is used to distinguish the solid, liquid or gas phase from each other. In the granular system, as showed in this section, order parameters are used which are inspired by the granular structure or leant from order parameters known from classical equilibrium systems.

The task of detecting the phase of the system can be subdivided into two parts. First, the ability to distinguish homogeneous phases from each other and second, the ability to detect inhomogeneous phases of two coexisting phases.

\section{A.1. Discrimination of homogeneous phases}

First the discrimination of the homogeneous phases solid, fluid and gas is explained, which were found in the granular system as shown in sec.3.1.2.

\section{A.1.1. Solid-fluid phase boundary}

The solid phase is physically characterized by particles which are at rest and do not move in their center of mass system. This gives rise to a short range order where particles tend, whenever possible, to form hexagonal closed packings which then persists, with all its defects, over time. Due to the technical details of simulating granular particles, however, it is not possible that particles have zero velocity. Therefore, solid in this thesis means that the velocity of the random motion of particles is small compared to other energy scales in the system. This energy scale can be for example the granular temperature in units of the capillary bridge energy (in the case of wet granular matter). The fluid phase is characterized by a vivid movement 
of the particles and the short range order is only visible on very short timescales. These different time scales on which the (short range) order exists is utilized for a dynamic order parameter which is called frequency of location changes.

A Delaunay triangulation ${ }^{8}$ for the positions of the particle centers is performed. Then each Voronoi-cell accounts for the location of one particle and the whole triangulation is therefore valid until one of the particles' centers moves out of its initially calculated Voronoi cell ${ }^{9}$. This particle then changed its location, because it moved into the Voronoi cell of another particle and the Delaunay triangulation is violated and recalculated. The frequency of these site changes, or equivalently the frequency of violations of the Delaunay triangulation is called frequency of location changes, $f_{\mathrm{lc}}$, and serves as the order parameter to discriminate the solid and fluid phase. This, however, is only used in the time-driven MD simulations. In the event-driven MD simulation the granular temperature is still used. It is stressed that the frequency of location changes is a dynamical order parameter which needs information about the time evolution of the system and cannot be calculated out of a single snapshot of the system.

In Fig. 3.28(a) the frequency of location changes is shown for the solid-fluid like phase transition as obtained by changing the peak acceleration $\Gamma$ of the driving. A hysteresis for increasing or decreasing peak acceleration is clearly observed and therefore a subcritical bifurcation can be seen, which is reminiscent of a discontinuous phase transition.

\section{A.1.2. Fluid-gas phase boundary}

To discriminate the fluid from the gas phase, simply the granular temperature, $T_{g}$, is used, which is defined as

$$
T_{g}:=m\left(\left\langle\vec{v}^{2}\right\rangle-\langle\vec{v}\rangle^{2}\right) / k_{B} f_{d}
$$

normalized by the capillary bridge energy $E_{\mathrm{cb}}$, where $m$ is the average mass of an individual particle with velocity $\vec{v}, k_{B}=1$ is 'Boltzmann's constant' and $f_{d}$ the number of degrees of freedom.

In Fig. 3.28(b) the normalized granular temperature is shown for the direct fluidgas like phase transition as obtained by changing the driving energy $E^{*}$, which clearly distinguishes the two phases.

In the case of inelastic collisions the order parameter is slightly modified, because there is no jump in the granular temperature between the fluid and gas state as in the elastic case. Therefore, the inflection point of the granular temperature, $T_{g} / E_{\mathrm{cb}}$, as shown in Fig. 3.29, determines the phase boundary in this case.

8. In this work the package Triangle (written by J. R. Shewchuk, University of California at Berkeley) for calculating the Delaunay triangulation was used.

9. Not the initially calculated absolute positions are used to detect the violation, but only the initially calculated distribution of Voronoi cells which move along with their particles forming the edges is considered. 


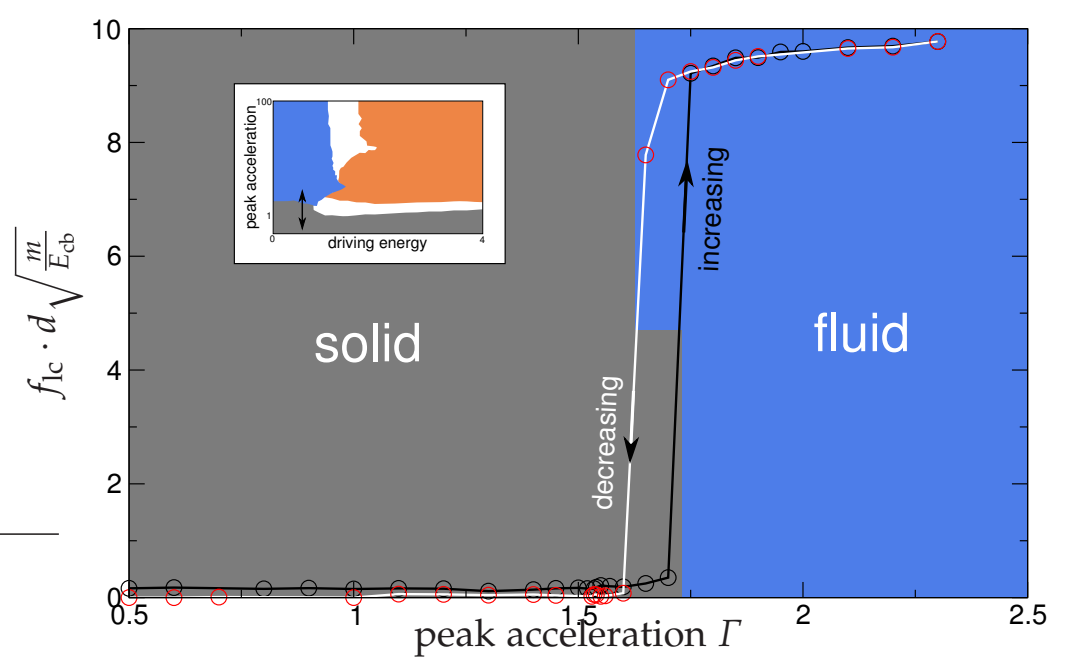

(a) Frequency of location changes as the peak acceleration $\Gamma$ of the sinusoidal shaking is varied. A hysteresis is clearly obtained, which indicates a subcritical bifurcation and is reminiscent of a discontinuous phase transition. The driving energy is $E^{*}=0.63$.

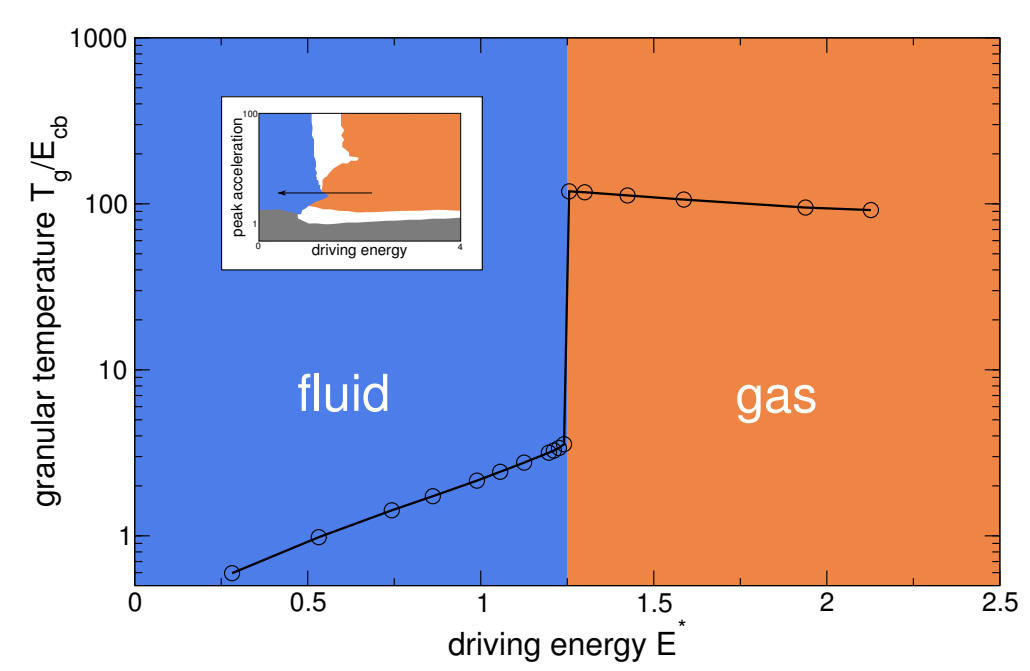

(b) Normalized granular temperature as the driving energy $E_{\mathrm{cb}}$ of the sinusoidal shaking is varied. The jump in the order parameter indicates a subcritical bifurcation and is reminiscent of a discontinuous phase transition, which is hysteretic. The peak acceleration of the driving is $\Gamma=4.0$

Fig. 3.28 Order parameters for the detection of phase boundaries between homogeneous phases (a) solid-fluid and (b) fluid-gas in a time-driven MD simulation of 1200 two-dimensional, elastically colliding spheres. Each inset shows the full phase diagram where the actual parameter scan is denoted, including the direction of the scan (arrows). The system parameters are as follows: Width of the system is 400 particle diameters, the height is 9 particle diameters, $s_{\text {crit }} / d=0.0711, E_{\mathrm{cb}}=1.1374$, average particle diameter is 4.0 and the mass of each particle is $m=1$. 


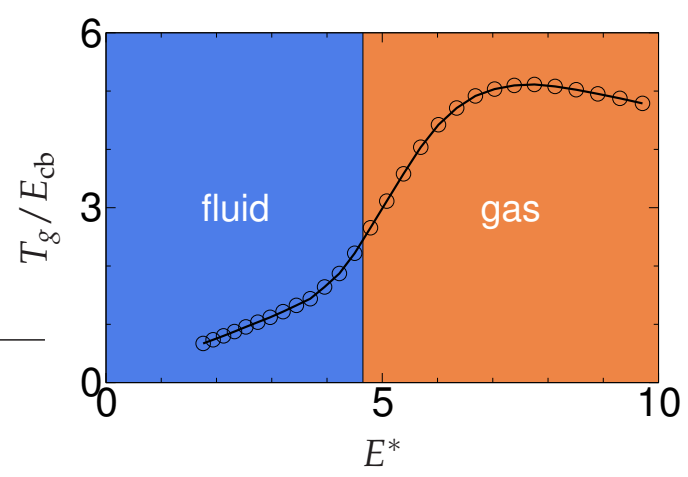

Fig. 3.29 In the case of inelastic collisions the order parameter for the direct transition from a homogeneous fluid to a homogeneous gas state is slightly modified, because there is no jump existing at the transition point any longer. Due to the inelasticity, this phase transition is smeared out compared to the case of elastic collisions and, therefore, the inflection point of the granular temperature, $T_{g} / E_{\mathrm{cb}}$ is used as order parameter in these cases. It is plotted for $\Gamma=2.7$, which is indicated by the white line in the phase diagram of Fig. 3.11.

\section{A.1.3. Solid-gas phase boundary}

The discrimination of solid and gas does not need an extra order parameter and can be done with one of the two order parameters described above.

\section{A.2. Discrimination of inhomogeneous phases}

Inspired by classical theory of simple liquids, where the density has proven to be the appropriate order parameter to detect coexisting phases (see, for instance Yeomans, 1992), some kind of density is also used as an order parameter to detect coexisting phases. As wet granular matter is simulated, the distribution of capillary bridges within the sample is considered, which is called the capillary bridge density, $n_{\mathrm{cb}}(\vec{r})$. Since stationary states are considered, it can be averaged over time. The order parameter is defined similarly as in classical theory as the difference of the capillary bridge density in the two phases. Here, simply the global minimum and maximum value within the system is used as the respective density in the dilute and dense phase. It follows that the order parameter $\Delta n_{\mathrm{cb}}$ is defined as

$$
\Delta n_{\mathrm{cb}}=\max \left(n_{\mathrm{cb}}(\vec{r})\right)-\min \left(n_{\mathrm{cb}}(\vec{r})\right)
$$

where $\vec{r}$ is the lateral position in the D-I dimensional plane, which is projected along the gravity-acting plane. This is shown in Fig. $3 \cdot 30$ for a two-dimensional simulation.

As more simulations are performed, Fig. $3 \cdot 31$ is obtained, which shows the order parameter $\Delta n_{\mathrm{cb}}$ (black curve) for the transition of a homogeneous fluid to a homogeneous gas via a region of fluid-gas coexistence. The difference in the areal density $\Delta n_{\text {area }}$ is, for comparison, also plotted in Fig. $3 \cdot 31$ (red curve). It can clearly be seen ${ }^{10}$ 10. Which is misleadingly claimed differently in (Hager-Fingerle, 2007, pp. 221). 


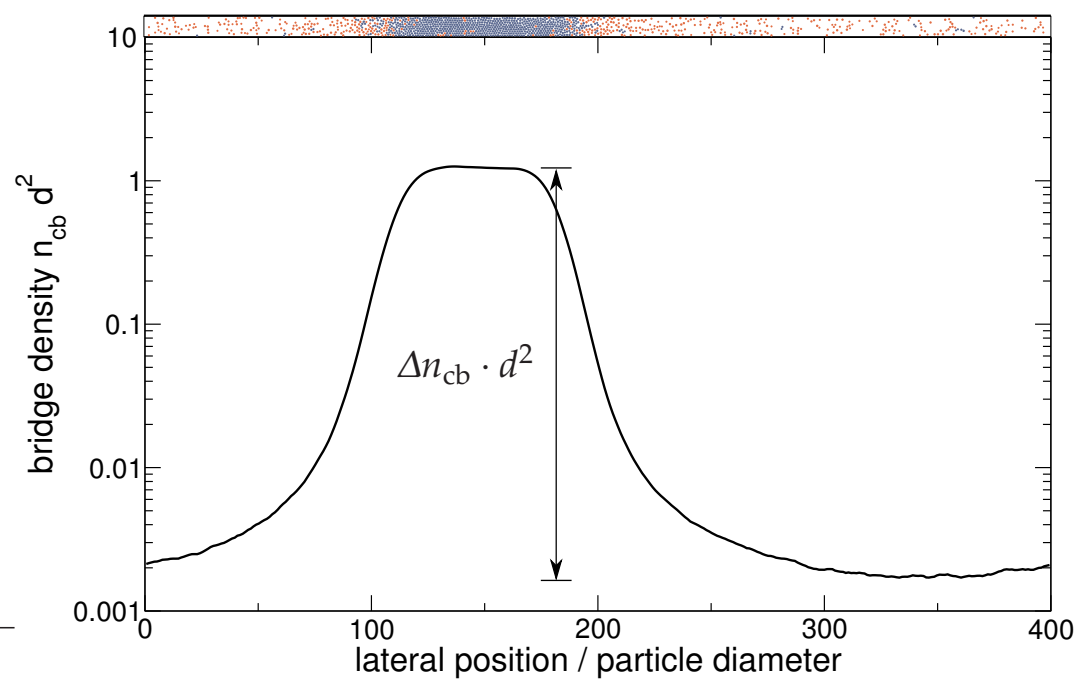

Fig. 3.30 The time averaged capillary bridge density is shown here for a two-dimensional system (which is displayed above the graph) as a function of the (onedimensional) lateral position. The difference in the capillary bridge density, $\Delta n_{\mathrm{cb}}$, is used as order parameter to detect coexisting phases. It is simply calculated as the difference between the maximum an minimum capillary bridge density and normalized by the inverse square of the mean particle diameter, $d$. The driving parameters are $\Gamma=15.0$ and $E^{*}=1.42$.

that the difference in the areal density increases even faster than the difference of the bridge density in the coexisting phase. The reason why the bridge density nevertheless was preferred as order parameter is due to its feature of being closer to zero in the gas phase. Only small fluctuations prevent the difference of the bridge density to be exactly zero in the gas phase.

The steeper increase of the difference in the bridge density is also supported if the critical exponents ${ }^{11}$ for both order parameters are calculated. The fit of the following functions (indicated by dashed lines in Fig. 3.31)

$$
\begin{aligned}
\Delta n_{\mathrm{cb}} & \propto\left(E_{\mathrm{fgc}-\mathrm{g}}^{*}-E^{*}\right)^{\beta_{1}} \\
\Delta n_{\text {area }} & \propto\left(E_{\text {fgc-g }}^{*}-E^{*}\right)^{\beta_{2}}
\end{aligned}
$$

is calculated and leads with $E_{\mathrm{fgc}-\mathrm{g}}^{*}=1.97$ the critical exponents $\beta_{1}$ and $\beta_{2}$ to be

$$
\begin{gathered}
\beta_{1}=0.37 \\
\beta_{2}=0.12
\end{gathered}
$$

Relating the areal density to the capillary bridge density leads to

$$
\Delta n_{\mathrm{cb}} \propto \Delta n_{\text {area }}^{\beta_{1} / \beta_{2}} \approx \Delta n_{\text {area }}^{3} .
$$

11. Critical exponents depend only on a small set of fundamental parameters, like the dimension of the system and are therefore regarded to be universal (Yeomans, 1992, section 2.6.1) and also studied in granular systems (Ristow, 1997). 
Assuming a linear relationship between $\Delta n_{\text {area }}$ and $n_{\text {area }}$ and similarly for $n_{\mathrm{cb}}$ yields

$$
n_{\mathrm{cb}} \propto n_{\mathrm{area}}^{3}
$$

A naive assumption could be that the number of liquid bridges in a unit volume increases quadratically with the number of particles. This is possible if the critical rupture distance is large enough such that all particles can form capillary bridges with all other particles in the unit volume. As the unit volumes are the same for $n_{\mathrm{cb}}$ and $n_{\text {area }}$ this leads to an exponent of two which is reasonably close to the exponent of three found in simulations. Admittedly, this is very crude, as it assumes a critical rupture separation much larger than used in the simulations. Therefore, more work is necessary to derive a more realistic theoretical expression.

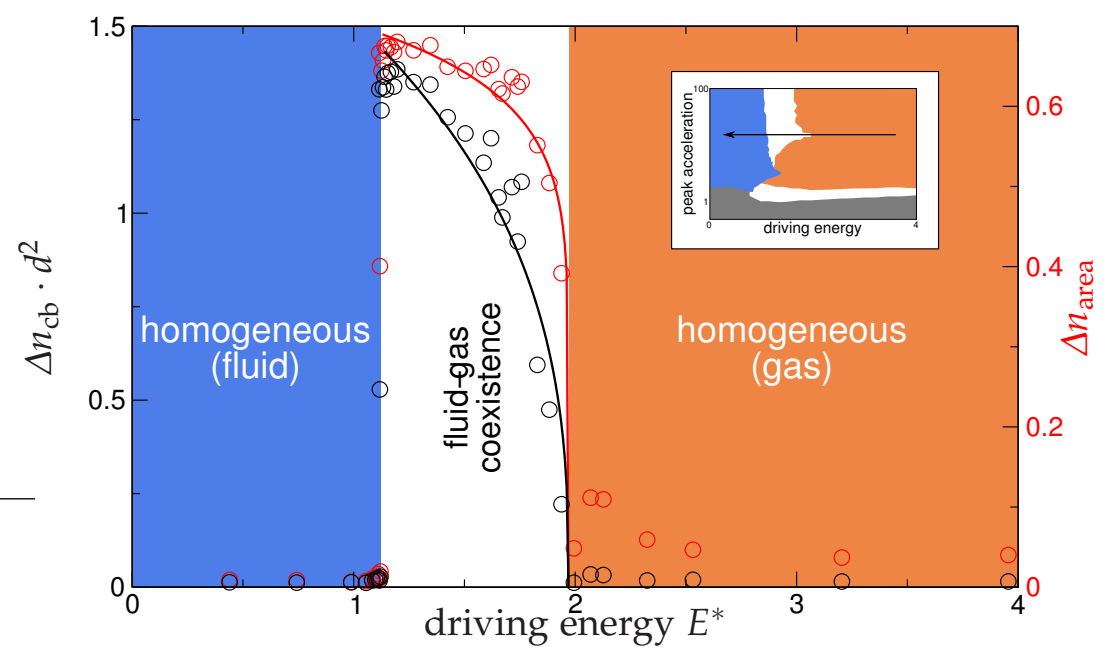

Fig. 3.31 The difference in the bridge density (black) is compared to the difference in the areal density (red), which is used as order parameter to detect the coexisting phase, here between the fluid and gas phase as the driving energy is varied. Simulations (circles) (same system as in Fig. 3.28) are performed along the line which is indicated in the inset. Critical exponents (dashed lines) are detected and for the difference in the bridge density $\beta_{1}=0.37$ and the difference in the areal density $\beta_{2}=0.12$ is obtained. Nevertheless, the difference in the bridge density is used (even if the increase in the difference of the areal density would be much steeper) as order parameter, because the fluctuations, which prevent it from being zero, in the gas phase are much smaller. The peak acceleration of the driving is $\Gamma=15.0$. 


\section{The solid-fluid phase transition and surface melting}

In the previous chapter, the phase diagram of wet granular matter was presented. The present chapter is dedicated to examining the phase diagram obtained by numerical simulation and to studying the solid-fluid phase transition in more detail. A theoretical model is suggested which successfully describes the force-driven solidfluid transition and additionally predicts surface melting to occur at this transition.

\subsection{Introduction}

The solid- to fluid-like (s-f) phase transition in granular matter under vertical vibration is widely studied in the literature of mainly dry granular matter (Melo et al., 1995; Jaeger et al., 1996; Duran, 2000; Kim et al., 2002; Götzendorfer et al., 2005; Umbanhowar and van Hecke, 2005; Huang et al., 2006; Reis et al., 2006; Clerc et al., 2008; Luding, 2009). Understanding the underlying principles which enforce the s-f transition is of great practical interest, for example, in industrial processes where granular matter has to flow through pipes (Duran, 2000). Even in the context of earthquakes the s-f transition plays a role.

It was found in dry granular matter that the s-f transition occurs at a peak acceleration of the driving $\Gamma \approx 1.2$ (Duran, 2000). In the previous chapter, the solidfluid transition in wet granular matter was in the previous chapter also found to be driven by force, however, higher peak accelerations than in the dry case are necessary due to the attractive capillary bridge forces which have to be overcome. In the phase diagram obtained by time-driven molecular dynamics simulations presented in Fig. 3.4(a), it is shown that the s-f transition is mainly driven by force but it will be shown here, that the driving energy, $E^{*}$ seems to act as a second order correction.

\subsection{Theoretical model}

A model is constructed now (see Roeller, 2005) in order to understand the underlying principles involved in the solid-fluid transition. 
To this end, a column of $M$ granular particle layers $h=1, \ldots, M$ is considered, where $h$ is the number of the respective layer starting with $h=1$ directly above the sinusoidally vibrating bottom wall. As the s-f phase transition is mainly driven by the force of the vertical motion of the driving wall, it seems to be a reasonable initial approximation to consider only the forces in the system which act in the vertical direction (perpendicular to the driving wall) in order to obtain a rough prediction for its behavior.

A capillary bridge imposes a capillary force, $F_{\text {capillary, }}$ to the column, which is the same everywhere. It simply reads

$$
F_{\text {capillary }}=F_{B},
$$

where $F_{B}$ is the usual capillary bridge force and is pointing downwards towards the bottom wall. Acting in the same direction is the hydrostatic force, $F_{\text {hydro, }}$ which is caused by gravity. The gravitational force, which is imposed by the $h$ th layer on its subjacent layer, is given by

$$
F_{\text {hydro }}(h)=(M-h+1) m g,
$$

where $m$ is the mass of one particle and $g$ is the acceleration due to gravity. (It is noted that $h=1$ is the lowest height-value which is allowed). Furthermore, the isotropic force caused by the thermal motion of the particles is included. It is approximated by considering the change of momentum per unit time and reads

$$
\begin{aligned}
F_{\text {therm }} & =v p \\
& =\frac{2\left\langle E_{\mathrm{kin}}\right\rangle}{l_{m}} \\
& =\frac{f k_{B} T_{g}}{l_{m}},
\end{aligned}
$$

where $v=v / l_{m}$ is the mean collision frequency, $l_{m}$ is the mean free path, $p=m v$ is the momentum of a particle, $v$ is its velocity, $\left\langle E_{\mathrm{kin}}\right\rangle$ is the mean kinetic energy of the particles, $f$ the number of degrees of freedom, $k_{B}=1$ the 'Boltzmann constant' and $T_{g}=2\left\langle E_{\text {kin }}\right\rangle / k_{B} f$ is the definition of the granular temperature. The bottom wall is sinusoidally vibrating, causing a force, $F_{\text {driv }}$, which reads

$$
F_{\text {driv }}=M m A \omega^{2} \sin (\omega t),
$$

where $A$ is the driving amplitude and $\omega$ is the driving frequency. As the onset of fluidization is studied here, it is reasonable to restrict the consideration to the driving force which reduces the stability the most. This is the case if the driving force is at its maximum value, which is pointing upwards away from the bottom wall. The driving force now reduces to

$$
\max \left(F_{\text {driv }}\right)=M m g \Gamma,
$$


using the usual definition of the peak acceleration $\Gamma=A \omega^{2} / g$. It can be seen that there is no explicit time dependence in the model anymore, such that only stationary quantities have to be considered.

It is now assumed that in a stationary state these four forces have to be balanced, i. e. $F_{\text {capillary }}+F_{\text {hydro }}(h)=F_{\text {therm }}+\max \left(F_{\text {driv }}\right)$, which leads to the equation

$$
\frac{F_{B}}{m g}+1-h=\frac{f k_{B} T_{g}}{m g l_{m}}+M(\Gamma-1)
$$

The dynamics of the system crucially depends on the ratio $l_{m} / s_{\text {crit }}$. As long as $l_{m} / s_{\text {crit }}<1$ is fulfilled in the whole column no bridges are ruptured: hence there is no dissipation and the granular temperature in the column may be assumed to be the same everywhere. The mean free path then is obtained from Eq. (4.8) and reads

$$
l_{m}(h)=\frac{f k_{B} T_{g}}{m g} \frac{1}{\frac{F_{B}}{m g}+M(1-\Gamma)-h+1} .
$$

It can easily be seen that the mean free path increases monotonically as the height, $h$, in the column is increased. This is physically reasonable because the hydrostatic force, which counteracts the constant thermal force, decreases with increasing height.

As the mean free path reaches the order of the rupture distance, $l_{m} / s_{\text {crit }} \approx 1$, the temperature cannot be assumed to be constant in the bulk anymore because dissipation occurs. Setting $l_{m}=s_{\text {crit }}$ and $h=M$ in Eq. (4.8), it is found that as long as $\Gamma<\Gamma_{M}$ with

$$
\Gamma_{M}:=\frac{1}{M}\left(\frac{1}{m g}\left(F_{B}-\frac{f k_{B} T_{g}}{s_{\text {crit }}}\right)+1\right)
$$

the mean free path is smaller than the critical rupture separation, $l_{m}<s_{\text {crit, }}$, up to the topmost layer, $h=M$. The total injected energy travels upwards through the column without rupturing any capillary bridge such that there is no dissipation and which leads to a thermal motion of particles. As soon as $\Gamma \geqslant \Gamma_{M}, l_{m}<s_{\text {crit }}$ is still valid for layers $h \leqslant h_{c}$ with a critical height, $h_{c}$, which is

$$
h_{c}(\Gamma)=M\left(1+\Gamma_{M}-\Gamma\right) .
$$

Through the dissipation, which occurs in layers $h>h_{c}$, the temperature is reduced until $l_{m} \approx s_{\text {crit. }} . \Gamma$ by definition is a positive number so that from the condition $\Gamma_{M}>0$, follows that

$$
T_{g}<\frac{1}{f k_{B}}\left(E_{\mathrm{cb}}+m g s_{\text {crit }}\right) .
$$

It can be observed that $\Gamma_{M}$, above which the topmost layer is fluidized, can even be almost zero if the granular temperature of the granulate is high enough to lift the 
topmost particle by a distance of $s_{\text {crit }}$ against gravity and rupture the (topmost) capillary bridge. The critical acceleration, $\Gamma_{C}$, at which complete fluidization is observed, is obtained by setting $h=1$ in Eq. (4.11). It then reads

$$
\Gamma_{C}=1+\Gamma_{M}-\frac{1}{M}
$$

Using the condition $\Gamma_{M}>0$ again, it is found that

$$
\Gamma_{C}>1-\frac{1}{M}
$$

This is interesting because complete fluidization in this model can already occur at peak accelerations $\Gamma_{C}<1$, depending on the number of particle layers involved, which seems counterintuitive at first glance. However, the necessary energy input to compensate for dissipation is then provided by the kinetic energy of the particle motion rather from the maximum peak acceleration. It can be seen in the following that the granular temperature of the particles can be adjusted independently of the parameter $\Gamma$. The granular temperature of the first particle above the wall, $h=1$, is directly determined by the average kinetic energy obtained through the elastic wall collision. Therefore, it can be written as $T_{g}=\frac{2\left\langle E_{\text {wall }}\right\rangle}{k f}=\frac{E_{\text {kin }}}{k f}$, where $E_{\text {wall }}$ is the time dependent kinetic energy which a particle obtains by a wall collision, $E_{\mathrm{kin}}=m v_{w}^{2} / 2$ is the maximum wall energy and $v_{w}=A \omega$ is the maximum wall velocity. Using this assumption, the critical peak acceleration for complete fluidization reads

$$
\Gamma_{C}=1+\frac{F_{B}}{M m g}\left(1-E^{*}\right),
$$

where $E^{*}$ is the usual driving energy defined as $E^{*}=E_{\mathrm{kin}} / E_{\mathrm{cb}}$. Now it is directly visible that $\Gamma_{C} \leqslant 1$ is obtained for $E^{*} \geqslant 1$ such that the maximum energy injected by a wall collision is sufficient to rupture one capillary bridge.

\subsection{Comparison with simulations}

The theoretical model clearly predicts that interesting features will occur at the solidfluid transition, which will be compared to numerical simulations in this section.

\subsubsection{Scaling of the solid-fluid transition line}

The critical peak acceleration to obtain complete fluidization is predicted by Eq. (4.15). This equation shall be studied, first, as a function of the capillary bridge energy while keeping other parameters constant and, second, as a function of the driving energy again with all other parameters constant. It will be compared to appropriate simulations. 


\subsubsection{Dependence on the capillary bridge force}

In Fig. $4 \cdot 1$ the dependence of $\Gamma_{C}$ on the normalized capillary bridge force, $F_{B} / m g$, as obtained by simulations, is shown, where white filled circles indicate the transition between the solid and the completely fluidized state. The number of particle layers in the simulation is $M \approx 3$. A linear fit according to $\Gamma_{C}=1+F_{B}\left(1-\tilde{E}^{*}\right) / 3 m g$ to the data is shown as solid black line and $\tilde{E}^{*}=0.38 \pm 0.02$ is obtained, which is in good agreement with the driving energies out of the interval $0.35<E^{*}<0.44$ used in simulations. These simulations together with the theoretical model justify the assumption that the solid-fluid transition is intrinsically dependent on the capillary bridge force.

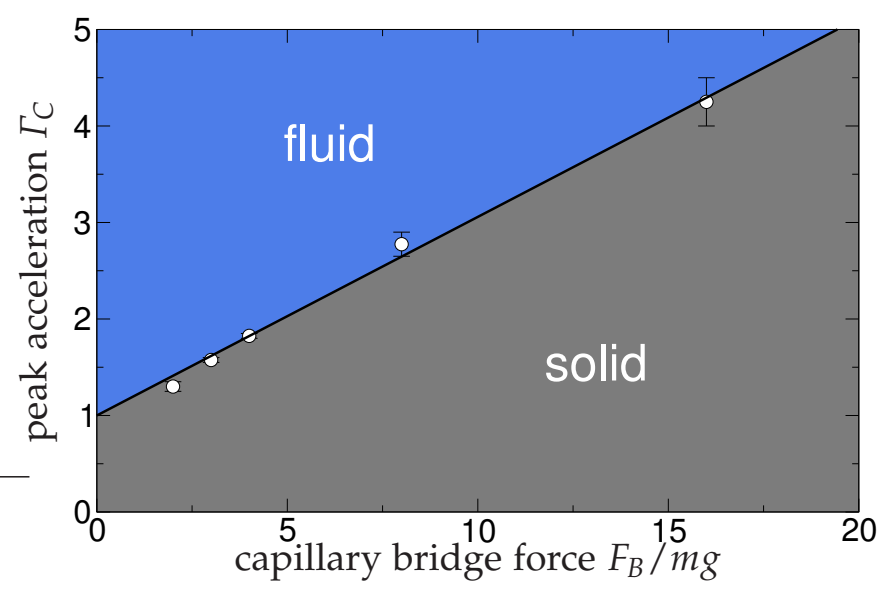

Fig. 4.1 The critical peak acceleration for complete fluidization, $\Gamma_{C}$, for the solid-fluid phase transition, is shown as a function of the normalized capillary bridge force, $F_{B} / m g$. The simulations are performed using the time-driven molecular dynamics simulation method. The solid black line is a fit to the data with $\Gamma_{C}=1+F_{B}\left(1-\tilde{E}^{*}\right) / 3 m g$ leading to $\tilde{E}^{*}=0.38 \pm 0.02$. This is in very good agreement with the driving energy used in the simulations which lies in the interval $0.35<E^{*}<0.44$. The system parameters are the same as in the phase diagram in Fig. 3.3. As before three particle layers are used.

\subsubsection{Dependence on the driving energy}

The red line in Fig. 3.4(a) is the critical peak acceleration $\Gamma_{C}$ for complete fluidization as obtained by Eq. (4.15), which is considered as a function of $E^{*}$ in this case. It has to be compared to the white-filled circles in that figure which denote the solid-fluid transition as obtained from time-driven simulations. The number of layers used in simulations is $M=3$ and the capillary bridge force is $F_{B} / m g=4.0$. The solid to fluid transition as a function of the driving energy, $E^{*}$, as seen in time-driven simulations, therefore is reasonably described by the model. However, deviations from the predictions are visible at $E^{*} \approx 0.75$ in this case, so higher order effects seem to play a role. Even though densely packed particles do not perceive any force in the event-driven model (black squares with white edges in Fig.3.4(a)) the 
simulations are approximately in the same order of magnitude (even if they seem to be independent of $E^{*}$ ) as predicted by the theoretical model.

As the theoretical model does not incorporate a lateral extension it cannot describe the lateral coexistence of solid and gas. Nevertheless, it seems that the transition from solid to the solid-gas coexistence (in the range of $0.8 \lesssim E^{*} \lesssim 1.0$ ) follows approximately the transition line predicted by the model. This suggests that above the transition to the fluid state an other mechanism (which is not included in the model) causes an instability preferring a solid-gas coexistence rather than a homogeneous fluid state. This deserves further investigation.

\subsubsection{Minimum value of the critical peak acceleration}

When one starts to think about phase transitions in granular matter, one is likely to be misled into thinking that the peak acceleration $\Gamma_{C}$ has to be larger than one, such that particles are able to be lift off from their support to start moving. The force balance model derived in this chapter suggests a different behavior. In Fig.4.2, a phase diagram obtained by time-driven simulations is shown with the parameters $F_{B} / m g=4$ and $s_{\text {crit }} / d=0.036$. Note the linear scaling of the axes. It can be seen that the solid to fluid transition in the simulation nicely agrees with the theoretical prediction through Eq. (4.15) (red line). Furthermore, it is obvious that the solid to solid-gas phase transition also occurs at peak accelerations smaller than one. The white dashed line is the theoretical prediction according to Eq. (4.13), which denotes the lowest possible value $\Gamma_{C}$ for the transition to occur. This is in reasonable agreement with the simulations because, as predicted, for $\Gamma<\Gamma_{C}$ only solid states are observed. This was additionally confirmed by event-driven simulations. In order to suppress the lateral inhomogeneity, this should be investigated in more detail in a narrower system as it is likely that the solid-gas coexistence then remains a fluid state.

\subsubsection{Surface melting}

Eq. (4.11) predicts surface melting to occur at the solid-fluid transition. Surface melting is visible in experiments with dry (Warr et al., 1995; Kim et al., 2002; Götzendorfer et al., 2006b) as well as wet granular matter (Huang 2009, priv. comm.). The order parameter to detect the height-dependent fluidization is the diffusion constant and was measured individually in each layer as shown in Fig. $4 \cdot 3$. The layers are defined such that the number of particles is constant in each layer. The order parameter in Fig. $4 \cdot 3$ clearly shows that the top-most layers $(h=6)$ fluidize first at the lowest peak acceleration $\Gamma$ successively followed by the layers below. The simulations are timedriven simulations of six particle layers of three-dimensional, elastically colliding wet granular spheres. In order to suppress lateral phase separation of the solid and fluid phase, as was oberved for the three-dimensional phase diagram in Fig. 3.20, the system is very narrow (only 7.5 particle diameters in each lateral direction). Fig.4.4 


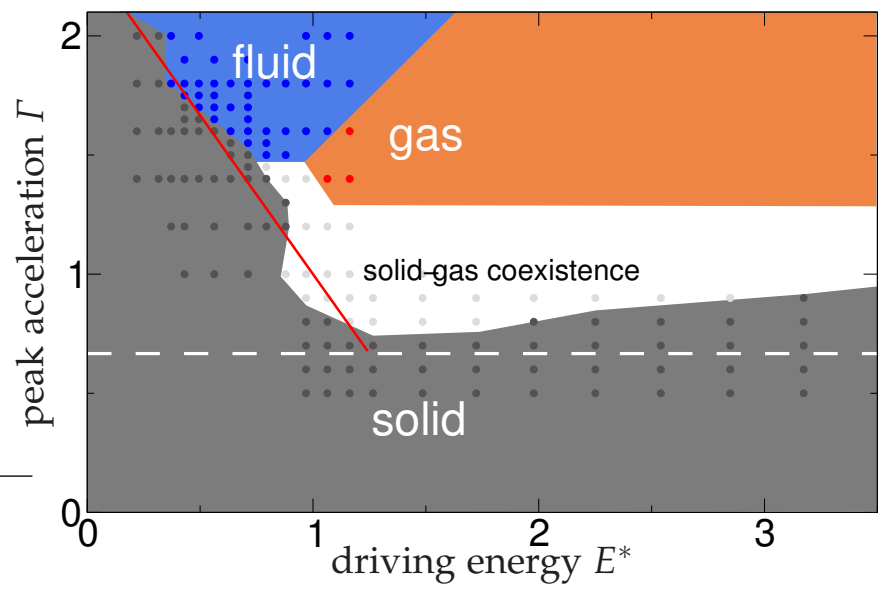

Fig. 4.2 Phase diagram of a time driven simulation of 1200 two-dimensional particles arranged in three layers. Note the linear scaling of the axes. The critical driving acceleration (red line) according to Eq. (4.15) describes well the solid to fluid transition. The white dashed line is the prediction of Eq. (4.13) for the minimum value of the critical peak acceleration to fluidize the granulate which also is nicely satisfied. The parameters for the capillary interaction are $F_{B} / m g=4$ and $s_{\text {crit }} / d=0.036$. All other parameters are the same as in the simulation shown in Fig.3.4(a).

shows as a result the fluidization depth $l_{s}=h_{c}-M$ (circles), which is the number of fluidized layers at the surface, as obtained by simulations in Fig.4.3 as a function of the peak driving acceleration $\Gamma$. It can clearly be seen that the simulation data indeed shows surface melting at the solid-fluid transition as predicted by the theoretical model. However, the functional form given by Eq. (4.11) (red curve in Fig.4.4) deviates significantly from the numerical values. The black line is a fit to the data of the form $l_{s}=l_{0} \ln \left(1 /\left(1-\left(\Gamma-\Gamma_{M}\right) /\left(\Gamma_{C}-\Gamma_{M}\right)\right)\right)$ leading to $l_{0}=1.27$. It suggests that the fluidization depth is diverges logarithmically at the point of complete fluidization. This, surprisingly, is reminiscent of the results obtained for a Landau-type model of surface melting in equilibrium thermodynamics (Lipowsky, 1982; Frenken et al., 1986; Pluis et al., 1990). It is noted in the current work that surface melting was observed in this work only if the polydispersity $\sigma_{p}$ was small enough to allow for a crystallization of the solid phase. However, polydispersity was not changed systematically to verify that surface melting vanishes with increasing polydispersity. This has to be left for future work. Interestingly, the linear dependence of $h_{c}$ predicted by the model is indeed seen in experiments using dry granulates (Kim et al., 2002, Fig. 4(b)). These aspects deserve further investigation.

\subsection{Summary and Outlook}

It was shown in this chapter that a simple model, which assumes the balance of basic forces acting in wet granular matter, leads to a good qualitative agreement with the simulations and thus nicely describes the solid-fluid transition. The critical 


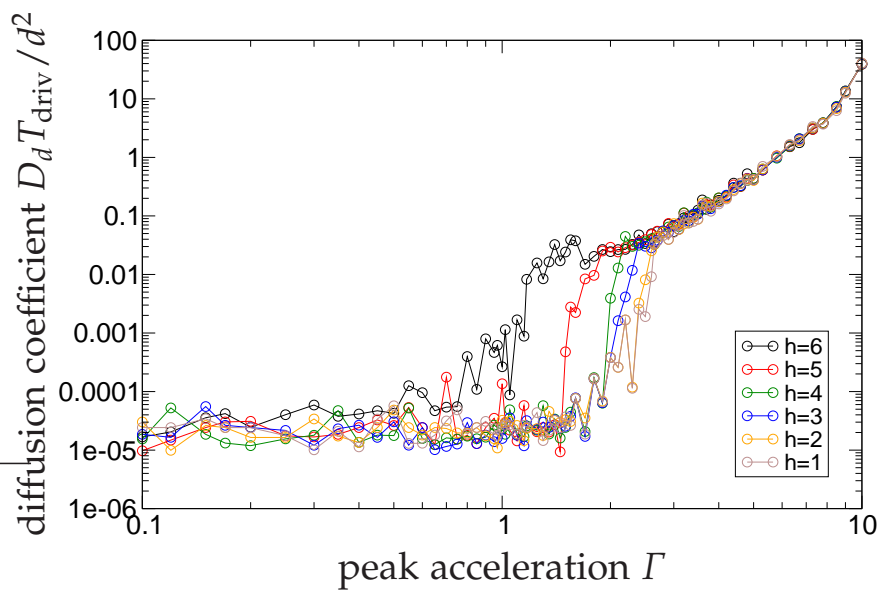

Fig. 4.3 The diffusion coefficient, $D_{d}$, is shown as a function of the peak acceleration, $\Gamma$, and for different heights, $h$, in simulations. The simulation is a time-driven simulation of 300 three-dimensional spheres forming $M=6$ layers and are conducted at constant frequency. The system is very narrow in order to prevent the lateral coexistence instability (system dimensions are $7.5 d \times 7.5 d \times 45 d$ ). The parameters of the capillary interaction are $F_{B} / m g=1$ and $s_{\text {crit }} / d=0.005$. The diagram is out of (Roeller, 2005) were also more details on the order parameter can be found.

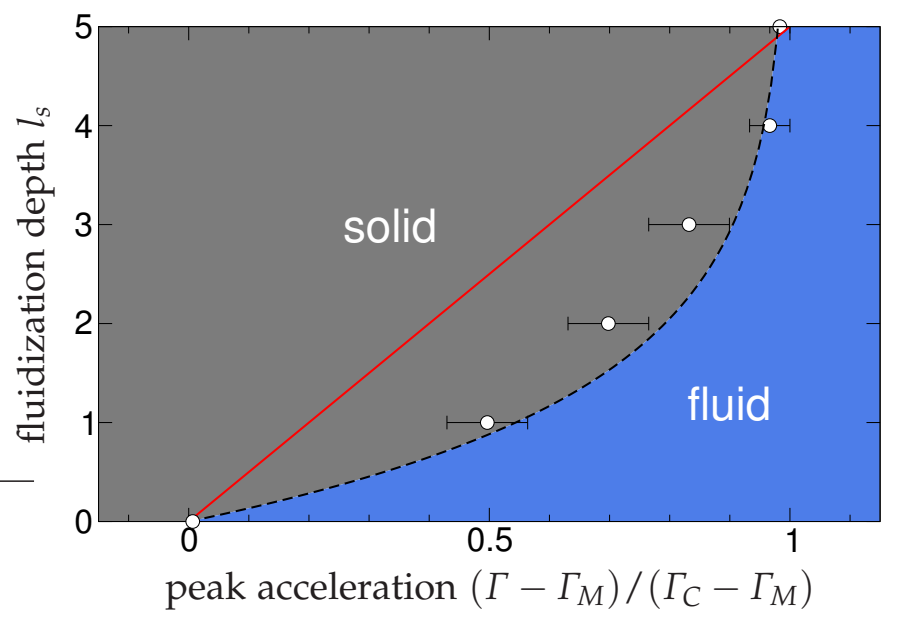

Fig. 4.4 The fluidization depth $l_{s}=h_{c}-M$, which is the number of layers in which the granulate is fluidized, as a function of the normalized peak acceleration. Simulations (circles) reveal surface melting which, however, cannot be quantitatively described by Eq. (4.11) (red line $l_{S}(\Gamma)=$ $(M-1)\left(\Gamma-\Gamma_{M}\right) /\left(\Gamma_{C}-\Gamma_{M}\right)$ with $\Gamma_{M}=1.16$ and $\left.\Gamma_{C}=2.65\right)$. The black line is a fit of the form $l_{s}=l_{0} \ln \left(1 /\left(1-\left(\Gamma-\Gamma_{M}\right) /\left(\Gamma_{C}-\Gamma_{M}\right)\right)\right)$ leading to $l_{0}=1.27$. It suggests that the fluidization depth diverges logarithmically at the point of complete fluidization. The system parameters of the simulation are as in Fig.4.3. 
mechanism for the solid-fluid transition as assumed in the model is the equality of the mean free path between different layers of particle and the critical rupture separation to rupture a capillary bridge and is supported by the simulation results. It is therefore concluded that the solid-fluid transition is in first order force-driven but the driving energy acts as a second-order correction by increasing the thermal energy of the particles. This leads to the interesting result that complete fluidization also can be obtained for peak accelerations lower than one. A lower limit, which depends on the number of particle layers involved, is also provided. This is supported by the simulations. The model also predicts surface melting which can similarly be observed in simulations. The functional form which is predicted to be linear is not found in simulations, instead a logarithmical divergence of the fluidization depth is found. This is reminiscent of the results obtained for a Landau-type model of surface melting in equilibrium thermodynamics (Lipowsky, 1982; Frenken et al., 1986; Pluis et al., 1990). These findings help to understand the nature of the solid-fluid transition and bear reasonable potential in improving industrial processes which deal with this transition. Further work on this topic is desirable to predict also the functional form of the fluidization depth of the surface melting. 


\section{Interfacial energy in wet granular matter}

Two different mechanisms inducing phase transitions (force- and energy-driven) were distinguished in chapter 3 for wet granular matter. In the previous chapter the solid-fluid transition, which is mainly force-driven, was investigated in detail. In this and the following chapter, the coexistence of states occurring at the energydriven phase transition is the object of investigation. In the present chapter a flat and wide system, as before, is used to study the lateral coexistence. The system will be high and narrow in chapter 6, such that the coexistence state occurs in the vertical direction.

After a short introduction, experiments and simulations are discussed which suggest the presence of an interfacial energy in the coexistence region of wet granular matter. The interfacial energy is the driving force to minimize the interfacial area. Thus, in reminiscence of the two-dimensional Ising model, a shape transition will be studied in wet granular matter which is suggested to be a phase-transition-like phenomenon. This is followed by the estimation of the interfacial energy in simulations.

It will further be discussed if an effect similar to interfacial energy can also be found in a two-dimensional system and in the solid-fluid coexistence in the threedimensional simulation. The chapter ends with a short discussion of anomalous diffusion in wet granular matter.

\subsection{Introduction}

Interfacial energy, in liquids equivalently called surface tension, is a physical quantity which is present in everyday life. Just imagine kids playing with soap bubbles and observing the beauty of the emerging interference patterns (Isenberg, 1992). It can be observed in fauna where small insects such as water striders are able to move on the water surface, which resulted in the (more colloquial) name Jesus bugs (Gao and Jiang, 2004). Interfacial energy, however, does not only arise between liquid-gas interfaces but is also present between solid-fluid interfaces and therefore plays a crucial role, for example, in nucleation processes and crystal growth (Tiller, 1991; Pimpinelli and Villain, 1998; Iveson et al., 2001; Hernández-Guzmán 
and Weeks, 2009). Furthermore, not only crystalline growth but "the growth of anything" (Pimpinelli and Villain, 1998) is influenced by interfacial energy and described by the KPZ (Kardar, Parisi and Zhang)-equation (Kardar et al., 1986; Barabási and Stanley, 1995; Pimpinelli and Villain, 1998; Kardar, 2007), which is a generalization of the Burgers (Burgers, 1974) and Edwards-Wilkinson equation (Edwards and Wilkinson, 1982; Hinrichsen, 2000); derived solely from physical and symmetry principles. The basic idea of this equation is the deposition of discrete particles at the surface, which is followed by a rearrangement along the surface. The interfacial energy causes the particles to diffuse along the surface.

Interfacial energy is defined as the energy which is necessary to increase an interface by unit area. Measuring it in a thermodynamically equilibrated system means measuring the excess Gibbs free energy. Even in molecular dynamics simulations this seems a challenging task and is still a topic of ongoing research (Hoyt et al., 2001; Vink and Horbach, 2004; Vink et al., 2005; Laird and Davidchack, 2005; Norizoe et al., 2010). Going to non-equilibrium systems, like granular matter, it becomes even more difficult because it is unclear how interfacial energy should be defined - lacking the concept of free energy (Nagel, 1992). Nevertheless, granular matter is studied in literature making use of the analogy to equilibrium systems by speaking of 'surface tension' (Mehta, 1993; Cheng et al., 2007, 2008). In the present work also the term 'interfacial energy' is used although the author is aware of using it only as an analogy to equilibrium systems.

\subsection{Gas phase growth in fluid-gas coexistence}

A fluid-gas coexistence region was observed at the fluid-gas phase boundary in the phase diagrams presented in chapter 3 . This transition seems to be determined by the balance between the energy injected by the sinusoidal driving and the dissipation by capillary bridges (cf. Sec.3.2). Larger driving energies, $E^{*}$, can lead to an increase of the injected energy. Therefore, it should be possible to rupture more capillary bridges. This suggests that the size of the gas bubble in the fluid-gas coexistence region can be expected to grow with increasing driving energy, $E^{*}$. This behavior was found in previous experimental work on this topic (Kai Huang, priv. comm.) and will be presented here. This will be compared to two- and three-dimensional simulations which show similar behavior. In three-dimensional simulations with periodic boundary conditions, the increasing size of the gas bubble leads to three different shapes of the coexisting phases. Whereof, due to symmetry arguments, only two different shapes have to be considered. The first is a drop shape of the minority phase in the majority phase, for example a small gas bubble in an otherwise fluid system, and the second is a strip state spanning through the system. The transition between these two shapes will then be studied in analogy to a two-dimensional Ising model (Neuhaus and Hager, 2003), where it represents a first order phase transition. 


\subsubsection{Experiments}

Snapshots of an experiment in top view are shown in Fig.5.1. The details of the setup were already given in Sec.3.3.1. In the present experiment the peak acceleration, $\Gamma$, was varied in four discrete steps. The value of the peak acceleration is given by the dashed line and is labeled on the right axis. For each value of the peak acceleration, $\Gamma$, one snapshot which is shown at the bottom of the graph was taken. The time when they were taken is indicated by the arrows in the figure. As the peak acceleration is increased it can be seen in the snapshots at the bottom that the lateral extension of the gas bubble increases. The area of the gas bubble is obtained by image processing and is shown in each snapshot as red overlay. Additionally, the blue circle indicates the size of the gas bubble if a circular shape is assumed. This means that the area of the circle is the same as of the red overlay. The shape of the gas bubble seems to be well approximated by a circular shape. This is only valid as long the gas bubble does not come too close to the side walls of the petri dish. Therefore, the system which is studied is limited to the green circles. The experiment is stopped and repeated when the gas bubble crosses the green line. The area of the gas bubble as obtained by experiments is plotted as a function of time in the main panel of the figure (red circles, labels on the left). Soon after each increase of the peak acceleration of the driving, $\Gamma$, the area of the gas bubble reaches a stationary value. The stationary values are shown in Fig.5.2(a) (green curve) and are normalized by the total area under study. Thus, $a_{g}$ is the fraction of the whole system which is occupied by a gas bubble. The height of the system, $H=6.6 \mathrm{~mm}$, is constant during the experiment and thus it seems reasonable to treat this situation as being quasi two-dimensional. The fraction of the gas bubble, $a_{g}$, is plotted as a function of the normalized and rescaled driving energy $\tilde{E}^{*} \equiv\left(E^{*}-E_{\mathrm{f}-\mathrm{fgc}}^{*}\right) /\left(E_{\mathrm{fgc}-\mathrm{g}}^{*}-E_{\mathrm{f}-\mathrm{fgc}}^{*}\right)$. Here $E_{\mathrm{f}-\mathrm{fgc}}^{*}$ is the critical driving energy for the f-fgc phase boundary (dashed white line) and $E_{\text {fgc-g }}^{*}$ similarly is the critical driving energy for the fgc-g phase boundary (dotted white line) (cf.Sec.3.1.2 for the definitions of the abbreviations). Only a few data points are obtained close to the fgc-g phase boundary. This is because the density difference (cf. Fig. 3.31) between the fluid and gas phase is decreasing close to the fgc-g phase boundary and it becomes more difficult to distinguish the two phases by image processing. The red and the black curves in Fig.5.2(a) correspond to other experiments, for example, with slightly different grain sizes as denoted in the figure caption. For all experiments the filling height $h_{f}$ of the particles in the container was kept constant at approximately $h_{f}=3.6 \mathrm{~mm}$.

The initially expected increase of the gas bubble area $a_{g}$ with increasing driving energy is found in Fig.5.2(a). This is valid for all three different experiments. At the $\mathrm{f}$-fgc transition the gas bubble area $a_{g}$ shows a discontinuous change which gives rise to a hysteresis in the transition, which was also studied but is not presented here. A question which remains open is the origin of the different shapes of the results. 


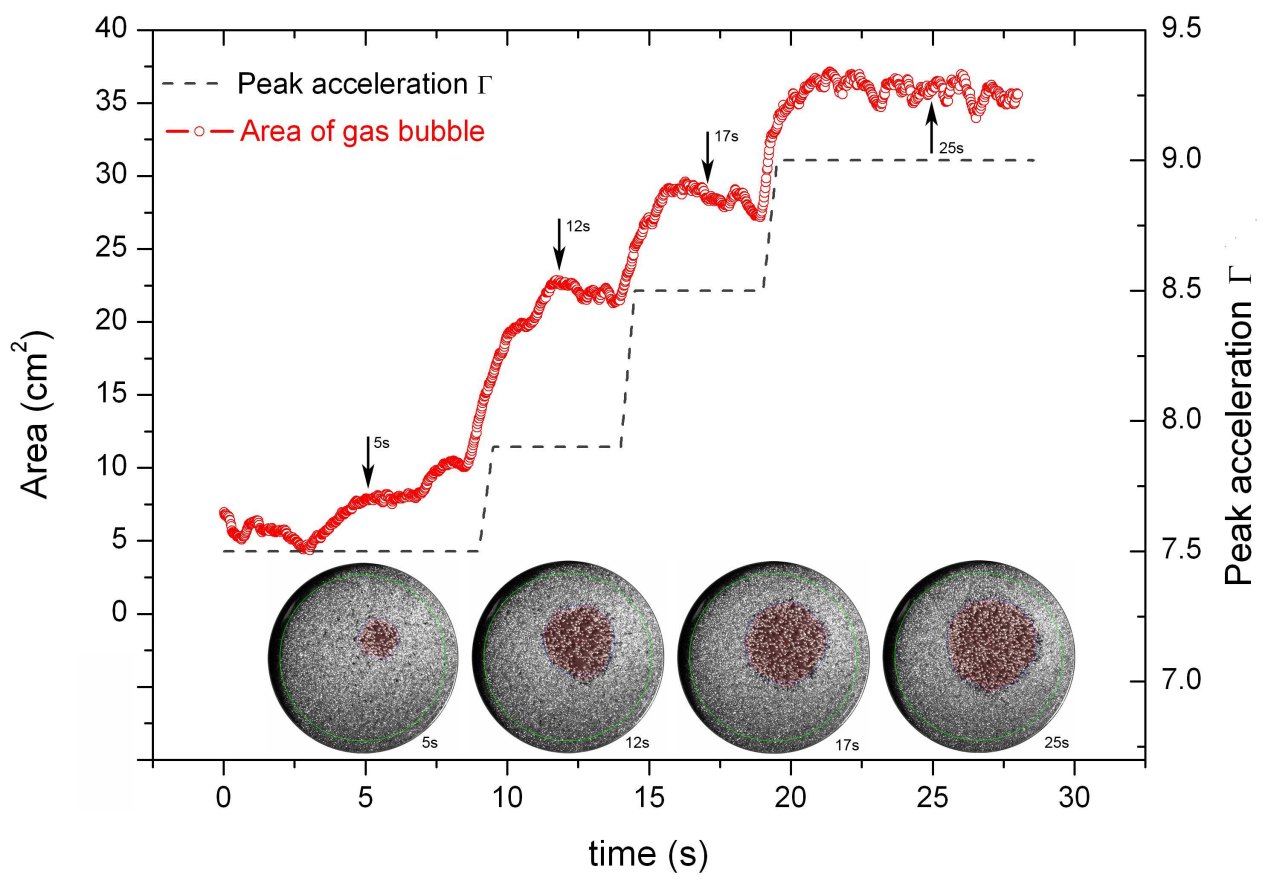

Fig. 5.1 The peak acceleration, $\Gamma$ (dashed line, right vertical axis) is varied in four steps with time. The snapshots clearly show a fluid-gas coexistence state. The increase of the gas bubble fraction for increasing $\Gamma$ is clearly visible. The size of the gas-like region is measured and plotted (red circles, left vertical axis) and also shown as red filled overlay in each snapshot. The average particle diameter is $d=1.5 \mathrm{~mm}$, the driving frequency is constant at $f=50 \mathrm{~Hz}$ and water is used as wetting fluid with a fluid content of $W=2 \%$. The experiments were performed by Kai Huang.

\subsubsection{Simulations}

Two- and three-dimensional simulations were performed in order to study the growth of the gas bubble which was found in experiments. To allow for a comparison between simulations and experiments, the parameters in the simulations were chosen such that they are close to the experimental values. Therefore, the granular particles undergo inelastic collisions with a coefficient of restitution of $\varepsilon=0.8$. The filling height of particles in the system is approximately three particle layers and the height of the system is 9 and 8 particles in two and three dimensions, respectively. This leads to a relative filling ratio (defined as the number of particle layers divided by the height of the system) of 0.33 in two and 0.42 in three-dimensions. In the experiment this value was 0.55 and thus indicates a significantly denser filling compared to the simulations. Therefore, deviations between simulation and experiment are expected. Other system parameters are noted in the caption of Fig. 5.2(b).

The fraction of the system, $a_{g}$, which is occupied with a gas bubble is plotted in Fig. 5.2(b) for the two-dimensional system. The solid black line is a fit to the data of the form $a_{g}=0.72\left(\tilde{E}^{*}\right)^{\beta_{c}}$ with an exponent of $\beta_{c}=0.32 \pm 0.02$ indicating a con- 
(b) Simulation (2D)

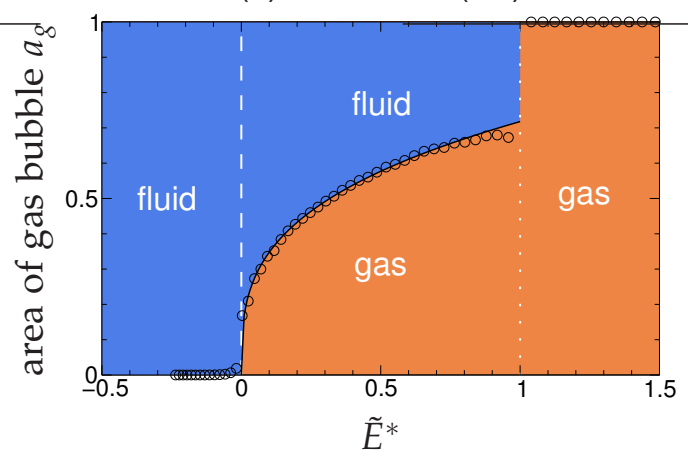

(a) Experiment

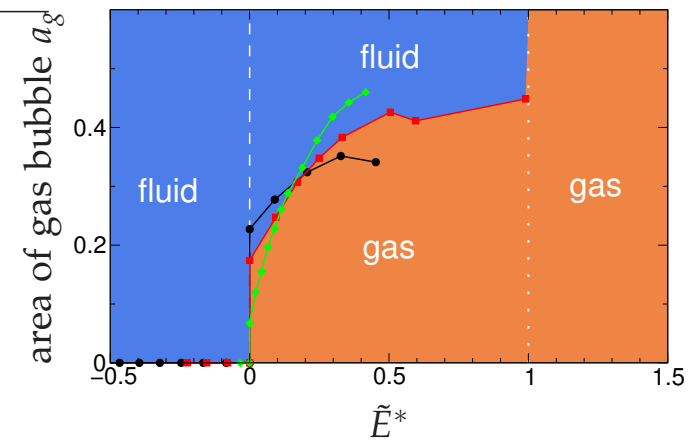

(c) Simulation (3D)

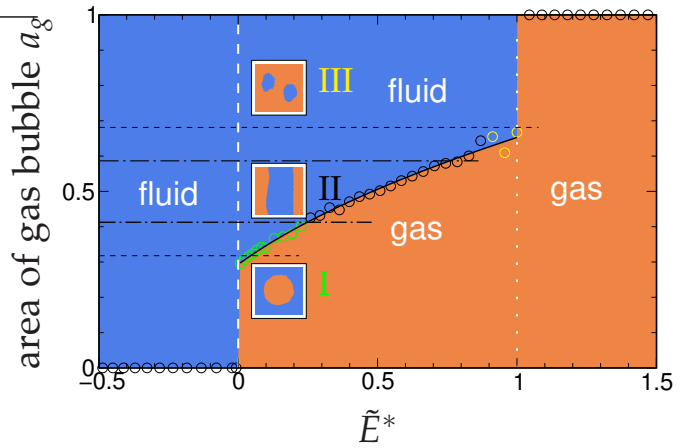

Fig. 5.2 The area fraction of the total system which is occupied by the gas bubble, $a_{g}$, is shown for an experiment (a), a two-dimensional simulation (b) and a three-dimensional simulation (c). It is shown as a function of the normalized driving energy $\tilde{E}^{*} \equiv\left(E^{*}-E_{\mathrm{f}-\mathrm{fg}}^{*}\right) /\left(E_{\mathrm{fgc}-\mathrm{g}}^{*}-E_{\mathrm{fffc}}^{*}\right)$. The two-dimensional simulations (b) reveal a continuous change at the f-fgc phase boundary $\left(\tilde{E}^{*}=0\right)$. In contrast, a discontinuous transition is obtained in the three-dimensional system, which is in qualitative agreement with the (three-dimensional) experiments. The exponent in subfigure (b) is obtained from a fit (solid black line) of the form $a_{g}=0.72\left(\tilde{E}^{*}\right)^{\beta_{c}}$ as $\beta_{c}=0.32 \pm 0.02$. The insets in subfigure (c) show the shape of the coexisting state in the top view of the three-dimensional system, where blue indicates the fluid and orange the gas phase. Three different shapes can be distinguished: a circular-shape of the gas bubble (I), a strip-shape (II), and circular-shape of the fluid bubble (III). They correspond to driving energies as follows: $\tilde{E}_{1}^{*}=0.02$ (I), $\tilde{E}_{\| I}^{*}=0.66$ (II) and $\tilde{E}_{\| I}^{*}=0.99$ (III). The dashed black lines in subfigure (c) indicate the regions up to which the respective shape is theoretically favorable in the sense of minimal interfacial area. The dash-dotted lines denote the boundary as obtained by simulations up to which metastable shapes can be observe. It is discussed further in the text. The experiment was performed (by Kai Huang) at constant frequency $50 \mathrm{~Hz}$ (black curve) and $100 \mathrm{~Hz}$ (red and green curve). The particle diameters are $1 \mathrm{~mm}$ (black), $1.2 \mathrm{~mm}$ (red) and $1.5 \mathrm{~mm}$ (green) and the liquid content of the wetting liquid in the container is $W=2 \%$. The parameters used in the two-dimensional simulation are identical to the one used in Fig.3.11. For the three-dimensional simulation $1.2 \times 10^{5}$ polydisperse particles with polydispersity $\sigma_{p}=0.06$ are used, which are confined to a box of size $8 d \times 192.5 d \times 192.5 d$. The critical rupture separation is $s_{\text {crit }} / d=0.0625$, the bridge energy is $E_{\mathrm{cb}} / m g s_{\text {crit }}=4.0$, the coefficient of restitution between particles is $\varepsilon=0.8$. The peak acceleration used in simulations is $\Gamma=100$. 
tinuous change of $a_{g}$ at the $\mathrm{f}-\mathrm{fgc}$ transition ${ }^{1}$. A qualitative change of this functional dependence is observed in the three-dimensional simulation and can be seen in Fig.5.2(c) (circles). Here, a discontinuous change of $a_{g}$ is found at the f-fgc transition, which is in qualitative agreement with the experiment. However, no quantitative agreement between simulations and experiment can be found. The experimental values are in all cases lower than the corresponding values in the three-dimensional simulation. This means that the gas bubble in the experiment is smaller than in the simulations. A possible explanation for this could be the higher relative filling height, which results in a higher mean particle density. It is well known that the dissipation of kinetic energy in wet granular matter in the dense fluid regions is much larger than in a dilute gas (Hager-Fingerle, 2007). At the same time the energy injection is large in the low density areas. If the mean particle density is now increased this leads to a decrease of injected energy and to an increase of dissipated energy and thus reduces the overall kinetic energy in the system and thus less particles seem to be driven into a gas-like state. It is easily possible to study both, experiments and simulations, at different filling heights such that this argument can easily be checked.

After studying the size of the gas bubble the focus is now shifted to the resulting shape of the coexisting phases. In the experiment, the shape of the bubble was found to be approximately circular as long as it does not come into contact with the wall. In two-dimensional simulations, the shape in the flat system is almost trivial if, as done in the following, the influence of gravitational forces are neglected. Then it can be approximated as a simple plug of varying size. Thus, the much more interesting system is the three-dimensional system where three different shapes can be identified in the simulations and which is studied here. The three small insets in Fig.5.2(c) schematically show these different final shapes of the coexisting phases, where blue indicates the fluid and orange the gas-like region. The first one shows a small gas bubble in an otherwise fluid system (I). The second one shows a strip shape, where each phase spans through the whole system via the periodic boundary conditions $^{2}$ (II). The third one shows two fluid droplets in an otherwise gaseous system (III). This system was not yet in its final stationary state as there is usually a single droplet configuration found. The different shapes correspond to different driving energies as follows: $\tilde{E}_{\mathrm{I}}^{*}=0.02, \tilde{E}_{\mathrm{II}}^{*}=0.66$ and $\tilde{E}_{\mathrm{III}}^{*}=0.99$. The colors of the circles in (c) indicate the shapes which are found in the respective simulation (green circles: I, black circles: II and yellow circles: III). For each of these three shapes the corresponding snapshot of the system is displayed in Fig. $5 \cdot 3$.

1. Note that $a_{g}$ is largely independent of the peak acceleration of the driving, $\Gamma$, as was revealed by two-dimensional simulations which are not shown here. Basically, it only depends on the driving energy $\tilde{E}^{*}$. This seems reasonable as it was assumed that the driving energy determines the quantity of particles in the gas bubble.

2. The periodic boundary conditions in both lateral directions means that the particles move along the surface of a torus. This geometry causes the strip shape to be stable. If the system, for example, were on the surface of a sphere, then this strip shape would be an unstable shape. 


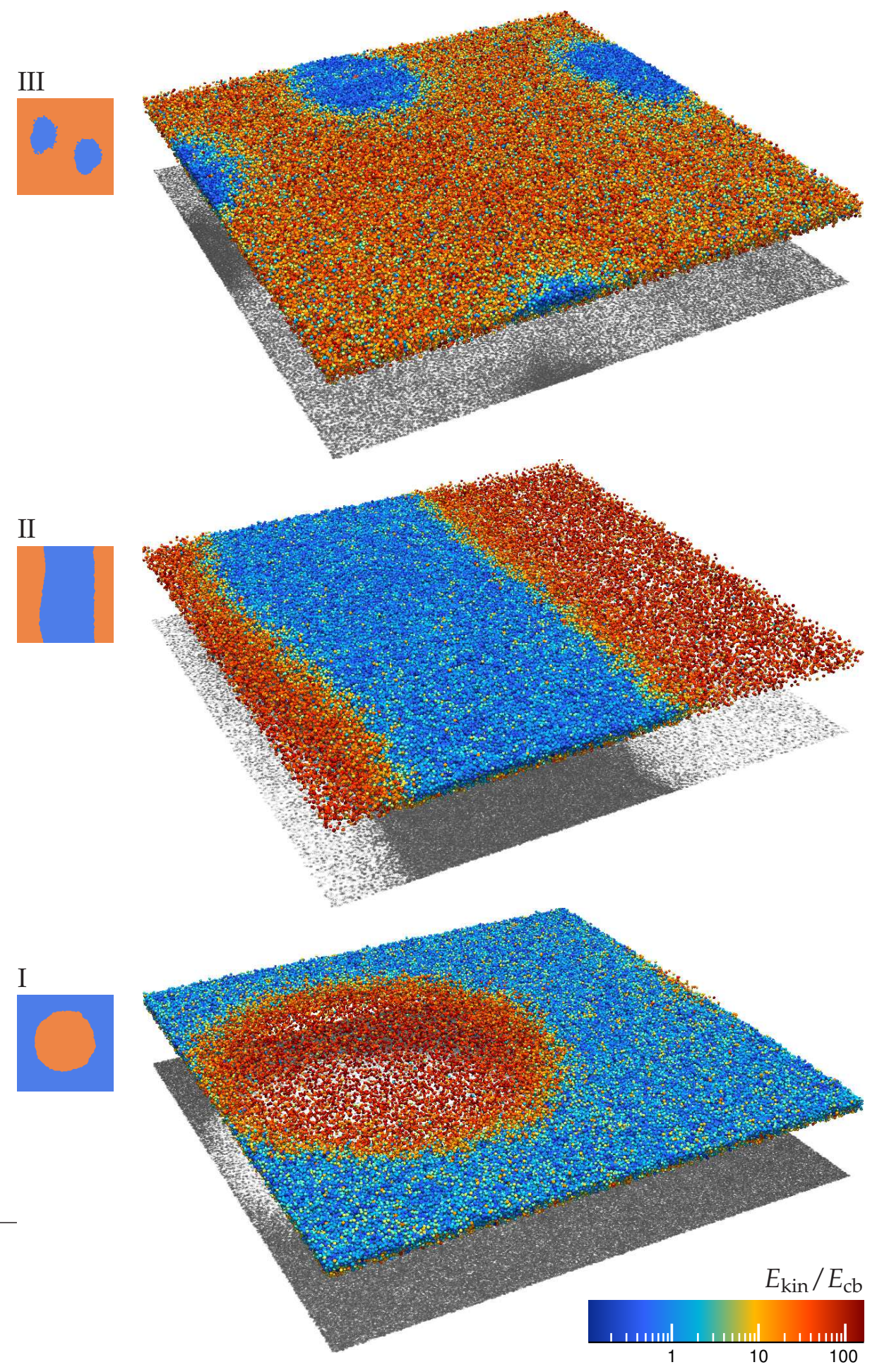

Fig. 5.3 Three snapshots of simulations are displayed which show the three different shapes of the coexisting phases. A circular-shape of the gas bubble (I), strip-shape (II) and circular-shape of the fluid droplet (III). They correspond to the driving energies $\tilde{E}_{I}^{*}=0.02$ (I), $\tilde{E}_{\mathrm{II}}^{*}=0.66$ (II) and $\tilde{E}_{\mathrm{III}}^{*}=0.99$ (III). The other system and driving parameters of the simulations are noted in the caption of Fig.5.2. 
The thermodynamically preferred shape is determined by minimal total free energy. Based on that argument a simple theoretical model is reviewed here, which was shown to predict a transition between a droplet and a strip shape in a coexistence region of the two-dimensional Ising model (Leung and Zia, 1990; Neuhaus and Hager, 2003). In this system's equilibrium state, the transition between the drop to the strip shape was shown to be a first order phase transition. The theoretical model involves, besides an isotropic surface tension, only geometrical arguments for the switching between the drop and the strip shape. The method involves two steps. First, the equilibrium shape of the coexisting phases is calculated based on the minimization of free energy. It will depend only on the area fraction $a_{g}$ and determines the transition point between the drop and the strip shape. The second step is the calculation of the saddle point configuration. This determines the height of the energy barrier which is involved in this first order phase transition. Because the calculation involves the minimization of the free energy in the system it is not directly applicable to the system of wet granular matter. Simply because there is obviously nothing like a free energy known for the system of wet granular matter. However, the calculation is based mainly on geometrical arguments of how to minimize the interfacial area at the transition between the two states. These geometrical arguments seem to bear reasonable potential to be applicable also at the transition from the circular to the strip shape in wet granular matter.

The equilibrium shape which is obtained in such a system in thermal equilibrium with periodic boundary conditions in both directions is either a circle or a strip shape. At the transition point between these two shapes the interfacial length of the circle has to be the same as for the strip shape. This immediately leads to the radius of the droplet $r=L / \pi$ at this transition, where $L=L_{x}=L_{y}$ is the lateral extension of the square-based system. The relative area fraction of the system which is then occupied by the droplet is given by $a_{g}=1 / \pi$ and determines the transition point in-between the two shapes. This is the first result and shall now be compared to the simulations. Two different shapes occur in simulations which are reminiscent of a droplet shape. This is shape (I), where a gas bubble appears in an otherwise fluid system and the shape (III), where a fluid droplet appears in an otherwise gas-like system. For the argument of minimizing the free energy only the shape and the length of the interface are important. It particularly does not include any distinction of the two phases which are in contact. Therefore, the shape (I) is equivalent to the shape (III) in this argument such that these two situations can be assumed to be a droplet of the minority phase which is immersed in the majority phase. Due to this symmetry argument, the region where the strip shape is favored by energy minimization is found to be in the interval $1 / \pi \leqslant a_{g} \leqslant 1-1 / \pi$. These limits are given by the dashed black lines in Fig.5.2(c). There it can be seen that a gas bubble shape (I) is still found in the region where the strip shape thermodynamically is already preferred. Thus, the transition points as predicted by theory are in qualitative agreement with the simulations but no quantitative agreement is found.

To further improve the agreement between the simulation and the theoretical pre- 
diction it is turned back to the second step of the theoretical argument in the equilibrium situation. As the transition between the circular and the strip shape was found to be a first order phase transition, this gives rise to an energy barrier which has to be overcome. Thus, for example, a metastable droplet shape is characterized by a droplet radius which is larger than the critical radius, $r>L / \pi$. This immediately brings to mind the question about the 'saddle point' configuration. The 'saddle point' configuration is the geometric shape with the lowest interfacial length that can lead without any further increase of the free energy to both: the transition to the drop shape as well as to the strip shape. This is sketched in Fig.5.4. The additional length (compared to the equilibrium state) which is associated with this 'saddle point' configuration determines the height of the energy barrier. It was conjectured in (Leung
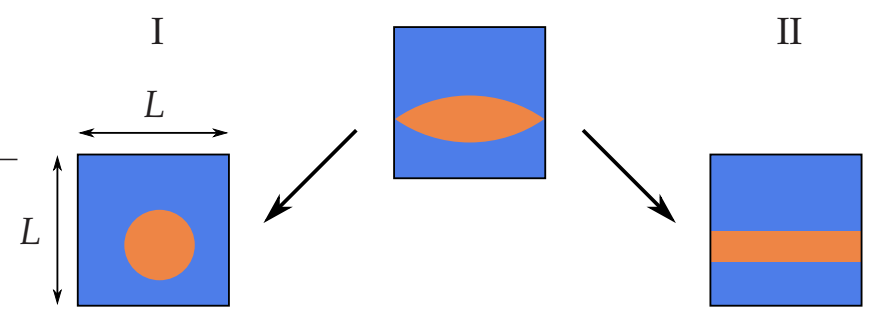

Fig. 5.4 A droplet shape is sketched in (I) in a system of width and height $L$, while the strip shape is shown in (II). The saddle point configuration is shown in between the two images. The area of the orange gas bubble shall be the same in all three sketches. The saddle point configuration is composed of two lens-shaped arcs and minimizes the excess interfacial length for the given area. Being in the saddle point configuration means that the interfacial area is reduced by both, going to a drop or strip state.

and Zia, 1990) that the shape of the 'saddle point' configuration is a lens-shaped 'pinched strip'. This led to the result that the percentile increase of the excess length of the 'saddle point' configuration is given by

$$
\frac{1}{2} \partial a_{D / S}-1=0.1346 \ldots
$$

where $\partial a_{D / S}$ is the non-dimensional interfacial length of the lens-shaped 'pinched strip' at the saddle point. This result was obtained by only assuming the geometrical shape of the saddle point configuration and thus seems to bear reasonable potential to be transferable to other systems as already discussed in (Leung and Zia, 1990). In the simulations shown in Fig.5.2(c), a drop shape (I) was observed in the regime where the strip shape (II) is already preferred by minimization of the interfacial length. This seems to give rise to an excess length of the droplet compared to the strip shape. This droplet can be considered to be reminiscent of a 'metastable' state. The maximum excess length which is obtained in the simulations will be estimated below.

The transition from the droplet shape (I) to the strip shape (II) as found in simulations is marked by the dash-dotted line in Fig.5.2(c). The transition occurs at 
a critical area of the gas bubble, $a_{D / S} \approx 0.413 \pm 0.013$, which is estimated as the arithmetic mean of the value above and below the boundary. This is now assumed to approximately determine the saddle point (neglecting for instance the influence of possible fluctuations). Out of this area the excess length can be estimated by assuming a droplet of mean radius $\tilde{r}=\sqrt{a_{D / S} / \pi}$, where $\tilde{r}=r / L$ is the normalized radius. Then the excess length is obtained to be $\frac{1}{2} \partial a_{D / S}-1=\sqrt{\pi a_{g}}-1$ and approximately determines the percentile increase of the excess length of the 'saddle point' configuration. With the value for $a_{D / S}$ obtained above, this becomes

$$
\frac{1}{2} \partial a_{D / S}-1=0.139 \pm 0.018
$$

This estimate compares nicely with the theoretical prediction for the equilibrium model given by Eq.5.1. However, the good agreement of the numerical values should be taken with great care. (If the analogy to the equilibrium system is taken seriously then small fluctuations seemingly can trigger the transition between the two states and the value for the excess length should decrease with increasing time). Thus this result is considered to be a qualitative agreement. More important than the apparent agreement of the numerical values, is that this result suggests the presence of a quantity similar to an 'interfacial energy' in this system of wet granular matter. The corresponding value for the transition from regime (III) to (II) cannot be obtained due to the large scattering of the data in that region. Therefore, symmetrically the same value as was obtained for the transition from (I) to (II) is marked and only serves as a guide to the eye.

One question immediately arises with regard to the data presented in Fig.5.2(c). Why do only the gas bubble shapes (I) appear as metastable states in region (II), but no metastable strip states (II) appear in the region (I)? An possible explanation for this is given by looking at the preparation of the initial state of the simulation. At the beginning of each simulation, the particles are distributed on a regular lattice and obey a Gaussian velocity distribution of mean granular temperature $T_{g} / E_{\mathrm{cb}}=40.0$. No capillary bridges are present between the particles. Then, at the very beginning of each simulation the particles are freely cooling for a short period of time. The time evolution of the system which then follows can be seen in the snapshots shown in Fig. $5 \cdot 5$ (which shows a simulation of elastically colliding particles). Thus, an initial nucleus seems to form somewhere in the system and then expands to its stationary size. This is qualitatively similar for the case of inelastically colliding particles, even though the number of initial nuclei can be larger than one ${ }^{3}$. As all simulations seem to start with a gas bubble which is expanding, this readily explains why in the transition from (I) to (II) only metastable droplet states are observed. At the same time this assumption suggests a possible explanation for the deviations at the transition from the strip (II) to the fluid drop shape (III). However, the data shows considerably

3. At driving energies close to $\tilde{E}^{*}=0$ a single nucleus is formed within the system. By successively increasing $\tilde{E}^{*}$ the number in initial nuclei is growing. 
more noise 4 at the fgc-g transition, which makes it difficult to quantitatively examine the behavior. Nevertheless, this suggests that simply by preparing the initial state in a specific manner enables the study of the transition in the favored direction. The comparison between simulations and experiments suggests a dependence of the size of the gas bubble on the packing fraction of particles in the system. This can easily be utilized to test the universality of the value provided for the percentile increase of the excess length of the 'saddle point' configuration in Eq. (5.2).

In the next section simulations are shown which try to estimate the value of the effective 'interfacial energy' which was suggested to be present.

\subsection{Interfacial energy}

In this section an experiment is shown which also suggests the presence of a quantity like an interfacial energy in wet granular matter. It is followed by the estimation of the interfacial energy in simulations, which is done by applying a sinusoidal force to the strip state and measure the resulting deformation. The resulting gain in potential energy of the deformation is assumed to be balanced by the loss of energy due to the increased interfacial area. At the end of the section a dynamical method is presented which attempts to estimate the mobility of the particles in the fluid phase.

\subsubsection{Observation}

Snapshots of an experiment are shown in Fig. 5.6 for different times. The system is a flat petri dish which is viewed from above and contains water-wetted glass spheres. In contrast to the experimental snapshots shown in the previous section, the driving does not change with time, but is kept constant at the values given in the caption. Two gas bubbles were formed initially in Fig.5.6(a) and start merging in Fig.5.6(b), forming a cusp where the two gas bubbles start touching. This cusp is rounded in Fig.5.6(c) until only a single, larger gas bubble remains in Fig.5.6(d). This strongly suggests the presence of an interfacial energy also in experiments.

\subsubsection{Estimation of the interfacial energy in simulations}

Several techniques can be thought of where the equilibrium formalism can be technically applied to the present system of wet granular matter. The two most promising are, for example, the monitoring of interfacial fluctuations (Hoyt et al., 2001) and the

\footnotetext{
4. A short comment shall be made concerning the increasing noise which occurs in the data in Fig. 5.2(c) at the fgc-f transition. It is partially caused by the difficulty to precisely detect the fluid phase at this transition because the density difference between the fluid and the gas state decreases. This can be seen, for example, by comparing the shadows of Fig.5·3(III) with (II), where the density of the gas-like region in (III) is significantly higher compared to the one in (II). Additionally, the interface has a certain width and the precise definition slightly influences the value which is obtained for $a_{g}$. The largest influence is expected where the density difference is small.
} 


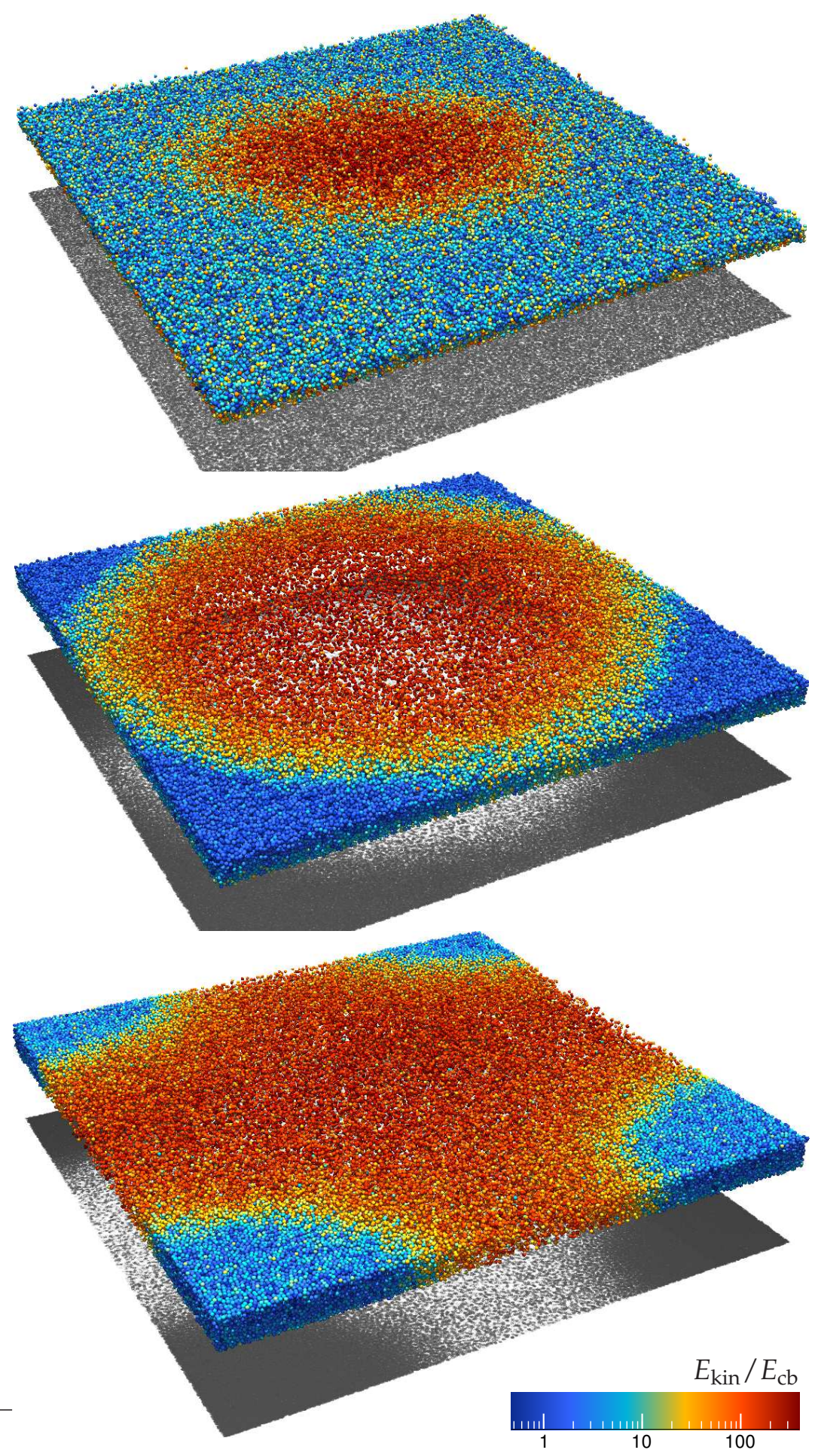

Fig. 5.5 Snapshots which are taken equidistantly in time (starting at the top) of an event driven molecular dynamics simulation of $1.1 \cdot 10^{5}$ wet spheres using the thin-thread model with perfectly elastic collisions between particles. The gas bubble (top) expands until it reaches system size (middle) and the diamond shaped fluid phase (middle) forms a circular spot located at the system corner. The particles are slightly polydisperse with $\sigma_{p}=0.06$ and mean diameter $d=1$. The system is $10 d \times 175 d \times 175 d$ particle diameters large, the rupture distance is $s_{\text {crit }} / d=0.07$, the capillary bridge energy is $E_{\mathrm{cb}} / m g s_{\mathrm{crit}}=3.5$, the mass of one particle is $m=1$ and the acceleration due to gravity is $g=1$. The particles were initially distributed homogeneously on a regular lattice within the system and the mean granular temperature was $T_{g} / E_{\mathrm{cb}}=20.0$ with a gaussian velocity distribution. 

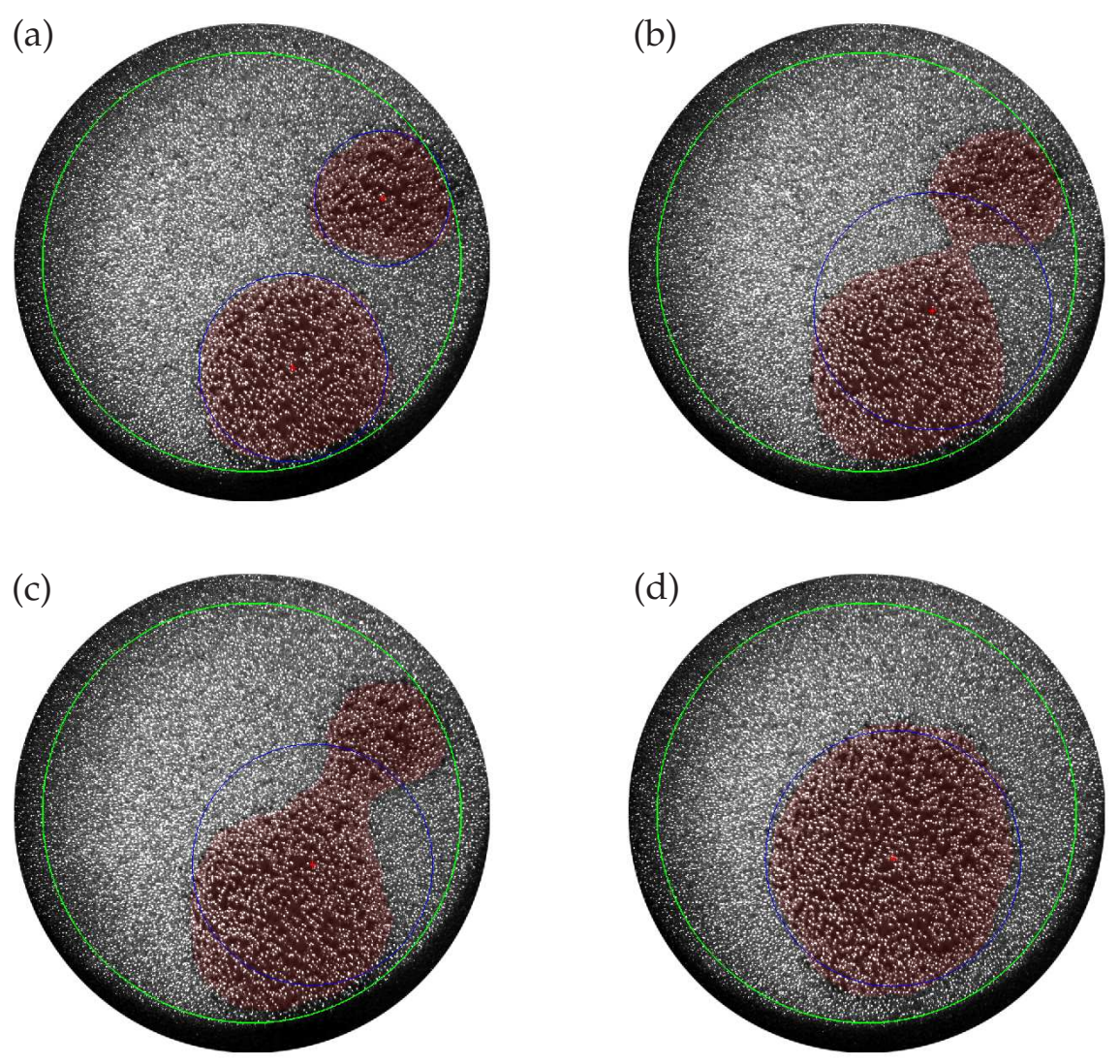

Fig. 5.6 Snapshots of an experiment with water-wetted glass spheres of diameter $1.06 \mathrm{~mm}$ confined in a flat petri dish $(9 \mathrm{~mm})$ and viewed from above. Two gas bubbles are formed initially (a) and start merging in snapshot (b) forming a cusp where the two gas bubbles touch. This cusp is rounded (c) until only one gas bubble remains (d). This suggests a interfacial energy which is causing this rounding process. The particles are filled up to a height of $3 \mathrm{~mm}$ and the liquid content is $W=1 \%$. The driving frequency is $50 \mathrm{~Hz}$ and the peak acceleration is $\Gamma=9.5$. The snapshots are not taken equidistantly in time. Experiment performed by Kai Huang. 
deformation of the strip shape with an applied shear force. The latter one shall be applied here. A simple possibility for such a force, which has to be applied perpendicular to the strip, is a force sinusoidally varying in space. The acceleration field of this shear force, which was introduced in Sec.2.4.4 is given by

$$
a_{d}(\tilde{x})=-a_{\mathrm{smax}} \cos (2 \pi \tilde{x}),
$$

where $a_{\text {smax }}$ is the maximum acceleration of the external shear force field and $\tilde{x}=$ $x / L_{x}$ is the normalized length. The deformation of the interface in the stationary state is given by

$$
\tilde{f}_{h}(\tilde{x})=\tilde{A}_{d} \cos (2 \pi \tilde{x}),
$$

where $\tilde{f}_{h}=f_{h} / L_{x}$ is the normalized position of the interface and $\tilde{A}_{d}=A_{d} / L_{x}$ is the normalized deformation amplitude. For the slightly deformed interface $\tilde{f}_{h}(\tilde{x})$ the gain in potential energy is given by

$$
H_{V}=\rho \int_{0}^{1} \mathrm{~d} \tilde{x} \tilde{f}_{h}(\tilde{x}) a_{d}(\tilde{x}),
$$

where $\rho=m N_{p} / 1$ is the mass density, $m$ the mass of one particle and $N_{p}$ is the number of particles in the fluid phase. This gives for the interface described by Eq. (5.4)

$$
H_{V}=\frac{1}{2} N_{p} F_{\mathrm{smax}} \tilde{A}_{d}
$$

where $F_{\text {smax }}$ is the maximum of the applied force. In the stationary state this gain in energy $H_{V}$, must be balanced by the loss in energy, $H_{\sigma}$, caused by the increase of the interfacial area. Assuming a continuum model, $H_{\sigma}$ is approximated for smallcurved, $D-1$ dimensional surfaces in a $D$-dimensional system and interfacial en$\operatorname{ergy} \sigma$ by

$$
H_{\sigma}=\sigma \int \mathrm{d}^{D-1} \tilde{x}\left[\sqrt{1+\left(\nabla \tilde{f}_{h}\right)^{2}}-1\right] \approx \frac{\sigma}{2} \int \mathrm{d}^{D-1} \tilde{x}\left(\nabla f_{h}\right)^{2},
$$

where the integration limits are such that the whole interface is taken into account. After short calculation the energy necessary to deform the interface in a threedimensional system is found to be

$$
H_{\sigma} \approx 2 \pi^{2} \sigma \tilde{A}_{d}^{2}
$$

The factor of two results from the two interfaces in the simulation (red lines in Fig. (5.7). In the stationary state, where the deformation amplitude, $\tilde{A}_{d}$, is constant, there must be $H_{\sigma}=H_{V}$. This means that the gain in potential energy induced by the sinusoidal shear force (Eq. (5.6)) has to be balanced by the increased interfacial 
energy (Eq. (5.8)). If the deformation amplitude, $\tilde{A}_{d}$, and the number of particles, $N_{p}$, in the fluid phase are known for a given shear force, $F_{\text {smax }}$, the interfacial energy can finally be approximated as

$$
\sigma=\frac{N_{p} F_{\text {smax }}}{(2 \pi)^{2} \tilde{A}_{d}}
$$

Using this approximation, the interfacial energy shall now be estimated in eventdriven simulations. The thin thread model is used to account for the capillary interaction while particle collisions occur perfectly elastic. The diagram in Fig. 5.7 shows the (time-averaged) capillary bridge density as a grey shade, where white means no bridges (gas state) and black means many bridges (fluid state). It is plotted against the two lateral coordinates and averaged over the height of the system. The cosine-shaped shear force is visualized above the diagram. A deformation of the fluid phase is observed which follows the applied shear force. Thus, the resulting deformation can be well approximated by a cosine-shaped function as indicated by the red lines, which are fits to the interfaces and lead to a deformation amplitude of $A_{d} / L_{y}=0.066$. The simulation here is performed in a square base simulation box with $L=192.5 d$. A snapshot of the system is shown in the inset of the figure.

In Fig. 5.8 the time evolution of the deformation amplitude $A_{d}$ is shown for two different shear forces, $F_{\text {smax }}$. The initial state of the simulation is a system with a strip state spanned straight through the system. The system which was used in the simulation is in a stationary state before the shear forces are applied to it. It can be seen that the deformation amplitude is increasing until it reaches a stationary state and then only fluctuates around the stationary value. The deformation, $A_{d}$, is larger if the shear force, $F_{\mathrm{smax}}$, is larger (red curve in Fig. 5.8 ). It seems as if the potential energy gained through the deformation can indeed be balanced by an interfacial energy.

The resulting deformation amplitude, $A_{d}$, is plotted in Fig.5.9 as function of the applied shear force, $F_{\text {smax }}$. Approximately, a linear dependence can be found, which is expected according to Eq. (5.9) if the interfacial energy is constant for different shear forces. The independence of the interfacial energy on the applied shear force is is a striking result in itself. This suggests that the interfacial energy can be well approximated with this simple approach reflected by Eq. (5.9), even though the system under study is far from equilibrium. As the driving energy, $\tilde{E}^{*}$, is increased, the slopes of the curves in Fig.5.9 are increasing, which means that the interfacial energy is decreasing. It is found in simulations (Fig.5.10 (left)) that the fraction of particles which are in the fluid phase stays in good approximation constant, even though the shape is changing through the different values of the applied shear force. The number of particles in the fluid phase mainly depends on the driving energy, $\tilde{E}^{*}$, as it can be seen in in the figure on the right hand side, and decreases with increasing driving energy.

Using the slope obtained in Fig. 5.9 and the number of particles in the fluid phase as obtained in Fig. 5.10, the interfacial energy can now be estimated by using Eq. (5.9). 


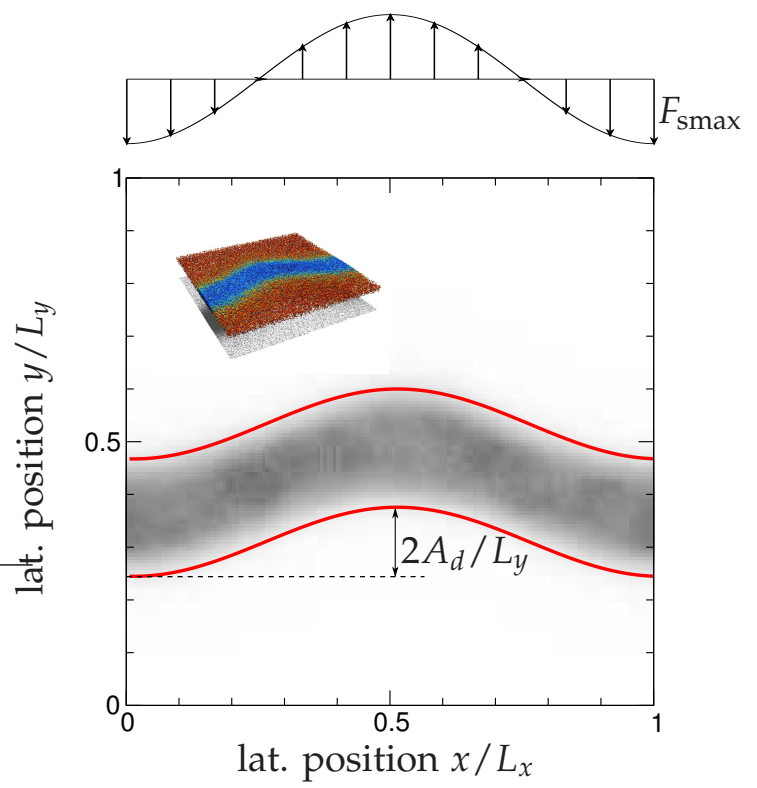

Fig. 5.7 Capillary bridge density is shown as gray scale (dark: many bridges, white: no bridges) in lateral spatial resolution (averaged in vertical direction and in over 50 snapshots in time). The red curve is a cosine fit to the phase boundary which determines the deformation amplitude, $A_{d} / L_{y}=$ 0.066 , and shows that the deformation follows the applied shear force (shown above the diagram with maximum shear force $F_{\text {smax }}$ ). The inset shows a snapshot of the three-dimensional simulation of $1.2 \times$ $10^{5}$ elastically colliding particles confined in a box of dimensions $8 d \times 192.5 d \times 192.5 d$ obeying periodic boundary conditions. The driving parameters of the shaking are $\Gamma=100.0$ and $E^{*}=2.14$, while the maximum shearing force is $F_{\mathrm{smax}} L_{x} / E_{\mathrm{cb}}=1.04$. The capillary bridge energy is $E_{\mathrm{cb}} / m g s_{\mathrm{crit}}=4.0$ at a critical rupture separation of $s_{\text {crit }} / d=0.0625$.

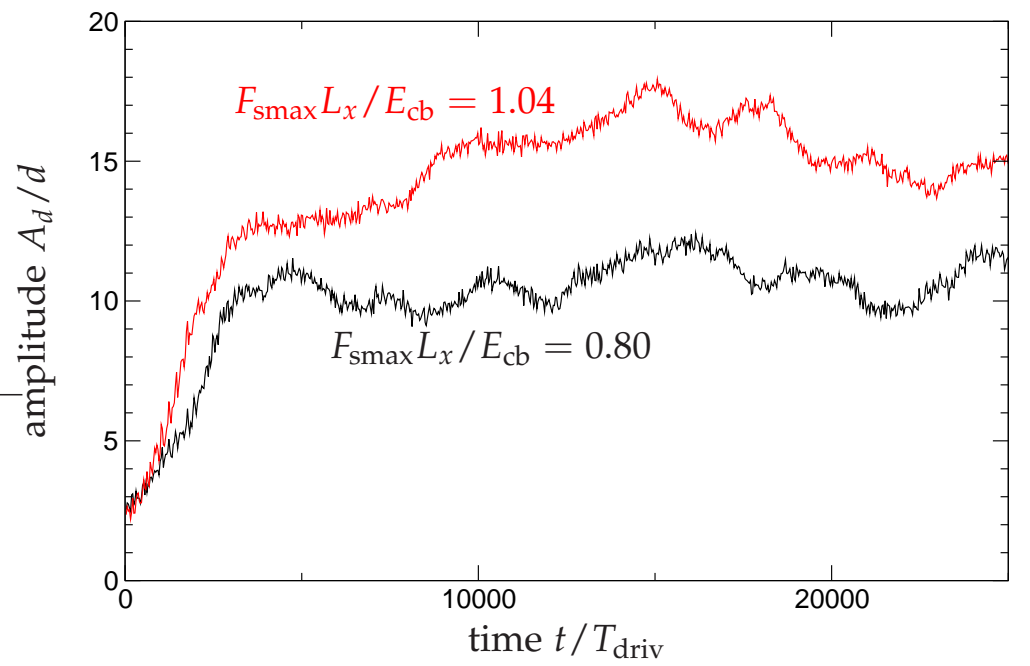

Fig. 5.8 The time dependence of the deformation amplitude, $A_{d} / d$, of the sinusoidally deformed fluid strip is shown for different shearing forces. A stationary state is reached in both cases even though larger shearing forces lead to larger deformations. 


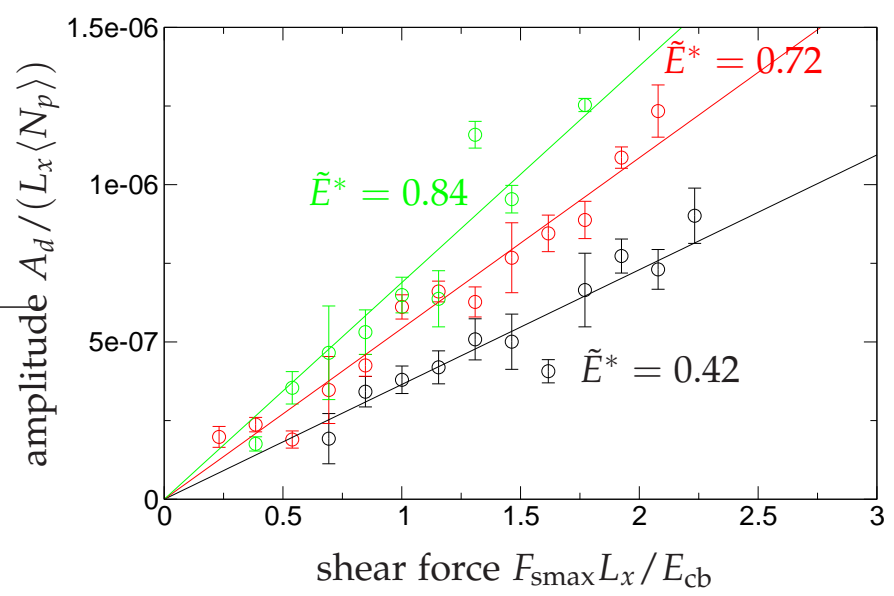

Fig. 5.9 The deformation amplitude, $A_{d}$, as obtained by three-dimensional simulations of elastically colliding spheres is plotted as a function of the maximum shearing force, $F_{\text {smax }}$. It is normalized by the length of the system, $L_{x}$, and the average number of particles in the fluid phase, $\left\langle N_{p}\right\rangle$. The approximate linear behavior suggests that Eq. (5.9) is a reasonable estimate of the energies involved in the transition . The different slopes for different driving energies $\tilde{E}^{*}$ denote a dependence of the interfacial energy on the driving energy. Simulation parameters are as denoted, and otherwise the same as in Fig.5.7.
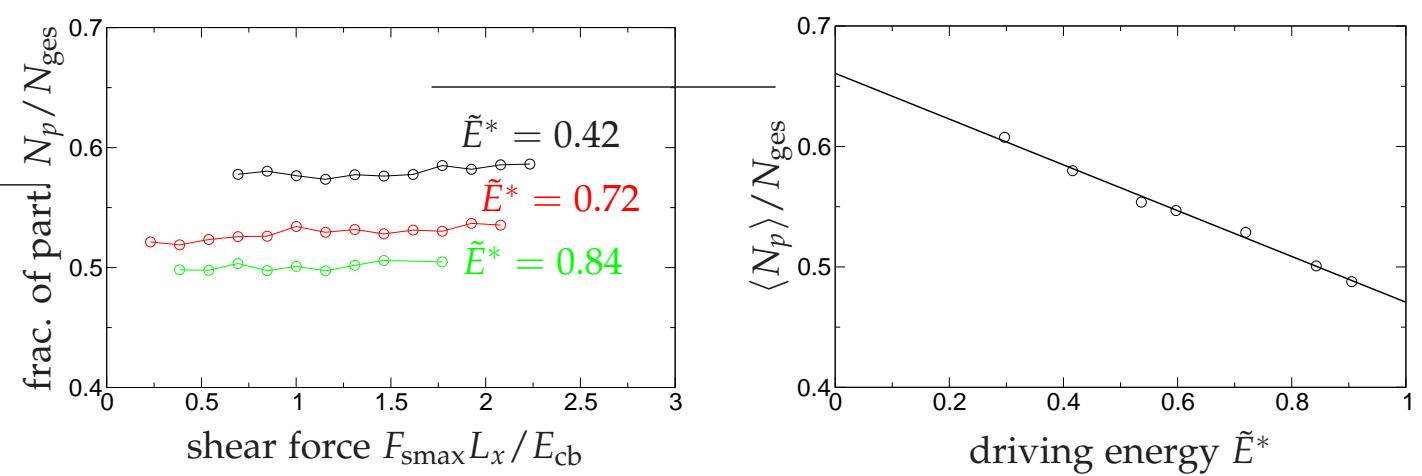

Fig. 5.10 The fraction of particles which are in the fluid phase is shown as a function of the maximum shear force, $F_{\mathrm{smax}} L_{x} / E_{\mathrm{cb}}$, (left figure), where a slight increase can be observed for larger shear forces. However, it is mainly dependent on the driving energy $\tilde{E}^{*} \equiv\left(E^{*}-E_{\mathrm{f} f \mathrm{fgc}}^{*}\right) /\left(E_{\mathrm{fgc}-\mathrm{g}}^{*}-E_{\mathrm{f}-\mathrm{fgc}}^{*}\right)$ (right figure). The simulation parameters are as denoted or otherwise the same as in Fig.5.7. 
The final result is shown in Fig. $5 \cdot 11$ (black) and shows the interfacial energy, $\sigma$, as a function of the driving energy, $\tilde{E}^{*}$. The interfacial energy is normalized by the surface tension of the wetting liquid $\gamma$, because the capillary bridge force is well approximated by Eq. (2.8) as $F_{B} \approx \pi d$ in the case of complete wetting. It is obtained that the interfacial energy, $\sigma$, lies between $15 \%$ and $40 \%$ of the surface tension value of the wetting liquid.

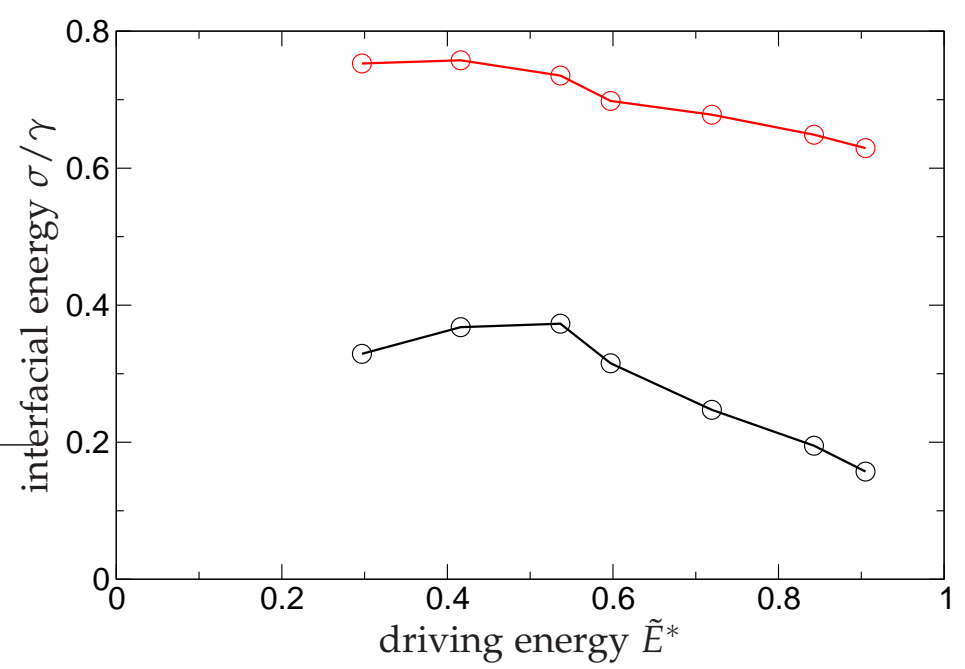

Fig. 5.11 The interfacial energy, $\sigma$, between a fluid and gas phase as obtained by three-dimensional simulations (black) of elastically colliding hard spheres and theoretical estimation by Eq. (5.10) (red) is shown at different driving energies, $\tilde{E}^{*}$, of the sinusoidal shaking. It is normalized by the surface tension $\gamma$ of the wetting liquid (cf. Eq. (2.8)), while the driving energy is normalized such that the fluidgas coexistence region is projected to the normal interval between zero and one by $\tilde{E}^{*}=\left(E^{*}-\right.$ $\left.E_{\mathrm{f}-\mathrm{fgc}}^{*}\right) /\left(E_{\mathrm{fgc}-\mathrm{g}}^{*}-E_{\mathrm{f}-\mathrm{fgc}}^{*}\right)$. Simulation parameters as denoted and otherwise the same as in Fig.5.7.

In the following a short estimate of the interfacial energy shall be given. Therefore, the interfacial energy is estimated by the evaporation of a liquid droplet assuming that the energy of evaporation is known. To this end the fluid particles are microscopically assumed to be ordered on a regular three-dimensional lattice and attracting each other. A sketch of this situation is shown for a two-dimensional lattice of size $L$ in Fig. 5.12. The average distance between the sites is assumed to be $\bar{d}$. The surface area which is now created by the evaporation of the fluid can be estimated by cutting the lattice into its smallest pieces. Every cut which is made through the three-dimensional lattice creates a surface area of $2 L^{2}$. Four of these cuts are marked as red lines in the sketch. For complete evaporation of the fluid, in each dimension $L / \bar{d}$ of these cuts have to be made. Thus, the total surface area created by complete evaporation is $A_{\text {tot }}=6 L^{3} / \bar{d}$. For a given energy of evaporation, $\Delta H$, the surface tension then can finally be estimated as $\sigma=\Delta H / A_{\text {tot }}$. The energy to evaporate all granular fluid particles is simply given by the number of capillary bridges in the volume times the capillary bridge energy and thus reads $\Delta H=n_{\mathrm{cb}} L^{3} E_{\mathrm{cb}}$. The capillary bridge force $F_{B}=E_{\mathrm{cb}} / s_{\text {crit }}$ is given by $F \approx \pi d \gamma$, where $\gamma$ is the surface 
tension of the wetting liquid. The mean distance between particles is estimated as $\bar{d}=1 / n^{1 / 3}$, where $n$ is the particle number density. Finally the interfacial energy of the wet granulate can be estimated by

$$
\frac{\sigma}{\gamma} \approx \frac{\pi}{6} n_{\mathrm{cb}} d s_{\mathrm{crit}}\left(\frac{1}{n}\right)^{1 / 3}
$$

In order to compare this result with the simulations, the capillary bridge density and the particle number density of the fluid state are used as obained from the simulations. The resulting interfacial energy for wet granular matter in the system under study is plotted in Fig.5.11(red) as a function of the normalized driving energy. This simple estimate seems to be of the same order of magnitude as the values obtained in simulations (black). Quantitative deviations seem reasonable as the packing of particles is not as regular as assumed by the simple estimate. A qualitative agreement is found for the decrease of the interfacial energy with increasing driving energy. In the simple estimate, it is caused by the decrease of the capillary bridge density with increasing driving energy. Therefore, this strongly suggests that the interfacial energy in this system of wet granular matter originates from the capillary bridges.

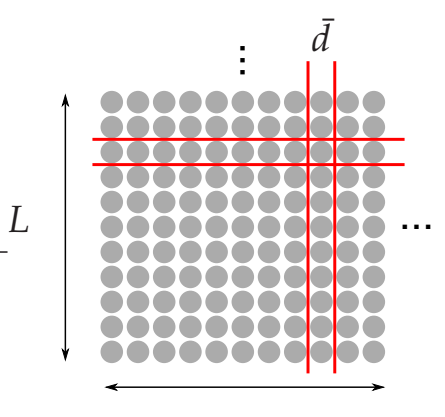

$L$

Fig. 5.12 Left: A sketch of a liquid droplet of size L. Each cut through the lattice creates additional surfaces. The distance between the lattice sites is $\bar{d}$.

The sinusoidal deformation of the interface which is obtained through the applied shear force, immediately raises the question of what happens if the external force is switched off. If there is an interfacial energy in the system the expectation is that the strip dynamically relaxes back to its 'equilibrium' shape. Equilibrium in this context simply means the state with minimal interfacial area which is a strip shape with a flat interface. This dynamical behavior is studied in the next section.

\subsubsection{Estimation of particle mobility}

The dynamical relaxation of a sinusoidally deformed interface is discussed in this section. The expected result is an exponential decay of the deformation amplitude with time. This will then be compared to the numerical simulations. 
The dynamical behavior of a fluid interface is governed by the minimization of the interfacial area. The corresponding Langevin equation for a thin liquid film, where gravitational forces are neglected, is

$$
\frac{\partial \tilde{f}_{h}(\tilde{x}, t)}{\partial t}=\sigma \mu_{f} \nabla^{2} \tilde{f}_{h}(\tilde{x}, t)+\chi(\tilde{x}, t),
$$

where $\sigma$ is the interfacial energy, $\mu_{f}$ is the effective mobility of the fluid particles and the noise $\chi(x, t)$ has the correlations $\left\langle\chi(x, t) \chi\left(x^{\prime}, t^{\prime}\right)\right\rangle=2 \gamma_{s} \delta\left(x-x^{\prime}\right) \delta\left(t-t^{\prime}\right)$, where $\gamma_{s}$ is the strength of the noise. The units of $\sigma \times \mu_{f}$ are the same as for a diffusion coefficient. For a derivation of how the interfacial energy in the Langevin equation is connected with a diffusion-like term the reader is referred to (Kardar, 2007). The Langevin equation is linear and solvable by Fourier transforms (Kardar, 2007). The initial deformation of the interface is given by

$$
\tilde{f}_{h}(\tilde{x}, t=0)=\tilde{A}_{d} \cos (2 \pi \tilde{x}),
$$

where $\tilde{A}_{d}(t)=A_{d}(t) / L_{x}$ is the normalized amplitude and $\tilde{A}_{e_{0}} \equiv A_{d}(t=0)$ is the deformation at start. Eq. (5.11) is solved for the average deformation amplitude by averaging over several realizations and thus neglecting the noise. The time-evolution of the average deformation amplitude with the initial conditions given by Eq. (5.12) leads to

$$
\tilde{A}_{d}(t)=\tilde{A}_{e_{0}} \exp \left(-(2 \pi)^{2} \sigma \mu_{f} t\right)
$$

which is an exponential decay of the deformation amplitude with time. As it was first shown in (Edwards and Wilkinson, 1982) the Langevin equation displayed in Eq.5.11 is similarly valid for a coarse grained system. The exponential decay shall now be studied in numerical simulations of the wet granular system.

When performing simulations, the initial configuration used is the final stationary state obtained by the sinusoidal deformation of section 5.3.2. For the sake of computational efficiency, a different program was used where the sinusoidal shearing was not implemented. This, however, led to the fact that the capillary bridges were not included in the initialization. Thus at the beginning of each simulation, the capillary bridges have to be formed by collisions. A typical outcome of such a relaxation process is shown in the snapshots in Fig.5.13. The initially deformed strip (top) decreased its deformation (middle) until it recovered a flat interface again (bottom).

Fig. 5.14 shows the time evolution of the deformation amplitude which is averaged over 20 different simulations on a semi-logarithmic scale. The error bars denote standard deviation. It can be seen that the deformation amplitude decays with time. At the very beginning slight deviations from the exponential decay can be observed. This is most likely caused by the absence of capillary bridges at the beginning of each simulation. This is followed by a region where an exponential decay seems to occur, as suggested by the solid straight lines, which are fits to the corresponding curve. However this is less than a decade and thus is not sufficient to suggest an 

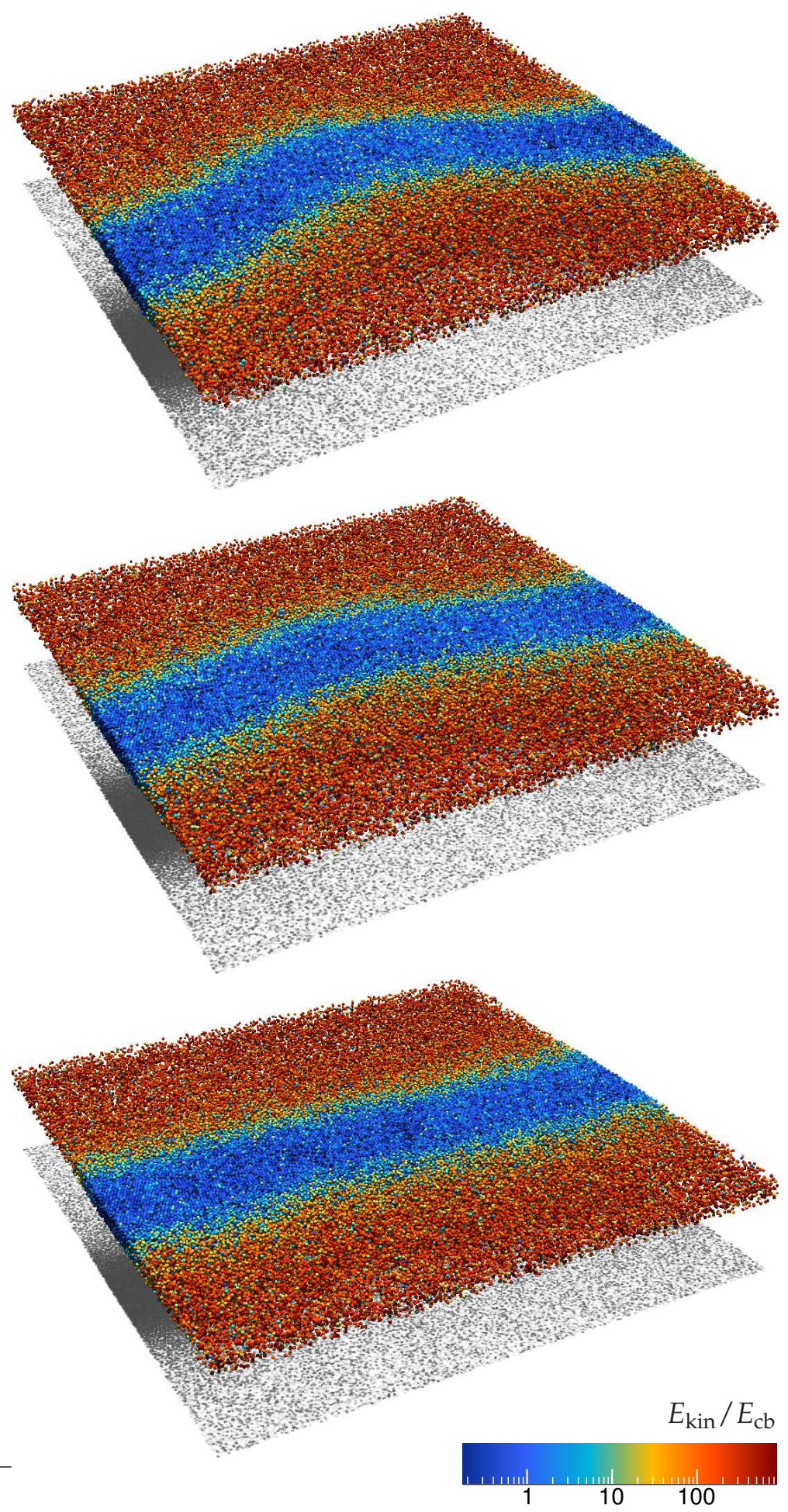

Fig. 5.13 Snapshots of a three-dimensional simulation which shows the relaxation of a deformed state back into a strip state after switching off the external shear force. Time evolves from top to bottom. The system contains $1.2 \times 10^{5}$ elastically colliding particles and its size is $8 d \times 192.5 d \times 192.5 d$. The sinusoidal shaking is done at $\Gamma=100$, and $\tilde{E}^{*}=0.72$. Other simulation parameters are the same as in Fig.5.7. 
exponential decay. At the end of the simulation a stationary state with an almost flat interface is reached. However, the interface undergoes fluctuations which are of the order of a few particle diameters, but can also become larger. A snapshot where such a fluctuation is visible at only one interface of the strip was presented in Fig. $5 \cdot 3$ (middle).

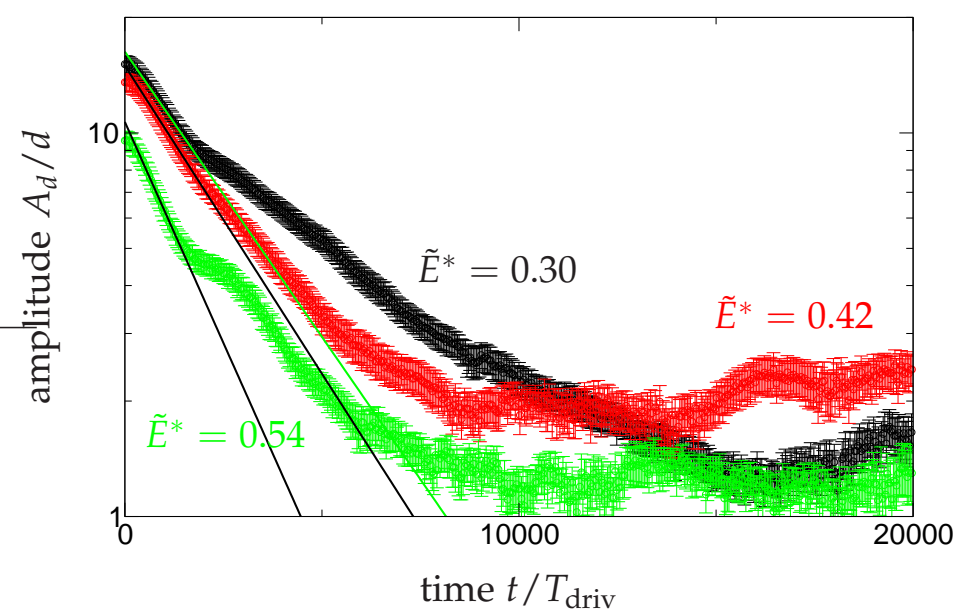

Fig. 5.14 Elongation amplitude, $A_{d}$, normalized by the particle diameter, $d$, is plotted versus time for three different driving energies. The solid lines are exponential fits to the data and lie in a large range of time within the error bars, which denote the standard deviation of the average over 20 individual simulations at slightly different initial conditions. Simulation parameters are the same as in Fig.5.7.

Using the fit to the exponential decay at the beginning of the simulation (lines in Fig. 5.14), the effective mobility, $\mu_{f}$, of the particles which is associated with their self-diffusion was estimated. The resulting effective mobility is shown in Fig. 5.15 and shows strong scattering, but nevertheless a trend for increasing mobility with increasing driving energy can approximately be observed. The strong scattering is not surprising in view of the small range of the data where the decay of the amplitudes followed the exponential decay. The estimation of the mobility shall therefore be considered as a first approximation of this quantity.

In this section, the interfacial energy of a fluid gas coexistence was estimated for the simulations by deforming an initially flat interface. This was done by applying a sinusoidal shear force to the system. Surprisingly, it was found that the deformation amplitude seems to scale linearly with the maximum applied shear force, in accordance with the prediction of a continuum model in equilibrium. This linear scaling is a necessary condition that the method of mechanically deforming the interface can lead to meaningful results in terms of the interfacial energy. The interfacial energy as a function of the driving energy showed good qualitative agreement between the simulation and a simple estimation based on the binding energy of the capillary bridges. The estimate predicted a linear increase of the interfacial energy with the critical rupture separation, which is a suitable topic for further numerical studies. It seems particularly interesting and promising to compare the results obtained by 


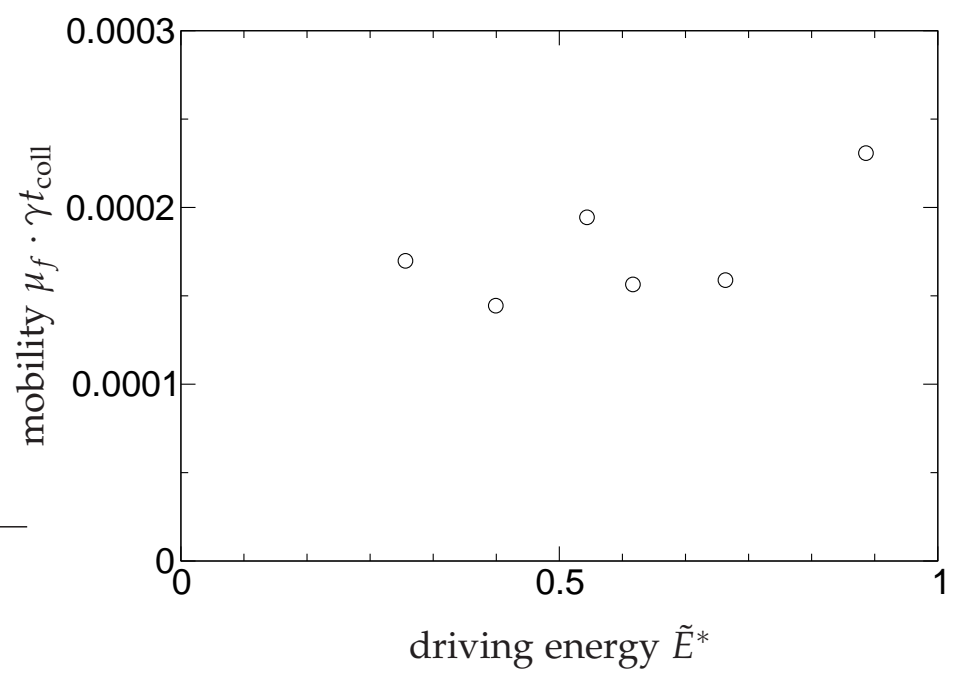

Fig. 5.15 Effective mobility, $\mu_{f}$, as obtained by simulations, plotted against the normalized driving energy, $\tilde{E}^{*}$. The mobility is normalized by the surface tension $\gamma$ of the wetting liquid and the mean time between collisions in the fluid phase, $t_{\text {coll }}$, (estimated as $t_{\text {coll }}=1 / n_{p}^{1 / 3} \sqrt{T_{g}}$ ), where $n_{p}$ is the particle number density and $T_{g}$ the granular temperature.

simulations here with experiments which will be done on this subject in the future.

\subsection{Coarsening dynamics}

In the previous sections it was shown that an interfacial energy seems to be present in three-dimensional, wet granular matter which shows a fluid-gas coexistence. In the present section it will be studied if the existence of an interfacial energy is of appreciable universality with respect to dimensionality and the phases being in coexistence. Finally, the coarsening dynamics is examined in two different wet granular systems which partially show similarities to equilibrium system. Coarsening is first studied in a two-dimensional system showing the fluid-gas coexistence, where the coarsening will be in some parts reminiscent of the coalescence of fluid droplets. In the second part of this section a solid-fluid coexistence is studied in the three-dimensional system, where the coarsening is reminiscent of Ostwald ripening. Due to the complex dynamical behavior in the two-dimensional case no conclusive result can be given. In the case of the solid-fluid coexistence an interfacial energy is suggested by the similarity to the Ostwald ripening.

It was J. W. Gibbs who first realized that the formation of small clusters in the supersaturated ambient phase, induced by small density fluctuations, is a prerequisite for nucleation to occur. Thus, a certain energy barrier has to be overcome (which is increasing with increasing interfacial energy) for the initial nucleus to persist (Gibbs, 1928; Kashchiev, 2000; Markov, 2003). After the formation of multiple nuclei and fast growths of these nuclei into stable domains, the system quests to minimize the total interfacial area between the domains of coexisting phases to reach an ener- 
getically more favorable state. This process is called coarsening because the largest domains grow in size on expense of smaller ones. Several different coarsening mechanisms are known (Voorhees, 1992; Campbell et al., 2002; Bowker, 2002; Becker et al., 2003; Rump, 2008) as for example the two main mechanisms coalescence and Ostwald ripening, where the latter is well described by the Gibbs-Thomson equation (Ostwald, 1900; Lifshitz and Slyozov, 1961; McDonald, 1962; Voorhees, 1992, and references therein).

\subsubsection{Nucleation and coalescence}

Indications were found in the previous section that the interfacial energy possibly is caused by the capillary bridges. No evidence can be found why the simple theoretical estimate of the interfacial energy presented there cannot be applied to a two-dimensional system. Thus, the coarsening dynamics of the two-dimensional, wet granular system is studied. If a behavior reminiscent of an equilibrium system was found, this could give indications that also in the two-dimensional system an interfacial energy is present. At some stages a behavior is found which is reminiscent of a coalescence process. However, on the long run the system shows much more complex dynamical behavior which eludes of any simple description and thus the presence of an interfacial energy could not be clearly shown.

The emerging coarsening dynamics in simulations is presented and described in detail. Then a simple theoretical estimate on this topic is reviewed and its limits of validity are discussed.

\subsubsection{Observation}

To study nucleation and coarsening processes in wet granular matter, event-driven simulations of a two-dimensional system which is hundred times wider than in the previous section are performed. The system contains $1.2 \times 10^{5}$ elastically colliding disks which are interacting via the thin-thread model. At the beginning of the simulation the particles are distributed in the system on a regular lattice and obey a Gaussian velocity distribution with mean granular temperature $T_{g} / E_{\mathrm{cb}}=20.0$. Other system parameters are the same as in Fig. 3.2

A detailed description of what can be observed in the simulations shown in Fig. 5.16 and 5.17 will now be given. Fig. 5.16 shows the areal density (grey scale) as a function of time (horizontal axis) and lateral position (vertical axis) on the top. The areal density is normalized by the maximum packing fraction, $\phi_{\max }$, which is measured during simulation in order to maximize the contrast of the resulting image. At the bottom the diagram shows the mean granular temperature during the simulation (red curve) as well as the fraction of the system, which is occupied by the fluid droplets (black curve). For the simulation in Fig. 5.17 only the microscopic distribution of initial particle velocities was different, all other system parameters were the same. The figure shows the capillary bridge density (black: many bridges, 
white: no bridges) on a linear scale. At the bottom of the figure two magnifications of regions of interest are shown, which reveal the details of the dynamics much better.

Five different regimes are distinguished in Fig.5.16 and termed as follows: 1) The nucleation regime in the intervall $\left.t<t_{0} ; 2\right)$ the coalescence regime in the intervall $t_{0}<t<t_{1}$; 3 ) the reordering regime in the intervall $t_{1}<t<t_{2}$; 4 ) the re-nucleation regime in the intervall $\left.t_{2}<t<t_{3} ; 5\right)$ the final stationary regime in the intervall $t>t_{3}$.

The nucleation regime is dominated by precipitation of fluid nuclei out of the initially homogeneous gas phase. This can be seen best in Fig.5.17(1), where five fluid droplets nucleate out of the initial gas phase. It seems as if there is a preferred separation length of two adjacent fluid regions. To support this observation the resulting probability density of the separation lengths in the system is shown in the inset of Fig.5.16, indeed suggesting a maximum between $100 \mathrm{~d}$ and $200 \mathrm{~d}$.

The nucleation is followed by the coalescence regime. The fluid droplets seem to start attracting each other and successively coalesce with adjacent ones to form larger fluid droplets. It is best seen in the zoom into the region (I) in Fig.5.17. The attraction seems to result from a local decrease in pressure which possibly is caused by the stronger dissipation at the fluid-gas interface (cf. Fig.5.18). This causes driving forces perpendicular to the driving wall 5 . The process of coalescence reduces the interfacial area and seems to suggest the presence of an interfacial energy.

In the reordering regime it becomes visible that the system does not continuously reduce its interfacial area. A phenomenon can be be observed where a gas bubble is periodically arising within a fluid regime and therefore the interfacial area is increasing. This is the situation which is highlighted in Fig.5.17(2). This periodic arise of the bubble can be qualitatively understood. Dissipation mostly occurs in the dense phase and especially at the interface, whilst energy injection occurs in the gas phase. This implies a resulting net heat current from the hot gas to the cold fluid drop, where the energy is dissipated. Since the number of interfaces is decreasing through coalescence, the dissipation becomes less effective. As soon as the heat current exceeds a critical value, i. e. transporting more energy than can be dissipated locally, this leads somewhere inside the fluid drop to the emergence of a small gas bubble. The creation of a new gas bubble increases the number of interfaces and a larger portion of the heat current can be dissipated locally. The gas cools down and condensates again. If the heat current is still not reduced, this process will repeat periodically and eventually result in a complete evaporation of the fluid drops. If this occurs all over the system, this leads to a drastic increase in granular temperature, which can be seen at time $t_{2}$ in Fig.5.16. At the same time the whole system seems

5. Lateral homogeneity of the pressure is regained in the stationary state. A first step towards a rough theoretical description of the coalescence dynamics would be the inclusion of time-dependent lateral pressure inhomogeneities in to the model shown in Sec. 3.2 


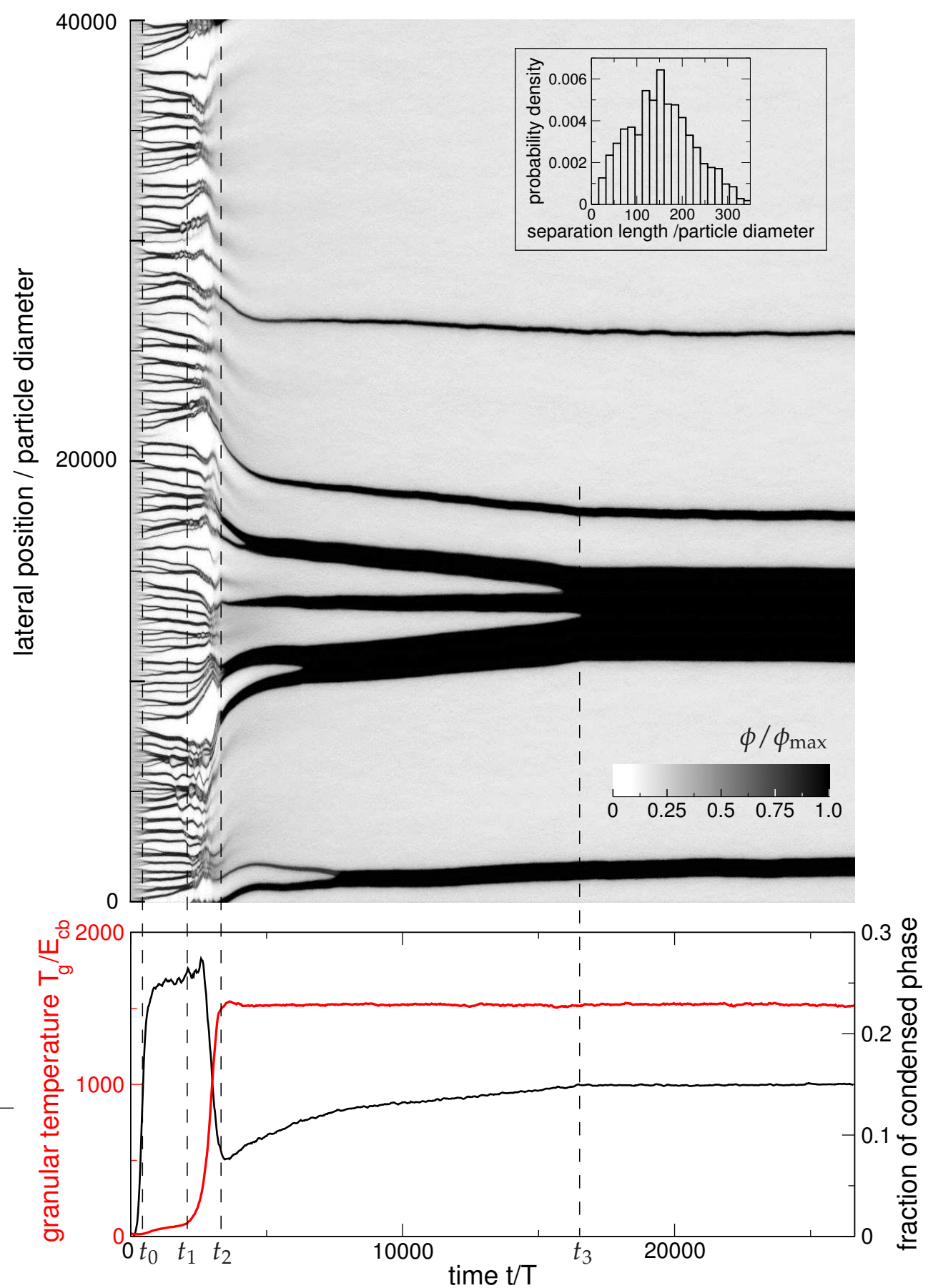

Fig. 5.16 The areal density (top) of a two-dimensional event-driven simulation of $1.2 \times 10^{5}$ elastic harddisks using the thin-thread model is shown as grayscale image as a function of the time and the lateral position (averaged in the vertical direction) and normalized by the maximum packing fraction obtained during the simulation, $\phi_{\max }$. The system is almost the same as used in Fig. 3.2, but hundred times wider as previously (system dimensions: $9 d \times 40000 d$ ) and also using hundred times more particles. The inset shows the probability density to find a certain separation length between two adjacent fluid droplets at a time between $t_{0}$ and $t_{1}$. The mean distance is found to be $157 d$. Below the figure the mean granular temperature, $T_{g}$, is shown and also the fraction of the system, which is occupied by the fluid droplets. 

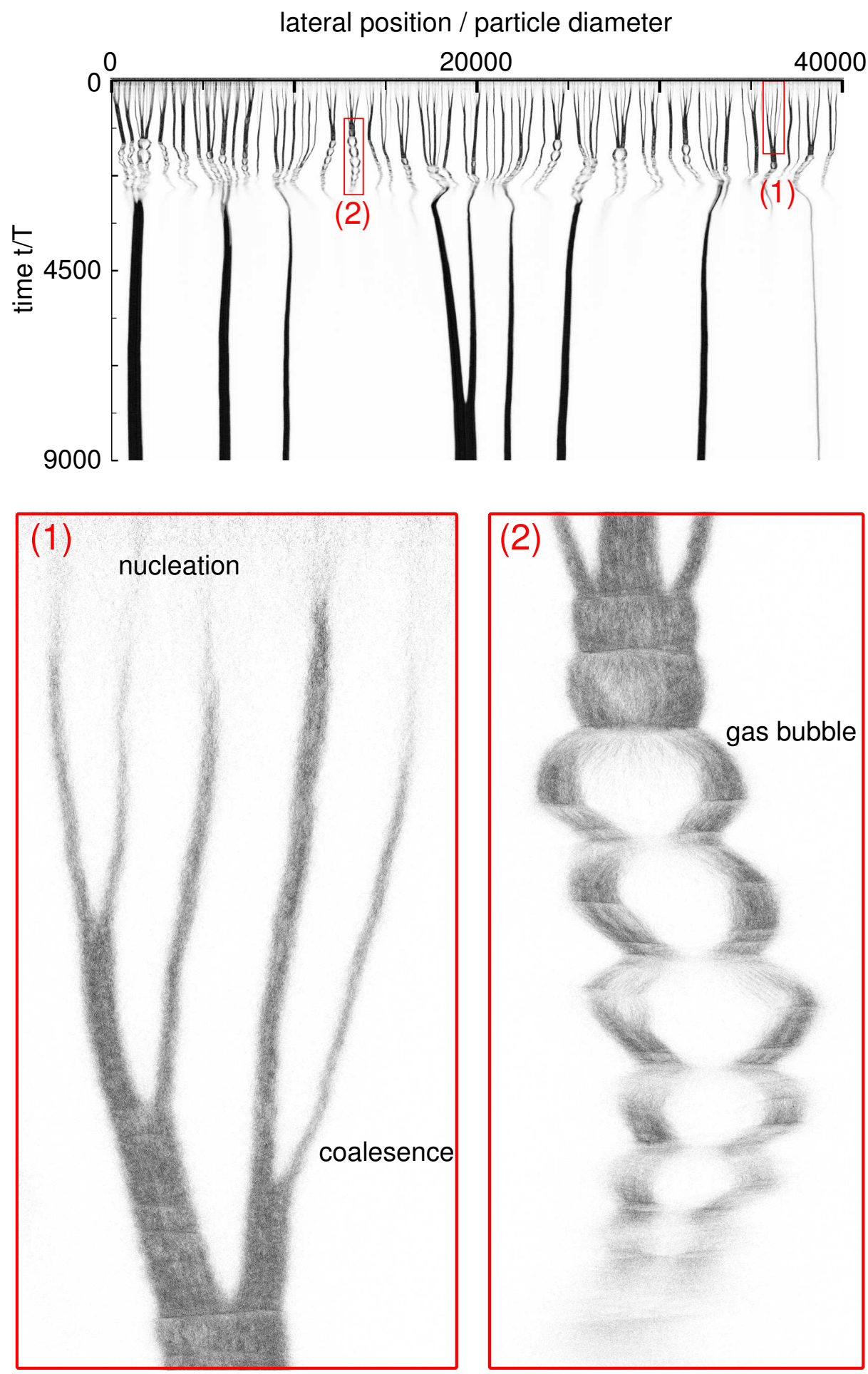

Fig. 5.17 The bridge density of a similar simulation as in Fig.5.16 is shown again as a function of lateral position and time (top). The regions marked by red rectangles and labeled as (1) and (2) are magnified below the image. In magnification (1), an oscillatory occurrence of a gas bubble is visible, where a single dense fluid drop periodically becomes divided by a gas phase growing inside the fluid droplet. Due to the increased number of interfaces, the dissipation becomes more effective and cools down the system again leading the two droplets to almost merge again. In magnification (2), the nucleation process is clearly obtained. After nucleation there seems to be attraction between different fluid droplets, which leads to successive coalescences of fluid droplets. The system and driving parameters are the same as in Fig. 5.16 only using a different random seed for the initial velocity distribution. 
to be in a gas state only showing lateral inhomogeneities in the areal density ${ }^{6}$.

Surprisingly 7 , in the re-nucleation regime a few fluid droplets start to nucleate out of the spatially inhomogeneous gas phase and again start to reduce the global interfacial area through coalescence again.

In the final stationary regime it seems as if all quantities that are shown in Fig. 5.16 are stationary with four fluid regions (of very different size) left. If there is interfacial energy in the system it would be expected that the coalescence proceeds, although very slowly, until only one fluid drop remains.

The dynamics emerging in the present system seem to be very complex and the observations described above do not suggest an interfacial energy. This is because the interfacial area is temorarily increasing in the reordering regime if there is a gas bubble appearing within a fluid droplet. An interfacial energy in a system in thermal equilibrium would lead to a monotonic decrease of the interfacial area with time. This could clearly not be observed here.

A rough theoretical estimate shall be reviewed which was claimed to seemingly capture the dynamics of this complex process, in what follows.

\subsubsection{Discussion of existing models}

A rough theoretical estimate on describing the coarsening process presented above was suggested in (Hager-Fingerle, 2007, section 10.7). However, the numerical data presented there do not seem very convincing and thus the theoretical estimate shall now be compared with the simulations presented in this thesis.

The estimate in (Hager-Fingerle, 2007, section 10.7) assumes a temperature dependent length scale in the system. Furthermore, this length scale is assumed to scale linearly with the heat conductivity of the gas. This finally leads to the relation $N_{f} \propto L_{x} / \sqrt{T_{g}}$ which predicts the number of fluid droplets $N_{f}$ as a function of granular temperature. The data presented in (Hager-Fingerle, 2007, section 10.7) to support this scaling showed a pure gas state, $N_{f}=0$, at time $t_{2}$ before the renucleation sets in. The temperature of this gas state was shown to be approximately $T_{g} / E_{\mathrm{cb}}=1000.0$. How this is consistent with the theoretical prediction which states that $N_{f}=0$ only occurs at $T_{g} \rightarrow 0$, was not clearly addressed. As it was claimed that the "granular matter reaches its final state without violating the length scale"

6. The lateral inhomogeneities in the density and the small time and spatial resolution of the system seems to lead to the artifact, that the fraction of the system which is occupied by the fluid drops is not detected as being completely zero in Fig.5.16, and similarly leads to an overestimating of the number of clusters in Fig. 5.19.

7. This effect was originally discovered in a ten times narrower system which resided in the pure gas state for almost two days of clock time on a single computer. As computation time is a valuable commodity and there were no expectations to find anything else than a pure gas state, the simulation was planned to be canceled and new ones to be started before leaving the office that night. Only by forgetting this, the author of the present work was amazed at finding a fluid drop again in the system looking at it the next morning. It is remarked that the data of these simulations were used in Fig. 10.18 and 10.19 of (Hager-Fingerle, 2007) accidentally without citation, but fully authorized. 


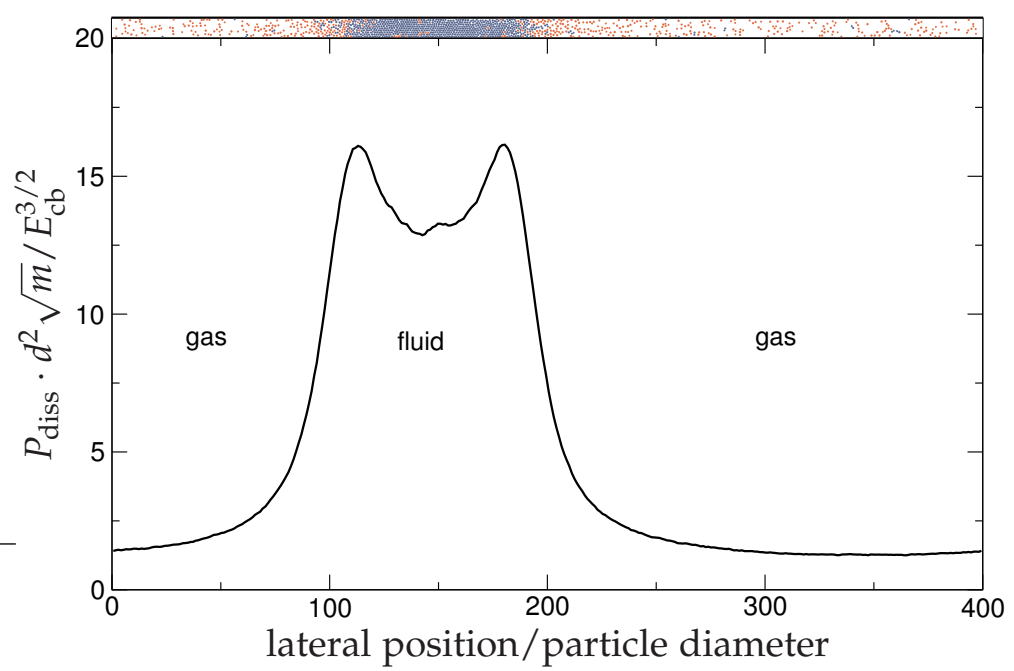

Fig. 5.18 The dissipated power, $P_{\text {diss }}$, is shown in spatial resolution in a fluid-gas coexistence. As it is the same system as in Fig. 3.2 the corresponding temperature and density profiles can be found there. The box on top shows a snapshot of the two-dimensional system. It can be seen that the dissipation reaches its highest value directly at the interface.

(Hager-Fingerle, 2007, pp. 233) this observation seems to falsify the theoretical prediction. The theoretical estimate thus seems not to be sufficient to predict the dynamical behavior at the transition from the reordering to the re-nucleation regime. This is not surprising as the ongoing dynamics at the re-nucleation transition is far more complex than captured by the simple theoretical estimate. However, in the following it will be examined if the theoretical estimate holds within the coalescence regime, where the number of fluid droplets is also decreasing.

The number of fluid droplets as well as the granular temperature is easily accessible in the simulation and thus is shown in Fig.5.19(black circles) for the simulation displayed in Fig.5.16. The red curve is a fit to the data according to $N_{f} \propto 1 / \sqrt{T_{g}}$ and reflects the theoretical prediction. It is in reasonable agreement with the numerical data. Therefore, it seems that the theoretical estimate nicely predicts the increase in granular temperature during the process of coalescence in the wet granular matter. This finding also supports the result shown in (Hager-Fingerle, 2007, Fig. 10.18) for the coalescence regime. There it was shown that the relation $N_{f} \propto 1 / \sqrt{T_{g}}$ (as $N_{f}$ is an integer), predicts a discrete temperature spectrum.

In conclusion, it was shown that the theoretical estimate presented in (HagerFingerle, 2007, section 10.7) seems to predict the increase in granular temperature which occurs along with the decrease in the number of fluid droplets. This leads to a discrete temperature spectrum which implicitly is also confirmed. However, it was also clearly shown that the estimate is not able to predict the transition across different regimes in contrast to what was assumed in (Hager-Fingerle, 2007, section 10.7). 


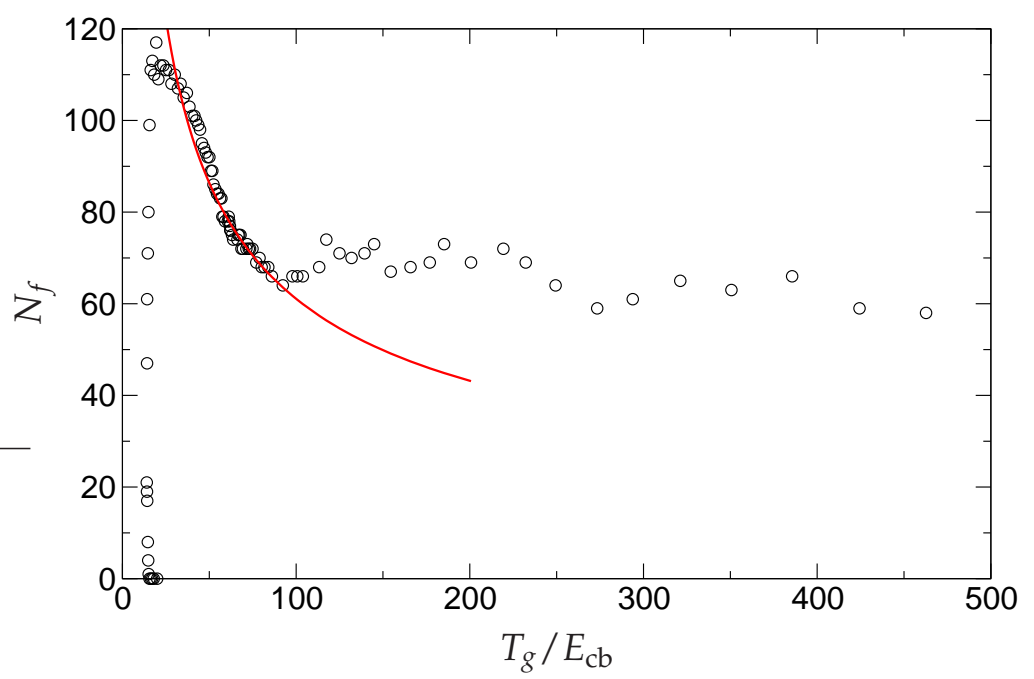

Fig. 5.19 The number of condensed phases, $N_{f}$, in the simulation shown in Fig.5.16 is shown as a function of the mean granular temperature, $T_{g}$. The red line is a fit to the data of the form $N_{f} \propto$ $1 / \sqrt{T_{g} / E_{\mathrm{cb}}}$, which reflects the theoretical estimate presented in (Hager-Fingerle, 2007, section 10.7) and shows reasonable agreement in the initial coalescence regime.

\subsubsection{Ostwald ripening}

In the following, it will be shown that an interfacial energy seems to exist in a solidfluid coexistence of wet granular matter. To this end three-dimensional simulations are performed which reveal a behavior reminiscent of Ostwald ripening which is known from equilibrium systems. The similarity of the Ostwald ripening process is supported by comparing the decrease in the number of crystallized domains obtained by simulations to the predictions made by Lifshitz and Slyozov (1961). Their agreement suggests an interfacial energy in wet granular matter because it is known that Ostwald ripening is driven by interfacial energy in thermal equilibrium ${ }^{8}$.

Event-driven simulations are performed in a three-dimensional, square-based system of size $L=192.5 d$. Its height is $9 d$ and thus can be viewed as a quasi twodimensional system. The capillary interaction is modeled based on the thin-thread model and collisions between particles occur inelastically with a coefficient of restitution of $\varepsilon=0.96$. Collisions between particles and the wall occur elastically. The particles are initially ordered on a regular lattice with a Gaussian velocity distribution of mean granular temperature $T_{g} / E_{\mathrm{cb}}=40.0$. A snapshot of the system showing a solid-fluid coexistence was presented in Fig.3.18. There it can be observed (especially in (b)) that multiple solid domains form within the system. The dynamical behavior of these solid domains shall be studied in the following. To this end several

8. The interfacial energy of solids is a bit more subtle because solids can withstand shear forces. Measuring the interfacial energy is then more difficult as simple methods as the one used above (by deforming the interface) do not measure the interfacial energy alone (Shuttleworth, 1950; Sell and Neumann, 1966). 
snapshots of the capillary bridge density which are averaged over the vertical direction are shown in Fig.5.20. They were taken equidistantly in time. The system cools down and resembles a homogeneous fluidized state at time $\tilde{t}=504$ (first snapshot). A few solid regions start to crystallize out of this fluid state reaching its maximum number in the second snapshot at time $\tilde{t}=5128$. Starting from here a coarsening process sets in such that the number of crystallized domains is decreasing. This can be seen, for example, for the domain marked by an arrow at time $\tilde{t}=9753$, which decreases in size in the following snapshots and vanishes completely in snapshot six at time $\tilde{t}=23626$. This domain did not come into contact with any other solid domain, but remains spatially stationary throughout. Therefore, the granular particles have to be melted from the crystallized domain, diffuse through the ambient fluid phase and precipitate again at a different crystallized domain. This bears striking similarity with the coarsening process which is known as Ostwald ripening. It describes the reduction of interfacial area via a diffusive mass transport from areas of high interfacial curvature to regions of lower interfacial curvature (Ostwald, 1900; Lifshitz and Slyozov, 1961; McDonald, 1962; Voorhees, 1992).

In the following the apparent similarity between the coarsening phenomenon observed in simulations here and the Ostwald ripening will be examined. The first quantitative theory which successfully predicted Ostwald ripening was in the seminal work by Lifshitz and Slyozov (1961). One important assumption involved in their derivation is the conservation of mass in each of the two phases. Their work is known best for their prediction of the universal, self-similar particle size distribution in the long time limit. Yet, several other predictions were made. This is, for example, the prediction for the growth of the domain size, $\bar{R}$, which was obtained to scale as $\bar{R}(t) \propto t^{1 / 3}$. This relation can be used to obtain the scaling for the number of domains, $N_{d}$. It was given in the original work for a three-dimensional system as $N_{d}(t) \propto 1 / t$ but is easily extended to a two-dimensional system where $N_{d}(t) \propto 1 / t^{2 / 3}$ is obtained. This prediction shall be compared to the quasi twodimensional simulation of wet granular matter presented above.

First, the conservation of mass which is assumed in the original theory is examined. Assuming a constant mass density in the crystallized domain, this can be tested in the simulation by measuring the total base area of the crystallized domain. This is shown as the red curve in Fig.5.21. At the initial stage it shows the increase due to the crystallization, but in the coarsening regime at times $\tilde{t}>10000$ it is in good approximation constant.

The number of crystallized domains, $N_{d}$, obtained by simulations is shown in Fig. 5.21 (black circles). The solid black line in Fig. $5 \cdot 21$ is a fit to the data for times $\tilde{t}>9000$ of the form $N_{d} \propto 1 / t^{2 / 3}$. It can be seen that the data seems to resemble the corresponding relation. In order to improve the result larger simulations are needed for longer time-scales. This, however involves considerably more computational power and thus is left for future work. The agreement between the theoretical estimate and the simulation thus suggests that the coarsening phenomenon under study here indeed is reminiscent of Ostwald ripening. Thus it can be concluded that 

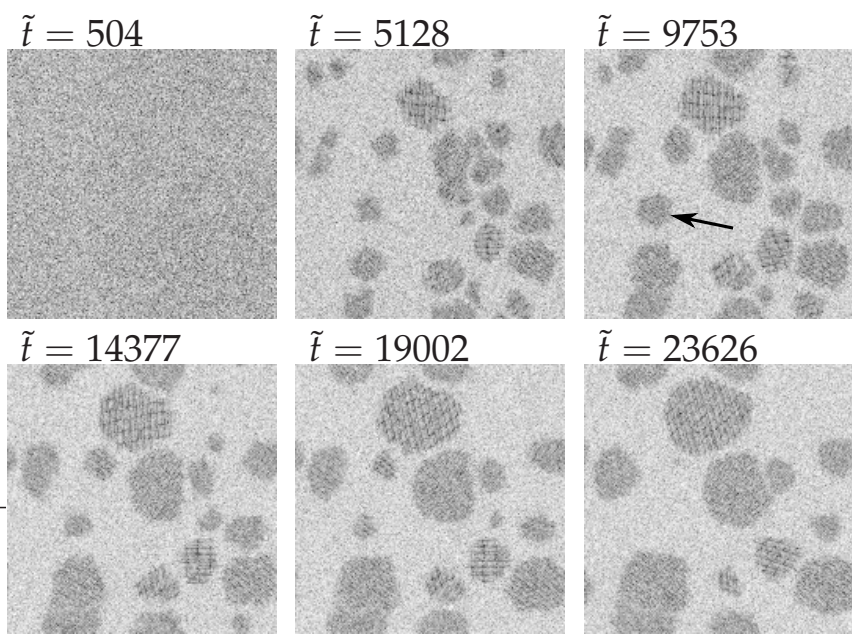

$\tilde{t}=23626$

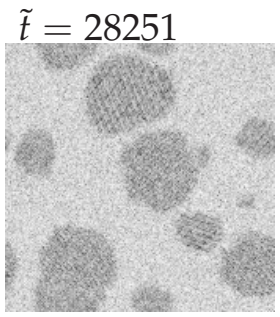

$\tilde{t}=32875$
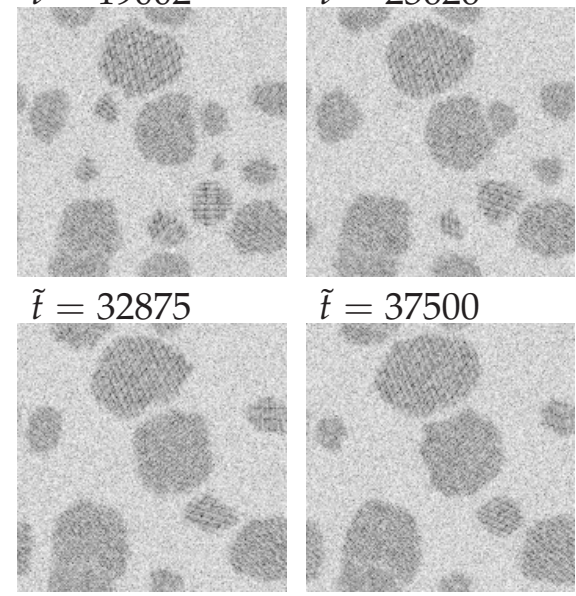

Fig. 5.20 Snapshots equidistant in time, $\tilde{t}=t / T_{\text {driv }}$, of the capillary bridge density are shown. The first snapshot (top left) shows a fluidized state, where in the following snapshot solid phases start to crystallize out of this fluid phase. The number of crystallized regions is decreasing with time, where small drops are shrinking while larger ones grow. All drops remain spatially approximately constant and are only shrinking or expanding. This is indicated, for example, at a small region marked by the arrow which is completely melted three snapshots later. This suggests a diffusive mass transport from areas of high interfacial curvature to regions of lower interfacial curvature reminiscent of the classical Ostwald ripening process. The parameters of the simulation are the same as in Fig. 3.18 with $\Gamma=90.0$ and $E^{*}=1.3$ as driving parameters. The time lag between the snapshots is $\Delta \tilde{t} \approx 4624$. 
the results presented here suggest the a quantity reminiscent of an interfacial energy also in the solid-fluid coexistence of wet granular matter.

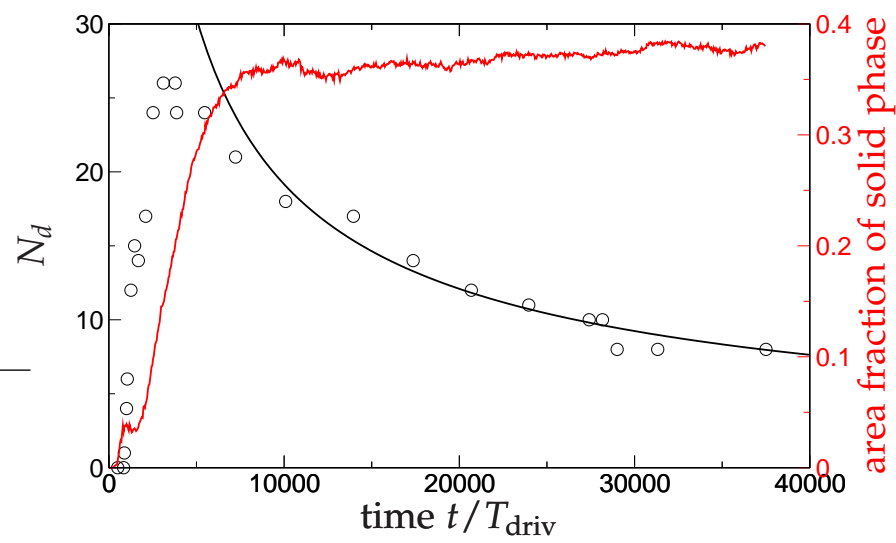

Fig. 5.21 The red curve denotes the total area of the crystallized domains as obtained from the simulation in Fig.5.20. It is normalized by the total area of the system. At the initial stage a crystallization process sets in and the solid domains are growing. At times $t / T_{\text {driv }}>10000$ it approximately remains constant in accordance to the assumptions made in Lifshitz and Slyozov (1961). The number of crystallized domains, $N_{\mathcal{c}}$, (black circles) is similarly changing with time. The initial increase associated with the crystallization process and the decrease at $t / T_{\text {driv }}>10000$ again shows the Ostwald ripening. The solid black line is a fit to the data of the form $N_{c} \propto\left(t / T_{\text {driv }}\right)^{-2 / 3}$ which is the prediction for Ostwald ripening in a two-dimensional system. A snapshot of the system under study can be seen in Fig. 3.18 and is quasi two-dimensional. Thus the data points approximately agree with the prediction in the Ostwald ripening regime $t / T_{\text {driv }}>10000$.

\subsection{Anomalous diffusion in fluid-gas coexistence}

In this section the anomalous diffusion of a fluid plug in wet granular matter will be studied in a two-dimensional system. A conjecture is made that the super-diffusive behavior which is found could possibly be caused by an interfacial energy.

The British botanist Robert Brown was not the first to observe Brownian motion. However, after he had published his observations (Brown, 1828, 1829), the effect of erratically moving pollen on water, which is caused by a "molecular bombardment" (Nelson, 1967), was named after him. It was Einstein (1905) and von Smoluchowski (1906) who were finally able to derive the relation between the diffusion coefficient and other thermodynamic quantities, such as temperature, which is nowadays known as Einstein-Smoluchowski relation. After introducing stochastic calculus, Brownian motion was modeled as a random walk described by a Wiener process, which is now the standard stochastic process (Nelson, 1967; Karatzas and Shreve, 1991). The Wiener process is also widely used in financial mathematics, such as the Black-Scholes model (Black and Scholes, 1973), which was awarded a Nobel Prize in 1997. 
Real systems, however, often show anomalous diffusive behavior (Bouchaud and Georges, 1990; Morita, 1996), which means that there is sub- as well as super-diffusive behavior, where the mean squared displacement does not increase linearly in time, but with an exponent smaller or larger than one. Anomalous diffusion can be observed in a large variety of systems ranging for example from networks (Brockmann, 2003, and references therein), biological systems (see e. g. Caspi et al., 2002), geological systems (see e. g. Richardson, 1926; Cushman et al., 2009, and references therein) or granular matter (D'Anna, 2003; Khan and Morris, 2005; Mayor et al., 2005; Dufty and Brey, 2005).

\subsubsection{Observation}

Usually, when studying Brownian motion, a large intruder particle is immersed in the system and the forces causing its motion are monitored. Here, instead of using an intruder particle, the dense fluid phase itself serves as a 'plug' with larger mass. As the system height is only 9 particle diameters, the plug is in a quasi onedimensional system which is anisotropic (cf. Fig.3.2). Because the motion of this condensed region becomes mostly visible on long time-scales, the simulations are performed for a two-dimensional system of 1200 particles, but on up to 100-times longer time-scales than typically used when studying stationary states. The capillary bridge density, where black indicates many bridges (fluid) and white indicates no bridges (gas) in Fig. 5.22 , is used to visualize the motion of the condensed phase with time within the system. The simulations in Fig.5.22(a)-(c) have been performed with the same parameters and only varying the random seed to obtain different initial velocity distributions. It can be seen in (a)-(c) that there is no preferential direction of the motion and the condensed phase is erratically moving. In Fig. 5.22(d) the small range of subfigure (c) indicated by the vertical lines is shown. On short time-scales small fluctuations can be observed but no macroscopic motion is visible. Therefore, taking time-averages in the stationary state is possible, if the time-scales on which the averaging takes place is small compared to the time-scales on which the random motion appears.

The red and green line in Fig. $5 \cdot 22(\mathrm{~d})$ is the interface between the fluid and gas phase. The arithmetic mean of these two lines accounts approximately for the motion of the center of mass and is plotted in Fig. 5.23 for six different initial conditions. As there is no friction between the particles and the top and bottom wall confining the system, there is no damping mechanism which counteracts the lateral motion. Clearly an erratic motion is visible, which is reminiscent of Brownian motion. The width of the plug also undergoes some fluctuations with a standard deviation of less than three particle diameters. This is less than one percent of the system width and close to the resolution limit which is approximately one particle diameter and therefore neglected here. 
(a)

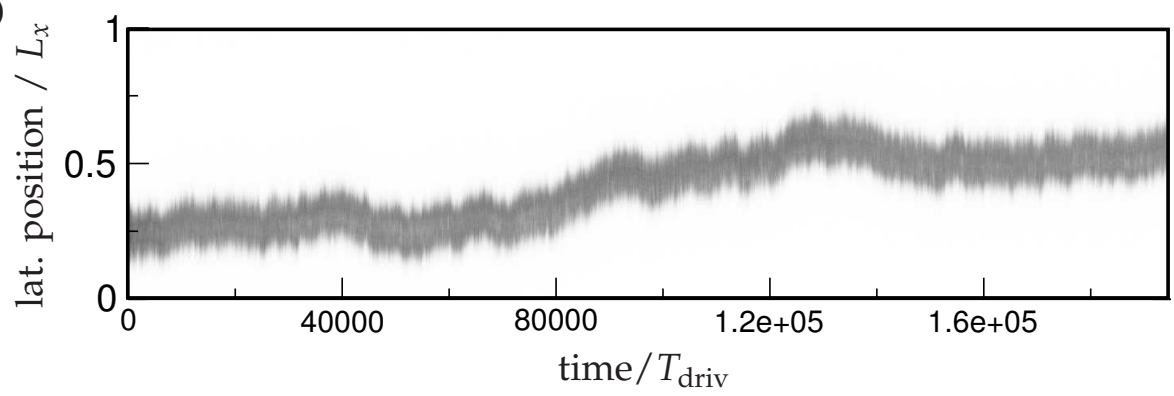

(b)

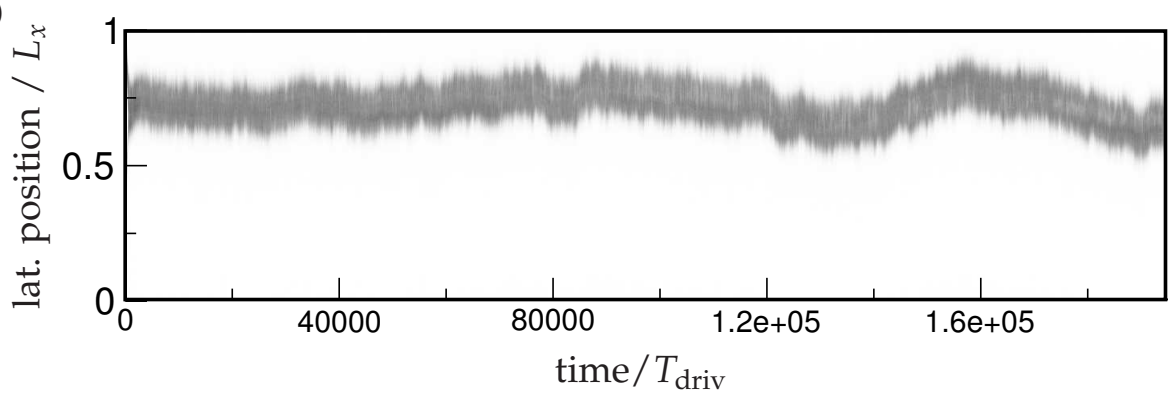

(c)

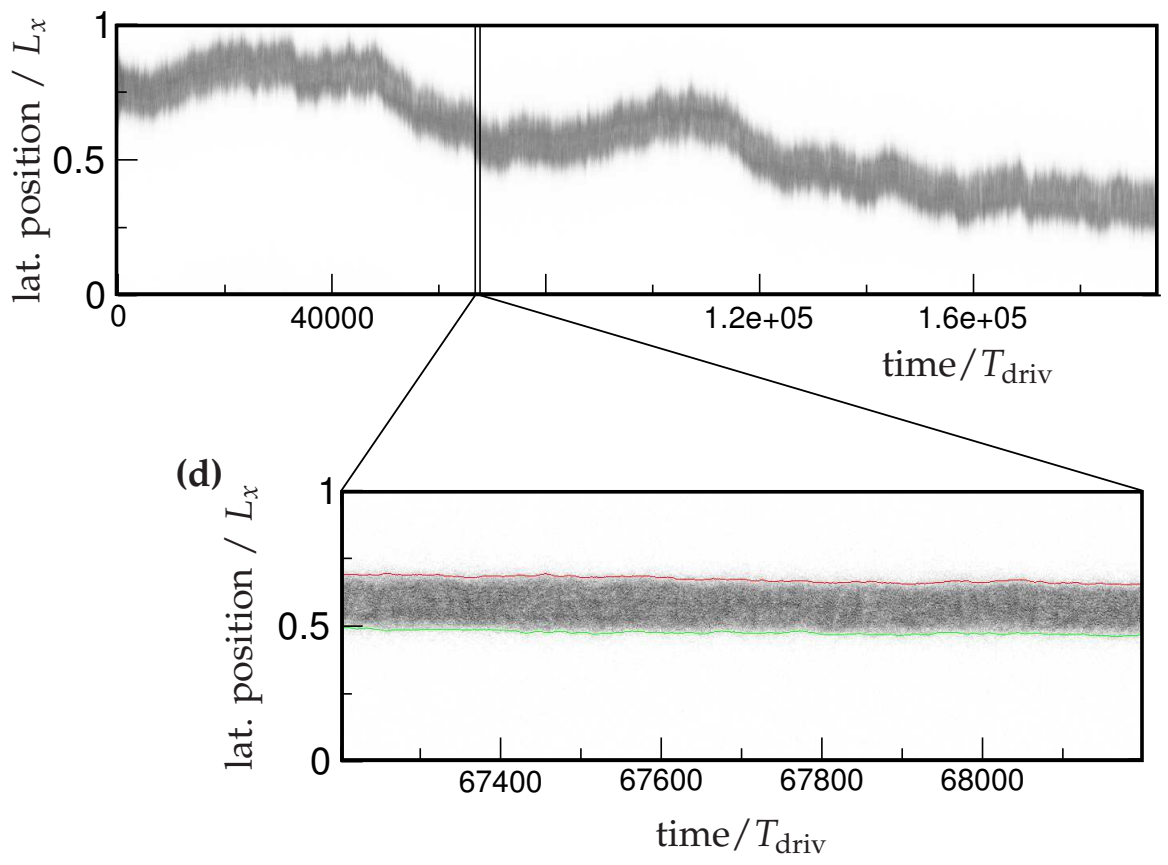

Fig. 5.22 The capillary bridge density (gray scale) is shown for a two-dimensional simulation of 1200 particles in spatial resolution versus time. The time is normalized by the period of the sinusoidal driving $T_{\text {driv }}$. The system and the driving parameters are the same as the one used in Fig.3.2. Three different random seeds were used in (a)-(c) to obtain slightly different velocity distributions as initial conditions. The dense fluid phase is visible as dark gray area and no preferential direction of motion is visible. Subfigure (d) shows a small range of the simulation shown in (c) as denoted by the vertical lines and demonstrates that the macroscopic motion of the condensed phase is only visible on large timescales. The red and green line denote the boundaries between the fluid and gas phase as automatically detected. The width of the system is $L_{x}=400 d$, while the height of the system is $9 d$, where $d$ is the particle diameter. The driving parameters for the sinusoidal shaking are throughout this section $\Gamma=15.0$ and $E^{*}=1.42$. The rupture distance, normalized by the particle diameter, is $s_{\text {crit }} / d=0.0711$, the capillary bridge energy is $E_{\mathrm{cb}} / m g s_{\text {crit }}=4.0$, the mass of one particle is $m=1$, while acceleration due to gravity is $g=1$. 


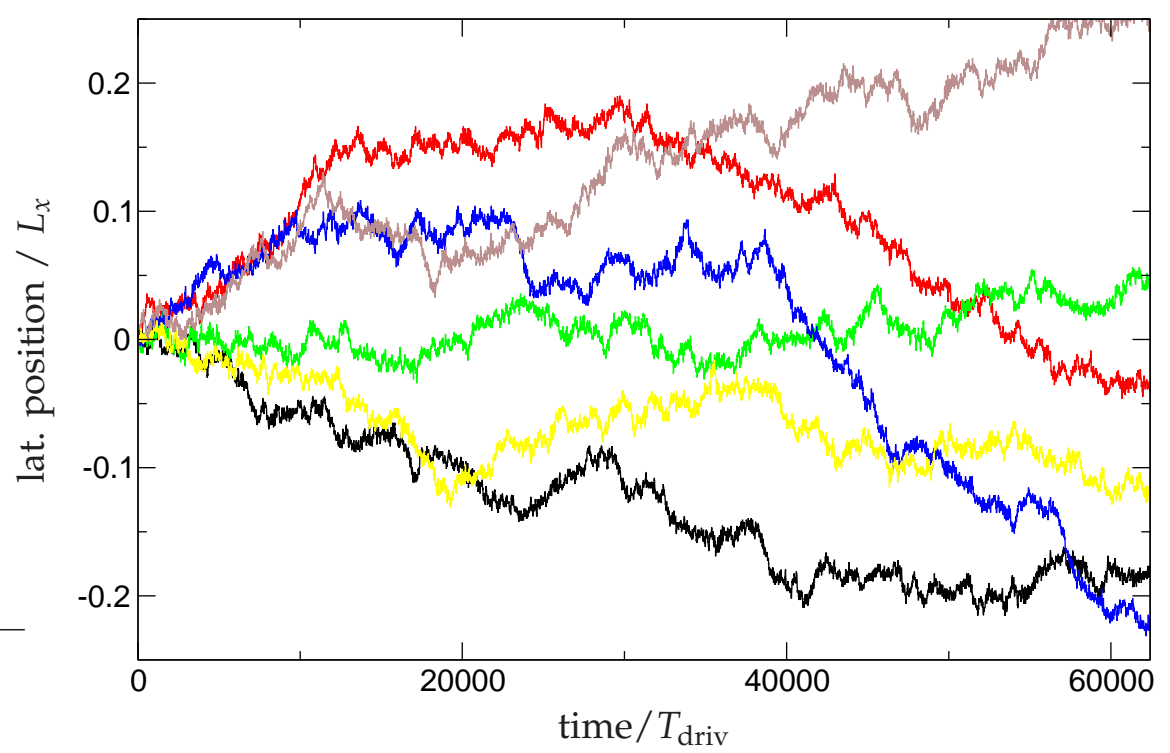

Fig. 5.23 Lateral position of the center of mass of the condensed phase plotted versus normalized time as above. Different colors indicate different random seeds for the initialization of the velocity distribution. All parameters of the simulation are the same as given in Fig.5.22.

\subsubsection{Results}

It is necessary to calculate the mean squared displacement in order to justify the similarity with Brownian motion. Therefore, the mean squared displacement $\sigma_{\mathrm{rw}}$ of the center of mass position of the plug is calculated for 450 different simulations and shown as a function of time in Fig.5.24 (black curve). The simulations differed in the sense, that every simulation used a different random seed to initialize the gaussian velocity distribution with mean granular temperature $T_{g} / E_{\mathrm{cb}}=20.0$. As already pointed out, it can be see that the mean squared displacement vanishes on short timescales. For larger times $(\tilde{t}>1000)$ the red curve is a fit to the data of the form $\sigma_{\mathrm{rw}} / L_{x}^{2}=D_{x}\left(t / T_{\text {driv }}\right)^{\alpha_{\mathrm{rw}}}$, where the diffusion constant is obtained as $D_{x} \approx 1.6 \times 10^{-8}$ and the diffusivity exponent is obtained as $\alpha_{\mathrm{rw}}=1.27$ indicating super-diffusive behavior. However the range where the data follows the fit is hardly one decade. Thus the inset of Fig. 5.24 extends the simulated times by a factor of three but is only calculated for 80 different simulations. The diffusion coefficient here is obtained as $D_{x}=4.1 \times 10^{-9}$ and the diffusivity exponent is $\alpha_{\mathrm{rw}}=1.38$, reasonable close to the values obtained for the shorter simulation time.

Brownian motion is qualitatively predicted by a one-dimensional model which conserves momentum and energy (von Smoluchowski, 1906). The motion of the intruder particle then comes from collisions with neighboring particles which collide with the intruder at statistically distributed times and with statistically distributed velocities. These fluctuations in the collisions lead to a random motion with growth of the mean squared displacement of the intruder. In the coexisting state of wet 


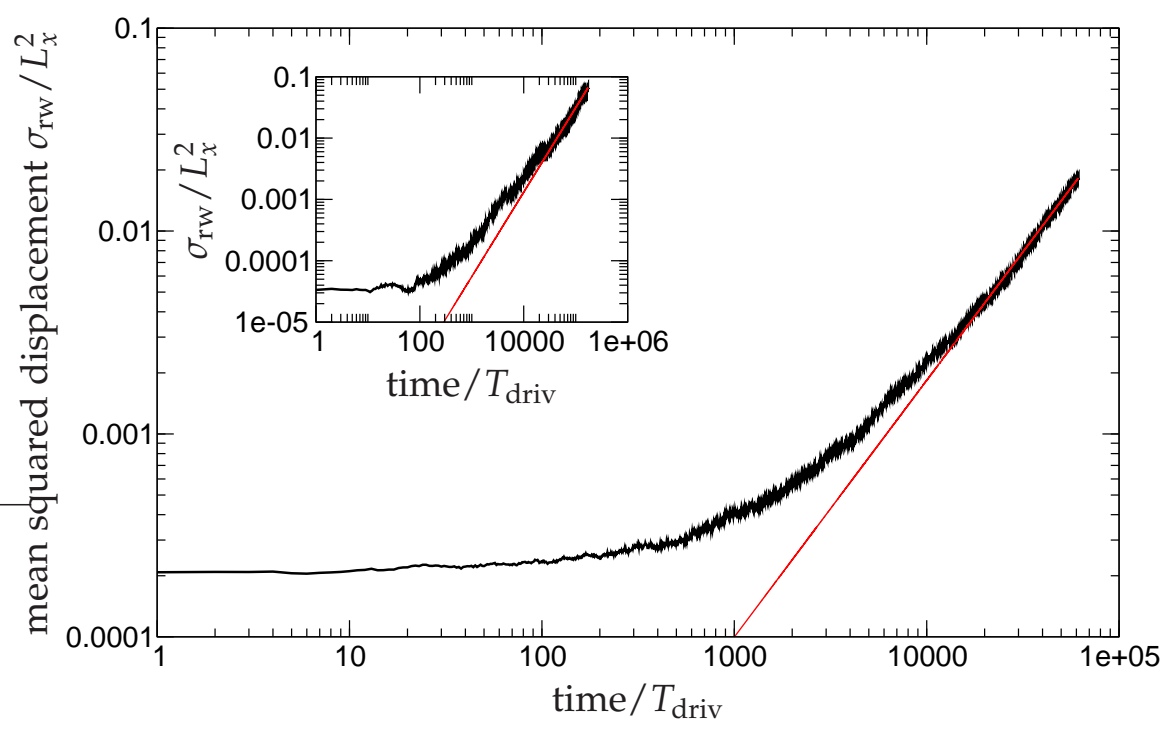

Fig. 5.24 The time dependence of the mean squared displacement is plotted for 450 simulations using different initial velocity distributions (black curve). The red curve is a fit to the data of the form $\sigma_{\mathrm{rw}} / L_{x}^{2}=D_{x}\left(t / T_{\text {driv }}\right)^{\alpha_{\mathrm{rw}}}$, where the diffusion constant is obtained as $D_{x} \approx 1.6 \times 10^{-8}$ and the diffusivity exponent is obtained as $\alpha_{\mathrm{rw}}=1.27$ showing super-diffusive behavior. The inset of Fig. 5.24 extends the simulated times by a factor of three but is only calculated for 80 different simulations. The diffusion coefficient here is obtained as $D_{x}=4.1 \times 10^{-9}$ and the diffusivity exponent increases is $\alpha_{\mathrm{rw}}=1.38$ All parameters of the simulation are the same as given in Fig. 5.22 .

granular matter studied here, this mechanism of momentum transfer is also similarly present, as the particles collide elastically, although the dynamics is dissipative in the long run. However, there is an additional mechanism induced by the capillary interaction which influences the motion of the condensed phase in wet granular matter. Imagine a one-dimensional system, where a particle is colliding with the plug and a capillary bridge is formed. If the kinetic energy of the particle is less than the capillary bridge energy the capillary bridge cannot rupture afterwards. Thus, the plug grows at its surface in the direction where the particle came from, and thus shows surface growth. As the condensed plug has two interfaces with the surrounding gas phase and the size of the plug is stationary this will lead to a stochastic motion of the plug. Thus the surface growth process is a second mechanism which induces stochastic motion. Summing up these two mechanisms (momentum transfer via collisions and surface growth) there has to be a non-trivial coupling the two between in order to explain the deviation from the classical equilibrium diffusivity exponent, $\alpha_{\mathrm{rw}}=1$. The origin of this non-trivial coupling is not easily seen. However, the non-linearity in the KPZ equation, which describes surface growth processes also in non-equilibrium systems, indicates that this could possibly be a reason. This is interesting in the perspective of recent findings, where "the weakly asymmetric simple exclusion process approximation was used to establish that the variance of the two-point function of the stationary $\mathrm{KPZ}$ equation asymptotically 
behaves as $t^{4 / 3}$ (Balazs et al., 2009)" (Sasamoto and Spohn, 2010). This is close to the exponent of $\alpha_{\mathrm{rw}}=1.38$, which was found for wet granular matter on the longer time-scales. If it turns out that this similarity is not just a pure coincidence, this would be a particularly interesting result, because it would connect wet granular matter as a realistic system to the KPZ equation for which analytical results can be obtained.

A possibility is suggested how to support or easily falsify this conjecture. The diffusivity exponent obtained for the KPZ equation is independent of the interfacial energy. Thus, by changing the interfacial energy the conjecture can be falsified if the exponent changes. If it stays constant it supports the conjecture. In the previous section it could not be shown that something reminiscent of an interfacial energy does exist in the two-dimensional system of wet granular matter. Thus this is still an open question. In three dimensions something reminiscent of an interfacial energy was clearly obtained and its change along with the driving energy was presented in this chapter. However it was not shown that the anomalous diffusion can similarly be obtained in such a three-dimensional system. At present, the second path seems to be the more promising one and serves well for a future study.

In classical theory of Brownian motion the diffusion constant is independent of the mass of the intruder particle (von Smoluchowski, 1906). As shown before, the size of the fluid phase is subject to change if the driving energy, $E^{*}$, is changed. Thus, the mass of the plug is changing. Therefore, as the granular temperature slightly differs for different driving energies, $\tilde{E}^{*}$, the random motion could probably be studied as a function of the granular temperature. This also serves well for further investigation.

It was shown in this section that the condensed phase in a two-dimensional system of wet granular matter in a fluid-gas coexistence state undergoes macroscopic motion which is erratic and therefore reminiscent of classical Brownian motion. The diffusivity exponent, however, was found to be approximately $\alpha_{\mathrm{rw}}=1.38$ on the longest time-scales simulated and thus shows super-diffusive behavior.

\subsection{Summary and Outlook}

The size of the gas bubble emerging in a fluid-gas coexistence of three-dimensional granular matter was studied and found to depend mainly on the driving energy. This was in good qualitative agreement with experiments. In simulations, different shapes of the fluid-gas coexistence were found: a circular shape of the minority phase immersed in the majority phase and a strip shape. The strip shape is caused by the periodic boundaries in two lateral directions. This situation is reminiscent of a coexistence state found in the two-dimensional Ising model in thermal equilibrium, where the transition between the two shapes gives rise to a first order phase transition and is driven by minimization of the interfacial energy. In the wet granular system circular shapes are observed which seem to show an excess interfacial area compared to a corresponding strip state. Most striking however, is that this also 
suggests a quantity reminiscent of an interfacial energy in the fluid-gas coexistence of wet granular matter.

The interfacial energy of a fluid-gas coexistence was estimated in simulations by deforming an initially flat interface. This was done by applying a sinusoidal shear force to the system. It was found that the deformation amplitude seems to scale linearly with the maximum applied shear force, in accordance to the prediction of a continuum model in equilibrium. This linear scaling is a necessary condition that the method of mechanically deforming the interface can lead to meaningful results in terms of the interfacial energy. The interfacial energy as a function of the driving energy showed qualitative agreement between the simulation and a simple estimation based on the binding energy of the capillary bridges. The estimate predicted a linear increase of the interfacial energy with the critical rupture separation, which is suitable for further numerical study.

An investigation was conducted to determine if an effect reminiscent of interfacial energy can be found in a two-dimensional fluid-gas coexistence and a threedimensional solid-fluid coexistence of wet granular matter. The first case revealed complex dynamical behavior which seems to be reminiscent of coalescence of fluid droplets in certain regimes. The presence of an interfacial energy was not suggested by the simulations. This is due to the increase in interfacial area in the reordering regime by the formation of gas bubbles. This increase of the interfacial area is in clear contradiction to what is expected for an interfacial energy driven effect in thermal equilibrium. Nevertheless, the existence of an interfacial energy was not disproven. No obvious reason can be thought of why the simple estimate of the interfacial energy, which worked reasonably well in the three-dimensional case, should no longer hold in the two-dimensional case. The second case of the solid-fluid coexistence in a three-dimensional system demonstrated behavior which was reminiscent of Ostwald ripening. This was supported by the scaling relation obtained for the reduction of crystallized domains with time. As Ostwald ripening in thermal equilibrium systems is known to be driven by minimization of the interfacial area, this suggests that a quantity like an interfacial energy is also present in the solid-fluid coexistence of wet granular matter.

This chapter ended with a study of anomalous diffusion in a two-dimensional fluid-gas coexistence regime. A super-diffusive behavior was suggested by the numerical data. 


\section{The granular Leidenfrost effect}

A drop of water hovers above a surface if the surface is sufficiently hot. It forms a Leidenfrost drop. This effect is also visible in simulations of wet grains if enough layers of particles are stacked upon each other. A condensed plug of grains floating on top of a dilute gas cushion is observed and, due to the compressibility of the gas, the plug undergoes an oscillation. The oscillation is undamped, and its frequency can be understood by modeling the gas as a Van der Waals gas, which takes excluded volume effects into account. Additionally, a Rayleigh-Taylor instability is found if the lateral system size exceeds a certain value. This effect is also contained in the theoretical prediction.

\subsection{Introduction}

\subsubsection{Classical Leidenfrost effect with water}

The Leidenfrost effect is named after the German physician Johann Gottlob Leidenfrost, who studied the behavior of water drops on a very hot steel plate in detail and published his results in a treatise in 1756 (Leidenfrost, 1756). The first observation, however, dates back to 1732 by Herman Boerhaave. He observed that a water drop placed on a steel plate at a temperature above $220^{\circ} \mathrm{C}$ (for water) floats around and lives much longer than one would expect. Such a drop does not boil but shrinks soundlessly with time until it finally disappears. It can last for minutes for a millimeter-sized drop until it is completely evaporated. The experimentally measured lifetime in a recent experiment (Walker, 1988) is shown in Fig.6.1a. The water at the bottom side of the drop immediately evaporates and forms a vapor cushion on which the drop floats (cf. Fig.6.1b). The comparatively very small heat conductivity of the gas strongly suppresses heat conduction between the drop and the solid plate. The drop slowly evaporates mostly due to heat radiation of the hot surface. Leidenfrost measured a typical vapor layer thickness of only a tenth of a millimeter between the solid plate and the drop by illuminating it with a candle from behind. The Leidenfrost effect has been studied in much more detail in recent work (Walker, 

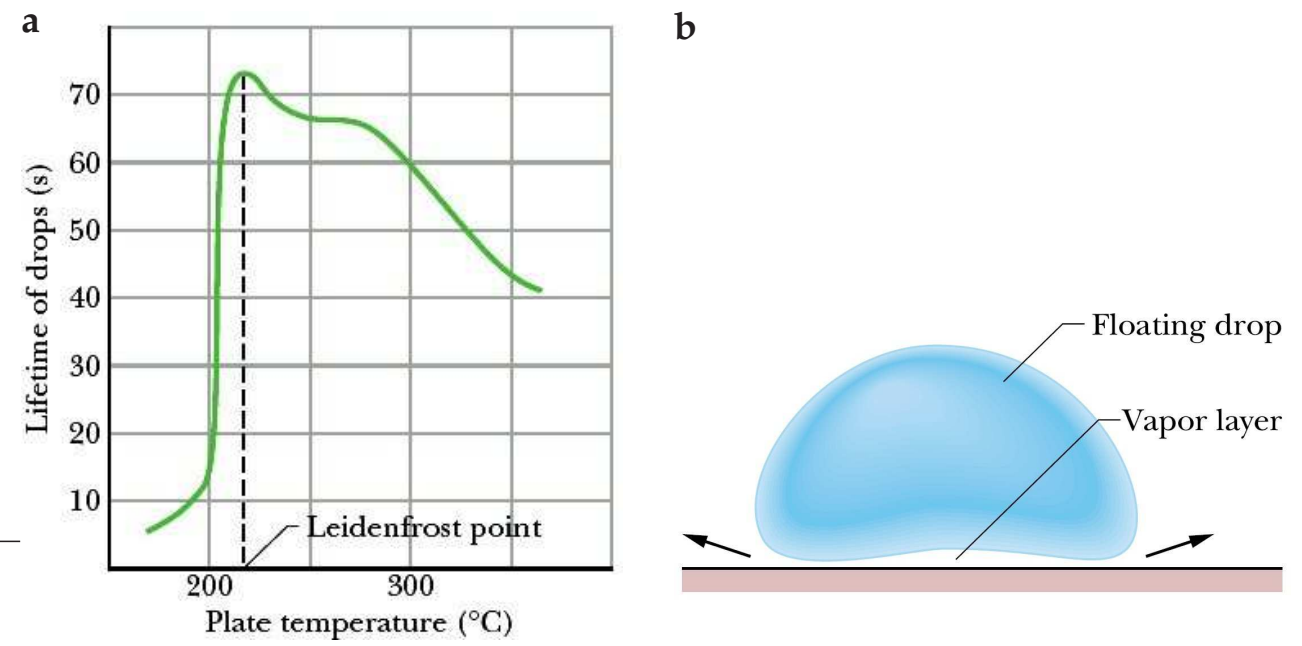

Fig. 6.1 a) Lifetime of a water drop placed on a hot surface as a function of the temperature of this surface. At a critical temperature, the Leidenfrost point, the lifetime strongly increases. b) Cross section of a water drop above the Leidenfrost temperature. The drop is hovering above the hot surface on a vapor layer. Pictures taken from (Walker, 1988).

1988; Biance et al., 2003) and led to new applications in various fields ${ }^{1}$.

\subsubsection{Leidenfrost effect in dry granular matter}

We now look at more complex fluids like granular matter. In contrast to classical liquids, granular particles are of macroscopic order. In order not to loose any of them, the sample is therefore put into a closed container. In simulations the container can,

1. In the field of self-propelled droplets the almost frictionless movement of Leidenfrost drops was the inspiration for a new approach. A device consisting of a metal plate with a periodic, asymmetric sawtooth profiled surface with a height of the steps being $300 \mu \mathrm{m}$ and the length about $1 \mathrm{~mm}$ (Linke et al., 2006). The plate is heated up to a temperature sufficient for inducing the Leidenfrost effect. A drop which is placed on top of this surface immediately starts moving with comparatively large speed perpendicular to the steps. The drop thereby climbs up the steep side of the step and slides down the flat side which induces directed motion of the drop. This behavior could be observed for various liquids with different boiling points (Linke et al., 2006; Quere and Ajdari, 2006). This work attracted great attention also because this setting was suggested as an energy efficient cooling device which uses the waste heat energy as energy source for driving the refrigerant. The most severe challenge to this approach is that the heat conductivity between the surface which should be cooled and the cooling fluid is strongly reduced due to the Leidenfrost effect, which makes it intrinsically difficult to transfer heat to the refrigerant. This is why the Leidenfrost effect is usually unfavorable in industrial cooling devices. Nevertheless, it is an interesting application of the Leidenfrost effect.

Another application of the Leidenfrost effect is investigated in Elbahri et al. (2007) and Bain (2007). A droplet of water which is experiencing the Leidenfrost effect contains small nano-particles of metal (silver or zinc oxide) and is hovering above a silicon surface. While the droplet sits on the surface it deposits a small amount of solid material. As the droplet starts moving it leaves a trail, a nanowire behind and therefore is a potentially useful technique in nanotechnology. 
and usually has, periodic boundary conditions. Due to the large mass of individual granular particles, it is not sufficient to place the container onto a hot surface. This would heat up the grains but would not accelerate the particles themselves, implying that the role of the hot surface, which in the classical setup with water is a heat bath accelerating particles, is taken by a vertically vibrating container wall.

Strong shaking of dry grains under gravity leads to a granular gas where one finds a monotonically decreasing density with increasing height which is reminiscent of the barometric formula. However, in the case of granular matter the solution is not unique due to the energy dissipation (Eggers, 1999). Moreover, if, at fixed shaking strength, the number of particle layers is increased, a density inversion sets in (Huan et al., 2004; Brey et al., 2001; Kudrolli et al., 1997; Lan and Rosato, 1995; Poeschel and Schwager, 2004; Taberlet et al., 2007; Eshuis et al., 2007). Therefore, the density of particles is not decreasing with increasing distance from the bottom container wall, but it is increasing and therefore the density in this state is lowest directly at the bottom container wall. This phenomenon was observed in Molecular Dynamics (MD) simulations and theoretically described in Meerson et al. (2003) and Poeschel and Schwager (2004) for dry grains interacting through inelastic collisions. A snapshot of such a simulation is shown in Fig.6.2. The first experiment which was carried out in a quasi two-dimensional setup was presented by Eshuis et al. (2005). In this work, the effect of the observed density inversion in granulates was called the granular Leidenfrost effect for the very first time. The experiments were also accompanied by a theory of granular hydrodynamics which reproduces the experimentally observed density profiles. The difference to the earlier studied density inversion (Meerson et al., 2003) is that they only speak of a Leidenfrost state if there is really an observable phase separation into a dense solid phase (hexagonally packed for monodisperse particles) hovering above a dilute gas phase. They also introduced an order parameter for detecting the respective phase, based on the crystalline order. A phase diagram for dry granular matter was presented in Eshuis et al. (2007). This shows that the Leidenfrost effect can clearly be distinguished from a state where only a bouncing bed or convection is present and can only be observed when the filling height exceeds a critical number of about eight particle layers and the driving energy of the wall is sufficiently large.

\subsection{Leidenfrost effect in wet granular matter}

In contrast to dry granular material, where the Leidenfrost effect is a well studied phenomenon, we investigate this effect in wet granular matter where we find remarkable new features. Therefore, we perform MD type simulations with ideally spherical, frictionless particles under gravity in two and three dimensions. In the simulations the thin-thread model for the capillary interaction of the capillary bridge as introduced in Sec. 2.3 is used and, through the event-driven simulation technique which can be applied here, collisions between spheres are modeled by a 


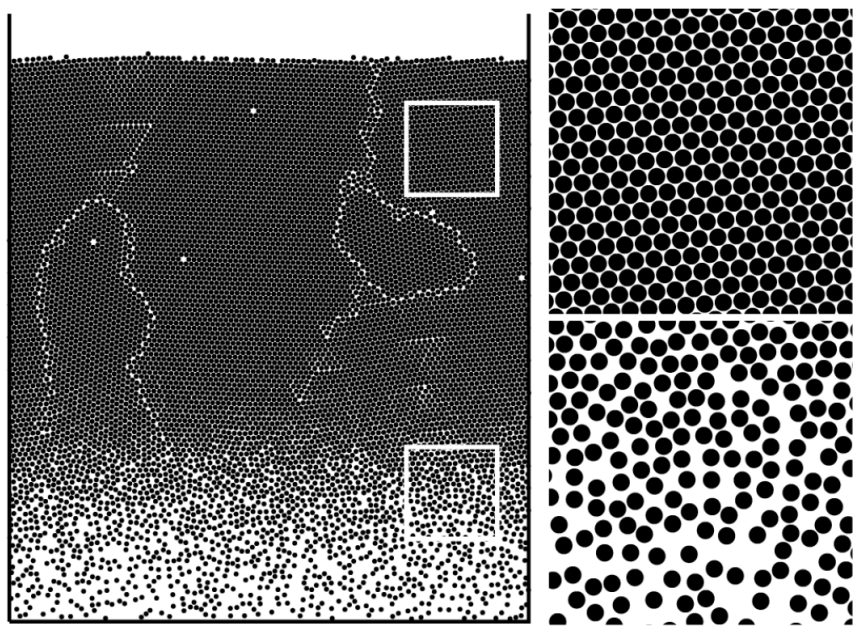

Fig. 6.2 Simulation of $10^{4}$ two-dimensional, dry, inelastic particles (coefficient of restitution $\varepsilon=$ 0.98815). The hexagonally closed packed solid plug is hovering above a gaseous region. Because the monodisperse particles are confined in a container there are even some defect lines visible in the crystallized part. The highlighted areas (white square boxes) are magnified on the right hand side of the figure. Picture reprinted with permission from (Meerson et al., 2003). Copyright (2003) by the American Physical Society (dx.doi.org/10.1103/PhysRevLett.91.024301).

hard core potential. The collision itself is assumed to be elastic unless stated otherwise. The grains are put into a container and shaken vertically with a sinusoidal driving function. All simulations and results shown in this chapter are produced with this simulation technique. We additionally performed few time-driven simulations using the minimal capillary model (as introduced in Sec.2.3) to verify that the observed effects are not an artifact of the interaction model for the capillary bridge.

If the driving is sufficiently strong (e.g. $E^{*}=98.9$ and $\Gamma=60.0$ ), we obtain in the simulation a state as shown in Fig.6.3a. We observe a cold, solid plug hovering on the hot, dilute gas which is analogous to the earlier described Leidenfrost phenomenon. The color of each particle denotes its individual kinetic energy $E_{\text {kin }}$ on a logarithmic scale (see inset) normalized with respect to the bridge energy $E_{\mathrm{cb}}$, where blue (red) means low (high) kinetic energy. It is noted that the kinetic energies vary by five orders of magnitude within the sample. The snapshots were taken at different times during the simulation. Obviously, the plug is not at rest but is moving vertically. This behavior is examined in the next subsection.

\subsection{Vertical plug oscillation}

As seen in Fig.6.3a, we observe a vertical movement of the dense plug hovering on a hot gas. To examine this behavior in detail, we look at the areal density of the sample. From the snapshots it is obvious that we can average over the horizontal spatial dimensions to improve the quality of the data. This areal density, spatially 


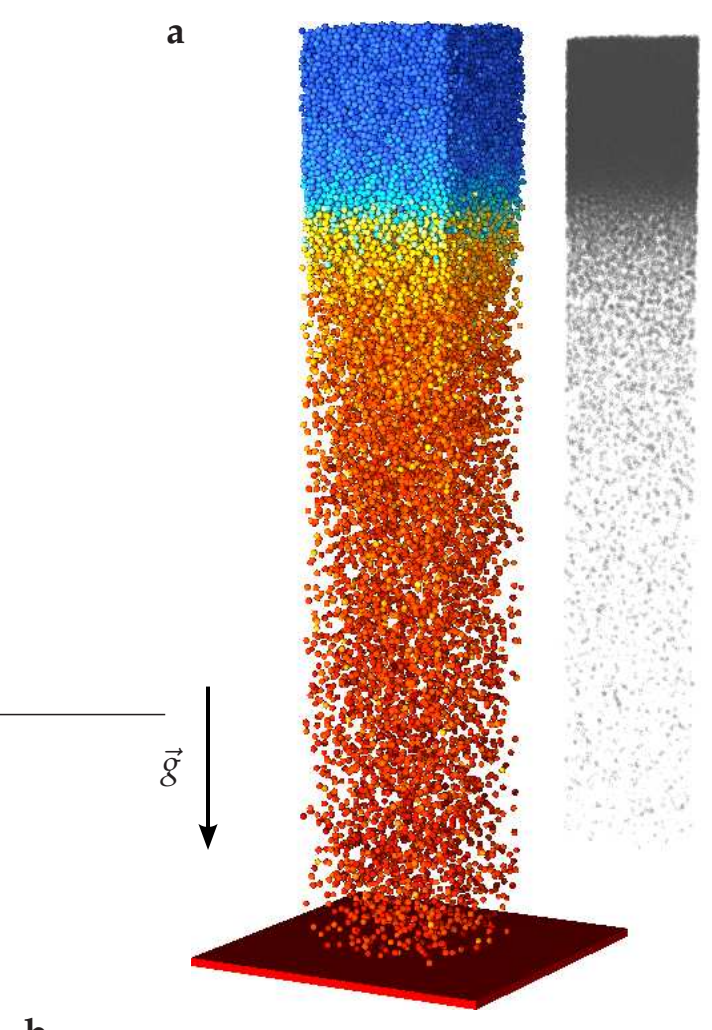

b
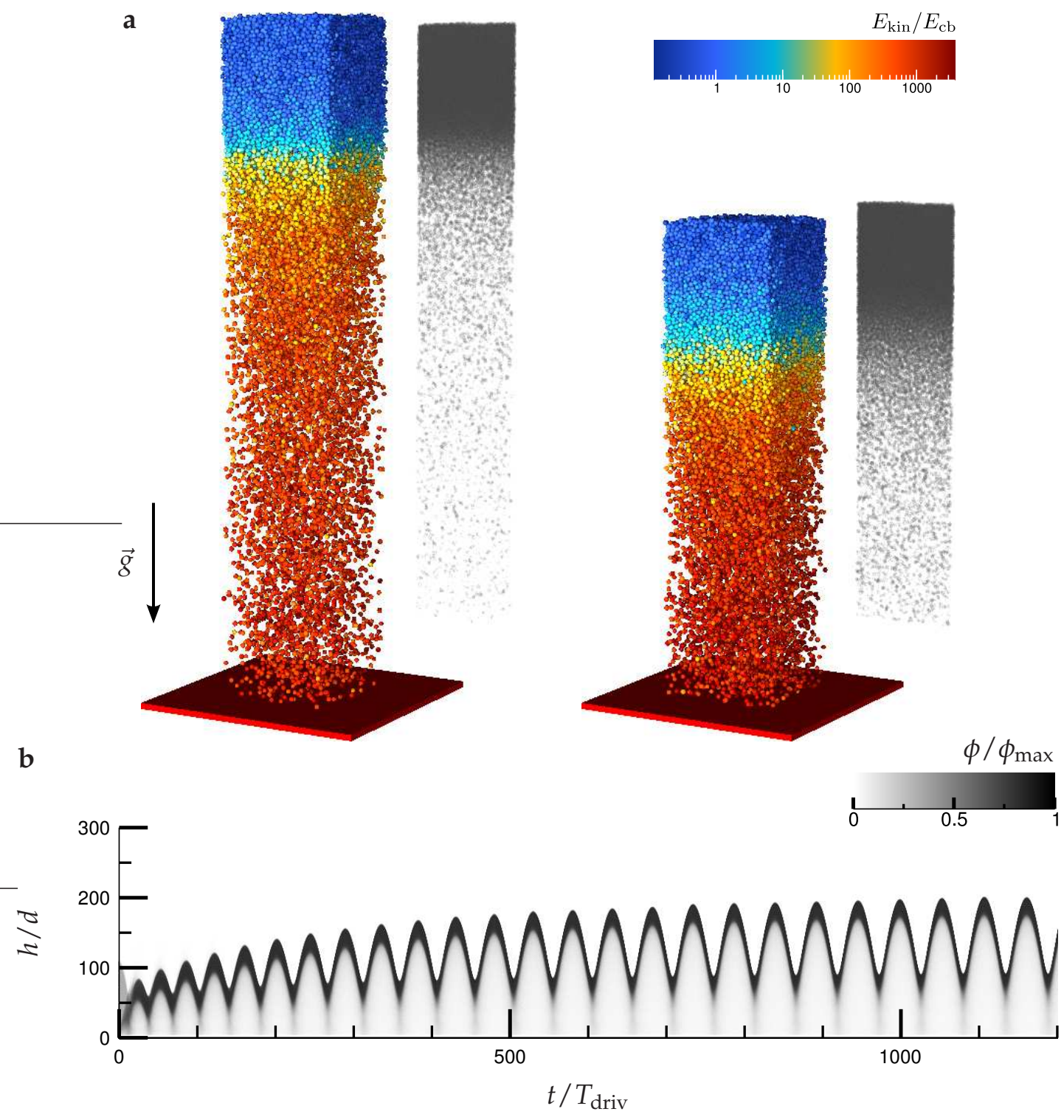

Fig. 6.3 a Snapshots at different times of a three-dimensional simulation for $3 \cdot 10^{4}$ slightly polydisperse particles showing the Leidenfrost state. Gravity $g$ is pointing downwards and the box has a square base with each edge being 25 particle diameters in length, so the particles form 48 layers at rest. The box has periodic boundary conditions in both directions perpendicular to gravity. The color indicates the kinetic energy of each particle on a logarithmic scale. The shadow is generated digitally in order to account for the areal density. $\mathbf{b}$ The Areal density $\phi$ (averaged over lateral dimensions) is shown as gray value normalized with respect to the maximum packing fraction $\phi_{\max }$ as shown by the scale bar (white means no particles and black denotes the maximum density). It is shown as a function of the height $h$ in the system normalized with respect to the diameter $d$ of one particle and the time $t$ which is normalized with respect to the periodic time $T_{\text {driv }}$ of the driving. The plug oscillation is clearly visible. The mean force induced by the capillary bridge is $F_{\mathrm{cb}} / F_{g}=4$, where $F_{g}$ is the gravitational force. The capillary bridge ruptures at a surface separation of $s_{\text {crit }} / d=0.0711$. The parameters of the driving are $E^{*}=98.9$ and $\Gamma=60.0$. 
depending only on the height $h$ above the bottom plate, is shown in Fig. $6.3 \mathrm{~b}$ as a function of the time $t$ as a grayscale image where white (black) means low (high) density. It is normalized with respect to the maximum packing fraction $\phi_{\max }$ which is 0.74 in three dimensions, the height is normalized with respect to the diameter of one particle $d$ and the time is normalized with respect to the time period $T_{\text {driv }}$ of the driving function.

At the beginning of the simulation at $t=0$ the particles are inserted close to the bottom of the container and are regularly ordered on a simple cubic lattice. It can be seen in Fig. $6.3 \mathrm{~b}$ that the Leidenfrost state very rapidly evolves out of this state. Afterwards, a vertical oscillation of the plug is clearly visible which in the end of the simulation becomes stationary with a constant amplitude and frequency. The system was actually simulated more than five times longer than shown here.

Now we look at the density profile which we obtain out of Fig. $6.3 \mathrm{~b}$ for a constant time. To improve statistics, we do an average of multiple measurements taken at different times. We perform these measurements at a constant phase to both the driving frequency and the plug oscillation frequency. In Fig. 6.4 the density profile is shown for the top and bottom turning point of the plug oscillation, respectively. The density in the plug is approximately 0.62 , which is close to the packing fraction of approximately 0.64 obtained by random close packing in three dimensions (Torquato et al., 2000; Martin and Bordia, 2008). The phase boundary between the gas and the solid plug can be clearly identified. This is in contrast to dry granular matter where no pronounced phase boundary is visible. For comparison, Fig. 6.5 shows the density profile for dry granular matter.

If the driving energy of the oscillating wall is lowered to $E^{*}=63.3$, we still observe the Leidenfrost effect, but the oscillation of the plug becomes more irregular. This is illustrated in Fig.6.6, where we again see the height dependent areal density plotted versus time. In the top part of the image we clearly observe that the amplitude of the oscillation is modulated by an enveloping function with a much lower frequency than the plug oscillation itself. In the bottom part of the figure, the same image is shown again with lower contrast, so that individual particles become visible. We observe that the modulation of the amplitude is accompanied by a dilute gas above the plug, which is not present in the simulation of Fig.6.3. We can see that the plug in Fig. 6.6 comes closer to the bottom during its oscillation and therefore the reduction of heat conductivity becomes less effective and some particles at the top obtain a higher kinetic energy as the particles in the bulk. This means that for even lower driving energies $E^{*}$ the amount of gas above the dense plug is increasing, as confirmed by simulations.

The Leidenfrost point is, in the classical setup with water, the temperature of the hot surface at which the floating drop reaches its longest lifetime (Walker, 1988). Because the system in the granular setup is closed and particles cannot vanish, it is not possible to measure a lifetime of the drop directly. Nevertheless, we can imagine the system to be open, such that all particles are removed which run through the periodic boundary conditions. The loss of particles is then mainly driven by the 


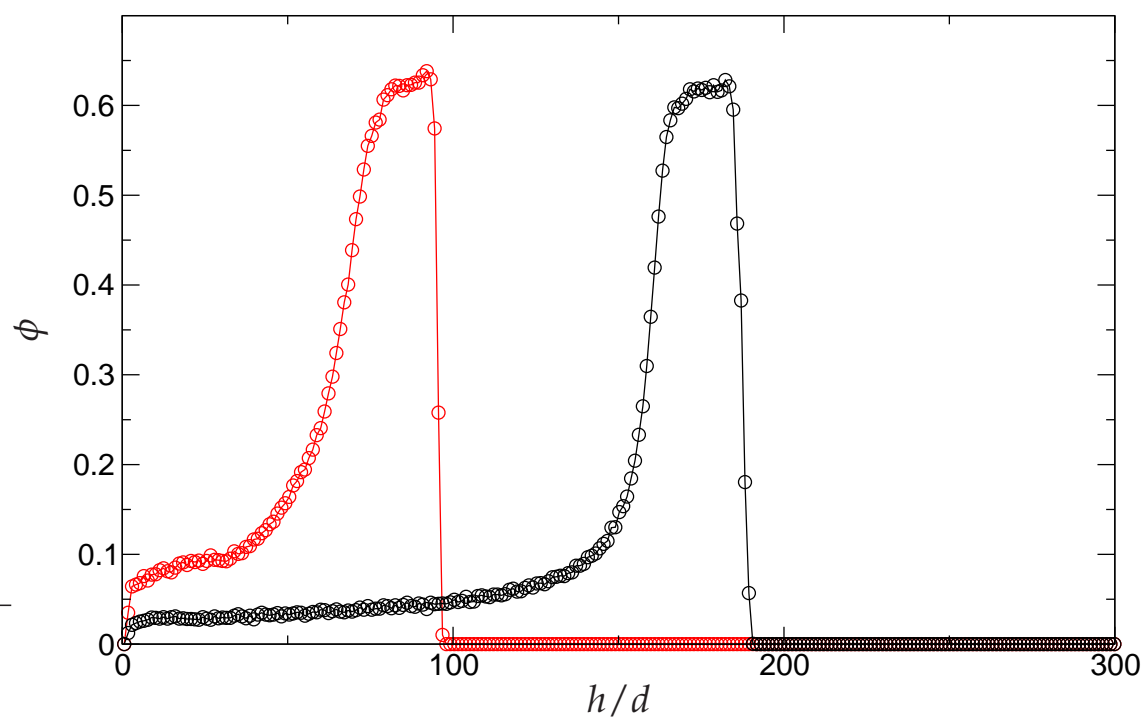

Fig. 6.4 Areal density plotted versus the height in the container. The plug oscillation is in its top (black) and bottom (red) turning point, respectively. It is averaged over eight consecutive cross sections taken out of Fig.6.3b). The dense plug at the top reaches a density of about $\phi=0.62$, which is almost random close packing. An interface between the two regions can easily be defined and therefore it is justified to speak of a condensed plug hovering on a gas.

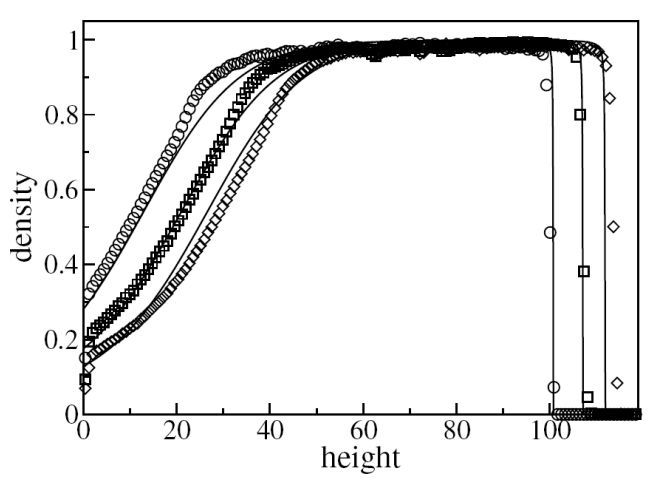

Fig. 6.5 Density profile for dry granular matter predicted by hydrodynamics (solid lines) and simulated in MD simulations (symbols) for different parameters. No clear boundary between the two phases is visible. In fact it is a continuous increase of the areal density with height. For more information see (Meerson et al., 2003). Reprinted with permission from (Meerson et al., 2003). Copyright (2003) by the American Physical Society (dx.doi.org/10.1103/PhysRevLett.91.024301). 


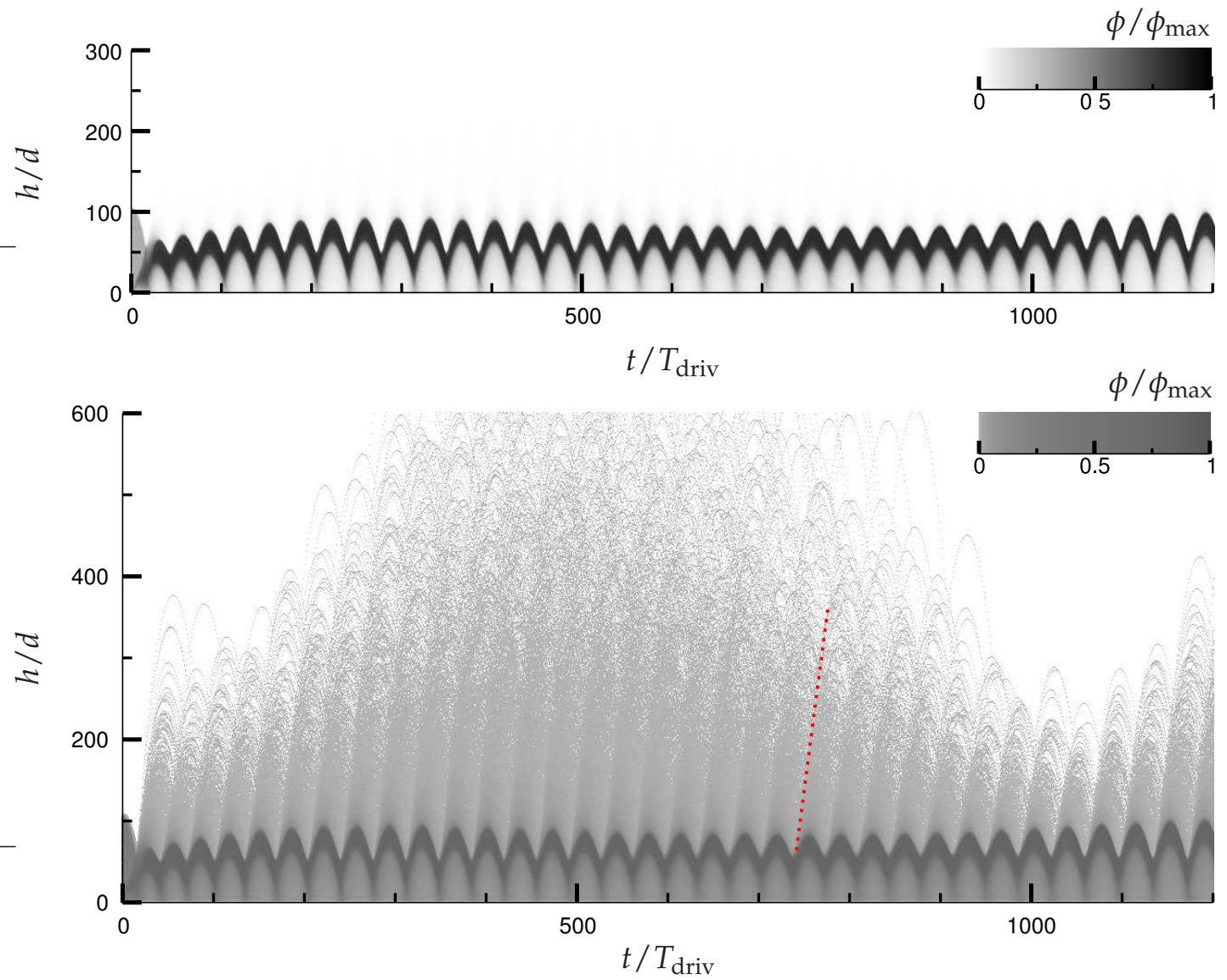

Fig. 6.6 top: Areal density (gray value) plotted as a function of height and time. The Leidenfrost effect as well as the vertical plug oscillation is visible. The amplitude of the vertical oscillation, however, is modulated periodically. The system is simulated more than six times longer than shown in the graph, and this modulation is present all over. bottom: Same image as above but with lower contrast, so that the dilute gas above the plug becomes visible and even tracking of individual particles is possible. The actual height of the box is higher than shown here, and no particle collided with the upper wall of the simulation box. The dotted line (red) denotes the velocity of sound which is similar in the order of magnitude to the mean speed of the granular gas above the plug (Chapman and Cowling, 1970, pp. 36). The velocity of sound, $v_{\text {sound }}$, as deduced from simulations is $v_{\text {sound }} \frac{T_{\text {driv }}}{d} \approx 11.3$. The simulation was performed at driving parameters $E^{*}=63.3$ and $\Gamma=60.0$. 
granular gas (above and below the plug), because of its high kinetic energy. This means, that the higher the kinetic energy of the gas and the larger its contact area with the system borders (which show the periodic boundary conditions), the faster the particles are removed from the system. Therefore, the longest lifetime, which defines the Leidenfrost point we are searching for, is associated with the system being in a state with no gas layer above and the smallest possible gas layer below the plug. This situation reduces the contact area with the system borders and the kinetic energy of the gas layer below the plug. The value of the driving energy $E^{*}$, at which this situation occurs, is the Leidenfrost point we were looking for. Furthermore, we can think of the maximum driving energy $E^{*}$, in the granular setup, as the 'temperature' of the wall and therefore can also regard this value as the analogue to the Leidenfrost temperature. The Leidenfrost point, for the system described above, is $E_{\mathrm{LP}}^{*}=92.4 \pm 6.5$ for $\Gamma=60.0$.

\subsection{Theoretical model}

We now study the vertical oscillation of the plug and put forward a possible mechanism for this phenomenon. We restrict ourselves to the case without any additional gas above the plug.

\subsubsection{Passive plug on gas layer}

We first consider a solid plug of mass $m$ which moves without friction in a tube of cross section $A_{g}$ above a cushion of ideal gas with height $h$ and pressure $p=$ $N k_{B} T / A_{g} h$. This is shown in Fig.6.7. By $N$ we denote the total number of particles in the gas column, $k_{B}=1$ is 'Boltzmann's constant' and we assume for the moment that the whole system is at temperature $T_{g}$ without any temperature gradient. The equation of motion reads

$$
m \frac{\mathrm{d}^{2} h}{\mathrm{~d} t^{2}}=p A_{g}-m g=\frac{N k_{B} T}{h}-m g
$$

where $g$ is the acceleration due to gravity and $t$ is time. Stationarity yields the equilibrium height, $h_{0}=N k_{B} T / m g$. Small deviations from $h_{0}$ shall be described by $h(t)=h_{0}\left(1+\varepsilon_{h}(t)\right)$, with $\varepsilon_{h} \ll 1$. Inserting this into Eq. (6.I) leads to

$$
\frac{\mathrm{d}^{2} \varepsilon_{h}}{\mathrm{~d} t^{2}}=-\frac{g}{h_{0}} \varepsilon_{h}
$$

for isothermal excursions, and to

$$
\frac{\mathrm{d}^{2} \varepsilon_{h}}{\mathrm{~d} t^{2}}=-\kappa \frac{g}{h_{0}} \varepsilon_{h}
$$

for adiabatic (i. e., sufficiently rapid) excursions from $h_{0}$, where $\kappa=5 / 3$ is the adiabatic index for three-dimensional, frictionless and elastic particles. In the isothermal 
and adiabatic case, the result is purely oscillatory, with frequency $\omega_{p}=\sqrt{g / h_{0}}$ and $\omega_{p}=\sqrt{\kappa g / h_{0}}$, respectively. We restrict the discussion to the adiabatic case, which appears appropriate for the 'experimental' situation.

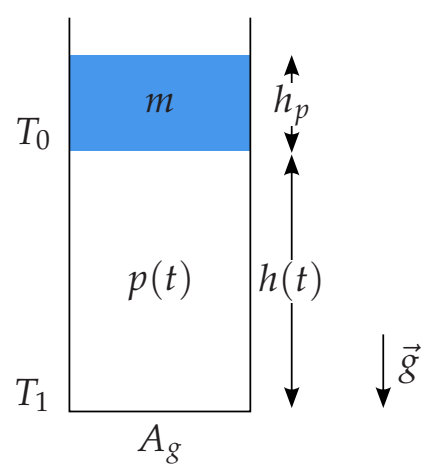

Fig. 6.7 Solid plug of mass $m$ is hovering on an ideal gas cushion of height $h$ with pressure $p$ in a tube of cross section $A_{g}$. Gravity $\vec{g}$ is pointing downwards. Additionally, in section 6.4 .3 the temperature of the bottom will be $T_{1}$ and the plug at temperature $T_{0}$ with $T_{0}<T_{1}$.

Because the particles in the granular gas do have a certain spatial extent, we can also include this via the known Van der Waals equation $p\left(A_{g} h-V_{\mathrm{ex}}\right)^{\kappa}=$ const. with $V_{\text {ex }}=4 N V_{\text {bead }}$ being the usual total excluded volume ${ }^{2}$ of spherical particles with volume $V_{\text {bead }}$. The correction of the pressure in the Van der Waals equation which is due to attractive forces between particles is negligible in this case, because attractive forces arise in the system solely through the capillary bridges and we have seen in Fig. 6.3 that the kinetic energy of the particles in the gas is much larger than the capillary bridge energy. The equation of motion in this case reads

$$
\frac{\mathrm{d}^{2} \varepsilon_{h}}{\mathrm{~d} t^{2}}=-\kappa \frac{g}{h_{0}}\left(1+\frac{V_{\mathrm{ex}}}{V}\right) \varepsilon_{h}
$$

with the oscillation frequency

$$
\omega_{p}=\sqrt{\kappa \frac{g}{h_{0}}\left(1+\frac{V_{\mathrm{ex}}}{A_{g} h_{0}}\right)} .
$$

In what follows, we will assume the oscillations of the plug as being fast compared to the thermal exchange with the wall and the plug, such that the process is adiabatic. It is worth noting that the oscillation frequency of the plug does not depend explicitly on its mass ${ }^{3}$, neither in the isothermal or adiabatic case of an ideal gas nor in the adiabatic case of a real gas which is corrected by the excluded volume of spherical particles.

2. The factor four in the excluded volume results from the volume $\frac{4}{3} \pi(2 R)^{3}=4 \times 2 V_{\text {bead }}$ containing two spheres with volume $V_{\text {bead }}=\frac{4}{3} \pi R^{3}$.

3. There is an implicit dependence of the oscillation frequency on the mass of the plug because the height of the gas cushion depends on the mass of the plug $h_{0}(m)$. 


\subsubsection{Plug on a gas layer with exchange of particles}

We now assume that the plug consists only of the condensate of the gas below it and therefore allow interaction between the plug and the gas, in contrast to the passive plug discussed above. It is assumed that the plug and the gas are in equilibrium with each other, such that there is a continuous exchange of particles between them. The rate of exchange can be quantified by introducing an exchange frequency $v$, defined as the inverse of the time it would take for the entire gas to condense at the bottom of the plug if any escape from the plug was prevented (unity sticking coefficient). In the dilute limit, the gas would condense ballistically on the plug surface. We thus have $v_{\text {dilute }} \approx c / h_{0}$ in this case, where $c$ is the typical velocity of the particles. In the dense limit, the condensation of the gas would proceed diffusively, and we obtain $v_{\text {dense }}=D / h_{0}^{2}$, with the diffusivity $D$. Since $D$ is given by the average particle velocity times their mean free path, $\xi_{p}$, this can be written as $v_{\text {dense }}=v_{\text {dilute }} \xi_{p} / h_{0}$.

If the pressure under the plug deviates from its equilibrium value, there will be a net flow between the plug and the gas, given by

$$
\frac{\mathrm{d} n}{\mathrm{~d} t}=v n_{0} \frac{p_{0}-p}{p_{0}}
$$

where $n:=N / A_{g}$ and $n_{0}$ is the equilibrium gas density. If we write $n(t)=n_{0}(1+$ $\delta(t))$, it follows that

$$
p(t) \approx \frac{n_{0} k_{B} T}{A_{g}^{\kappa-1} h_{0}^{\kappa}}(1+\delta(t))\left(1-\kappa \varepsilon_{h}(t)\right) \approx p_{0}\left(1+\delta(t)-\kappa \varepsilon_{h}(t)\right) .
$$

Inserting this into eqs. (6.1) and (6.6), we directly obtain

$$
\begin{aligned}
\frac{\partial \delta}{\partial t} & =-v\left(\delta-\kappa \varepsilon_{h}\right) \\
\frac{\partial^{2} \varepsilon_{h}}{\partial t^{2}} & =\frac{\omega_{p}^{2}}{\kappa}\left(\delta-\kappa \varepsilon_{h}\right) .
\end{aligned}
$$

With the exponential ansatz $\delta(t)=\delta_{0} \exp (\lambda t)$ and $\varepsilon_{h}(t)=\varepsilon_{0} \exp (\lambda t)$, this leads to $(\lambda+v) \delta=v \kappa \varepsilon_{h}$ and $\left(\lambda^{2}+\omega_{p}^{2}\right) \varepsilon_{h}=\frac{\omega_{p}^{2}}{\kappa} \delta$. This combines to the characteristic polynomial

$$
\left(\Lambda^{2}+1\right)(\Lambda+B)-B=\Lambda\left(\Lambda^{2}+\Lambda B+1\right)=0
$$

with the abbreviations $\Lambda:=\lambda / \omega_{p}$ and $B:=v / \omega_{p}$. Besides the trivial solution at $\Lambda_{0}=0$, which represents the steady state, we obtain the non-trivial solutions through

$$
\Lambda_{ \pm}=-\frac{B}{2} \pm \sqrt{\left(\frac{B}{2}\right)^{2}-1}
$$


If the non-dimensional exchange frequency of particles between the plug and the gas, $B$, is zero, we obtain $\Lambda_{ \pm}= \pm i$ which is an undamped oscillation. If $B$ is larger than zero, the solution $\Lambda_{ \pm}$always has a negative real part, which indicates damping and therefore leads always to a decay to the steady state. This occurs in the range of $0<B<2$, where the particle exchange frequency, $v$, is small compared to the oscillation frequency of the plug, $\omega_{p}$. For $B \geqslant 2$, which means faster particle exchange, $\Lambda_{ \pm}$is purely real and negative, such that we obtain an exponential decay rate. The special case of $B=2$ is the overdamped solution with $\Lambda_{ \pm}=-1$. Fig.6.8 shows the roots of Eq. (6.10) in the complex plane for $B \in[0,2]$. The two complex conjugate eigenvalues $\Lambda_{ \pm}$correspond to the oscillating mode, with the negative real part indicating a damped motion. As $B$ increases and becomes equal to two, the two complex conjugate solutions merge on the real axis at $\Lambda_{ \pm}=-1$, which is the overdamped motion.

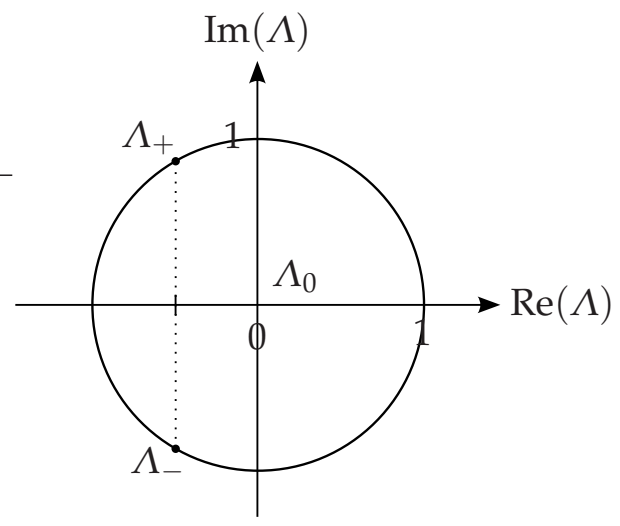

Fig. 6.8 The roots of Eq. (6.10) in the complex plane. Besides the trivial root at $\Lambda_{0}=0$, the non-trivial roots are lying on the unit circle. For $B=0$ which is $\Lambda_{ \pm}= \pm i$ yields undamped oscillation while $B=2$ leads to $\Lambda_{ \pm}=-1$ which is the overdamped solution. $\Lambda_{ \pm}$as marked in the figure is for $B=1$ which is a damped oscillation.

\subsubsection{Plug on a gas layer with exchange of particles and a temperature gradient}

Now we consider the case where the bottom of the gas is being heated to some elevated temperature $T_{1}>T_{e}$, while the plug is assumed to remain, in temporal average, at a temperature $\left(T_{0}\right)$ slightly below its evaporation temperature $T_{e}$ (Biance et al., 2003). This is roughly the situation in the Leidenfrost effect. Cooling of the plug is provided by evaporation of gas from the plug. In the conventional setting involving standard liquids, this evaporation inevitably leads to shrinking of the liquid drop as it hovers on the hot surface. With wet granular matter however, the energy dissipation is intrinsic and can proceed forever in a closed system.

The heat current, $j$, through the gas cushion below the plug is determined from the temperature gradient, $\left(T_{1}-T_{0}\right) / h=: \Delta T / h$, and the thermal conductivity of the gas, $\Theta$. We thus have $j=\frac{\Theta \Delta T}{h} \approx \frac{\Theta \Delta T}{h_{0}}\left(1-\varepsilon_{h}(t)\right)$. We assume that the heat current 
will be balanced by evaporative cooling. If $h$ increases (positive $\varepsilon_{h}$ ), the temperature gradient is reduced, and there is less evaporation, because $\Theta$ is largely independent of density in a gas. As a consequence, more gas will condense on the plug, and the number of particles in the gas cushion is decreased. This can be quantified as

$$
H \frac{\mathrm{d} n}{\mathrm{~d} t}=-\frac{\Theta \Delta T}{h_{0}} \varepsilon_{h}
$$

where $H$ is the energy required for the evaporation of a single particle from the plug. We now can write

$$
\frac{\partial \delta}{\partial t}=v\left(\kappa \varepsilon_{h}-\delta\right)-\theta \varepsilon_{h}
$$

with the abbreviation

$$
\theta=\frac{\Theta \Delta T}{n_{0} h_{0} H} .
$$

This leads to the modified characteristic polynomial

$$
\left(\Lambda^{2}+1\right)(\Lambda+B)-B+A=0
$$

in which

$$
A:=\frac{\Theta \Delta T}{\kappa \omega_{p} n_{0} h_{0} H}
$$

represents the temperature gradient in dimensionless form. We see in Eq. (6.15) directly that the temperature gradient counteracts the damping. The system is in a stationary state if the damping, through the exchange of particles, is balanced by the driving, through the temperature gradient, which is the case at $A=B$. The oscillation then becomes undamped, because $\Lambda= \pm i$ is a root. We are now equipped to understand the vertical oscillations observed in simulations of vertically agitated wet granular matter. The oscillation frequency of the plug in this non-equilibrium steady state is the same as obtained in Eq. (6.5) for the passive plug being in equilibrium. In subsection 6.5.1, we compare the theoretical oscillation frequency with the oscillation frequency obtained in simulations. In these simulations, the lateral extension of the system was very limited, such that the lateral coordinate could be disregarded.

The theory, as presented here, accounts for elastically colliding wet granular particles. The energy of evaporation $H$, which is the energy that has to be spent in order to overcome the attractive forces, is given by the capillary bridge energy and is a constant. In dry granular matter, where there are no attractive forces between particles, it is not obvious that there should be a energy of evaporation as a single constant value. In fact, as mentioned before, it is not even obvious that there is a clear interface between the plug and the gas cushion underneath. Therefore, one has to use a more elaborate model in order to account for dry granular matter. 


\subsubsection{The complete picture, with lateral gas exchange}

The situation becomes more complex if we allow lateral variations of quantities like the gas pressure or density. These must then be considered as functions of both time and a lateral coordinate, $x$. We imagine the system sketched in Fig.6.7 as laterally infinite, but we keep the idea of a finite thickness, $h_{p}$, of the drop (or puddle). The lower surface of the drop is thought to undergo excursions given by $h(x, t)$. We will have to distinguish between excursions at small wavelengths as compared to $h_{p}$, in which case we can assume the upper surface to remain flat, and those with a large wavelength, which will be directly followed by the upper surface which is then not flat anymore. The main difference is that in the former case, the inertial mass of the drop which participates in the motion is given by $m(q) \approx \rho / q$, where $\rho$ is the mass density of the plug and $q$ is the wave number. In the latter case, however, the mass is always equal to $\rho h_{p}$. A suitable interpolation between these two cases is $m(q)=\frac{\rho}{q} \tanh q h_{p}$.

Assuming linearity, we can consider spatially harmonic eigenmodes of the system, with wave number $q$. The equation of motion of this system can then be written as

$$
m(q) h_{0} \frac{\partial^{2} \varepsilon_{h}}{\partial t^{2}}=p_{0}\left(\delta-\kappa \varepsilon_{h}\right)+h_{0}\left(\rho g-\gamma q^{2}\right) \varepsilon_{h} .
$$

The second term on the right hand side contains the usual dispersion of (upside down) capillary waves, with $\gamma$ being the surface tension of the liquid the plug consists of. It is noted that $p_{0}=\rho h_{p} g$ since the total weight of the drop determines the pressure under it. We consider the two important cases for large $q^{-1} \gg h_{p}$ and small wavelengths $q^{-1} \ll h_{p}$. For large wavelengths $2 \pi q^{-1}$, we set $m=\rho h_{p}$ and obtain

$$
\frac{\partial^{2} \varepsilon_{h}}{\partial t^{2}}=\frac{\omega_{p}^{2}}{\kappa}\left(\delta-\kappa \varepsilon_{h}\right)+\omega_{1}^{2}\left(1-(q l)^{2}\right) \varepsilon_{h}
$$

with $\omega_{1}:=\sqrt{g / h_{p}}$, and $l=\sqrt{\gamma / \rho g}$ is the capillary length of the liquid. It is noted that $\omega_{p}^{2} / \omega_{1}^{2}=h_{p} / h_{0} \ll 1$. For small wavelengths $2 \pi q^{-1}$, we set $m=\rho / q$ and find that the right hand side of Eq. (6.18) is just multiplied by $q h_{p}$. We will see below that this case is of minor importance for the present study.

In the case of the density variation $\delta$, we introduce a lateral flow conductivity of the gas layer as $\mu:=h_{0}^{2} / 12 \eta$, where $\eta$ is the viscosity of the gas (note that this holds only if $q h_{0} \ll 1$ ). This leads to an additional contribution to the local change of gas density, as described by

$$
\frac{\partial \delta}{\partial t}=\mu \frac{\partial^{2} p}{\partial x^{2}}=\mu \frac{\partial^{2} p_{0}\left(\delta-\kappa \varepsilon_{h}\right)}{\partial x^{2}}
$$

Invoking the harmonic ansatz again, we can write, in analogy with Eq. (6.13),

$$
\frac{\partial \delta}{\partial t}=\left(\nu+\beta Q^{2}\right)\left(\kappa \varepsilon_{h}-\delta\right)-\theta \varepsilon_{h}
$$


with $\beta:=p_{0} h_{0}^{2} / 12 \eta l^{2}$ and $Q:=q l$. Combining Eqs. (6.18) and (6.20), we arrive at

$$
\left(\lambda^{2}+\omega_{p}^{2}-\omega_{1}^{2}\left(1-Q^{2}\right)\right)\left(\lambda+v+\beta Q^{2}\right)=\frac{\omega_{p}^{2}}{\kappa}\left(\left(\nu+\beta Q^{2}\right) \kappa-\theta\right) .
$$

With the definitions $\Omega^{2}:=\omega_{p}^{2}-\omega_{1}^{2}\left(1-Q^{2}\right), \Lambda:=\lambda / \Omega$, and the redefinition of $B:=\left(v+\beta Q^{2}\right) / \Omega$, we obtain the characteristic polynomial

$$
\left(\Lambda^{2}+1\right)(\Lambda+B)+\frac{\omega_{p}^{2}}{\Omega^{2}}\left(\frac{\theta}{\kappa \Omega}-B\right)=0
$$

We see that the critical temperature gradient, $\theta_{c}$, at which the oscillatory modes, $\Lambda_{ \pm}= \pm i$, become unstable is given by

$$
\theta_{c}=\kappa B \Omega=\kappa\left(\nu+\beta Q^{2}\right) .
$$

This directly shows that the most unstable mode is always the one at $Q=0$, or at the smallest possible $Q$ in a system of finite extension. If the lateral system size (plug diameter) is $L$, the most unstable mode will be at $Q \approx 2 \pi l / L$.

More insight into the behavior of the roots of Eq. $(6.22)$ is gained if it is written as

$$
\Lambda\left(\Lambda^{2}+\Lambda B+1\right)=C
$$

where $C:=\alpha B-\frac{\theta}{\kappa \Omega}(1+\alpha)$, with

$$
\alpha:=\frac{1}{\frac{\kappa h_{p}}{h_{0}\left(1-Q^{2}\right)}-1} \approx\left\{\begin{array}{r}
-\left(1+\frac{\kappa h_{p}}{h_{0}\left(1-Q^{2}\right)}\right)<-1 \text { for } \frac{h_{p}}{h_{0}} \ll \frac{1-Q^{2}}{\kappa} \\
\frac{h_{0}\left(1-Q^{2}\right)}{\kappa h_{p}}>0 \text { for } \frac{h_{0}}{h_{p}} \ll \frac{\kappa}{1-Q^{2}} .
\end{array}\right.
$$

If $C>0$ we find an instability which we identify as the Rayleigh-Taylor instability (Biance et al., 2003) and we find the critical temperature gradient, $\theta_{\mathrm{RT}}$, for this to occur

$$
\theta_{\mathrm{RT}}=\frac{\alpha}{1+\alpha} \theta_{c}
$$

Eq. (6.26) is plotted in Fig. 6.9 and since the setup is heated from below, $\theta$ has to be a positive number by definition, which is why only the positive half of the $\theta$ axis is shown. The solid red curves denote the critical temperature gradient, $\theta_{\mathrm{RT}}$, while the gray shaded areas denote the regions where the Rayleigh-Taylor instability can occur. We have to distinguish the two cases $\alpha>0$ and $\alpha<-1$, which exhibit different physical behavior. Assuming a certain mode $Q$, they can be viewed as the two different approximations $h_{p} / h_{0} \gg 1$ and $h_{p} / h_{0} \ll 1$, i.e. the approximation of small and large height of the vapor cushion $h_{0}$, respectively, compared to the height of the plug $h_{p}$.

First we look at $\alpha>0$. For the root labeled $\Lambda_{0}$ in Fig.6.8, we can safely neglect $\Lambda^{2}$ and obtain $\Lambda_{0} \approx C$. For vanishing $\theta$ (i.e., vanishing temperature gradient), we see 


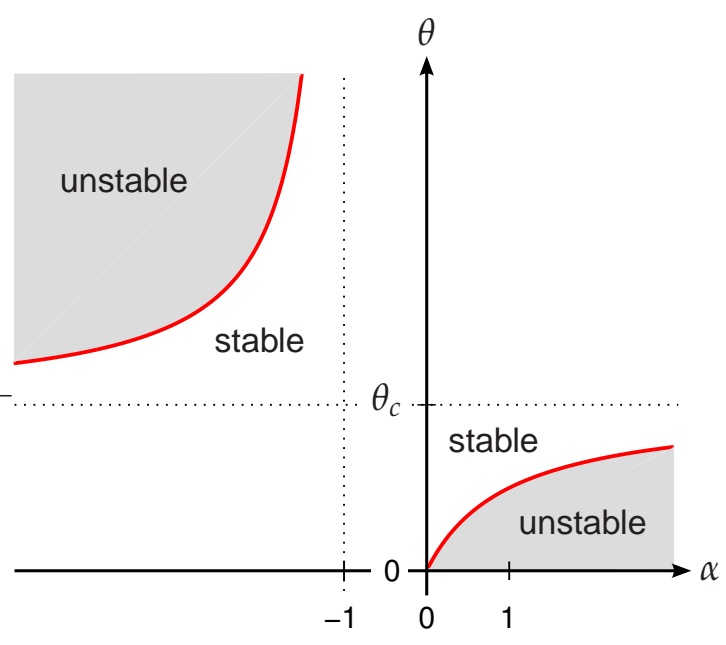

Fig. 6.9 Critical temperature gradient for appearance of the Rayleigh-Taylor instability $\theta_{\mathrm{RT}}$ Eq. (6.26) (red line) plotted versus $\alpha$. The gray shaded areas denote the regions where the Rayleigh-Taylor instability occurs while $\theta_{c}$ is the critical value for the oscillation instability to set in.

that since $C=\alpha B$ is positive, there is always an unstable mode at zero frequency. Interestingly, this mode is stabilized as the temperature gradient is introduced, and is no longer unstable if $C \leqslant 0$, i.e., if $\theta \geqslant \theta_{\mathrm{RT}}$. This is reached (long) before the oscillatory instability discussed above sets in at $\theta_{c}$.

Now we consider $\alpha<-1$. For vanishing $\theta$, we see that since $C=\alpha B$ is negative, there is always a stable mode at zero frequency. This mode is destabilized as the temperature gradient is introduced, and is no longer stable if $\theta \geqslant \theta_{\mathrm{RT}}$. This occurs in contrast to the previous case, after the oscillatory instability sets in at $\theta_{c}$.

As we have seen, a Rayleigh-Taylor instability occurs for $C>0$, which directly led to Eq. (6.26). The equation, as written there, gives an intuitive picture about the connection of both instabilities, the oscillatory instability and the Rayleigh-Taylor instability. However, for the comparison with simulations this formulation is not very convenient and we therefore rewrite Eq. (6.26) for a container of finite size and the most unstable mode ${ }^{4}$ as

$$
L_{c}\left(h_{p}\right)=\sqrt{2} \pi l \sqrt{\frac{h_{0}(v-\beta)+\sqrt{h_{0}\left(-4 h_{p} \beta \theta+h_{0}(\beta+v)^{2}\right)}}{h_{0} v-h_{p} \theta}}
$$

with the critical parameter being the width of the container, $L_{c}$, instead of the temperature gradient, $\theta_{\mathrm{RT}}$, and consider it as a function of the height of the plug, $h_{p}$. These are quantities which are more easily accessible in the simulation. If we restrict ourselves to small values of $h_{p}$, we can perform a series expansion of Eq. (6.27) and

4. $Q \approx 2 \pi l / L$ 
obtain

$$
L_{c}\left(h_{p}\right)=2 \pi l+\frac{\pi l \theta}{h_{0}(\beta+v)} h_{p}+O\left(h_{p}^{2}\right)
$$

which is used later for the comparison of simulation and theory.

What remains to be done is to estimate reasonable numbers for the constants involved in the above calculations and to evaluate whether there is a range of parameters where the instability can be observed. In order to do this, we first see that the heat conductivity of the gas can be written as the diffusivity $D$ multiplied by the specific heat. If we assume the gas to be ideal, we obtain $\Theta=3 D n_{0} k_{B} / 2 h_{0}$. According to Eq. (6.23), this simplifies to the most unstable mode at $Q=0$ to

$$
\Delta T_{c}=\frac{2 \kappa H}{3 k_{B}}
$$

where $H$ is the enthalpy of vaporization.

For a granular system, where we are in the regime of $\alpha<-1$, which is a large vapor cushion compared to the height of the plug, Eq. (6.29) reads

$$
\Delta T_{c}=\frac{10}{9} Z_{b} E_{\mathrm{cb}}
$$

where $Z_{b}$ is the typical coordination number of particles at the plug interface, $E_{\mathrm{cb}}$ is the energy required to rupture a capillary bridge and $k_{B}=1$.

Since enough energy has to be induced to the system to reach the liquid-gas coexistence, we know beforehand that the temperature of the gas will be much larger than $E_{\mathrm{cb}}$ (Fingerle et al., 2008). Hence, the granular system is always in the regime of the oscillatory instability, provided it is hot enough to exhibit a Leidenfrost phenomenon with a sufficiently large vapor cushion. Furthermore, the Rayleigh-Taylor instability should always be accessible by increasing the temperature gradient.

\subsection{Comparison of theory and simulations}

\subsubsection{Plug oscillation frequency}

We compare the theoretical prediction of the plug oscillation frequency $\omega_{p}$ of Eq. (6.5) with the simulations. We therefore perform three-dimensional simulations with $3 \times$ $10^{4}$ elastic particles at $\Gamma=60.0$ for different driving energies $E^{*}$. We measure the height $h_{I}$ of the interface between the gas and the dense plug to obtain the average height $h_{0}$ of the gas layer and we measure the periodic time $T_{p}$ of the plug oscillation to obtain the oscillation frequency $w_{p}=2 \pi / T_{p}$. In Fig.6.10 an areal density function is shown where we see a typical analysis. The red dots represent the position $h_{I}$ of the phase boundary between the gas and the plug for which we assume a critical density of $\phi=0.51$. Variations of this value (in a still reasonable range) do not affect the result significantly. The green and blue dashes denote the detected times 
of the top and bottom turning points, respectively. They provide the period of the plug oscillation. Since we do not have an analytical expression for the number of particles in the gas phase, it is measured in the simulation by counting the number of particles below $h_{I}$.

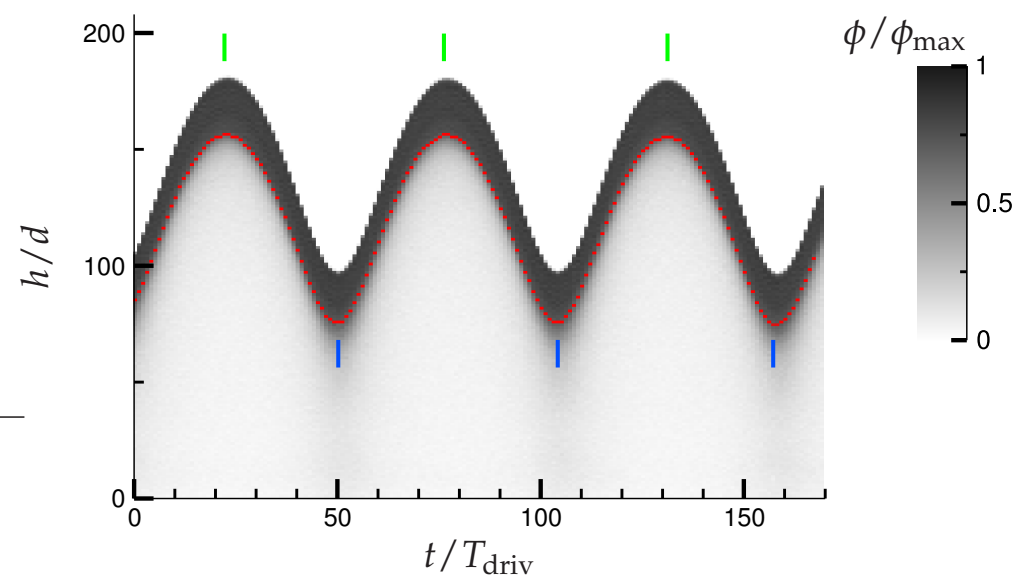

Fig. 6.10 Typical example for the areal density (gray value) of a simulation plotted as a function of height and time. The red points denote the height $h_{I}$ of the interface between the plug and the gas, which was detected by the software and the interface was assumed to be at a density of $\phi=0.51$. The solid vertical dashes denote the time instant where a top (green) and bottom (blue) turning point was detected, respectively. This data provides the period of the oscillation.

Now we plot the acquired data in Fig. $6.11^{5}$ in non-dimensionalized form. The open circles are simulations with different driving energies as denoted in the legend. The solid black line is the theoretical prediction of Eq. (6.5) and we find good agreement with the simulation.

For smaller driving energies $E^{*}$ we get - as discussed earlier - a state with some additional gas above the plug which imposes some additional pressure on the plug. Due to the increased force, the oscillation frequency is also increased (not shown in the figure). The influence of inelastic collisions is shown in appendix 6.A and suggests that the plug oscillations are not observable in experiments for reasonable values of the coefficient of restitution and driving energies.

\subsubsection{Bubbling in wet granular matter}

In the classical Leidenfrost setup with water, an instability was observed by Biance et al. (2003) (cf. Fig.6.12a), which was considered to be the Rayleigh-Taylor instability. We observe a similar effect, reminiscent of bubbling, displayed in the subsequent snapshots in Fig.6.12b-d. An initial periodic perturbation of the interface between

5. It is worth noting, that the data used in Fig.6.11 contains the entire simulation, including the state in which the plug oscillation is not yet stationary. The stationary state is seen in the figure (due to small fluctuations) as an accumulation of points, and is most apparent on the right end of the green curve. 


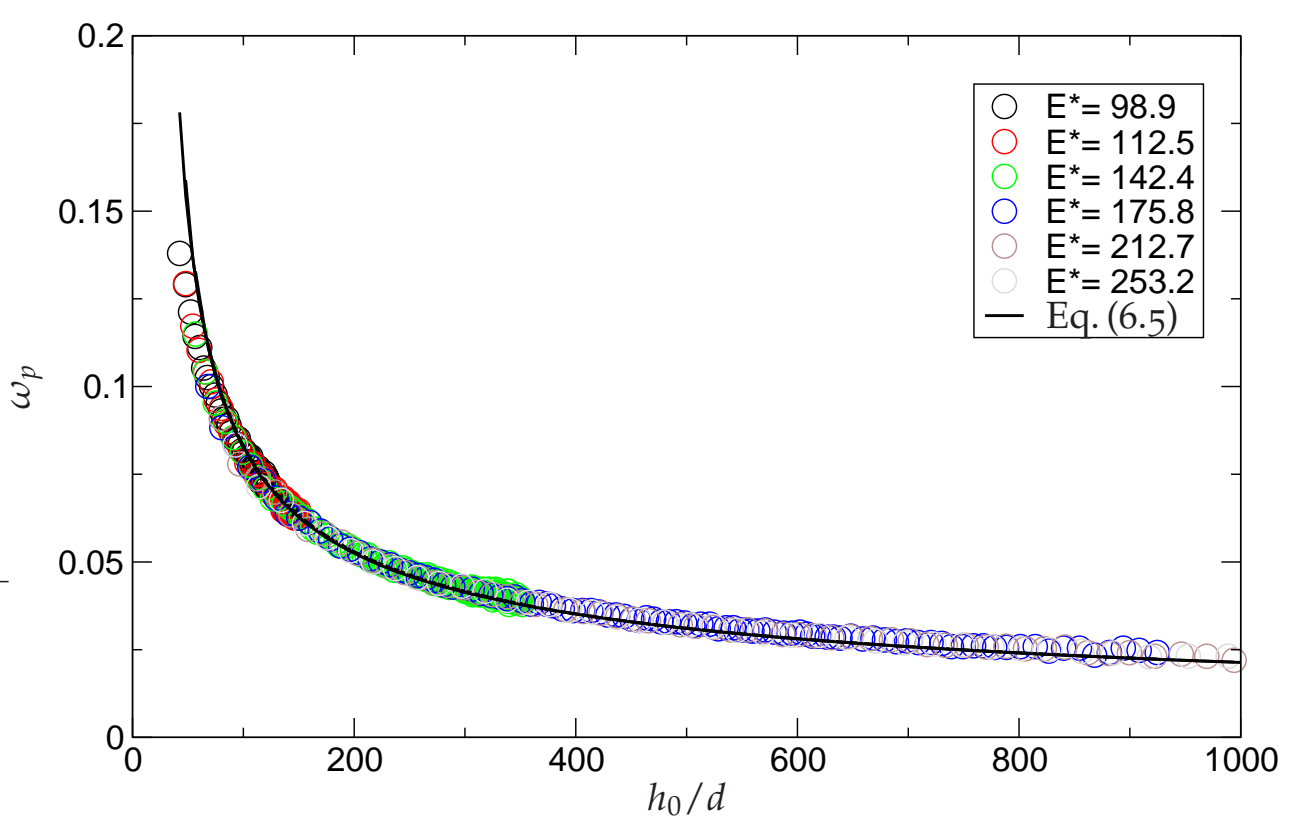

Fig. 6.11 Comparison of the oscillation frequency depending on the normalized mean height $h_{0} / d$ of simulations (circles) with the theoretical prediction (solid curve) yields good agreement. The simulations were performed in three dimensions with $3 \times 10^{4}$ particles, rupture distance of the capillary bridge $s_{\text {crit }} / d=0.0711$, driving acceleration $\Gamma=60.0$ and different driving energies $E^{*}$ as denoted in the legend.

the plug and the gas (b) grows and a gas bubble starts to be visible (c). This is most perceptible in the shadow of the snapshots which account for the areal density. In the last snapshot (d) the gas bubble increases and the cold plug is deformed into a "bubble and spike"-like shape, which is typical for Rayleigh-Taylor instabilities (Verdon et al., 1982; Sharp, 1983). We show a graph in (e), taken from Verdon et al. (1982), which shows the "bubble and spike" shape of the Rayleigh-Taylor instability in the flow of an ideal fluid layer. This underlines the similarity between the emerging structures in ideal fluids and wet granular matter.

To study the onset of the Rayleigh-Taylor instability in wet granular matter in more detail, the simulations are compared with the theoretical model. The onset of the Rayleigh-Taylor instability is described by Eq. (6.28), which is a convenient notation for comparing it with simulations, and reads in lowest order approximation

$$
L_{c}=2 \pi l
$$

where $L_{c}$ is the critical width of the plug and $l$ is the capillary length.

The capillary length, $l$, is not directly accessible for wet granular matter. We estimate it by using the equations which are valid for classical fluids, justified by the similarity of the emerging structures. The thickness, $h_{p}$, of a puddle of classical liquid is calculated in de Gennes et al. (2003) for simple fluids by balancing the surface tension $(2 \gamma)$ with the hydrostatic force of the puddle $\left(\rho g h_{p}^{2} / 2\right)$, both per unit 
length, and find

$$
h_{p}=2 l
$$

The height of the liquid puddle, or in the case of granular matter, the height of the plug, $h_{p}$, is accessible in the simulation and therefore provides an estimate for the capillary length.

We plug Eq. (6.32) into Eq. (6.31) and find the critical width of the plug, $L_{c}$, where the Rayleigh-Taylor instability occurs, to be

$$
L_{c}=\pi h_{p}
$$

In order to investigate this behavior in wet granular matter, we performed several simulations of a system with a square base area, where each edge is of length $L_{c}$. We keep the filling height constant at 48 Layers and therefore change the number of particles used (between $3 \cdot 10^{4}$ and $5.3 \times 10^{5}$ ). The variation of the parameter $h_{p}$ is done by approaching the stationary state of the system, through increasing the height of the vapor cushion $h_{0}$ which simultaneously reduces the height $h_{p}$ of the plug (cf. Fig.6.3b). As soon as bubbling is observed, we know the height $h_{p}$ for which $L_{c}$ is the critical width of the plug. The result of this analysis is displayed in Fig. 6.13. The solid red line in this graph is the theoretical prediction of Eq. (6.33). Looking at the original noisy data in Fig.6.13, this is in reasonable agreement.

\subsection{Summary and Outlook}

We find that the density inversion in wet granular matter bears striking qualitative similarities to the Leidenfrost effect of common liquids. Hence, we denote it as wet granular Leidenfrost effect. The reduction of heat conductivity does result in a pronounced interface between the gas cushion and the condensed plug hovering on it. The clear interface between the two phases is in contrast to dry granular matter.

The floating plug undergoes a smooth oscillation with a frequency not related to the external driving frequency. In fact, we find the oscillation frequency of the plug being well described by a theoretical model which yields the same oscillation frequency as in the case of a passive plug hovering on a real gas which takes excluded volume effects into account (cf. Eq. (6.5)) and shows good agreement (cf. Fig.6.11) with simulations. In first order approximation the plug oscillation frequency only depends on gravity and the mean height of the gas cushion.

Allowing for a lateral gas exchange in the model, we also predict a RayleighTaylor instability (cf. Fig. 6.9) to occur, which is reminiscent of bubbling in water (Biance et al., 2003). The predicted ratio of the length of the system divided by the critical height of the plug is in first order approximation $L_{c} / h_{p} \approx \pi$ above which the instability occurs. We find (cf. Fig.6.13) qualitative agreement of the simulations with the theoretical predictions. 
a

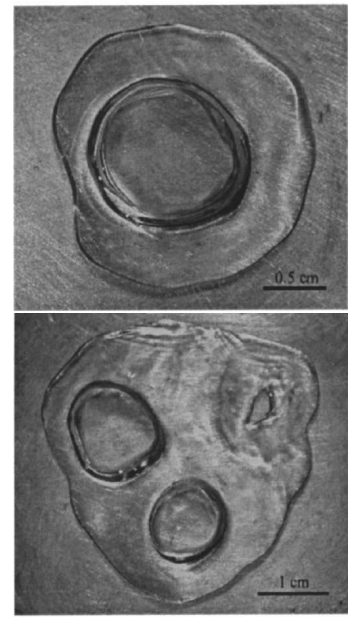

e
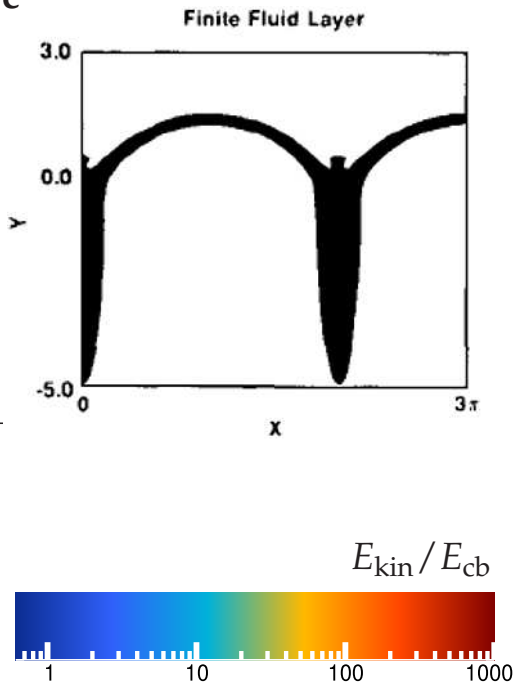
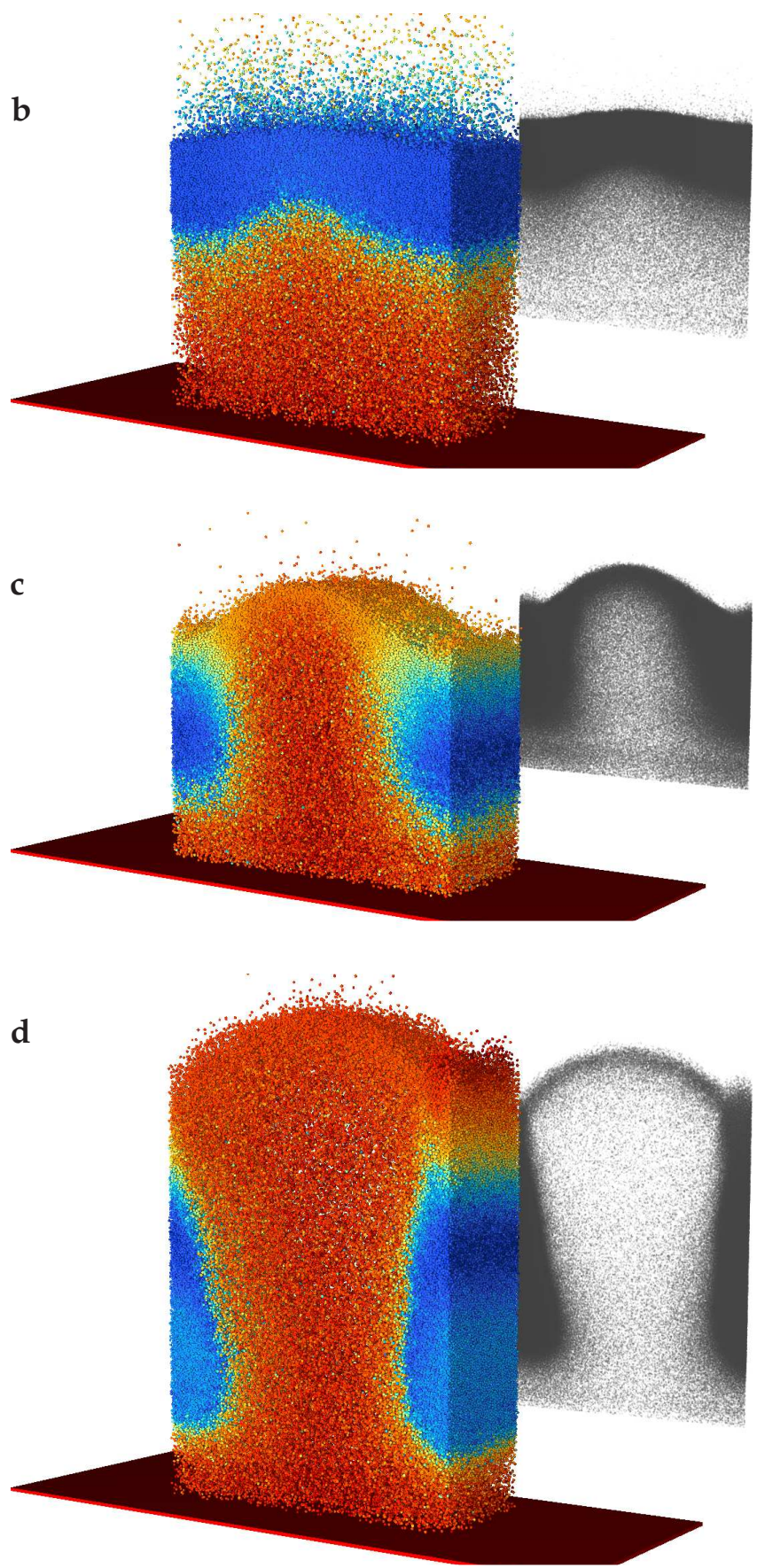

Fig. 6.12 a Top view of large water puddles placed onto a hot surface and obeying the Leidenfrost effect. Above a certain critical puddle size bubbles rise and burst at the surface. Depending on the size of the puddles (note the scale bars) even multiple bubbles can rise simultaneously. Image reprinted with permission from Biance et al. (2003). Copyright 2003, American Institute of Physics. b-d Successive Snapshots of a simulation of $3 \times 10^{5}$ wet granular particles show a rising gas bubble similar as in water. Note the shadow, which accounts for the areal density, where the bubble is most apparent. The colors represent the normalized kinetic energy per particle. The box is $125 d$ in width and $50 d$ deep. The driving parameters are: $\Gamma=60.0$ and $E^{*}=98.9$. e Plot of the interfaces of the Rayleigh-Taylor instability in layered flow of a finite ideal fluid layer as obtained by a two-dimensional numerical simulation. The "bubble and spike" structure in this ideal fluid simulation is reminiscent of the structure evolving in wet granular matter as can be seen in the shadow of snapshot $\mathbf{d}$ in this figure. The figure was reprinted from Verdon et al. (1982, Fig. 13). Copyright 1982, American Institute of Physics. 


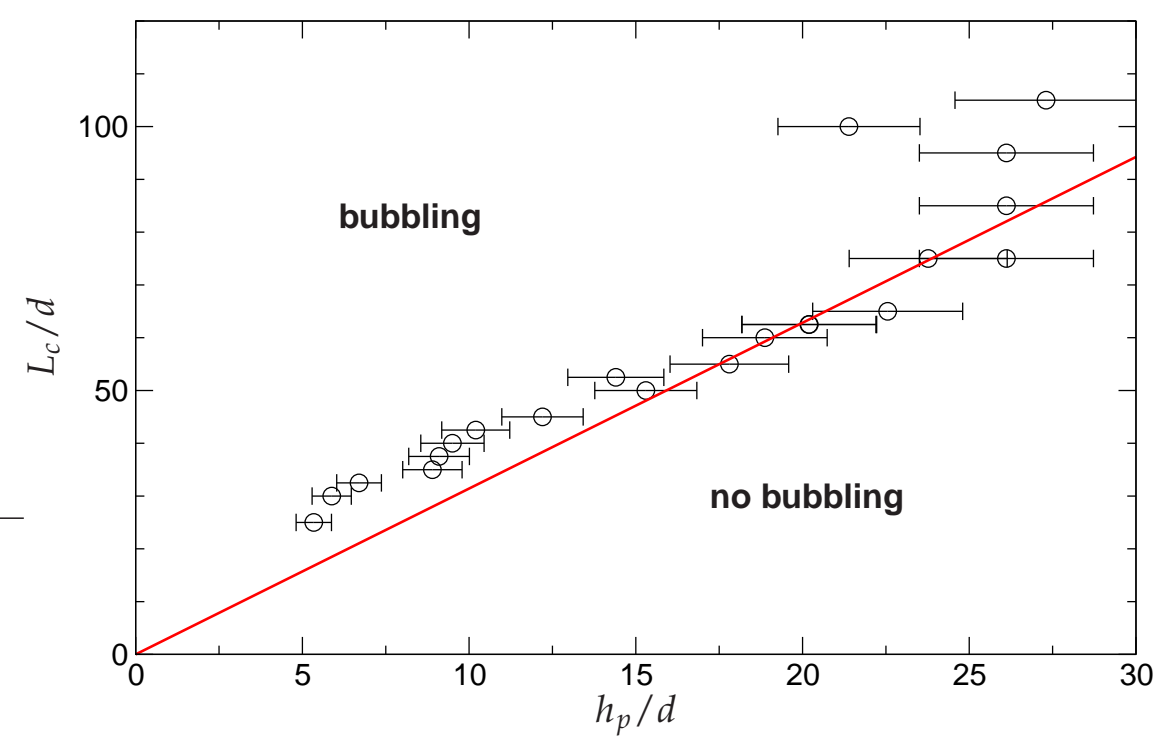

Fig. 6.13 The critical width of the plug, $L_{\mathcal{C}}$, is plotted against the critical height $h_{p}$ where the plug becomes unstable and shows the Rayleigh-Taylor instability. The circles are simulations of different amounts of wet particles contained by a square based box with side lengths $L_{\mathcal{C}}$. Different driving parameters are used in this plot. The solid red line is the theoretical prediction of Eq. (6.33).

\section{A. Inelasticity damps plug oscillation}

If we additionally introduce slightly inelastic collisions with a constant coefficient of restitution $\varepsilon=0.98$ between particles and $\varepsilon=0.99$ between particles and the wall, we can observe that the oscillation of the plug is damped (cf. Fig.6.14 top). Only for higher driving energies $E^{*}$ (cf. Fig.6.14 bottom), plug oscillations occur again. The amplitude of the oscillation is, in contrast to the elastic case, not constant but undergoes irregular fluctuations. The critical driving energy $E_{\text {onset }}^{*}$ necessary to obtain oscillations is found to be $\approx 2750$ which can be seen in Fig. 6.15. There, the root mean square of the plug excursion, $\varepsilon_{h}$, is plotted versus the driving energy $E^{*}$. To calculate this quantity, only the data in the stationary state of the simulation is used (which is for example $t / T_{\text {driv }}>267$ for the simulation in Fig.6.14 top). For driving energies smaller than $E_{\text {onset }}^{*}$ we obtain a non-zero value for $\varepsilon_{h_{\mathrm{rms}}}$ because of fluctuations in the data. Due to the comparatively large inelasticity of real systems, it is extremely difficult to observe these oscillations in real experiments. 

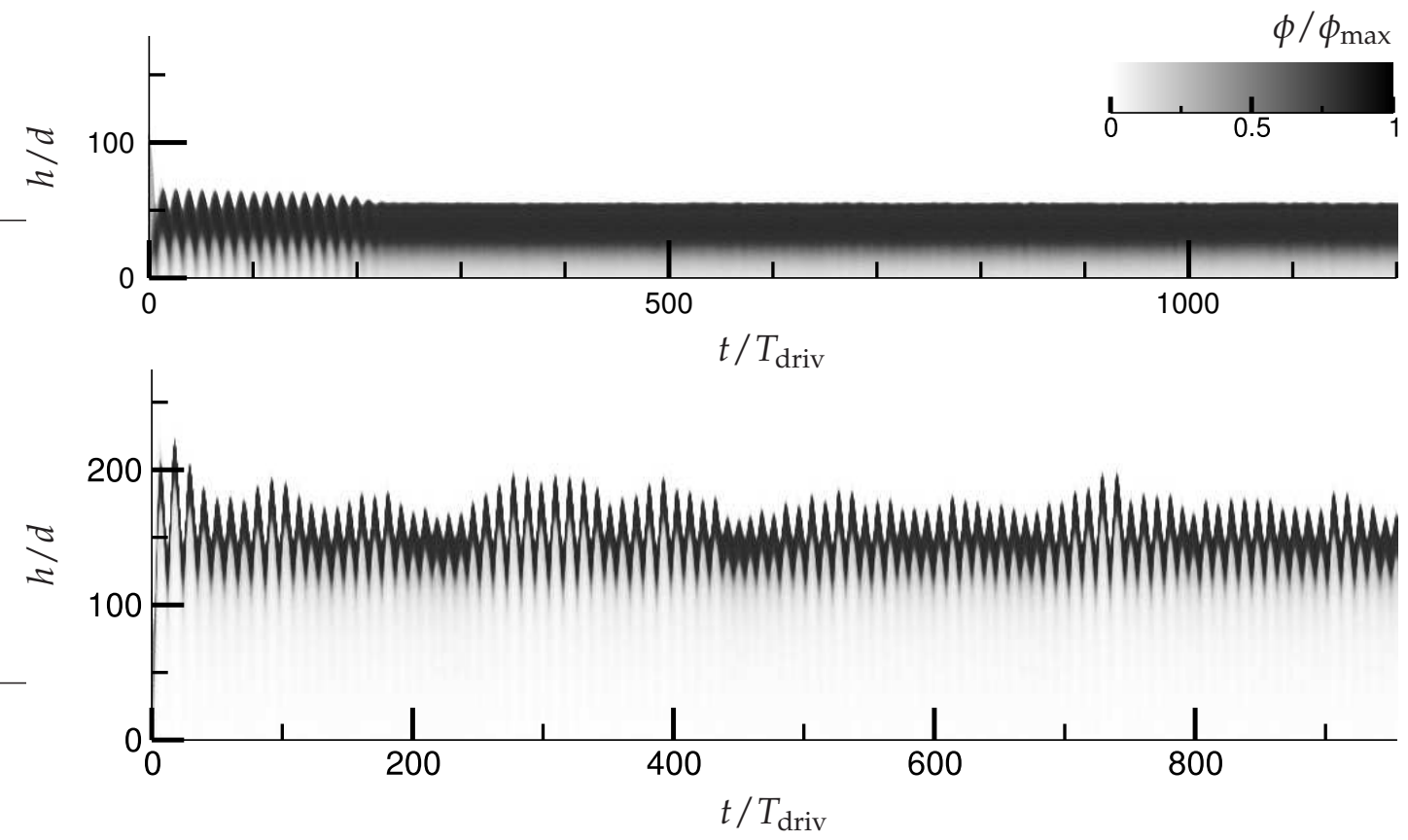

Fig. 6.14 Areal density (gray value) plotted as a function of height and normalized time $t / T_{\text {driv }}$. These simulations are performed for slightly inelastic particles with a coefficient of restitution of $\varepsilon=0.98$ between particles and $\varepsilon=0.99$ for collisions between particles and the wall. The maximum acceleration of the driving is $\Gamma=60.0$. The driving energies are $E^{*}=344.6$ (top) and $E^{*}=4395.4$ (bottom), respectively, while the rupture distance is $s_{\text {crit }} / d=0.0711$. top: The initially visible plug oscillation is damped and vanishes. bottom: An oscillation of the plug appears again for higher driving energies of the wall, however, it is not as stable as in the elastic case.

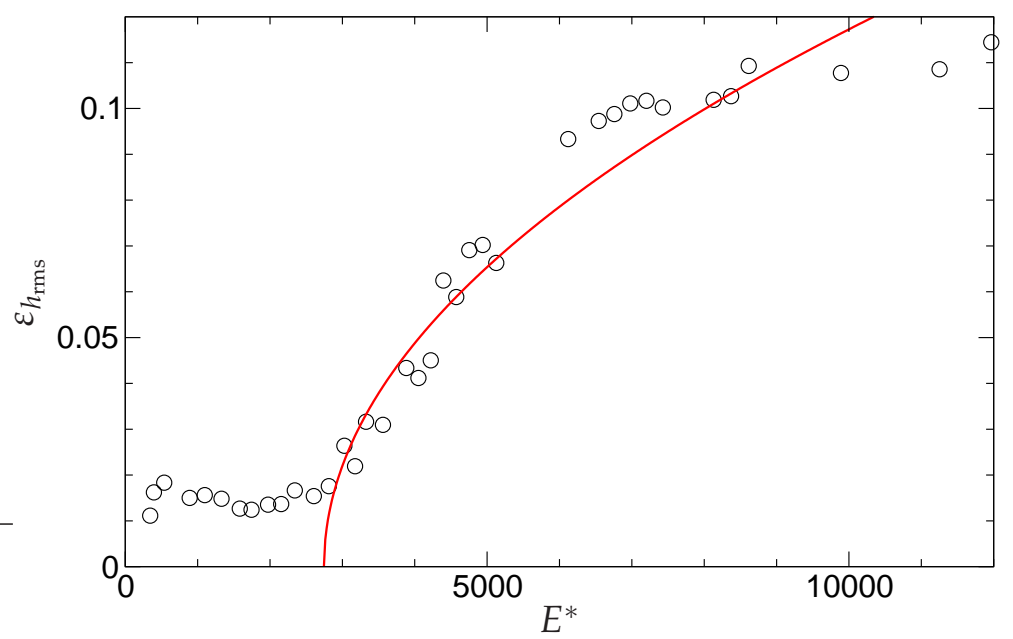

Fig. 6.15 Root mean square of the excursion $\varepsilon_{h}$ plotted versus the driving energy $E^{*}$. Circles denote values obtained by simulations and the red curve is a fit of the form $\varepsilon_{h_{\mathrm{rms}}} \propto\left(E^{*}-E_{\text {onset }}^{*}\right)^{1 / 2}$ with $E_{\text {onset }}^{*} \approx 2750$. The oscillation sets in at a critical driving energy $E_{\text {onset }}^{*}$ 


\section{Phase diagrams of wet granular matter - sinusoidal shearing}

In the previous chapters of this thesis, energy was injected into the system via a vertical sinusoidal motion of the system boundaries. The other main driving mechanism which will be used in this, and the following chapter, is sinusoidal shearing. Even though both driving mechanisms exert a sinusoidally varying force to the system, there are significant differences. For sinusoidal shaking the driving force is uniform within the system but changes sinusoidally with time, and requires the existence of a physical boundary. For example, a flat, elastically reflecting wall as used in this thesis. In contrast, for shearing the driving force is uniform in time but is changing sinusoidally with the spatial position within the system. Furthermore, the driving force is directly applied to the particles irrespective of the system boundaries.

The phase diagrams obtained for sinusoidal shearing are presented in this chapter. They are obtained by numerical simulations of a three-dimensional granular system. This is accompanied by a simple theoretical model which is capable of predicting the solid and the fluidized state. In chapter 8 the emerging dynamics of the fluidized state is studied more closely.

\subsection{Introduction}

Shear forces are omnipresent, as they can be found wherever two surfaces are in relative motion to each other and thus are present in many natural as well as industrial systems. For example, the lubricating oil film inside the cylinders of an internal combustion engine is (as any viscous liquid would be) subject to shear forces. The study of these shear forces reducing the friction and wear by lubrication forms an entire interdisciplinary scientific field called tribology (Urbakh et al., 2004, and references therein).

An interesting phenomenon observed in nature are landslides or mudslides. They are, due to their destructiveness, a very important phenomenon which is related to granular materials. They occur when additional shear forces, which cannot be compensated by internal friction anymore, act on the surface of a hill. This can possibly be caused by heavy rain, which is absorbed by the soil and results in a 
shear force due to a non-homogeneously distributed additional mass as a result of the slow drainage of the water. Shear forces within granular matter also commonly emerge in industrial processes where granular matter has to be handled. This is the case, for example, in the mixing process of granulates using multiple counterrotating agitators.

Shear forces can be modeled in various forms. Experimentally studying shear forces in granular systems can be done for instance by constructing two counterrotating walls (Liao and Hsiau, 2010, and references therein). In simulations, shear forces are applied to the system for example through a space-dependent external force field, $F(x)$, acting individually on each particle. In simulations it is often favorable to use periodic boundary conditions. This can be done by using a triangular shear profile, which is a combination of two linear shear profiles of the same slope but different sign. This linear shear profile would be non-differentiable at the system boundaries, so a cosine-shaped profile is used in this work. The shear force reads $F_{s}(x)=F_{\text {smax }} \cos \left(2 \pi x / L_{x}\right)$, where $L_{x}$ is the width of the system and is shown in Fig. 2.7 (Hoover, 1983; Schulz et al., 2003; Schulz and Schulz, 2006; Rahbari et al., 2009).

In the next section of this chapter a simple model is derived which captures the basic features of the transition from a solid to a fluidized state in sheared wet granular matter. Afterwards, this theoretical model is compared to three-dimensional event-driven simulations using the thin-thread model.

\subsection{Fluidization point of sheared wet granular matter}

In order to study the transition from a solid to a fluidized state in sheared wet granular matter, a simple model is proposed in the spirit of section 4.2. There a simple balance of forces acting in a one-dimensional column was readily able to predict the solid-fluid transition induced through vertical shaking. The system is reduced to its simplest geometry and only the force induced by the cosine-shaped shear field and the capillary bridge shall be considered.

The simplest wet granular system conceivable is a one-dimensional chain of particles - connected by capillary bridges. The chain spans through the system of length $L_{x}$ and the shear force is applied perpendicular to it. This is sketched in Fig.7.1. Here, the cosine-shaped shear force, $F_{S}(x)$, is shown at the top, which acts along the vertical direction, $y$ with a maximum force of $F_{\text {smax. }}$. In the lower part of the figure, a one dimensional chain of particles is displayed which shows a slight cosineshaped deformation with Amplitude $A_{l}$ in the vertical direction and resembles the cosine shape of the shear force. The mass density, $\rho_{m}$, is considered to be approximately the mass of one particle per particle diameter, which is $\rho_{m}=m / d$, assuming monodisperse particles of diameter $d$ and of equal weight $m$. The capillary bridges acting between the particles exert a force, $F_{B}$, which is assumed to be non-zero if the particles are in contact. This is the case for instance in the minimal capillary model. 


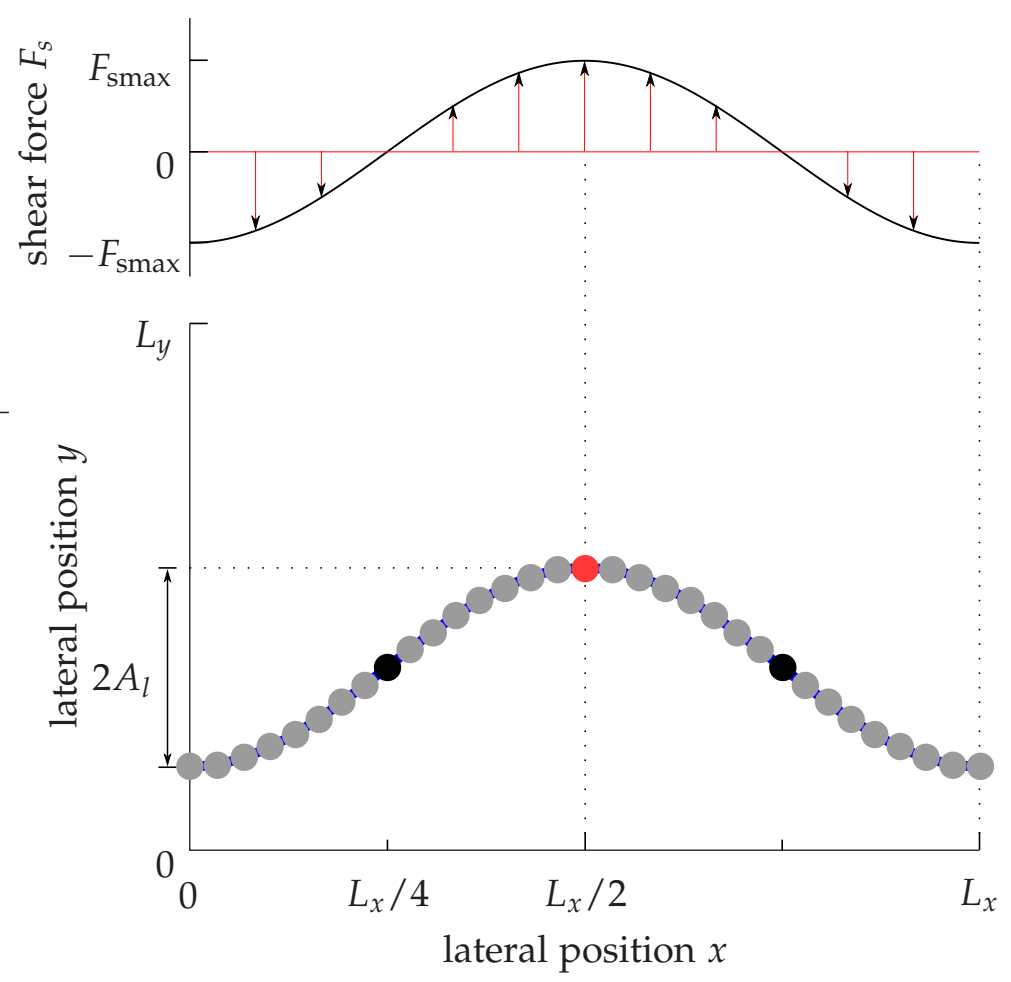

Fig. 7.1 This is a sketch of the theoretical simplification. The cosine-shaped shear force which varies in magnitude along the $x$ direction is indicated at the top with a maximum force $F_{\text {smax }}$. Below that, a one dimensional chain of particles is shown, which is deformed with amplitude $A_{l}$ and resembles a cosine shape. The particles are connected via capillary bridges which exert the capillary bridge force $F_{B}$ such that the particles are attracted and kept in contact. The strongest force in this chain acts on the black particles and therefore the capillary bridges connected to these particles are the first ones which will rupture. In contrast to that is the red particle which experiences the lowest force. The width of the system in the horizontal direction is $L_{x}$. 
The stability of the chain is associated with the state of the wet granular material. If the chain is stable and does not rupture under the applied shear force, this indicates a solid state. If the chain however ruptures, this indicates a fluid state. The chain is stable if the maximum force in the chain is smaller or equal to the capillary bridge force.

The shape of the chain has to be known in order to be able to calculate the maximum force within the chain. The calculation of the shape is reminiscent of the calculation of the curve of a free-hanging chain in a uniform gravitational field. There the resulting shape is called catenary and its derivation is a standard textbook example for variational calculus. The main difference between the catenary situation and the present system is the non-uniformity of the acceleration in the present system, which is caused by the spatially varying shear force. The analogy to the catenary also directly indicates that the forces in the present model are not distributed uniformly within the chain. The strongest forces act on the black particles in Fig.7.1 which are located at $x=L_{x} / 4$ and $x=3 L_{x} / 4$. This immediately shows that the capillary bridges connected to the black particles are the first ones which will rupture if the shear force becomes too large. Nevertheless, the solution is obtained by using the Euler-Lagrange equation to minimize the Lagrangian. A Lagrange multiplier, $\lambda_{l}$, is used to take the constraint into account that the length of the chain shall be constant. The following calculation assumes vanishing granular temperature, $T_{g} \rightarrow 0$, such that there is no kinetic but only potential energy within the chain. The potential energy of the chain reads

$$
E_{\mathrm{pot}}=F_{\mathrm{smax}} \frac{L_{x}^{2}}{d} \int_{-1 / 4}^{1 / 4} \cos (2 \pi \tilde{x}) \tilde{y} \sqrt{1+\tilde{y}^{\prime 2}} \mathrm{~d} \tilde{x}
$$

where $\tilde{x}=x / L_{x}$ is the normalized position in the horizontal direction, $\tilde{y}(\tilde{x})=$ $y(x) / L_{x}$ is the position of the chain in the vertical direction and $\tilde{y}^{\prime}(\tilde{x})=y^{\prime}$ is its normalized derivative with respect to $\tilde{x}$. The derivative does not change as both spatial coordinates are normalized by the same length $L_{x}$. The length of the chain is

$$
l_{\text {chain }}=L_{x} \int_{-1 / 4}^{1 / 4} \sqrt{1+\tilde{y}^{\prime 2}} \mathrm{~d} \tilde{x}
$$

Therefore, the variational derivative to minimize the Lagrangian reads

$$
\delta\left(\tilde{E}_{\text {pot }}+\tilde{\lambda}_{l} \tilde{l}_{\text {chain }}\right)=\delta\left(\int_{-1 / 4}^{1 / 4}\left(\cos (2 \pi \tilde{x}) \tilde{y}+\tilde{\lambda}_{l}\right) \sqrt{1+\tilde{y}^{\prime 2}} \mathrm{~d} \tilde{x}\right)=0,
$$

where $\delta$ is the variational derivative, $\tilde{E}_{\text {pot }}=E_{\text {pot }} d / L_{x}^{2} F_{\text {smax }}$ the normalized potential energy, $\tilde{\lambda}_{l}=\lambda_{l} d / L_{x} F_{\text {smax }}$ the normalized Lagrange multiplier and $\tilde{l}_{\text {chain }}=l_{\text {chain }} / L_{x}$ 
the normalized length of the chain. This is solved by the Euler-Lagrange equation

$$
\frac{\mathrm{d}}{\mathrm{d} \tilde{x}} \frac{\partial G}{\partial \tilde{y}^{\prime}}-\frac{\partial G}{\partial \tilde{y}}=0
$$

with

$$
G\left(\tilde{y}(\tilde{x}), \tilde{y}^{\prime}(\tilde{x}), \tilde{x}\right)=\left(\tilde{y} \cos (2 \pi \tilde{x})+\tilde{\lambda}_{l}\right) \sqrt{1+\tilde{y}^{\prime 2}} .
$$

The Euler-Lagrange equation is solved numerically and the resulting shape of the chain for $\tilde{\lambda}=0.4$ is shown in Fig.7.2 (black curve). Additionally, the function $\tilde{y}=-\tilde{A}_{l} \cos (2 \pi \tilde{x})$, with the normalized amplitude $\tilde{A}_{l}=A_{l} / L_{x}=0.07384$ is plotted in Fig. 7.2 (red curve). The amplitude of the cosine-shaped curve was chosen such that both curves have the same maximum value (at $\tilde{x}=0)$. It can be seen that the slightly thicker black curve is almost indistinguishable from the thinner red one. The deviations of the cosine approximation from the numerically solved full problem is shown as the relative error in the inset of the graph. It can be observed that the cosine approximation never deviates more than 3\% from the cosine-shaped function, which indicates a reasonable agreement. Thus, the shape of the chain is approximated in the following simply by a cosine-shaped function with an appropriate amplitude ${ }^{1}$.

Assuming the shape of the chain to be $\tilde{y}=-\tilde{A}_{l} \cos (2 \pi \tilde{x})$ leads to the following solutions for the potential energy and the length of the chain

$$
\begin{aligned}
\tilde{E}_{\text {pot }}\left(\tilde{A}_{l}\right) & =\left(a^{2}-1\right) \operatorname{Ei}_{E}\left(-a^{2}\right)+\left(a^{2}-1\right) \operatorname{Ei}_{E}\left(\frac{a^{2}}{1+a^{2}}\right) \sqrt{1+a^{2}} \\
& +\left(a^{2}+1\right) \operatorname{Ei}_{K}\left(-a^{2}\right)+\operatorname{Ei}_{K}\left(\frac{a^{2}}{1+a^{2}}\right) \sqrt{1+a^{2}} \\
\tilde{l}_{\text {chain }}\left(\tilde{A}_{l}\right) & =\frac{1}{\pi} \operatorname{Ei}_{E}\left(-a^{2}\right)
\end{aligned}
$$

with the abbreviation $a=2 \pi \tilde{A}_{l}$ and where $\mathrm{Ei}_{K}$ and $\mathrm{Ei}_{E}$ is the complete elliptic integral of the first and second kind, respectively. The solutions only depend on the amplitude $\tilde{A}_{l}$. The maximum force acting in the chain, $F_{\text {chain, }}$ can now be calculated by

$$
\tilde{F}_{\text {chain }}=-\frac{\mathrm{d} \tilde{E}_{\text {pot }}}{\mathrm{d} \tilde{A}_{l}}\left(\frac{\mathrm{d} \tilde{l}_{\text {chain }}}{\mathrm{d} \tilde{A}_{l}}\right)^{-1}
$$

where $\tilde{F}_{\text {chain }}=F_{\text {chain }} d / F_{\text {smax }} L_{x}$ is the normalized force within the chain. The maximum force in the chain can be evaluated using numerical values for the elliptic integrals and is plotted in Fig. $7 \cdot 3$ as a function of the amplitude $\tilde{A}_{l}$ of the chain. The result seems reasonable as it recovers the correct limits. For $A_{l} \rightarrow 0$ the force in the chain goes to infinity, $\tilde{F}_{\text {chain }} \rightarrow \infty$, because the tension in the chain becomes infinite.

1. This assumption was already used in Sec. $5 \cdot 3$ for the calculation of the interfacial energy and hereby is additionally justified. 


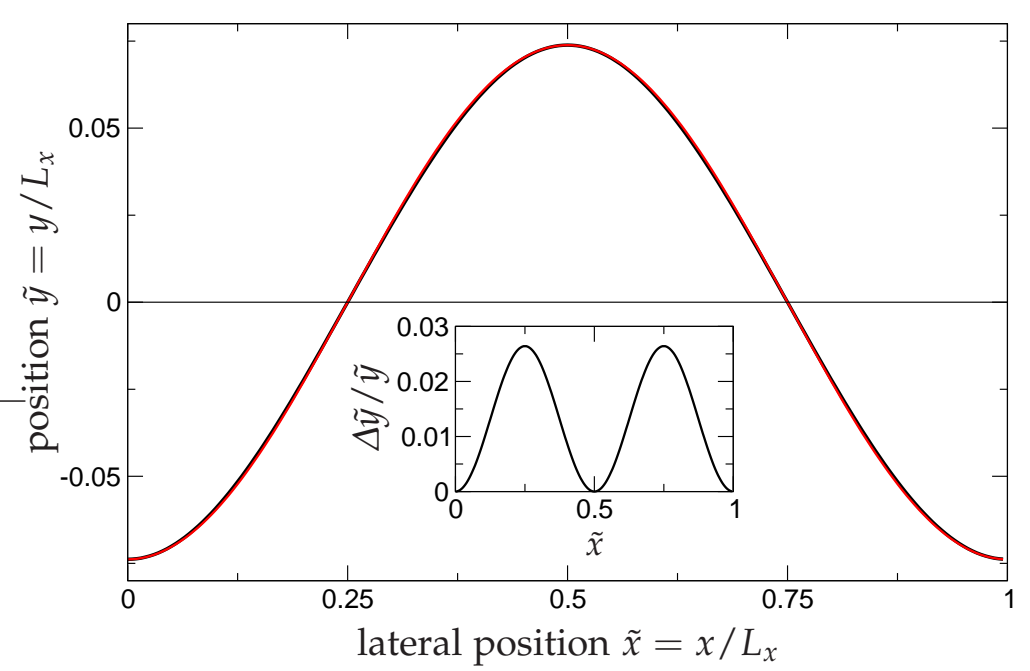

Fig. 7.2 The thicker black line is a numerically obtained solution of the Euler-Lagrange equation Eq. (7.4) of the full problem and gives the resulting shape of the chain of particles in a continuum approximation for $\tilde{\lambda}=0.4$. The thinner red line is the curve $\tilde{y}=-\tilde{A}_{l} \cos (2 \pi \tilde{x})$ with $\tilde{A}_{l}=A_{l} / L_{x}=0.07384$. It can be observed that the two curves are almost indistinguishable and therefore in the inset the relative error, $\Delta \tilde{y} / \tilde{y}$, of the cosine function is shown. It can be seen that there is indeed a deviation which, however, is well below three percent. This justifies that a cosine approximation of the shape of the chain can be used. The amplitude of the cosine function was chosen such that the two curves match at $\tilde{x}=0$ and it can be seen that the numerical solution goes a bit straighter from one extremum to the other than the cosine.

For $A_{l} \rightarrow \infty$ the force in the chain also goes to infinity, $\tilde{F}_{\text {chain }} \rightarrow \infty$, because the length and therefore the mass of the chain becomes infinite. In between, there is an optimal amplitude, $\tilde{A}_{\text {opt }}=0.1912$, which leads to the lowest force $\tilde{F}_{\text {cmin }}=0.2709$ (dashed lines in Fig.7.3) within the chain and therefore to the highest stability against the applied shear force. The length of this chain, which spans the whole system, is $\tilde{l}_{\text {chain }}=1.2970$. If $\tilde{F}_{\text {chain }}<\tilde{F}_{\text {cmin }}$ which is indicated as the blue shaded area in Fig. $7 \cdot 3$, then there is no solution and thus no stable configuration of the chain which can withstand the applied shear force. This is the fluidized state. In contrast, if $\tilde{F}_{\text {chain }}>\tilde{F}_{\text {cmin }}$, there is a comparatively large range of amplitudes, which lead to stable chains in the system and thus lead to a solid state (gray shaded area). The different stable chain lengths are shown in Fig.7.3, where, assuming a certain fixed value for $\tilde{F}_{\text {chain }}$ (for example $\tilde{F}_{\text {chain }}=0.5$, dotted line), all chains are stable whose amplitudes, $\tilde{A}_{l}$, lie within the range denoted by the arrow. This is particularly interesting if assessing the transferability of this theoretical approach to real granular systems in two or three spatial dimensions. Due to the rearrangement of particles and capillary bridges within sheared granular matter, the force chains which stabilize the system undergo certain changes and fluctuations. However, due to the large range of possible geometries of the force chains which lead to stable configurations, this helps to stabilize the system against small fluctuations. Therefore, if the model is realistic it should be possible to find a wide distribution of force chain amplitudes 


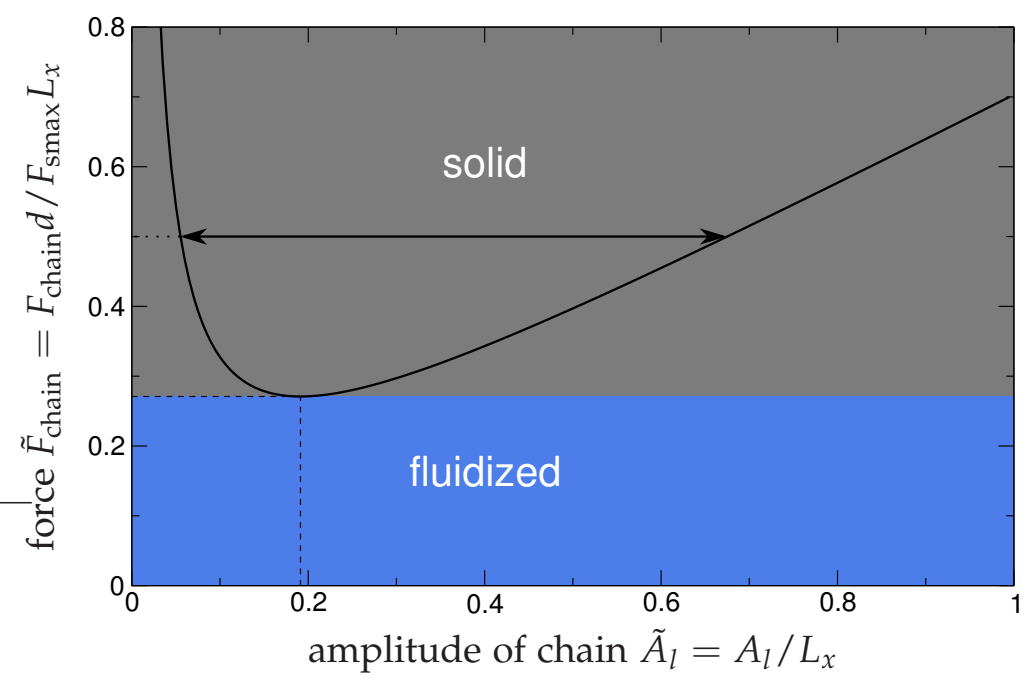

Fig. 7.3 The curve shows the normalized force $\tilde{F}_{\text {chain }}=F_{\text {chain }} d / F_{\text {smax }} L_{x}$, which acts on the black particles in Fig.7.1 and is shown as a function of the normalized amplitude of the chain, $\tilde{A}_{l}=A_{l} / L_{x}$ as obtained by Eq. (7.8). The two limits $\tilde{A}_{l} \rightarrow 0$ and $\tilde{A}_{l} \rightarrow \infty$ correctly lead to an infinite force, which is caused by an infinite tension in the chain in the first case and an infinite mass of the chain in the second case. An optimum chain amplitude, $\tilde{A}_{\text {opt }}=0.1912$, where the force is minimal, $\tilde{F}_{\text {cmin }}=0.2709$, is obtained (dashed lines). The absence of a solution for $\tilde{F}_{\text {chain }}<\tilde{F}_{\text {cmin }}$ indicates the fluidized state (blue shaded region), where the chain cannot find a stable configuration. Accordingly, the solid state is obtained for $\tilde{F}_{\text {chain }}>\tilde{F}_{\text {cmin }}$ (gray shaded region). In this regime, assuming a fixed value for $\tilde{F}_{\text {chain }}$ (for example $\tilde{F}_{\text {chain }}=0.5$, dotted line), all amplitudes in the range denoted by the arrow lead to a stable configuration.

when inspecting granular materials in two or three dimensions.

The maximal force which the chain can withstand is given by the capillary bridge force and reads $F_{\text {chain }} \leqslant F_{B}$. A solid state is obtained if $\tilde{F}_{\text {chain }}<\tilde{F}_{\text {cmin }}$, where $\tilde{F}_{\text {cmin }}$ is the minimal force which is obtained at the optimal chain amplitude. This leads to the final result

$$
\frac{F_{\mathrm{smax}}}{F_{B}} \frac{L_{x}}{d} \leqslant \frac{1}{\tilde{F}_{\mathrm{cmin}}} \approx 0.9229,
$$

which is the criterion to obtain a stable chain and thus a solid state. The model assumes vanishing granular temperature and only is valid in this limit. Thus it is intrinsically independent of the direction of the transition between the two phases (solidification of fluidization).

\subsection{Phase diagrams}

The theoretical estimate as given by Eq. (7.9) for the transition from the solid to the fluidized state shall now be compared to three-dimensional simulations of wet granular matter using the thin-thread model. To this end, a flat system is used 
as shown in Fig. 2.7, which contains elastically colliding, spherical particles. It is known that polydisperse beads which are subject to small shearing forces show a visible segregation according to their size (Schulz et al., 2003). Hence, monodisperse spheres of diameter $d=1$ are used in this and the next chapter in order to suppress any additional dynamics arising from different particle sizes. The particles are also of the same mass $m=1$. Gravity is acting perpendicular to the shear force with an acceleration due to gravity of $g=1$. This is not crucial for the appearance of solid-fluid phase transitions in wet granular matter as they are present also in twodimensional systems without gravity (Schulz et al., 2003; Rahbari, 2009), but it is favorable as it creates a more or less well defined packing in the solid state. Since all particles accumulate at the bottom it can be assumed that the packing density initially before shearing is approximately random close packing with $\phi_{\text {rcp }} \approx 0.64$. The filling height is on average $h_{f} / d=5.0$ in the solid state and, since the upper wall is at height $7.5 d$, the packing fraction can go down at the transition from the solid to the fluidized state. Jamming is prevented because the system can extend in the vertical direction where it is only bounded by gravity (see, for example, Valverde et al., 2004, and references therein).

The theoretical picture of a rupturing chain led to the first result that the capillary bridges of the particles, which are located for example at position $L_{x} / 4$ (black particles in Fig.7.1), are the first ones to rupture if the applied shear force is slightly above the critical value for stability. A snapshot of a simulation which leads to a fluidized state is shown in Fig. 7.4 and shows the initial stage directly after formation of a shear band. In agreement with the theoretical picture, the shear band forms such that the capillary bridges in the region around $L_{x} / 4$ and $3 L_{x} / 4$ are ruptured, while in the other parts the network of capillary bridges remains intact. This supports the basic idea behind the theoretical estimate.

To take the comparison further, the phase diagrams obtained by numerical simulations are presented and the transitions are compared to the theoretical predictions by Eq. (7.9). Individual phase diagrams are presented which arise from the variation of each of the following control parameters. The critical rupture separation, $s_{\text {crit }}$, the width of the system, $L_{x}$, the capillary bridge force, $F_{B}$ and the filling height of particles in the system, $h_{f}$. The latter is estimated as the filling height in the solid state assuming random close packing $h_{f}=H \phi / \phi_{\text {rcp }}$, where $\phi$ is the average particle density in the system. The theoretical prediction, which is given by Eq. (7.9), contains only the parameters $L_{x}$ and $F_{B}$ and thus is independent of $s_{\text {crit }}$ and $L_{x}$. Thus the model is valid in the limit $s_{\text {crit }} \rightarrow 0$ and at $h_{f}=1$.

Before discussing the results the exact method of how the parameters are varied is explained. The variation of the critical rupture separation, $s_{\text {crit }}$, was done by changing the capillary bridge energy, $E_{\mathrm{cb}}$, while the capillary bridge force was kept constant. The variation of the system width, $L_{x}$, was done whilst keeping the aspect ratio of the container constant at $L_{y} / L_{x}=2$. At the same time the average particle number density was kept constant at $\phi=0.43$ which means that the number of particles is changing. The variation of the capillary bridge force, $F_{B}$, was done by 


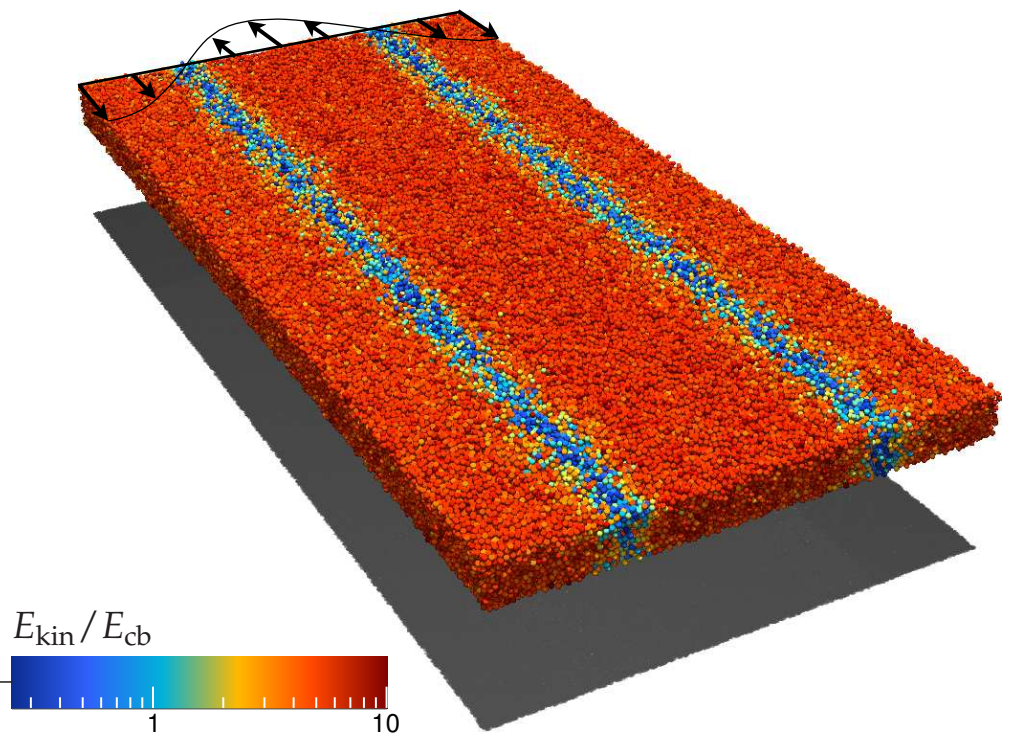

Fig. 7.4 A snapshot of a three-dimensional simulation of $2.15 \times 10^{5}$ monodisperse, elastically colliding wet granular spheres is shown, which is simulated using the thin-thread model. The flow is driven by a cosine-shaped shear force field $F_{S}(x)$, which is sketched on the figure. Similarly, as predicted by the theoretical model, the capillary bridges in the region around $L_{x} / 4$ and $3 L_{x} / 4$ are the first ones which rupture, whereas the other capillary bridges are still stable. This gives rise to the shear bands which are observed in this snapshot. The capillary interaction is characterized by $s_{\text {crit }} / d=0.0625$ and $E_{\mathrm{cb}} / m g s_{\mathrm{crit}}=6.0$, with a filling height of $h_{f} / d=8.8$. The maximum shear force is $F_{\mathrm{smax}} L_{x} / F_{B} d=$ 2.5 such that $F_{\text {smax }} / F_{\text {scrit }}=1.11$, where $F_{\text {scrit }}$ is the critical shear force for the transition to occur. The color of each particle indicates its individual kinetic energy and the system is $L_{x}=100 \mathrm{~d}$ in width, $L_{y}=$ $200 d$ in length and $H=12.5 d$ in height. The system starts in a fluidized state with homogeneously distributed particles and a gaussian velocity distribution of mean granular temperature $T_{g} / E_{\mathrm{cb}}=40.0$.

changing the capillary bridge energy, $E_{\mathrm{cb}}$, while keeping $s_{\text {crit }}$ constant. The filling height, $h_{f}$, was varied by changing the number of particles in the system while keeping the geometry of the simulation volume fixed.

All parameters which were constant during any of these simulations took the values as following. The number of monodisperse particles with diameter $d$ in the system was $4.39 \times 10^{4}$. Each particle was of mass $m$ and collisions were elastic. The particles experienced an acceleration due to gravity of $g$. The capillary interaction of the thin-thread model was given by $E_{\mathrm{cb}} / m g s_{\text {crit }}=6.0, s_{\text {crit }} / d=0.0625$ and $F_{B} / m g=$ 6.0. The system was of size $L_{x} / d=60, L_{y} / d=120$ with a height $H / d=7.5$, which lead to a filling height of $h_{f} / d=5.0$. The particles are initially homogeneously distributed within the system with a Gaussian velocity distribution of mean granular temperature $T_{g} / E_{\mathrm{cb}}=40.0$.

The resulting phase diagrams are shown in Fig. $7 \cdot 5$. The phase transition was detected by the order parameter which is defined as the mean kinetic energy parallel to the driving, $E_{\|}$. As briefly introduced in Sec.2.4.4, a significant non-zero value indicates a fluidized state. The phase boundary is shown as red circles and calculated 
as mean value between the neighboring values, which belong to different states. The red lines are fits to the phase boundary using an empirical fit function which best fits the data with the result being noted below each phase diagram. The white dashed lines in Fig. 7.5(a)-(c) indicate the transitions as predicted by the theoretical model in Eq. (7.9). Now the phase diagrams are discussed individually.

The phase diagram for the variation of the critical rupture separation, $s_{\text {crit }}$, is shown in Fig.7·5(a). The linear fit to the phase boundary was chosen such that it has the same intercept with the vertical axis as predicted by the model. The values obtained for the phase boundary out of the simulations nicely fall on this curve. This suggests that the force chain model is in good agreement with the simulations in the limit $s_{\text {crit }} \rightarrow 0$. However, as all of the following simulations in the other phase diagrams are performed at a critical rupture separation of $s_{\text {crit }} / d=0.0625$, no quantiative agreement can be expected any longer. In the following, a simple argument indicates the mechanism by which the fluidization threshold becomes a function of the critical rupture separation. A larger value of $s_{\text {crit }}$ enables each particle to maintain more capillary bridges to neighboring particles. This was shown in a simulation of freely cooling, two-dimensional granular matter in (Fingerle and Herminghaus, 2008, Fig. 9). At the same time an increase in the number of capillary bridges per particle leads to an increases in the total binding energy per particle. If the particle shall be driven into the fluidized state, more energy is necessary to rupture all liquid bridges and thus more energy has to be injected by the driving. This can, for example, be done with an increase of the maximum driving shear force and thus suggests the increase in the fluidization threshold as observed in the experiments. As the forces involved in the increase of the binding energy are not aligned along a specific direction this is a purely energetic argument. Therefore, the stability of the force chain seems not to be increased in an obvious manner. Ergo this dependency of the fluidization threshold on the critical rupture separation cannot be included into the force chain model, for example, simply via a larger effective capillary bridge force.

The phase diagram for the variation of the width of the system, $L_{x}$, is shown in Fig. $7 \cdot 5(\mathrm{~b})$ and shows very good qualitative agreement with the theoretical prediction: it is constant. This is valid in the whole range of system sizes studied here. Both, the simulation and the theory state that in the limit of very large system sizes, $L_{x} \rightarrow \infty$, a fluidized state is found for any finite shear force, $F_{\text {smax }}$. This is reasonable as the mass of the chain then becomes infinite and thus cannot be balanced by a finite capillary bridge force anymore. It was confirmed in simulations (not presented here) that the value of $L_{y}$, with $L_{x}$ kept fixed, did not have a significant influence on the fluidization transition.

The phase diagram obtained by the variation of the capillary bridge force, $F_{B}$, is shown in Fig. $7 \cdot 5(\mathrm{c})$ and shows reasonable qualitative agreement with the theoretical prediction for large values of $F_{B}$. However, a qualitative different behavior is found in the limit of vanishing capillary bridge forces. A short argument shall be suggested why this deviation seems reasonable. To this end the resulting 


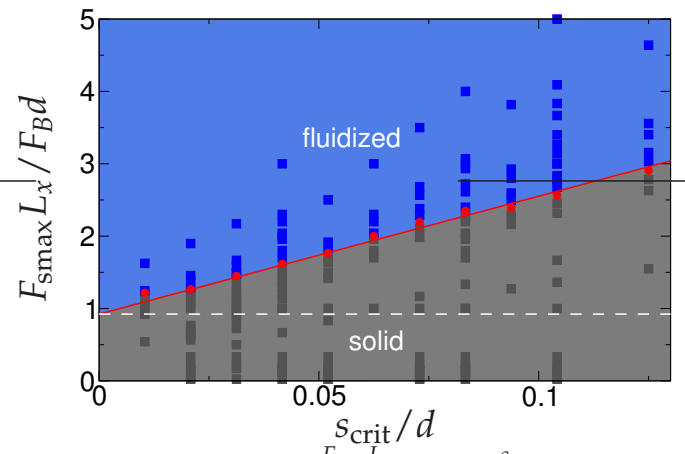

(a) Phase boundary: $\frac{F_{\text {scrit }} L_{x}}{F_{B} d}=16.3 \frac{s_{\text {crit }}}{d}+0.9229$.

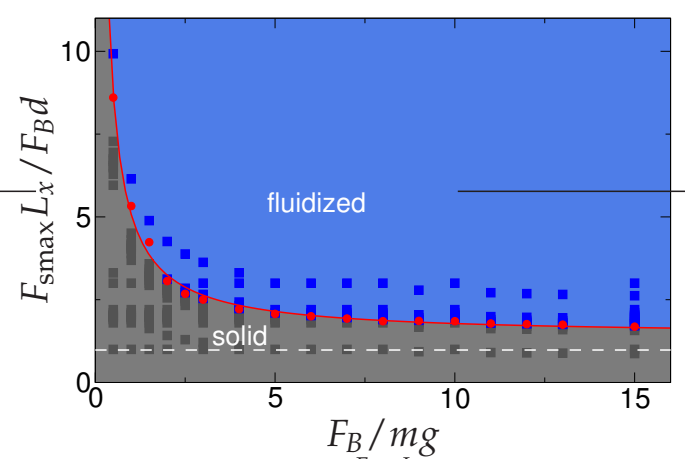

(c) Phase boundary: $\frac{F_{\text {scrit }} L_{x}}{F_{B} d}=3.7 \frac{m g}{F_{B}}+1.4$.

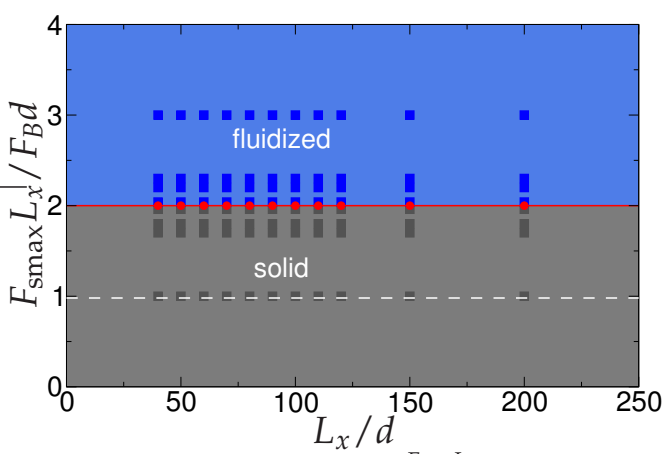

(b) Phase boundary: $\frac{F_{\text {scrit }} L_{x}}{F_{B} d}=2.0$.

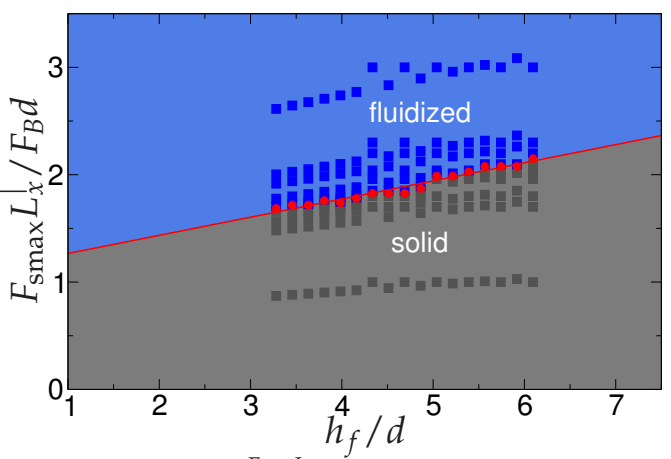

(d) Boundary: $\frac{F_{\text {scrit }} L_{x}}{F_{B} d}=0.17\left(h_{f} / d-1\right)+1.3$

Fig. 7.5 Phase diagrams which show the phase boundary between the solid and the fluidized state for different parameters. (a) critical rupture separation, $s_{\text {crit }}$, (b) system width, $L_{x}$, (c) capillary bridge force, $F_{B}$, and (d) the filling height $h_{f}$ estimated as the filling height in a solid state assuming random close packing $\phi_{\mathrm{rcp}} \approx 0.64$. Squares represent event-driven simulations using the thin-thread model which showed either solid (black) or fluidized (blue) behavior. Red circles show the (normalized) critical amplitude of the driving force, $F_{\text {scrit }}$, for which the transition was observed. The red line is obtained as a fit with the result stated below each diagram. The white dashed lines in subfigure (a)-(c) denote the results obtained from the theoretical model. Good agreement between simulations and theoretical estimate is obtained for the first two phase diagrams shown in (a) and (b). A more detailed discussion, the exact system parameters and the method for performing the simulations are given in the text. 
equation of the transition, as obtained by the fit, is written in the following form $F_{\text {scrit }} L_{x} / d=3.7 m g+1.4 F_{B}$. This states that in the limit $F_{B} \rightarrow 0$ still a finite shear force is necessary to fluidize the particles. This seems to depend on the gravitational force $F_{g}=m g$ acting on the particles. The transition from the solid to the fluidized state certainly is associated with a decrease in local density. This results in a dilation of the granular particles in the vertical direction. Thus the filling height of the system increases by a small amount $\Delta h_{f}$, which shall be estimated as follows. The potential energy which is necessary to increase the filling height from $h_{f}$ to $h_{f}+\Delta h_{f}$ is given by $E_{\text {pot }}=h_{f} m g \Delta h_{f} / 2$. In the limit $F_{B} \rightarrow 0$ it now is assumed that fluidization occurs if the driving provides enough energy for the dilation to occur. The energy $E_{\text {inj }}$ injected through the driving shear force for a small elongation $\varepsilon_{l}=\varepsilon_{0} \cos \tilde{x}$ is given by $L_{x} F_{\text {scrit }} \varepsilon_{0} / 2$. It is difficult to suggest a reasonable estimation for the distance $\varepsilon_{0}$. The assumption that fluidization occurs if the driving provides enough energy for the dilation to occur states that $E_{\text {inj }}=E_{\text {pot }}$ and leads to $F_{\text {scrit }} L_{x} / d=h_{f} \Delta h_{f} m g / d \varepsilon_{0}$. The quotient $\Delta h_{f} / \varepsilon_{0}$ is difficult to estimate ${ }^{2}$. By assuming it to be independent of $h_{f}$ at least the following qualitative scaling should be obtained $F_{\text {scrit }} L_{x} / d \propto h_{f} / d$. This scaling relation shall be examined in the last phase diagram. As this argument again is an energetic criterion it cannot be incorporated into the force chain model in an obvious manner.

Finally, the phase diagram for the variation of the filling height, $h_{f}$, is shown in Fig. $7 \cdot 5(\mathrm{~d})$ in order to support the assumption made above that a certain gravitational energy barrier has to be overcome for fluidization to occur. This leads to the expectation that in the limit $F_{B} \rightarrow 0$ the critical fluidization scales as $F_{\text {scrit }} L_{x} / d \propto h_{f} / d$. This means that the energy barrier created by the potential energy linearly increases with the number of particle layers. By linear regression of the phase boundary obtained in the present phase diagram to the limit $F_{B} \rightarrow 0$ the scaling indeed is found in the simulations. This supports the assumption that an potential energy barrier has to be overcome in order to fluidize the wet granular matter in the limit of vanishing capillary bridge forces.

\subsection{Summary and Outlook}

A simple theoretical model was developed in this chapter to describe the transition from a solid to a fluidized state in sheared wet granular matter. The model consists of a single one-dimensional chain of particles where the particles are connected through capillary bridges. The chain is spanned through a system of width $L_{x}$ perpendicular to the applied shear force. The theoretical model, naturally connects the stability of the one-dimensional chain with the transition from a solid to a fluidized state.

It was shown that the capillary bridges which are located at the positions $L_{x} / 4$ and $3 L_{x} / 4$ experience the largest force within the chain and therefore are the first

2. The dilation can easily be measured in simulations and a first rough estimate revealed $\Delta h_{f} / d \approx 0.2$. 
ones to rupture. This was in perfect agreement with the observation of shear bands at the same position in simulations. Additionally, it was shown that the fluidization threshold predicted by the model is in good qualitative agreement with the values observed by simulations. This is true for the capillary bridge force, $F_{B}$, as well as of the width of the system, $L_{x}$ and also the limit of $s_{\text {crit }} \rightarrow 0$. This is remarkable because the thin-thread model, is known from chapter 4 to show deviations from the minimal capillary model especially in the regime where the particle density is high and contact forces become important.

The limitations of the theoretical approach were clearly addressed and discussed. It was suggested that the functional dependence of the fluidization threshold on the critical rupture separation seems to be caused by an increase of the binding energy and thus cannot be captured in a purely force based model. The same is true for a gravitational energy barrier, which was qualitatively shown to originate from the potential energy of the particles.

It was shown in this chapter that some basic features of the complex dynamics emerging at the fluidization transition in sinusoidally sheared granular matter can be understood on the basis of a very simple one-dimensional model. 


\section{Unstable Kolmogorov flow in granular matter}

A solid and a fluidized state can be clearly distinguished in the phase diagram of sheared wet granular matter, as shown in the previous chapter. For the fluidized state, a snapshot was presented which showed the initial formation of a shear band in agreement with the simple theoretical model. The emerging dynamics of this fluidized state at later stages is studied in the present chapter, employing some standard tools known from nonlinear dynamics. While the dynamical states discussed in the previous chapters were suggestive of phases in equilibrium thermodynamics, here we will see the unfolding of enormous complexity, which eludes any such simple description.

\subsection{Introduction}

The beginning of nonlinear dynamics (in popular science often simply called chaos theory) is connected with well-known scientists such as H. Poincaré, J. Hadamard, A. N. Kolmogorov and many others. One of the most popular ideas from this field, which was widely recognized outside the scientific world also, was termed by E. Lorenz as the 'butterfly effect' (Robert and Rosier, 2001); the sensitive dependence on initial conditions. With an approximation of the Navier-Stokes equation he derived a set of differential equations (Lorenz, 1963; Ruelle and Takens, 1971; Tucker, 1999; Stewart, 2000) which have become a textbook example of a non-linear system.

One of the main outstanding problems in nonlinear dynamics is a detailed understanding of turbulence (Armbruster et al., 1996). In a classical approach to that problem a simple unbounded periodic flow may be considered, which is caused by a spatially varying and periodic but time-independent external forcing. This was suggested by Kolmogorov and thus is commonly referred to as the Kolmogorov flow (Platt et al., 1991; Zhang and Frenkel, 1998). At small Reynolds numbers (ratio of inertial forces to viscous forces (Platt et al., 1991)) the flow is laminar. For Reynolds numbers larger than the critical Reynolds number the flow is known to become linearly unstable, showing a stationary state including the formation of eddies. This was first shown by Meshalkin and Sinai (1961). At even larger Reynolds 
numbers the inverse cascade appears in the turbulent flow, where energy is successively transferred to larger spatial scales (Kraichnan, 1967; Green, 1974). Since then, the Kolmogorov flow has been the source of a reasonable amount of interest in the literature (see, for example, She, 1987; Dubrulle and Frisch, 1991; Gama et al., 1991; Arnold and Khesin, 1992; Legras and Villone, 2005; Perlekar and Pandit, 2010) (and all articles citing Meshalkin and Sinai, 1961). Kolmogorov flow is also accessible to experiments, as was shown for example in a soap film (Burgess et al., 1999).

Numerical simulations of wet granular matter, that is driven by a Kolmogorov flow are shown in this chapter. The typical cosine-shaped forcing of the Kolmogorov flow was already studied in wet granular matter (see, for example, Schulz et al., 2003; Schulz and Schulz, 2006), although with a different perspective compared to the present study. Here an instability is reported which is in some aspects reminiscent of the one in a classical fluid. With the help of the bifurcation diagram three different spatio-temporal patterns which emerge in the system are distinguished. This is followed by studying the bifurcations itself and finally presenting some typical Poincaré recurrence maps as obtained by simulations.

\subsection{Bifurcation diagram}

By means of molecular dynamics simulation the behavior of granular matter under a shear force which varies sinusoidally in space is studied. This is schematically shown in Fig. 2.7. To this end, event-driven simulations of three-dimensional elastic hard spheres are performed using the thin-thread model to mimic the effect of capillary bridges.

A minimal driving force, $F_{\text {scrit }}$, is required to overcome the attractive interaction of the capillary bridges and fluidize the material. This was shown in the phase diagrams of Fig.7.5. As before, the shear force is acting in the $y$-direction of the system and it is expected that the particles (in the fluidized regime) simply follow the applied shear force. However, as can be seen in Fig.8.1 the dynamics of the system transfers significant parts of the motion from the $y$ to the $x$ direction. This is very reminiscent of the classical Kolmogorov flow instability (see, for example, Burgess et al., 1999). The flow perpendicular to the driving indicates the deviation from laminar flow. Therefore it is suitable to use the kinetic energy, $E_{\perp}$, which is involved in this motion as an 'order parameter', to discriminate different flow regimes. $E_{\perp}$ measures to some extent the chaotic activity of the system. For different amplitudes of the driving shear force, $F_{\mathrm{smax}}$, different flow regimes (blue shaded areas in Fig.8.2 (a)) are observed. The circles in the diagram Fig.8.2(a) show respective minimum and maximum values of $E_{\perp}$. For driving forces slightly larger than $F_{\text {scrit }}$ a state is found which will be denoted as oscillatory state. As shown in Fig.8.1(top) this is a state where initially a shear band is formed, but which becomes periodically unstable leading to a swirl in the system. At $F_{S_{\max }} / F_{\text {scrit }} \gtrsim 1.9$ the swirl state becomes a stable stationary state. For even larger driving forces, $F_{s_{\max }} / F_{\text {scrit }} \gtrsim 4.3$, the swirls 
undergo periodic breathing-like size changes (Fig. 8.1 bottom). The time-evolution of the kinetic energy $E_{\perp}$ is shown in Fig.8.2(b) for the three different states: oscillatory, stationary and breathing-like (from bottom to top). There it can be observed that the oscillatory and the breathing-like state are qualitatively different: in the breathing-like state the energy $E_{\perp}$ oscillates only around a stationary value while in the oscillatory state $E_{\perp}$ periodically almost vanishes.
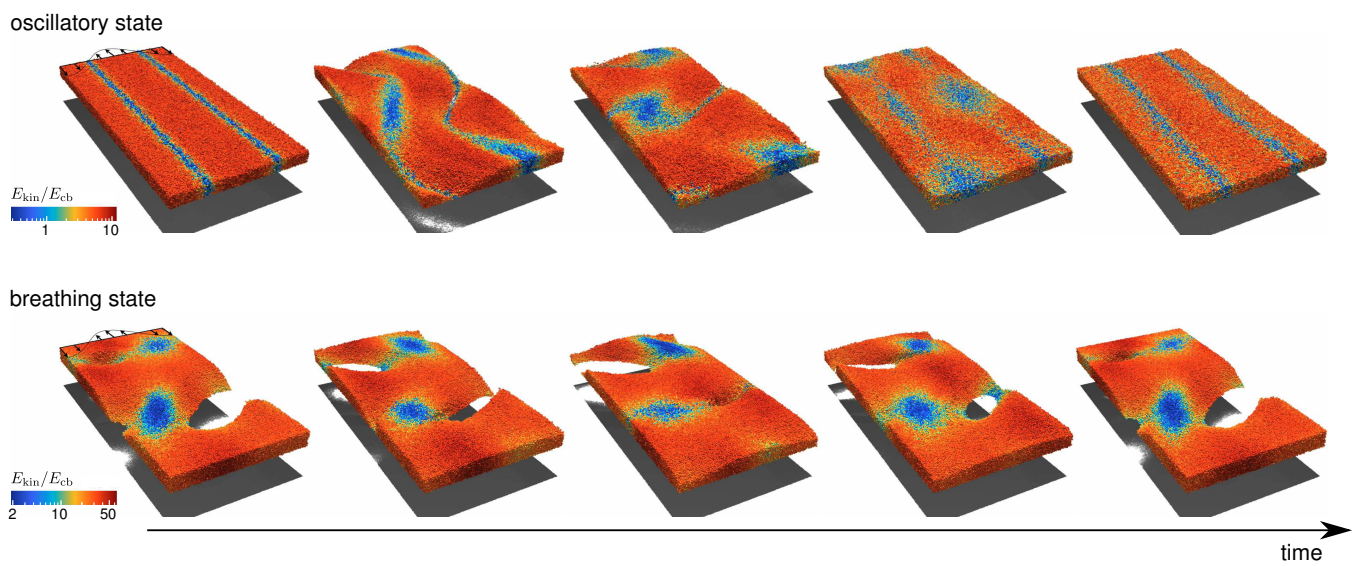

Fig. 8.1 Snapshots illustrating the time evolution (top: oscillatory state; bottom: breathing state) of $2.15 \cdot 10^{5}$ elastically colliding, monodisperse, wet spheres confined in a box with periodic boundary conditions in the two lateral directions. The system size is 100 particle diameters in width, 200 in length and 12.5 in height and the color of each particle indicates its kinetic energy. The flow is driven by a cosineshaped shear force sketched in the left-most images. The maximum driving force is $F_{\mathrm{smax}} / F_{\mathrm{scrit}}=1.26$ (oscillatory) and $F_{\text {smax }} / F_{\text {scrit }}=5.19$ (breathing-like). A video which shows the oscillatory, breathinglike as well as the stationary state can be viewed online under http://dx.doi.org/10.1063/1. 3202616.1.

The qualitative features of these flow patterns appear to be robust to changes of system size and numerical implementation of the wet granulate. They have also been observed for system sizes up to $1.72 \cdot 10^{6}$ particles, and in two-dimensional Kolmogorov flows where capillary bridges are modeled based on the minimal capillary model. The basic flow instability is even present in $d r y$ granular matter, where particles interact solely by inelastic hard-core collisions, although the morphology of the swirls strongly differs in that case due to the absence of attractive forces.

\subsection{Phase space plots - Limit cycles}

The kinetic energy $E_{\perp}$ of the particle motion perpendicular to the driving force served as the 'order parameter' indicating something like the chaotic activity of the system. In contrast to that, the kinetic energy $E_{\|}$of the particle motion parallel to the driving force indicates the tendency to flow in the direction of the applied shear force. Originally the system evolves in a $2 D N_{p}$ dimensional phase space, where $D$ is the dimension and $N_{p}$ is the number of particles. For the system presented in Fig.8.1 


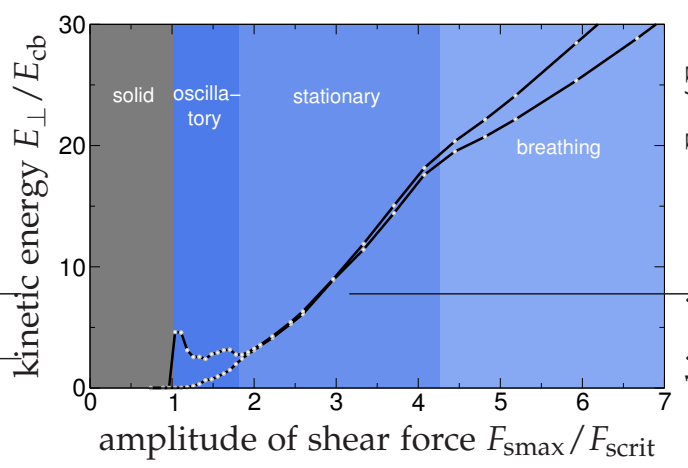

(a)

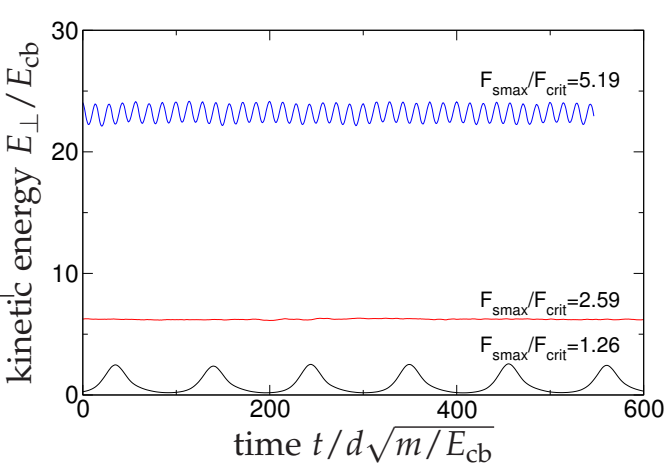

(b)

Fig. 8.2 (a) Bifurcation diagram for different driving forces $F_{\mathrm{smax}}$ normalized by the critical driving force, $F_{\text {scrit }}$, necessary to fluidize the material. The gray shading and the different shades of blue represent solid material and different flow regimes respectively. (b) The time evolution of the kinetic energy of the particle motion perpendicular to the driving force, $E_{\perp}$, is shown for the three different states oscillatory (black), stationary (red) and breathing-like (blue). The system size is 100 particle diameters in width, 200 in length and 12.5 in height and contains $2.15 \cdot 10^{5}$ monodisperse, wet spheres. The capillary interaction is characterized by the capillary bridge energy $E_{\mathrm{cb}} / m g s_{\mathrm{crit}}=6.0$ and a critical rupture separation $s_{\text {crit }} / d=0.0625$.

this leads to a $1.29 \times 10^{6}$ dimensional phase space. In the present section the system shall be studied in the phase space which is spanned by $E_{\|}$and $E_{\perp}$ and therefore is only two-dimensional. The trajectories of the system in this phase space are shown in Fig. 8.3. The different graphs are for different shearing amplitudes, $F_{\text {smax }}$, starting slightly above $F_{\text {scrit }}$ in the oscillatory regime with a limit cycle oscillating between shear bands and swirls (a-e). The states (d) and (e), where $E_{\perp}$ is significantly nonzero all the time, are states in which the shear band is never completely straight and parallel to the driving but always shows a deformation in the direction perpendicular to the applied shear force. The phase space trajectory of a stationary state can be seen in (f) and is simply a fixed point. The last two graphs $(\mathrm{g}, \mathrm{h})$ show the breathinglike regime as a limit cycle oscillating around an unstable fixed point, where the size of the voids (and similarly the swirls) in the system is periodically changing. Both the transition from the oscillatory to the stationary regime as well as the transition from the stationary to the breathing-like regime are clearly identified as supercritical Hopf bifurcations (Chen and Price, 1996; Chen et al., 2003; Kuznetsov, 2006, and references therein).

If the driving force approaches $F_{\text {smax }} / F_{\text {scrit }} \rightarrow 1$, a kink (indicated with an arrow) becomes visible in the limit cycle of Fig. 8.3(a) and (b) which is not present for larger driving amplitudes (c-e). In this limit, the origins of these graphs (which indicate a solid state) still seem to be hyperbolic fixed points. Thus a qualitative change must occur when the system goes from the fluidized to the solid state. In the solid state the origin can no longer be a hyperbolic fixed point but must act as an attractor such that the system ends up in the origin for different initial conditions of the system. 


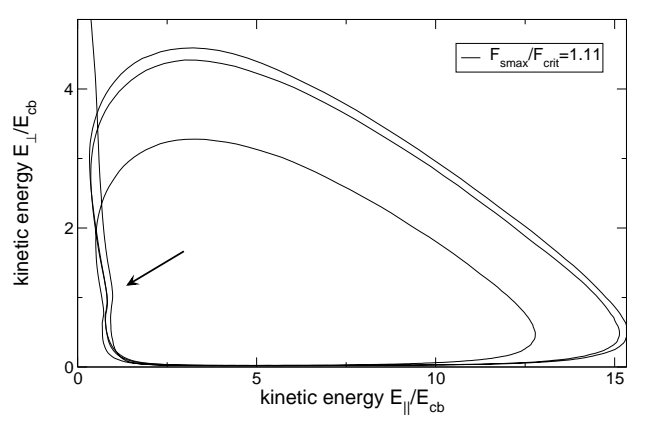

(a) Driving force: $F_{\text {smax }} / F_{\text {scrit }}=1.11$

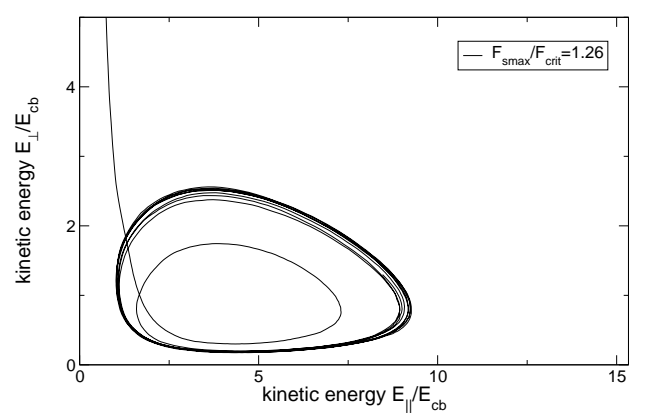

(c) Driving force: $F_{\mathrm{smax}} / F_{\mathrm{scrit}}=1.26$

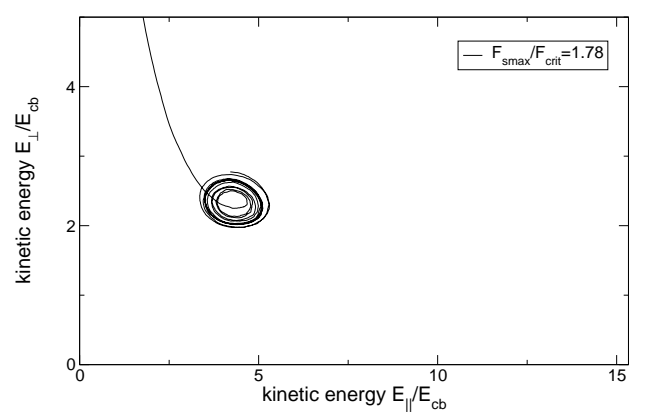

(e) Driving force: $F_{\mathrm{smax}} / F_{\text {scrit }}=1.78$

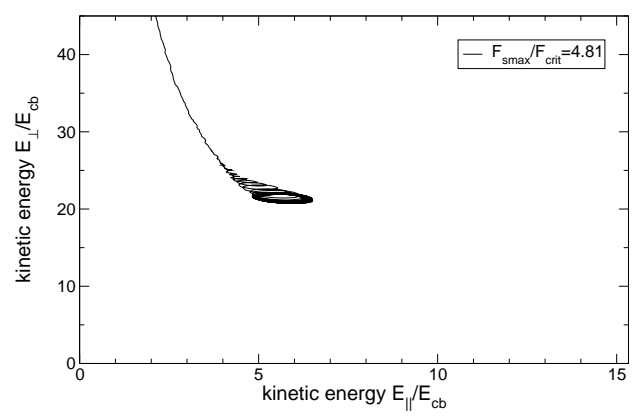

(g) Driving force: $F_{\text {smax }} / F_{\text {scrit }}=4.81$

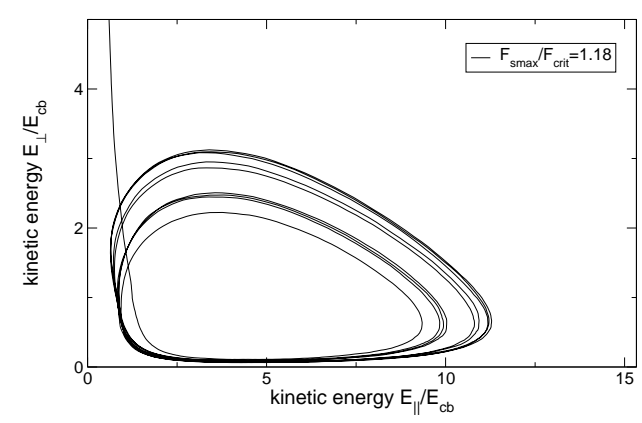

(b) Driving force: $F_{\mathrm{smax}} / F_{\mathrm{scrit}}=1.18$

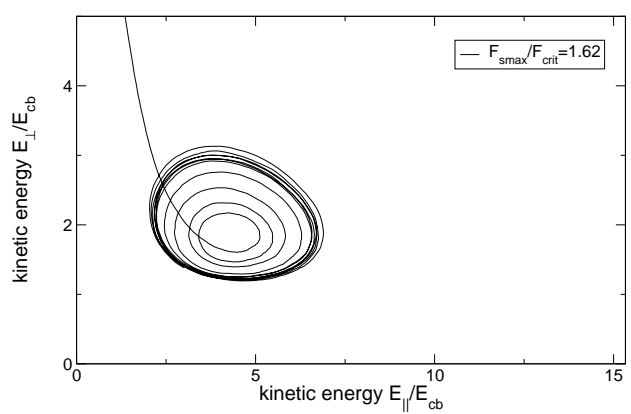

(d) Driving force: $F_{\mathrm{smax}} / F_{\text {scrit }}=1.62$

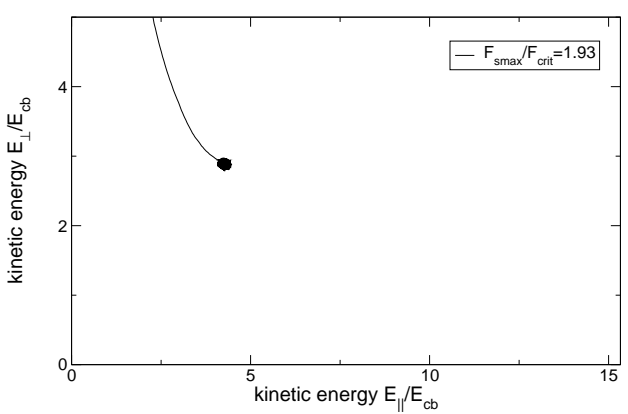

(f) Driving force: $F_{\text {smax }} / F_{\text {scrit }}=1.93$

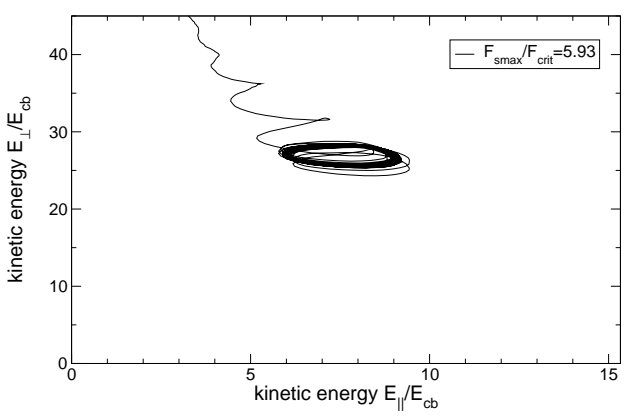

(h) Driving force: $F_{\mathrm{smax}} / F_{\text {scrit }}=5.93$

Fig. 8.3 Phase space plots in the plane spanned by the kinetic energy of the particle motion parallel, $E_{\|}$, and perpendicular, $E_{\perp}$, to the driving. Limit Cycles are observed in the oscillatory (a-e) and breathinglike $(\mathrm{g}, \mathrm{h})$ state while the stationary state is visible as a fixed point $(\mathrm{f})$. The transition between the different states is clearly identified as a supercritical Hopf bifurcation. For system parameters see Fig.8.2. 


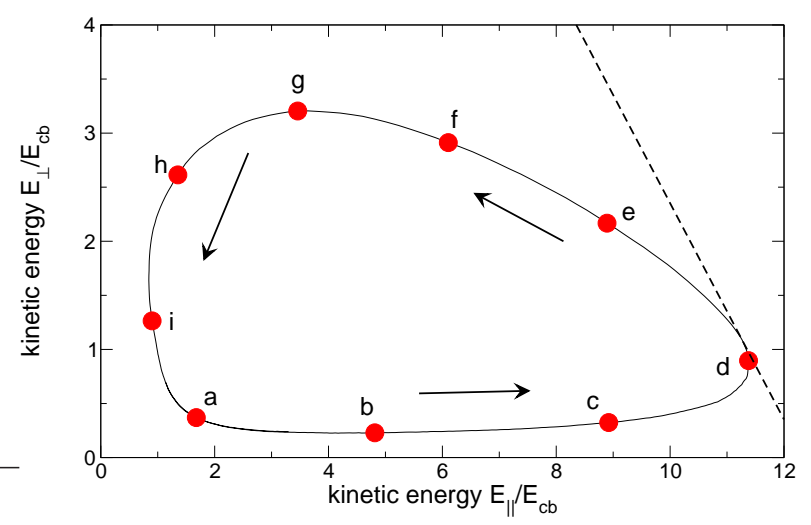

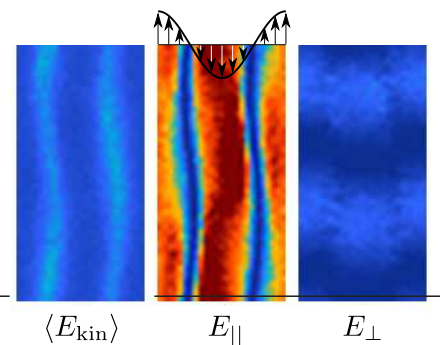

(a)

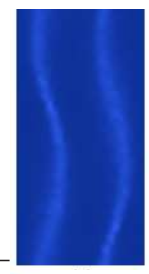

$\left\langle E_{\text {kin }}\right\rangle$

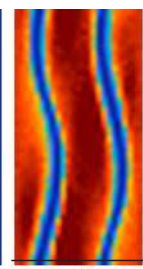

$E_{||}$

(d)

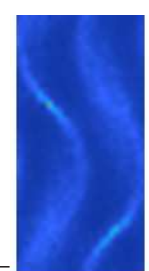

$\left\langle E_{\text {kin }}\right\rangle$

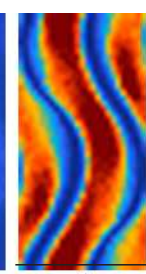

$E_{\|}$

(g)

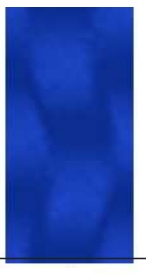

$E_{\perp}$

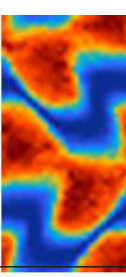

$E_{\perp}$

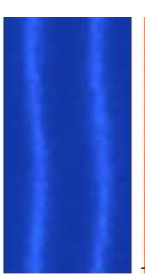

$\left\langle E_{\text {kin }}\right\rangle$

$E_{\|}$

(b)

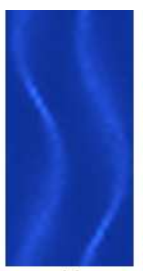

$\left\langle E_{\text {kin }}\right\rangle$

$E_{\|}$

(e)

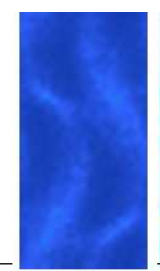

$\left\langle E_{\text {kin }}\right\rangle$

$E_{\|}$
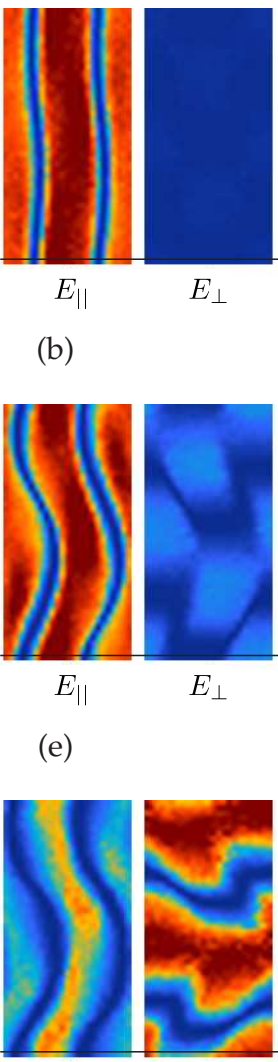

(h)
$E_{\perp}$

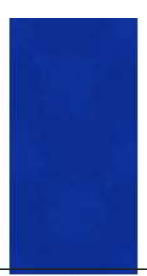

$E_{\perp}$

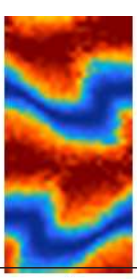

$E_{\perp}$

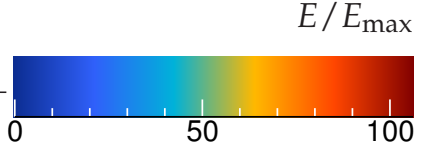

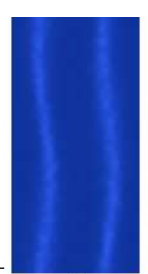

$\left\langle E_{\text {kin }}\right\rangle$

$E_{\|}$

(c)

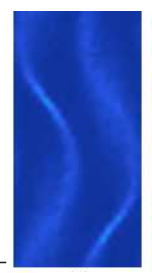

$\left\langle E_{\text {kin }}\right\rangle$

$E_{\|}$

(f)

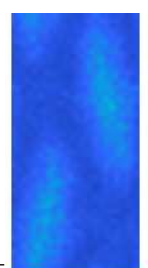

$\left\langle E_{\text {kin }}\right\rangle$

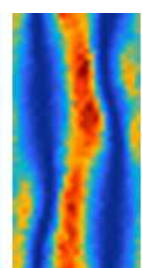

$E_{\|}$

(i)

Fig. 8.4 Top: One cycle (solid line) for an oscillatory state $\left(F_{\mathrm{smax}} / F_{\text {scrit }}=1.18\right)$ in the phase space is shown. The arrows denote the direction of increasing time. The red dots mark the positions at which the snapshot as labeled is taken and is presented in subfigure (a) to (i). For each snapshot the kinetic energy of the random particle motion, $\left\langle E_{\text {kin }}\right\rangle$, the kinetic energy parallel, $E_{\|}$, and perpendicular, $E_{\perp}$, to the driving force is shown. The colors are chosen in each image to enhance the contrast, but is unique among the different types of energy within a snapshot, where red denotes large and blue low kinetic energy. The dashed line in the phase space plot denotes a constant total energy, it shows that from (a) to (d) injection of energy dominates and from (d) to (i) dissipation dominates. For further description refer to the text. The system parameters are given in the caption of Fig.8.2. The maximum kinetic energies are as follows: (a) $E_{\max } / E_{\mathrm{cb}}=4.3$, (b) $E_{\max } / E_{\mathrm{cb}}=31.0$, (c) $E_{\max } / E_{\mathrm{cb}}=106.1$, (d) $E_{\max } / E_{\mathrm{cb}}=178.1$, (e) $E_{\max } / E_{\mathrm{cb}}=121.7$, (f) $E_{\max } / E_{\mathrm{cb}}=63.2$, (g) $E_{\max } / E_{\mathrm{cb}}=22.2$, (h) $E_{\max } / E_{\mathrm{cb}}=11.3$ and (i) $E_{\max } / E_{\mathrm{cb}}=3.5$. 
For a better understanding of the dynamics ${ }^{1}$ it is interesting to look at the energy in the system in spatial resolution. In small subsets of the system, 1) the kinetic energy of the random particle motion, $\left\langle E_{\text {kin }}\right\rangle=\frac{1}{2} m\left(\left\langle\vec{v}^{2}\right\rangle-\langle\vec{v}\rangle^{2}\right)$, 2) the kinetic energy parallel, $E_{\|}$, and 3$)$ the kinetic energy in the direction perpendicular to the driving, $E_{\perp}$, are monitored. The spatial extension of each subset is four particle diameters in each lateral direction and ranges over the full height of the system. Thus each subset contains on average approximately 170 particles and the whole system is divided into 25 subsets in the $x$ and 50 subsets in the $y$ direction. This is shown in Fig. 8.4 for an oscillatory state, where nine snapshots (a)-(i) are taken along one cycle to illustrate the oscillation between the shear band and the swirl state. On top of the snapshots the corresponding limit cycle is shown (solid curve) and the red circles denote the point where the respective snapshot was taken. The maximum shear force is $F_{\text {smax }} / F_{\text {scrit }}=1.18$ and thus corresponds to the simulation shown in Fig. 8.3(b). The color in the snapshots code the respective kinetic energy on a linear scale, where blue is low and red is high kinetic energy. The color code is the same for all three energies in a single snapshot, but changes along with the snapshot in order to best reveal the details. The corresponding color scale is shown in (c) on top of the snapshot.

In Fig. 8.4(a-c) the cycle starts with the formation of a shear band. It can be seen in all three snapshots (in $E_{\|}$) that the shear band never becomes completely straight. This causes a significant non-zero kinetic energy $E_{\perp}$, which is visible in (a) or in the phase space diagram on top of the snapshots. The increase of $E_{\|}$from (a) to (c) that is visible in the phase space diagram indicates that the shear bands are further accelerated once formed and thus their velocity is increasing. This means that more energy is injected into the system than can be dissipated. Energy is of course only injected by the shear force in the direction parallel to the shear force, $E_{\|}$. The kinetic energy in the other directions cannot be increased directly through the driving but needs a coupling mechanism, such as particle collisions. No significant change can be observed in the kinetic energy of the random motion $\left\langle E_{\text {kin }}\right\rangle$ throughout the cycle. Additionally, it can be seen that most of the kinetic energy is not in the random motion of the particles, $\left\langle E_{\mathrm{kin}}\right\rangle$, but in the directed flow either parallel or perpendicular to the driving. The kinetic energy $\left\langle E_{\text {kin }}\right\rangle$ even seems to be small compared to the

1. The center of mass is subject to a random motion because the mean force acting in the system depends on the distribution of particles. This can easily be envisaged if the system only contains one particle, such that the center of mass moves along with this particle. Assuming zero particle velocity initially the particle (as well as the center of mass) is accelerated to a certain direction parallel to the shear force. If the particle is additionally moving with a constant velocity perpendicular to the driving (which cannot be changed by the shear force and such remains constant) then the particle periodically changes its velocity. If many particles are in the system there will unavoidably be a small but non-zero force acting on the center of mass leading to a fluctuation of its position. This gives rise to a spurious diffusion in particle simulations of the Kolmogorov flow (as reported in Mansour et al., 1999), which corrupts statistical properties of the flow. This spurious diffusion is not relevant here, however, because the time-scale on which the fluctuations affect the macroscopic motion are large compared to the time-scales simulated here. 
capillary bridge energy $E_{\mathrm{cb}}$. This is a non-trivial observation: it means that the random motion of the particles must be cooled more effectively by the capillary bridges than the directed flow. A possible explanation for this could be as follows. The energy dissipation through the capillary bridge occurs in the center of mass system of the two particles which are connected via a liquid bridge. Hence, if the kinetic energy in their motion relative to each other is larger than the capillary bridge energy, $E_{\mathrm{cb}}$, the capillary bridge will rupture as soon as the surface separation reaches the critical rupture distance $s_{\text {crit }}$. This means that the velocity of the particles relative to each other determines if the capillary bridge can be ruptured. Assuming, for example, a freely cooling, dilute granular gas with a Gaussian velocity distribution (cf. appendix A) of mean kinetic energy $\left\langle E_{\text {kin }}\right\rangle$ without correlations between particle velocities prior to their collision. Then the relative velocities can be assumed to be distributed according to the same distribution. Thus the probability whether the capillary bridge ruptures after the preceding collision or not can be calculated. In (Ulrich et al., 2009a) it was shown that for a mean kinetic energy which is larger than the capillary bridge energy it can be assumed that every collision leads to the rupturing of corresponding liquid bridge. This leads to dissipation of the kinetic energy $E_{\mathrm{cb}}$ in each collision. Turning back to the shearing scenario the situation changes significantly. Even if $E_{\|}$or $E_{\perp}$ show values larger than $E_{\mathrm{cb}}$ this does not mean that the relative velocities of adjacent particles are large as well. This is because both kinetic energies indicate a directed flow which itself is especially characterized by small relative velocities of adjacent particles. This means that even if $E_{\|}$or $E_{\perp}$ is larger than $E_{\mathrm{cb}}$ not every particle collision will lead to a rupture of the corresponding capillary bridge. Therefore, even if the kinetic energy $E_{\|}$or $E_{\perp}$ in the directed flow is the same as the kinetic energy $\left\langle E_{\mathrm{kin}}\right\rangle$ in the random motion, there will be much less dissipation. This finding can be summarized as follows: As the energy dissipation through capillary bridges occurs in the center of mass of the two particles, dissipation is more effective for random motion than for directed flow. The capillary bridges therefore act as a selective mechanism which suppresses random motion and supports the formation of directed flow.

Turning back to the limit cycle shown in Fig.8.4 (d) to (i). Where the maximum value of $E_{||}$is reached it can be seen, that the deformation of the shear band increases such that energy is transferred from $E_{\|}$to $E_{\perp}$. $E_{\perp}$ reaches its maximum value in (g) where it becomes comparable to $E_{\|}$. This can be seen from approximately similar colors in the snapshots, which indicates that the swirl is now at its most pronounced. The swirl may be difficult to see in these snapshots due to the loss of direction information). The dashed line in the phase space plot on the top of the figure denotes constant total energy, which is $E_{\|}+E_{\perp}=$ const. Therefore it can be seen that energy injection was dominating over dissipation in the regime from (a) to (d). However, now in the regime from (d) to (i) the dissipation is strongly increasing and dominating against energy injection, leading to a decrease in the total energy. In snapshot (h) it can be seen that most energy is stored in $E_{\perp}$. And finally in (i) the bending of the shear band has almost vanished so that no more energy is transferred 
from $E_{\|}$to $E_{\perp}$ but accumulates in $E_{\|}$. The cycle then starts again. It is noted that the snapshots are not equidistant in time. In fact the energy injection from (a) to (d) takes much longer than the stage of high dissipation from (d) to (i).

It seems that the main mechanism to support the oscillation between the shear band and the swirl is the energy injection into the mode $E_{\|}$, which then transfers the energy into the mode $E_{\perp}$. This seems to be accompanied by an increase in dissipation until $E_{\perp}$ has decayed to very small values again.

\subsection{Poincaré maps}

The dynamics of a system can be well captured by means of a Poincaré recurrence map (Marwan et al., 2007; Robinson and Thiel, 2009). A Poincaré section can be defined for the present system (following the work by Lorenz (1963)) as the maximum value of $E_{\perp}$ during each cycle. The Poincaré maps which result for different external driving forces are shown in Fig. 8.5, where (a) to (f) show an oscillating, (g) a stationary and (h) a breathing-like state. The red lines are the bisecting lines. A fixed point is found as the intersection between this bisecting line and the Poincare recurrence map. It is stable if the absolute value of the slope of the Poincare map at the fixed point is smaller or equal to one. The red point in each figure (indicated for example in (c)) denotes the stable fixed point in which the system ends up. The system size, that was used in these simulations was reduced to $L_{x} / d=48$ and $L_{y} / d=96$ and contains $2.86 \times 10^{4}$ particles. The reduction of the system size is necessary since many simulations of the same system with different initial conditions have to be performed in order to obtain the Poincaré map. The corresponding phase space plots are not shown here again, as they do not provide any additional insight.

The data points in the one-dimensional Poincaré maps fall on curves reminiscent of a unimodal map. For shearing forces well above the fluidization threshold, $F_{\text {scrit, }}$ the maps are reminiscent of a logistic map at small enough energies, as can be seen in Fig. 8.5 (b) to (f). It is best seen for example in (c) in the range between $\max \left(E_{\perp}\right)_{n} / E_{\mathrm{cb}}=1.5$ and 3. Additionally, in (c) it can be seen that at the lowest energies, $\max \left(E_{\perp}\right)_{n} / E_{\mathrm{cb}}$, the value comes close to the bisecting line. If one extrapolates the Poincaré map to the bisecting line (blue line in (c)) another fixed point is obtained (marked by an arrow). Because the slope of the Poincare section at this point seems to be larger than one this fixed point is unstable. The green line in (c) is straight with slope negative one through the fixed point. It can be seen that the Poincaré map seems to be very close to this value but due to the scattering cannot be clearly justified. If the slope is slightly larger than one period doubling should be visible. The limit cycle presented in Fig. $8.3(\mathrm{~b})$ suggests that this indeed could be the case. A larger system could possibly be studied in order to reduce the scattering in the Poincaré maps. If the external driving is lowered and comes close to the fluidization threshold the corresponding return map shown in (a) shows even more scattering around the stable fixed point. This indicates increasingly complex 
dynamics when approaching the solid state. The appearance of the scattering does seem reasonable, since a kink occurs in $E_{\|}$of the limit cycles as was indicated with an arrow in Fig.8.3(a). This is most probably caused by the attraction of the solid state attractor that superposes with the attractor of the oscillatory state. An effective dynamics with more than two independent variables is then necessary to obtain a faithful description. As a possible third parameter the potential energy of the particles is suggested here. This is because in simulations of the oscillatory state first observations were made that it changes during a cycle.

The stationary state in the bifurcation diagram is reached in Fig. $8.5(\mathrm{~g})$, where the Poincaré recurrence map simply appears to be uniform, so that any initial value immediately ends up in the stationary state. The same is basically true for the breathing-like state in Fig. 8.3(h). Additionally, two fixed points seem to appear at the intersection of the red line and the numerically obtained Poincaré map. One fixed point is clearly unstable as the slope of the Poincare map is larger than one. The second fixed point at the largest energies seems to be slightly attractive, but it is not the final stable fixed point in which the system ends up.

The complex dynamics of the wet granular matter driven by a cosined-shaped shear force seems to be determined by a Poincaré recurrence map. This is remarkable since the phase space has been reduced from approximately $1.7 \times 10^{5}$ dimensions to a two-dimensional phase space spanned by $E_{\|}$and $E_{\perp}$. This also means that the spreading of the limit cycles in their phase space which is visible in Fig. 8.3 is caused not only by erratic fluctuations but also by deterministic chaotic motion.

\subsection{Summary and Outlook}

In this chapter simulations of wet granular matter driven by a cosine-shaped shear force were presented. This is commonly known as Kolmogorov flow and was originally introduced as a simple model system to study flow instabilities in the NavierStokes equation. In these simulations a rich set of dynamic instabilities could be found with three different regimes clearly distinguishable. The first is an oscillatory regime where the system oscillates between a shear band and a swirl state. This is followed at larger driving forces by a stationary state resulting in the formation of a dynamically stable pattern with swirls. Due to the attractive forces even voids form in the center of the elliptic points. The third and last regime reported here is called breathing-like regime, since the size of the voids periodically changes. By presenting the limit cycles of these different states it was suggested that the transitions between the different regimes are supercritical Hopf bifurcations in both cases. The recurrence map was obtained for the Poincaré section defined as the maximum kinetic energy of the particles perpendicular to the driving, $E_{\perp}$; this map showed similarities with a logistic map in some ranges. This is remarkable as the original high-dimensional phase space of the system was reduced to only two dimensions, which is spanned by the kinetic energies parallel $E_{\|}$and perpendicular $E_{\perp}$ to the 
driving force. First indications were shown of a possible period doubling scenario. This deserves further investigation as it determines the route to more complex behavior.

The vanishing kinetic energy of the random motion in the oscillatory state suggests that the capillary bridges act as a selective mechanism which suppresses random motion and supports the formation of directed flow. Because the energy dissipation through capillary bridges occurs in the center of mass of the two particles linked by the bridge, arguments were given that dissipation occurs more effectively for random motion than for directed flow.

As the instability of the flow has also been observed in two-dimensional as well as dry Kolmogorov flow systems (both not presented here) it would be interesting to explore whether it is possible to observe this instability also in experiments. The stability analysis for the granular Kolmogorov flow system by using granular NavierStokes hydrodynamics is left for future work.

Significant parts of this chapter have been published in Roeller, Vollmer, and Herminghaus (2009). 


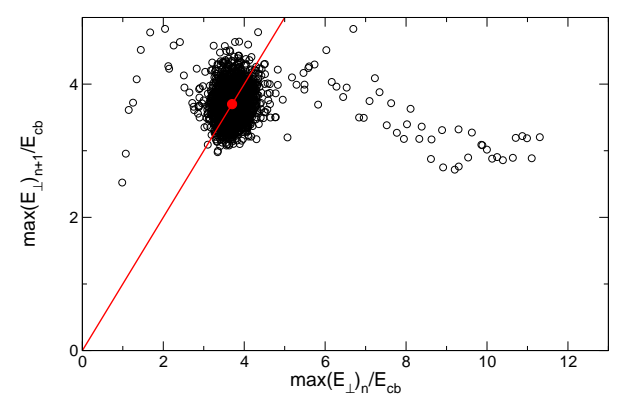

(a) Driving force: $F_{\text {smax }} / F_{\text {scrit }}=1.07$

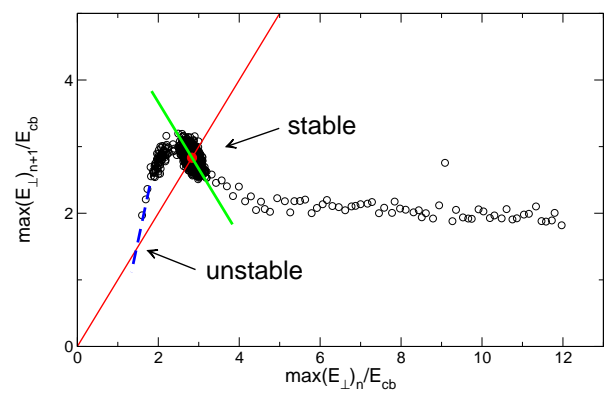

(c) Driving force: $F_{\text {smax }} / F_{\text {scrit }}=1.39$

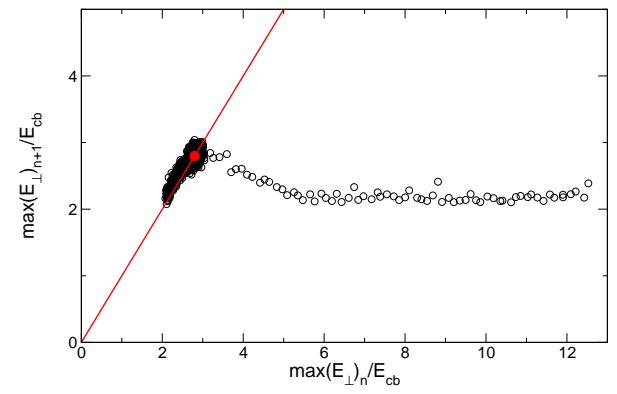

(e) Driving force: $F_{\text {smax }} / F_{\text {scrit }}=1.58$

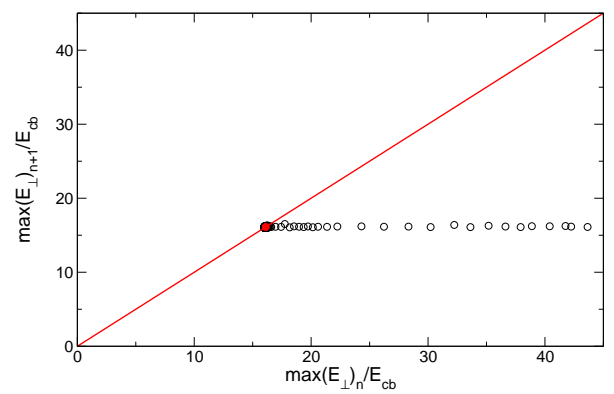

(g) Driving force: $F_{\text {smax }} / F_{\text {scrit }}=3.17$

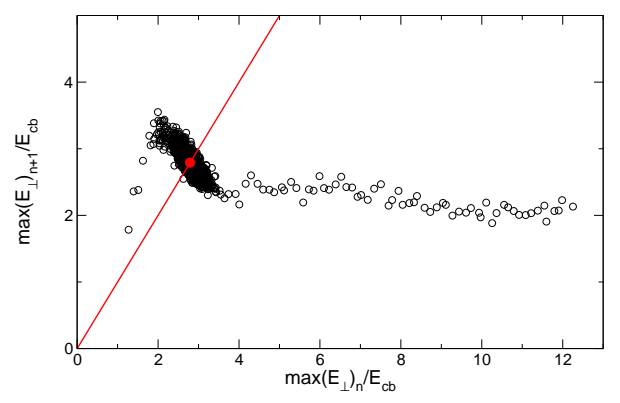

(b) Driving force: $F_{\mathrm{smax}} / F_{\mathrm{scrit}}=1.27$

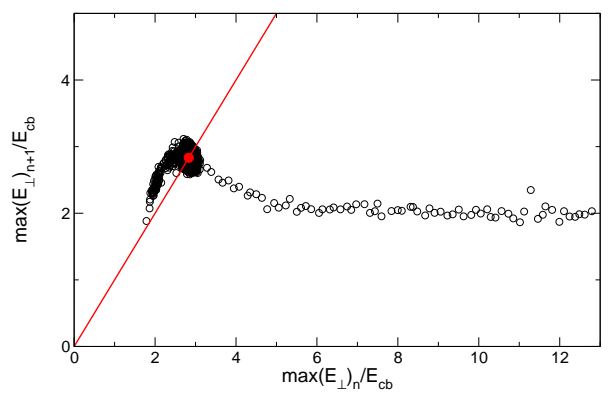

(d) Driving force: $F_{\text {smax }} / F_{\text {scrit }}=1.47$

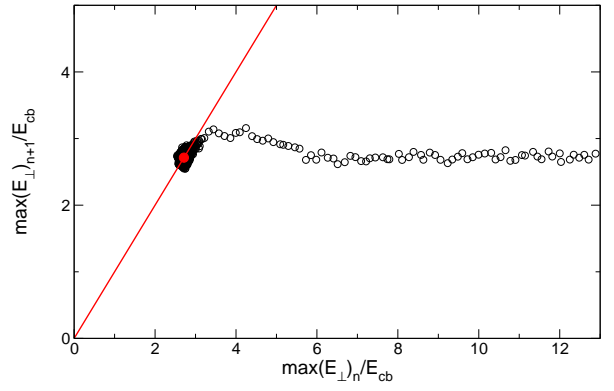

(f) Driving force: $F_{\text {smax }} / F_{\text {scrit }}=1.78$

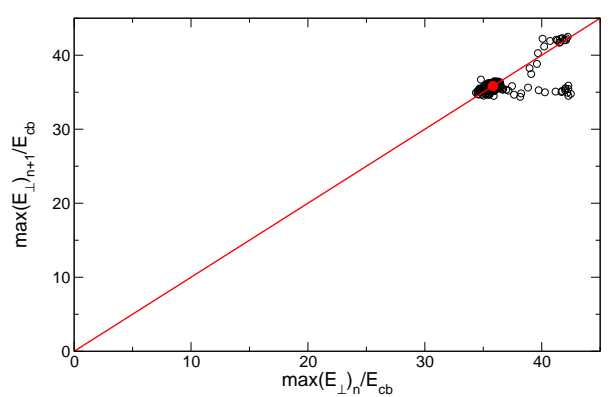

(h) Driving force: $F_{\mathrm{smax}} / F_{\text {scrit }}=7.52$

Fig. 8.5 The Poincaré recurrence map as obtained by simulations (black circles) defined by the maximum of $E_{\perp}$ in each cycle. It is shown for the oscillatory regime (a)-(f), a stationary state $(\mathrm{g})$ and a breathing state $(\mathrm{h})$. The red line is the bisecting line and the red circle shows the respective fixed point. The system contains approximately $2.86 \times 10^{4}$ elastic hard spheres in a system of 48 particle diameters in width, 96 particle diameters in length and 7.5 particle diameters in height. Other parameters are the same as noted in the caption of Fig.8.2. 


\section{Summary}

Wet granular matter is a useful system for studing the dynamics which emerge in systems far from thermal equilibrium. It is easily accessible by experiments as well as simulations. With the help of time- as well as event-driven molecular dynamics simulations, wet granular matter was studied in this thesis. The energy injection which is necessary to drive the system out of thermal equilibrium was done by two highly different sinusoidal driving mechanisms. The first one was sinusoidal shaking and was used from chapter 3 to 6 , whilst the second one was sinusoidal shearing and was used in chapters 7 and 8 . The interaction with the liquid in the system was modeled by the minimal capillary model and the thin-thread model, respectively. They account for the dissipation of energy such that the system can reach a steady state.

In the first part of this thesis sinusoidal shaking was employed enabling the system to reside in states which are very reminiscent of solids, fluids or gases known from systems in thermal equilibrium. In the spirit of equilibrium thermodynamics phase diagrams were obtained for two- as well as three-dimensional wet granular matter. It was shown by simulations that agitated wet granular matter shows two universal phase transitions which depend only on a few general intensive system parameters.

One is the solid-fluid transition which was shown to be mainly driven by the applied driving force, whilst the driving energy acts as a second order correction. A simple theoretical model which only assumes the balance of basic forces acting in the system showed good qualitative agreement with simulations at small driving energies. The model assumes that the equality of the mean free path between different layers of particles and the critical rupture separation to rupture a capillary bridge determines the solid-fluid transition. Furthermore, it predicts surface melting which was also observed in simulations. The second transition found in wet granular matter was between a fluid phase and the gas phase accompanied by a broad coexistence region. It was driven by the maximum kinetic energy of the driving wall. The transition occured if the maximum energy provided by a single collision with the wall was sufficient to rupture one capillary bridge. Additionally, by reducing the polydispersity, a solid-fluid transition was found, which was also determined by a critical driving energy and accompanied by a solid-fluid coexistence.

The study of the size of the gas bubble which emerged in the fluid-gas coexistence region revealed three different shapes: a circular gas bubble surrounded by fluid, a 
strip shape and a fluid droplet immersed in the gas phase. This situation reminded of the transitions between similar shapes which emerge in a two-dimensional Ising model. The transition between the two shapes in the equilibrium model gives rise for a first order phase transition and is driven by the minimization of the interfacial free energy. This similarity led to the suggestion of a quantity analogous to an interfacial energy to be present in wet granular matter. The interfacial energy was estimated in simulations by mechanically deforming the interface and was found to be in qualitative agreement with a simple theoretical estimate. A further study of the universality of the interfacial tension revealed a behavior in a three-dimensional simulation of the solid-fluid coexistence, which is reminiscent of Ostwald ripening. Ostwald ripening is know to be driven by the minimization of the interfacial free energy. From the reminiscence of the two effects it was deduced that an interfacial energy seemed to be present also in the case of the solid-fluid coexistence in the three-dimensional system.

A coexistence does not only occur in the horizontal direction of the system but can also occur in the vertical direction. This revealed a situation of density inversion which is reminiscent of the Leidenfrost effect known from classical liquids. The apparent reduction of heat conductivity results in a clear interface between the hot gas and the cold condensed plug which hovers on top of the gas. The plug undergoes a smooth oscillation with an oscillation frequency, which is well described by a simple theoretical model. It is the same oscillation frequency as in the case of a passive plug which hovers on top of the evaporated gas, where corrections due to excluded volume effects are taken into account. If a lateral gas exchange is introduced into the model, a Rayleigh-Taylor instability is predicted, this being reminiscent of bubbling in the Leidenfrost scenario for water. This is also oberserved in simulations and is in reasonable agreement with the theoretical prediction.

In the second part of this thesis, sinusoidal shearing was used to drive the wet granular matter. Phase diagrams were presented which showed a solid and a fluidized state. A simple theoretical model was presented which connected the stability of a single one-dimensional chain of particles with the transition observed in the simulations. Good qualitative agreement was found between the theoretical estimate and the simulation. The limitations of the theoretical model were discussed and arguments on the origin of the deviations were given.

This was followed by a study of the rich and complex dynamics emerging in the fluidized state in the sheared granular matter. In the wet granular matter a rich set of dynamic instabilities was found and studied with the standard tools known from non-linear dynamics. Three different regimes were found. The first one was called osciallatory regime, where the shear band periodically becomes unstable resulting in a swirl. At larger driving forces, a stationary state was found with a stationary swirl which becomes unstable at even larger driving forces in the sense that the voids undergo breathing-like size changes. Limit cycles were found in a two-dimensional phase space, which revealed a Hopf bifurcation at the transitions between the different regimes. Thus, the recurrence maps were presented. 
In summary, extensive numerical simulations were performed on wet granular matter in this thesis. This was possible due to the remarkably simple model (the minimal capillary model) which is used to describe the capillary interaction. A derivation of this model (the thin thread model) allowed for a significant performance increase when simulating. An amazingly rich set of dynamical behavior was found for wet granular matter using different driving methods. In many cases very simple models, which only incorporate little of the actual complexity of the problem, were able to predict some of their features. In spite of their simplicity, in many cases a good qualitative agreement between simulations and experiment is found.

Many phenomena were found in simulations which were reminiscent of behavior which is known from classical fluids. Thus, it can be concluded that wet granular matter is a complex fluid; complex, indeed is reminiscent of a fluid. 

Appendices 



\section{A. Velocity distribution of a wet granular gas}

The velocity distribution of a granular gas is of great interest when calculating transport coefficients like heat conductivity. For $d$ ry granular matter, velocity distribution as well as transport coefficients are standard knowledge which are readily available (Jenkins and Richman, 1986; Brey et al., 1998; Losert et al., 1999a; Brilliantov and Pöschel, 2004; Baskaran et al., 2008). We examine the velocity distribution of a wet granular gas by means of kinetic theory in a stationary state.

\section{A.1. The Boltzmann equation for a wet granular gas}

\section{A.1.1. Modeling a wet granular gas}

The Minimal Capillary Model (Herminghaus, 2005) is often used to model the interaction between particles of wet granular matter in simulations and theory. This model has been shown to be reasonably close to experiments (Willett et al., 2000). However, the formation and rupture of capillary bridges takes place at different spatial positions, which gives rise to hysteresis in the acting forces. Thus, it is unfortunately not possible to formulate a Boltzmann equation for such a system. Therefore, we use a modified version of the minimal capillary model, but preserve its most important feature, which is namely a constant loss of kinetic energy $E_{\mathrm{cb}}$ per rupture event (Zaburdaev et al., 2006; Fingerle et al., 2008). The capillary bridge is formed during the (fully elastic) collision of two particles. It ruptures instantaneously if the relative velocity $\vec{v}_{12}=\vec{v}_{1}-\vec{v}_{2}$ of the two particles 1 and 2 is greater than the critical rupture velocity $v_{\text {crit }}$ :

$$
\vec{v}_{12}>v_{\text {crit }}=\sqrt{\frac{2 E_{\mathrm{cb}}}{m}}
$$

where $m$ is the mass of each particle. If the relative velocity $\vec{v}_{12}$ is smaller than the critical rupture velocity of the capillary bridge, the particles remain together.

We neglect the case where particles stick together by restricting our considerations to a very dilute wet granular gas. Here dilute refers to a low packing fraction, $\frac{\pi}{6} \sigma^{3} n$, such that the particle volume is small compared to the volume of the gas and 
excluded volume effects do not have to be taken into account. Here $\sigma$ is the particle diameter and $n$ the number density. Furthermore, we investigate the wet granular gas in a regime where the temperature $T_{g}$ is much greater than the energy loss $E_{\mathrm{cb}}$ per rupture event. The temperature in $d$ dimensions is defined as the mean kinetic energy of the random particle motion:

$$
\left\langle\frac{1}{2} m V^{2}\right\rangle=\frac{d}{2} T_{g}
$$

with the local velocity $\vec{V}$ which is defined by

$$
\vec{V}(\vec{r}, t) \equiv \vec{v}(\vec{r}, t)-\vec{u}(\vec{r}, t),
$$

where $\vec{v}$ is the particle velocity with respect to the average velocity $\vec{u}$. With these two restrictions $\left(n \ll \frac{6}{\pi \sigma^{3}}\right.$ and $T_{g} \gg \frac{2}{d} E_{\mathrm{cb}}$ ) we are able to justify our assumption that every collision leads to a rupture of the created capillary bridge and we therefore can disregard sticking of particles.

\section{A.1.2. Collision rules}

The assumptions made above allow to write down a collision rule which contains both the collision, which is assumed to be elastic, and the energy loss due to the ruptured capillary bridge.

$$
E_{12}=E_{12}^{* *}-E_{\mathrm{cb}}
$$

where $E_{12}^{* *}$ and $E_{12}=\frac{1}{2}\left(\vec{v}_{12} \vec{\sigma}\right)^{2}$ is the energy of the relative motion of particle 1 and 2 before and after the collision respectively. $\vec{\sigma}=\frac{\vec{r}_{1}-\vec{r}_{2}}{\left|r_{1}-r_{2}\right|}$ is the unit vector pointing from the position of the center of particle $r_{2}$ to the center of particle $r_{1}$.

A straightforward calculation of the collision rule (A.4) leads to the following collision laws for identical particles:

$$
\begin{aligned}
& \vec{v}_{1}=\vec{v}_{1}^{* *}-\frac{\vec{\sigma}}{2}\left(\vec{v}_{12}^{* *} \vec{\sigma}\right)-\frac{\vec{\sigma}}{2} \sqrt{\left(\vec{v}_{12}^{* *} \vec{\sigma}\right)^{2}-2 E_{\mathrm{cb}}} \\
& \vec{v}_{2}=\vec{v}_{2}^{* *}+\frac{\vec{\sigma}}{2}\left(\vec{v}_{12}^{* *} \vec{\sigma}\right)+\frac{\vec{\sigma}}{2} \sqrt{\left(\vec{v}_{12}^{* *} \vec{\sigma}\right)^{2}-2 E_{\mathrm{cb}}}
\end{aligned}
$$

which are momentum-preserving. The notation with two asterisks means velocities before the collision, while post-collisional velocities do not have any additional label.

To devise the Boltzmann equation, we need to account for reverse collisions, which means that we calculate for a given post-collisional velocity the velocity of that particle right before the collision. This yields:

$$
\begin{aligned}
& \vec{v}_{1}^{* *}=\vec{v}_{1}-\frac{\vec{\sigma}}{2}\left(\vec{v}_{12} \vec{\sigma}\right)-\frac{\vec{\sigma}}{2} \sqrt{\left(\vec{v}_{12} \vec{\sigma}\right)^{2}+2 E_{\mathrm{cb}}} \\
& \vec{v}_{2}^{* *}=\vec{v}_{2}+\frac{\vec{\sigma}}{2}\left(\vec{v}_{12} \vec{\sigma}\right)+\frac{\vec{\sigma}}{2} \sqrt{\left(\vec{v}_{12} \vec{\sigma}\right)^{2}+2 E_{\mathrm{cb}}} .
\end{aligned}
$$


We can consider equations (A.7) and (A.8) as transformation of coordinates $\vec{v}_{1}^{* *}, \vec{v}_{2}^{* *} \rightarrow$ $\vec{v}_{1}, \vec{v}_{2}$ with jacobian $\mathrm{J}$ :

$$
\mathcal{J}=\left|\frac{\partial\left(\vec{v}_{1}^{* *} \vec{\sigma}, \vec{v}_{2}^{* *} \vec{\sigma}\right)}{\partial\left(\vec{v}_{1} \vec{\sigma}, \vec{v}_{2} \vec{\sigma}\right)}\right|=-\frac{1}{\sqrt{1+\frac{2 E_{\mathrm{cb}}}{\left(\vec{v}_{12} \vec{\sigma}\right)^{2}}}} .
$$

We additionally need

$$
\chi=\frac{\left|\left(\vec{v}_{12}^{* *} \vec{\sigma}\right) \vec{\sigma}\right|}{\left|\left(\vec{v}_{12} \vec{\sigma}\right) \vec{\sigma}\right|} \times \mathcal{J}=-\sqrt{1+\frac{2 E_{\mathrm{cb}}}{\left(\vec{v}_{12} \vec{\sigma}\right)^{2}}},
$$

where the first factor is the ratio of the lengths of the collision cylinders for the inverse and direct collisions (cf. Brilliantov and Pöschel, 2004). We finally arrive at the Boltzmann equation:

$$
\frac{\partial}{\partial t} f\left(\vec{v}_{1}, t\right)=I(f, f)
$$

with the velocity distribution function $f$ and the collision integral

$$
I(f, f) \equiv \sigma^{2} \int \mathrm{d} \vec{v}_{2} \int \mathrm{d} \vec{\sigma} \Theta\left(-\vec{v}_{12} \times \vec{\sigma}\right)\left|\vec{v}_{12} \times \vec{\sigma}\right|\left(\chi f\left(\vec{v}_{1}^{* *}, t\right) f\left(\vec{v}_{2}^{* *}, t\right)-f\left(\vec{v}_{1}, t\right) f\left(\vec{v}_{2}, t\right)\right) .
$$

\section{A.1.3. A useful property of the collision integral}

A feature of the collision integral which we will need below for further calculations is

$$
\begin{array}{r}
\int \mathrm{d} \vec{v}_{1} \psi\left(\vec{v}_{1}\right) I(f, f)=\frac{\sigma^{2}}{2} \int \mathrm{d} \vec{v}_{1} \mathrm{~d} \vec{v}_{2} \mathrm{~d} \vec{\sigma} \Theta\left(-\vec{v}_{12} \vec{\sigma}\right)\left|\vec{v}_{12} \vec{\sigma}\right| f\left(\vec{v}_{1}, t\right) f\left(\vec{v}_{2}, t\right) \\
\times\left[\psi\left(\vec{v}_{1}^{*}\right)+\psi\left(\vec{v}_{2}^{*}\right)-\psi\left(\vec{v}_{1}\right)-\psi\left(\vec{v}_{2}\right)\right]
\end{array}
$$

where $v_{i}(i=1,2)$ are the initial velocities and $v_{i}^{*}$ the velocities after the collision. A proof of this relation can be found in Brilliantov and Pöschel (2004, chapter 6.4).

\section{A.2. Velocity distribution expansion}

In contrast to systems in thermodynamic equilibrium, the velocity distribution for systems which are out of equilibrium are non-maxwellian. Furthermore, deviation of the velocity distribution from a gaussian is a measure how far the system is out of equilibrium (Speck and Seifert, 2004). Therefore, we calculate the first nontrivial expansion coefficient which describes the deviation from a gaussian for a homogeneous wet granular gas. 


\section{A.2.1. Normalized velocity distribution}

We consider the scaled form $F(\vec{c})$ of the velocity distribution function where the time dependence only occurs through the time-dependent average velocity $v_{0}$ (Esipov and Pöschel, 1997). That the velocity distribution function can be rescaled by the average velocity became clear while considering a freely cooling granular gas which is only slightly inelastic and therefore behaves at every point in time like a system in equilibrium (Esipov and Pöschel, 1997).

$$
F(\vec{c})=v_{0}^{d}(t) f\left(v_{0} \vec{c}, t\right),
$$

where $\vec{c} \equiv \vec{v} / v_{0}(t)$ is the scaled velocity and $v_{0}$ is the thermal velocity which is defined by the temperature

$$
T_{g}(t)=\frac{m}{2} v_{0}^{2}(t)
$$

which itself is defined by

$$
\frac{d}{2} n T_{g}(t) \equiv \int \mathrm{d} \vec{v} \frac{m V^{2}}{2} f(\vec{v}, t)
$$

Due to the temporary local equilibrium argument above, we can expect the velocity distribution to be close to a Maxwell distribution and therefore expand the scaled velocity distribution $F(\vec{c})$ to a complete set of orthogonal functions $\left\{L_{n}(x)\right\}$ :

$$
F(\vec{c})=\phi(\vec{c})\left[1+\sum_{n=1}^{\infty} a_{n} L_{n}\left(c^{2}\right)\right],
$$

where the leading term

$$
\phi(\vec{c})=\frac{1}{\pi^{d / 2}} \mathrm{e}^{-c^{2}}
$$

is a gaussian.

\section{A.2.2. Laguerre polynomial expansion}

A convenient choice for the orthogonal set of functions $\left\{L_{n}(x)\right\}$ are the Laguerre polynomials

$$
L_{n}^{(\alpha)}(x)=\frac{\mathrm{e}^{x} x^{-\alpha}}{n !} \frac{\mathrm{d}^{n}}{\mathrm{~d} x^{n}}\left(\mathrm{e}^{-x} x^{n+\alpha}\right)=\sum_{k=0}^{n}\left(\begin{array}{l}
n+\alpha \\
n-k
\end{array}\right) \frac{(-x)^{k}}{k !},
$$

where $\alpha=d / 2-1$. In the context of kinetic theory they are often called Sonine polynomials. The Laguerre polynomials up to order two for $d$ dimensions read:

$$
\begin{aligned}
L_{0}^{(d / 2-1)}(x) & =1 \\
L_{1}^{(d / 2-1)}(x) & =\frac{d}{2}-x \\
L_{2}^{(d / 2-1)}(x) & =\frac{1}{8}\left(2 d+d^{2}-(8+4 d) x+4 x^{2}\right) .
\end{aligned}
$$


The orthogonality relation reads

$$
\int_{0}^{\infty} \mathrm{e}^{-x} x^{\alpha} L_{m}^{(\alpha)}(x) L_{n}^{(\alpha)}(x) \mathrm{d} x=\left(\begin{array}{c}
n+\alpha \\
n
\end{array}\right) \Gamma(1+\alpha) \delta_{n m},
$$

where $\Gamma(x)=\int_{0}^{\infty} \mathrm{e}^{-t} t^{x-1} \mathrm{~d} t$ is the usual $\Gamma$-function and $\delta_{n m}$ the Kronecker delta. The inverse relation of (A.20) is

$$
x^{m}=\sum_{k=0}^{m}\left(\begin{array}{l}
m+\alpha \\
m-k
\end{array}\right) m ! L_{k}^{(\alpha)}(x) .
$$

We use Eq. (A.22) together with the orthogonality relation (A.21) and calculate moments of the velocity distribution function

$$
\left\langle c^{n}\right\rangle \equiv \int_{-\infty}^{\infty} c^{n} F(\vec{c}) \mathrm{d} \vec{c} .
$$

We find for the zeroth moment

$$
\begin{aligned}
\left\langle c^{0}\right\rangle & =\int F(\vec{c}) \mathrm{d} \vec{c} \\
& =1+\sum_{n=1}^{\infty} a_{n} \int_{-\infty}^{\infty} \mathrm{d} \vec{c} \phi(\vec{c}) L_{0}\left(c^{2}\right) L_{n}\left(c^{2}\right) \\
& =1
\end{aligned}
$$

where we expressed 1 by $L_{0}\left(c^{2}\right)$ and then used the orthogonality relation. We obtain analogously for the second moment

$$
\begin{aligned}
\left\langle c^{2}\right\rangle & =\int \mathrm{d} \vec{c}\left(\frac{d}{2}-L_{1}\left(c^{2}\right)\right) \times \phi(\vec{c})\left[1+\sum_{n=1}^{\infty} a_{n} L_{n}\left(c^{2}\right)\right] \\
& =\frac{d}{2}\left(1-a_{1}\right) .
\end{aligned}
$$

Here we leave out the superscript $\alpha=d / 2-1$ in the notation of $L_{n}^{\alpha}(x)$ for shorter notation as we do below.

Now we can start to calculate the first coefficient $a_{1}$ of the normalized velocity distribution function $F(\vec{c})$ defined by equation (A.18). The density of the kinetic energy is

$$
\int \mathrm{d} \vec{v} \frac{m}{2} v^{2} f(\vec{v}, t)=\frac{m}{2} v_{0}^{2} n \int \mathrm{d} \vec{c} c^{2} F(\vec{c})=\left\langle c^{2}\right\rangle \frac{m}{2} v_{0}^{2} n .
$$

From the definition of temperature $T_{g}(\mathrm{~A} .17)$ and thermal velocity $v_{0}(\mathrm{~A} .16)$ we get

$$
\int \mathrm{d} \vec{v} \frac{m}{2} v^{2} f(\vec{v}, t)=\frac{d}{2} n T_{g}=\frac{d}{2} n \frac{m}{2} v_{0}^{2}
$$


and therefore can identify the second moment of the velocity distribution to be

$$
\left\langle c^{2}\right\rangle=\frac{d}{2} \text {. }
$$

We set Eq. (A.30) equal to Eq. (A.27) and find

$$
a_{1}=0 .
$$

We restrict the approximation of the scaled velocity distribution in this chapter to the first non-trivial coefficient of the Laguerre expansion, which is $a_{2}$, so that the scaled velocity distribution reduces to

$$
F(\vec{c})=\phi(\vec{c})\left[1+a_{2} L_{2}\left(c^{2}\right)\right] .
$$

The rest of this chapter is dedicated calculating $a_{2}$ using the Boltzmann equation as it was done in Goldshtein and Shapiro (1995), van Noije and Ernst (1998) and van Noije (1999) for a dry granular gas.

\section{A.2.3. Moments of the Boltzmann equation}

We calculate the velocity distribution in a stationary state and therefore add some driving, to inject energy, to the Boltzmann equation. We use the simplest form of random forcing which is a stochastic thermostat using white noise. This means that the different stochastic forces $\xi$ are temporal and spatial uncorrelated and we write

$$
\left\langle\xi_{i}^{\alpha}(t) \xi_{j}^{\beta}\left(t^{\prime}\right)\right\rangle=2 \gamma \delta_{\alpha \beta} \delta_{i j} \delta\left(t-t^{\prime}\right),
$$

where angular brackets denote the average over the noise source, $\gamma$ is the strength of the driving, $i, j$ are different particles, $\alpha, \beta$ are cartesian components of the $d$ dimensional space, $t, t^{\prime}$ are two points in time and $\delta$ with an index is the Kronecker delta whereas the function $\delta\left(t-t^{\prime}\right)$ is the Dirac delta function.

The Boltzmann equation changes in the driven stationary state with rescaled quantities to

$$
0=I(F, F)+\underbrace{\frac{\gamma l}{v_{0}^{3}} \frac{\partial^{2}}{\partial \vec{c}_{1}^{2}} F\left(\vec{c}_{1}\right)}_{\text {driving }}
$$

and the collision integral $I(F, F)$ with rescaled quantities reads

$$
I(F, F) \equiv \sigma^{2} \int \mathrm{d} \vec{c}_{2} \int \mathrm{d} \vec{\sigma} \Theta\left(-\vec{c}_{12} \times \vec{\sigma}\right)\left|\vec{c}_{12} \times \vec{\sigma}\right|\left(\chi F\left(\vec{c}_{1}^{* *}, t\right) F\left(\vec{c}_{2}^{* *}, t\right)-F\left(\vec{c}_{1}, t\right) F\left(\vec{c}_{2}, t\right)\right) .
$$

We take the $n^{\text {th }}$ moment of the Boltzmann equation:

$$
\frac{\gamma l}{v_{0}^{3}} \int \mathrm{d} \vec{c}_{1} c_{1}^{n} \frac{\partial^{2}}{\partial \vec{c}_{1}^{2}} F\left(\vec{c}_{1}\right)=-\int \mathrm{d} \vec{c}_{1} c_{1}^{n} I(F, F) .
$$


After integration by parts we obtain for the left hand side of equation (A.36):

$$
\frac{\gamma l}{v_{0}^{3}} \int \mathrm{d} \vec{c}_{1} c_{1}^{n} \frac{\partial^{2}}{\partial \vec{c}_{1}^{2}} F\left(\vec{c}_{1}\right)=\frac{\gamma l}{v_{0}^{3}} n(d+n-2)\left\langle c^{n-2}\right\rangle .
$$

We define the right hand side of equation (A.36), which is the $n^{\text {th }}$ moment of the collision integral, as $v_{n}$ :

$$
v_{n} \equiv-\int \mathrm{d} \vec{c}_{1} c_{1}^{n} I(F, F) .
$$

Using the useful relation (A.14) we can write

$$
v_{n}=-\frac{\sigma^{2}}{2} \int \mathrm{d} \vec{c}_{1} \mathrm{~d} \vec{c}_{2} \mathrm{~d} \vec{\sigma} \Theta\left(-\vec{c}_{12} \vec{\sigma}\right)\left|\vec{c}_{12} \vec{\sigma}\right| F\left(\vec{c}_{1}, t\right) F\left(\vec{c}_{2}, t\right) \times \frac{1}{2}\left[\vec{c}_{1}^{* n}+\vec{c}_{2}^{* n}-\vec{c}_{1}^{n}-\vec{c}_{2}^{n}\right] .
$$

To evaluate this expression, we need the rescaled collision rules:

$$
\begin{aligned}
& \vec{c}_{1}^{*}=\vec{c}_{1}-\frac{\vec{\sigma}}{2}\left(\vec{c}_{12} \vec{\sigma}\right)-\frac{\vec{\sigma}}{2} \sqrt{\left(\vec{c}_{12} \vec{\sigma}\right)^{2}-\varepsilon_{\mathrm{cb}}} \\
& \vec{c}_{2}^{*}=\vec{c}_{2}+\frac{\vec{\sigma}}{2}\left(\vec{c}_{12} \vec{\sigma}\right)+\frac{\vec{\sigma}}{2} \sqrt{\left(\vec{c}_{12} \vec{\sigma}\right)^{2}-\varepsilon_{\mathrm{cb}}}
\end{aligned}
$$

where $\vec{c}_{1}$ and $c_{1}^{*}$ is the velocity before and after the collision respectively and $\varepsilon_{\mathrm{cb}}=$ $2 \times E_{\mathrm{cb}} / v_{0}^{2}$ is the dimensionless bridge energy. For the product $F\left(\vec{c}_{1}, t\right) F\left(\vec{c}_{2}, t\right)$ we only keep terms which are of linear order in $a_{2}$ :

$$
F\left(\vec{c}_{1}, t\right) F\left(\vec{c}_{2}, t\right)=\phi\left(\vec{c}_{1}\right) \phi\left(\vec{c}_{2}\right)\left(1+a_{2}\left[L_{2}\left(c_{1}^{2}\right)+L_{2}\left(c_{2}^{2}\right)\right]\right) .
$$

For the second and fourth moment of the Boltzmann equation we find

$$
\begin{aligned}
\frac{\gamma l}{v_{0}^{3}} 2 d\left\langle c^{0}\right\rangle & =v_{2} \\
\frac{\gamma l}{v_{0}^{3}} 4(d+2)\left\langle c^{2}\right\rangle & =v_{4} .
\end{aligned}
$$

To find an expression for $a_{2}$ we have to solve

$$
\frac{v_{4}}{v_{2}}=d+2 \text {. }
$$

\section{A.2.3.1. Second moment}

To calculate the moment $v_{2}$ of the collision integral, we introduce the center of mass velocity $\vec{V}$ and the relative velocity $\vec{u}$

$$
\begin{aligned}
& \vec{c}_{1}=\vec{V}+\frac{\vec{u}}{2} \\
& \vec{c}_{2}=\vec{V}-\frac{\vec{u}}{2} .
\end{aligned}
$$


We divide $\vec{V}$ and $\vec{u}$ into parts which are parallel and perpendicular to the collision vector $\vec{\sigma}$, respectively:

$$
\begin{aligned}
& \vec{u}_{\perp} \equiv \vec{u}-(\vec{u} \vec{\sigma}) \vec{\sigma}, \quad u_{\sigma}=\vec{u} \vec{\sigma} \\
& \vec{V}_{\perp} \equiv \vec{V}-(\vec{V} \vec{\sigma}) \vec{\sigma}, \quad V_{\sigma}=\vec{V} \vec{\sigma} .
\end{aligned}
$$

From the rescaled collision rules (A.40) and (A.41) we find the collision rules in these new variables:

$$
\begin{aligned}
& \vec{c}_{1}^{*}=\vec{V}_{\perp}+\frac{\vec{u}_{\perp}}{2}+\left(V_{\sigma}-\frac{\sqrt{u_{\sigma}^{2}-\varepsilon_{\mathrm{cb}}}}{2}\right) \vec{\sigma} \\
& \vec{c}_{2}^{*}=\vec{V}_{\perp}-\frac{\vec{u}_{\perp}}{2}+\left(V_{\sigma}+\frac{\sqrt{u_{\sigma}^{2}-\varepsilon_{\mathrm{cb}}}}{2}\right) \vec{\sigma} .
\end{aligned}
$$

We can now calculate

$$
\frac{1}{2}\left(\vec{c}_{1}^{* 2}+\vec{c}_{2}^{* 2}-\vec{c}_{1}^{2}-\vec{c}_{2}^{2}\right)=-\frac{\varepsilon_{\mathrm{cb}}}{4}
$$

and

$$
L_{2}\left(c_{1}^{2}\right)+L_{2}\left(c_{2}^{2}\right)=\left(\vec{V}^{2}+\frac{\vec{u}^{2}}{4}\right)^{2}+(\vec{V} \vec{u})^{2}-(d+2)\left(\vec{V}^{2}+\frac{\vec{u}^{2}}{4}\right)+\frac{d(d+2)}{4} .
$$

If we substitute equation (A.53) in (A.42) and the result (with the respective change of variables) together with (A.52) in equation(A.39), we obtain

$$
\begin{aligned}
v_{2} & =\frac{\varepsilon_{\mathrm{cb}}}{4} \Omega_{d} \int_{u_{\sigma} \geqslant \sqrt{\varepsilon_{\mathrm{cb}}}} \mathrm{d} \vec{V} \mathrm{~d} \vec{u} \frac{\mathrm{e}^{-2 \vec{V}^{2}-\frac{\vec{u}^{2}}{2}}}{\pi^{d}} u_{\sigma} \\
& \times\left[1+a_{2}\left(\left(\vec{V}^{2}+\frac{\vec{u}^{2}}{4}\right)^{2}+(\vec{V} \vec{u})^{2}-(d+2)\left(\vec{V}^{2}+\frac{\vec{u}^{2}}{4}\right)+\frac{d(d+2)}{4}\right)\right],
\end{aligned}
$$

where $\Omega_{d}$ is the $d$-dimensional solid angle. This integral can be evaluated using the variable substitution of $(\mathrm{A} .48)$ and $(\mathrm{A} .49)$ and we obtain

$$
v_{2}=\frac{e^{-\varepsilon_{\mathrm{cb}} / 2} \pi^{-\frac{1}{2}+\frac{d}{2}} \varepsilon_{\mathrm{cb}}\left(16+a_{2}\left(\left(\varepsilon_{\mathrm{cb}}-2\right)-1 \varepsilon_{\mathrm{cb}}\right)\right)}{32 \sqrt{2} \times \Gamma\left(\frac{d}{2}\right)} .
$$

\section{A.2.3.2. Fourth moment}

To evaluate the fourth moment of the collision integral, we first calculate

$$
\begin{aligned}
\frac{1}{2}\left(\vec{c}_{1}^{* 4}+\vec{c}_{2}^{* 4}-\vec{c}_{1}^{4}-\vec{c}_{2}^{4}\right)= & -\frac{\varepsilon_{\mathrm{cb}}}{2}\left(3 V_{\sigma}^{2}+\frac{1}{4} u_{\sigma}^{2}-\frac{\varepsilon_{\mathrm{cb}}}{8}+\vec{V}_{\perp}^{2}+\frac{\vec{u}_{\perp}^{2}}{4}\right) \\
& -2 V_{\sigma}\left(u_{\sigma}+\sqrt{u_{\sigma}^{2}-\varepsilon_{\mathrm{cb}}}\right) \vec{V}_{\perp} \vec{u}_{\perp}
\end{aligned}
$$


which follows straightforward calculation. Analogous to the calculation of the second moment, we find for the fourth moment

$$
\begin{aligned}
v_{4}= & -\Omega_{d} \int_{u_{\sigma} \geqslant \sqrt{\varepsilon_{\mathrm{cb}}}} \mathrm{d} \vec{V} \mathrm{~d} \vec{u} \frac{\mathrm{e}^{-2 \vec{V}^{2}-\frac{\vec{u}^{2}}{2}}}{\pi^{d}} u_{\sigma} \\
& \times\left[1+a_{2}\left(\left(\vec{V}^{2}+\frac{\vec{u}^{2}}{4}\right)^{2}+(\vec{V} \vec{u})^{2}-(d+2)\left(\vec{V}^{2}+\frac{\vec{u}^{2}}{4}\right)+\frac{d(d+2)}{4}\right)\right] \\
\times & {\left[-\frac{\varepsilon_{\mathrm{cb}}}{2}\left(3 V_{\sigma}^{2}+\frac{1}{4} u_{\sigma}^{2}-\frac{\varepsilon_{\mathrm{cb}}}{8}+\vec{V}_{\perp}^{2}+\frac{\vec{u}_{\perp}^{2}}{4}\right)-2 V_{\sigma}\left(u_{\sigma}+\sqrt{u_{\sigma}^{2}-\varepsilon_{\mathrm{cb}}}\right) \vec{V}_{\perp} \vec{u}_{\perp}\right], }
\end{aligned}
$$

which we evaluate again in the same manner. However, because the solution is a bit more lengthy than for $v_{2}$ we do not give it explicitly here.

\section{A.2.4. Result}

We now have the explicit solutions for $v_{2}$ and $v_{4}$. Therefore, equation (A.45) is solvable with respect to $a_{2}$. The full solution reads

$$
a_{2}=-\frac{16\left(\varepsilon_{\mathrm{cb}}-2\right) \varepsilon_{\mathrm{cb}}}{-128+16 d\left(8+\varepsilon_{\mathrm{cb}}\left(5+\varepsilon_{\mathrm{cb}}\right)\right)+\varepsilon_{\mathrm{cb}}\left(10+\varepsilon_{\mathrm{cb}}\left(75+\left(\varepsilon_{\mathrm{cb}}-4\right) \varepsilon_{\mathrm{cb}}\right)\right)+Z_{s}}
$$

with $Z_{s}=32(d-1) \varepsilon_{\mathrm{cb}} \exp \left(\varepsilon_{\mathrm{cb}} / 4\right) K_{1}\left(\frac{\varepsilon_{\mathrm{cb}}}{4}\right)$ where $K_{1}(x)$ is the first order modified Bessel function of the second kind.

Expanding equation (A.59) in powers of $\varepsilon_{\mathrm{cb}}$ for small values around zero we obtain:

$$
a_{2}=\frac{1}{8(d-1)} \varepsilon_{\mathrm{cb}}-\frac{15(8 d-5)}{1024(d-1)^{2}} \varepsilon_{\mathrm{cb}}^{2}+O\left(\varepsilon_{\mathrm{cb}}\right)^{3} \text {. }
$$

This result can be used to calculate the Fourier law of heat conduction similar as it was done for dry granulates (Deltour and Barrat, 1997; Soto et al., 1999). 
A. Velocity distribution of a wet granular gas 


\section{B. Sinusoidal shaking in event-driven simulations}

Event driven algorithms are a powerful and efficient simulation method that can be used to numerically study the behavior of particulate systems, such as granular materials or powders. Its superior efficiency originates from only considering times, where physically relevant events occur, as long as these times can be calculated analytically. This, however, is only possible for pairs of trajectories whose relative distance can be expressed as a polynomial of order less than or equal to four. For more complex trajectories, like a sinusoidal motion of a wall, the collision times have to be calculated numerically, which dramatically reduces the efficiency. In this chapter we present a tabulation method implemented in an event-driven algorithm, which is able to efficiently handle the one-dimensional motion of a periodically moving wall. While demonstrating this for a sinusoidally moving wall, we simplify the comparability of simulations with (usually sinusoidally driven) experiments. Because granular materials or powders are systems which are easily accessible through both experiments and simulations, our work enhances the growing field of statistical physics far from equilibrium.

\section{B.1. Introduction}

Whether in nature as sand or coarse gravel, or in industrial processes and products like mining, food processing, or the pharmaceutical industry, granular materials or powders are omnipresent. Because these systems of particles are out of equilibrium, the concepts and tools known from classical thermodynamics, like phase diagrams or thermodynamic potentials, unfortunately do not universally apply in these cases. Due to the multitude of individual particles, it is a challenge to predict the behavior of such collective systems, and, for example, phase diagrams have to be extracted laboriously for any non-equilibrium system. Thus, it is advantageous to develop simulation techniques which allow a fast and efficient exploration of the desired bulk behavior.

A suitable and efficient ${ }^{1}$ algorithm is the event-driven molecular dynamics (EDMD)

1. with respect to the computing time per physical time 
(Poeschel and Schwager, 2004) simulation. However, as pointed out in Miller and Luding (2004), it has never become as popular as the less efficient time-driven molecular dynamics (TDMD) (Poeschel and Schwager, 2004) simulation method. This seems to be due to an often higher implementation effort combined with less flexibility in EDMD. However, interesting implementations for EDMD were published in recent years, which extend the range of application of this method. For example, there are algorithms for parallelization (Miller and Luding, 2004), the use of non-spherical particles (Donev et al., 2005a,b), a priority queue with complexity $\mathrm{O}$ (1) (Paul, 2007), the collision detection of moving spheres with unknown trajectories (Kim et al., 2005), and the use in a ball mill simulation (Reichardt and Wiechert, 2007). EDMD simulations are not exclusively used in the field of physics but are also investigated in theoretical computer science in the context of Kinetic Data Structures (Mehta and Sahni, 2004).

In this chapter we discuss a tabulation scheme to efficiently simulate moving walls of infinite mass, which are assumed to be flat. Its trajectories have to be mathematically smooth, periodic and finite. The motion of the wall itself is one-dimensional, but can be coupled to a system of any dimension. We demonstrate our method with a sinusoidally moving wall confining a system with circular (two-dimensional) or spherical (three-dimensional) particles. Sinusoidally moving walls in EDMD simulations were used already more than a decade ago (Luding et al., 1994a,b), but are still of interest (Carrillo et al., 2008). Due to the high implementation effort and the loss of computational efficiency approximations by polynomials of the order two, or even less, are used whenever possible (see e. g. McNamara and Luding, 1998). Sometimes even the much slower TDMD simulation method is still used (Xu and O'Hern, 2005).

We increase the computational efficiency of simulating e.g. a sinusoidally moving wall in EDMD simulations to strengthen its usage even in cases where other methods (approximation with polynomials or TDMD simulations) were used until present. Yet, the shape of the driving function becomes important at the point where one wants to compare simulations with experimental results: experiments on wet granulates (Scheel et al., 2004) have shown that the second derivative of the driving function is significant for the nonlinear response of the sample.

\section{B.2. The mathematical task}

The basic idea of EDMD is first to determine the time where the next event occurs, where events are particle-particle collisions or collisions with a wall. The positions of the particles involved in these events are then easily computed in a second step of the evolution along their trajectories for the previously calculated time period. The velocities before the collision are calculated the same way and after applying the collision laws in the final step we obtain the final velocities after the collision. These three steps are then repeated. 


\section{B.2.1. Calculation of the collision time}

\section{B.2.1.1. Particle-particle collision}

To find the collision time $\Delta t$ of two particles $i$ and $j$ we have to solve the equation

$$
\left|\vec{x}_{i}(\Delta t)-\vec{x}_{j}(\Delta t)\right|=R_{i}+R_{j}
$$

with respect to time $\Delta t$, in which $\vec{x}_{i}(\Delta t), \vec{x}_{j}(\Delta t)$ are the trajectories and $R_{i}, R_{j}$ the radii of the particles.

As an example, we take particles moving in a homogeneous gravitational field with gravity $g$ and trajectory

$$
\vec{x}_{i}(\Delta t)=-\frac{1}{2} \vec{g} \Delta t^{2}+\vec{v}_{i} \Delta t+\vec{s}_{i}
$$

where $t$ is the common time basis and $\vec{v}_{i}$ and $\vec{s}_{i}$ is the initial particle velocity and position respectively. Inserting this equation for the two particle trajectories $\vec{x}_{i}(\Delta t)$ and $\vec{x}_{j}(\Delta t)$ in Eq. (B.I) leads to the following quadratic equation in time $\Delta t$ :

$$
\left(\vec{v}_{i}-\vec{v}_{j}\right)^{2} \times \Delta t^{2}+2\left(\vec{v}_{i}-\vec{v}_{j}\right)\left(\vec{s}_{i}-\vec{s}_{j}\right) \times \Delta t+\left(\vec{s}_{i}-\vec{s}_{j}\right)^{2}-\left(R_{i}+R_{j}\right)^{2}=0,
$$

which can easily be solved and provides the desired collision time. The question if there is a collision at all is equivalent to asking if this equation has a real solution while the particles are approaching each other (Brilliantov and Pöschel, 2004).

\section{B.2.1.2. Particle-wall collision}

As stated in the introduction, we focus on components of trajectories with an arbitrary functional form. As an example we choose a wall oscillating around a mean value $\vec{x}_{W_{0}}$ with trajectory $\vec{x}_{W}(\Delta t)=\vec{A} \sin (\omega \Delta t)+\vec{x}_{W_{0}}$, where $\vec{A}$ is the shaking amplitude and $\omega=2 \pi / T_{\text {driv }}$ the angular frequency with period $T_{\text {driv }}$. To calculate the collision time of a particle with the wall, we have to solve

$$
\begin{aligned}
\left|\vec{x}_{i}(\Delta t)-\vec{x}_{W}(\Delta t)\right| & =R_{i} \\
\left|-\frac{1}{2} \vec{g} \Delta t^{2}+\vec{v}_{i} \Delta t+\vec{s}_{i}-\left(\vec{A} \sin (\omega \Delta t)+\vec{x}_{W_{0}}\right)\right| & =R_{i}
\end{aligned}
$$

with respect to time $\Delta t$. This is not possible analytically, and results in a lengthy expression involving the fourth power of $\Delta t$. However, it is possible to simplify the equation without loss of generality by simply choosing a convenient coordinate system: First we choose one axis of the coordinate system to point in the same direction as the amplitude vector of the wall motion. Therefore, the problem reduces to a one-dimensional problem, where only the dimension perpendicular to the wall is considered. Secondly, we place the origin of our coordinate system to the mean position $\vec{x}_{W_{0}}$ of the wall and therefore eliminate this constant. Last but not least, we 
define the axis to be positive on the side of the wall where the respective particle is located. Together with the definition in Eq. (B.4) the absolute value is then redundant. The problem is reduced to the following one-dimensional equation:

$$
\begin{aligned}
s_{\text {rel }}(\Delta t) & =x_{i}(\Delta t)-x_{W}(\Delta t) \\
& =\left(-\frac{1}{2} g \Delta t^{2}+v_{i} \Delta t+s_{i}-R_{i}\right)-(A \sin (\omega \Delta t))=0,
\end{aligned}
$$

where $s_{\text {rel }}(\Delta t)$ is the relative distance between the particle surface $x_{i}(\Delta t)$ and the wall which is located at $x_{W}(\Delta t)$. This expression is of course still not analytically solvable with respect to time $\Delta t$, but it is more convenient for the task of finding its roots.

\section{B.2.2. Two reasonable choices for the frame of reference}

There are two reasonable choices for the frame of reference in which the simulations can be performed: Either being outside in the laboratory or being inside and moving with the oscillating wall. So far, we have implicitly performed the calculations in the laboratory frame of reference and found Eq.(B.7) as the resulting equation that has to be solved. Actually, Eq.(B.7) does not change when using the frame of reference of the moving wall, only the contributions arise from the particle position $x_{i}(\Delta t)=-\frac{1}{2} g \Delta t^{2}+v_{i} \Delta t+s_{i}-R_{i}-A \sin (\omega \Delta t)$ while $x_{W}(\Delta t)=0$. Aside from the advantage of a stationary wall, the main disadvantage of the moving wall as the frame of reference is due to performance issues. The computation of the particle position $x_{i}(\Delta t)$ involves the evaluation of the sine function whenever propagating a particle along its trajectory, which is necessary for every single event. Due to the comparatively high computational effort of evaluating a sine or cosine function, this choice significantly reduces the computational efficiency. This is the main reason why we have chosen the laboratory as the frame of reference in our simulations.

\section{B.3. A first algorithm}

After finding the roots we will introduce the minimal ingredients to successfully perform the EDMD simulation, and we will recognize at the end of this section that we lose a lot of the EDMD efficiency, which we will subsequently enhance in Sec. B.4.

\section{B.3.1. Back to the roots}

Numerically finding the roots of a function $f(t)$ is a standard problem in numerical mathematics and can be found in every textbook on the subject, e.g. Press et al. (1992). "Perhaps the most celebrated of all one-dimensional root-finding routines is Newton's method, also called Newton-Raphson method" (Press et al., 1992).

Newton's method extrapolates the functional value $f\left(t_{i}\right)$ of an initial guess for the value of the root $t_{i}$ along its tangent line until it crosses zero. This crossing point $t_{i+1}$ 
is the initial value for the next iteration step. The Newton-Raphson formula

$$
t_{i+1}=t_{i}-\frac{f\left(t_{i}\right)}{f^{\prime}\left(t_{i}\right)}
$$

therefore, involves both the function and its derivative, which have to be known for any time $t_{i}$. The derivative obviously has to be continuous and nonzero around the true value of the root. The method derives mathematically from the Taylor series expansion taking only linear terms into account, and thus is a good approximation in the region around the true value of the root. However, outside that region, where higher order terms dominate the result, this method leads to poor results. Another problem of the method occurs when an initial guess is close to a local extremum, where the derivative is close to zero and drives the value in the next iteration step far away from the true one.

Nevertheless, this method is often used because of its good convergence behavior close to the true value of the root, where every iteration step doubles the number of correct digits. For numerical implementations a recipe exists (Press et al., 1992) for combining Newton's method with a bisection method to overcome its negative features, but still profit (when possible) from its good convergence behavior. Therefore, at each iteration step the values for $t_{i}$ are bounded to a certain interval around the root. If Newton's method drives the value $t_{i}$ out of this range, the bisection method continues the search. In order for this combination to work successfully, one and only one root can lie within this interval.

\section{B.3.2. Search for unique bracketing bounds}

The next step in successfully solving Eq. (B.7) with this safe root-finding method is to find the bracketing bounds which uniquely clasp around the wanted root. Eq. (B.7) is infinitely times differentiable and therefore has an infinite number of roots, however, it is obvious that the next collision corresponds to the root that is closest in time, which is the one with the smallest value of $\Delta t$. Unfortunately, two roots of (B.7) can come arbitrarily close to what physically corresponds to 'grazing shots', which hit the wall nearly tangentially. Because we have seen that one and only one root must lie within the interval, we have to search for the suitable bounds for every root individually and cannot use e.g. a default initialization, which works in any (or at least most) cases. In fact, we additionally have to define a minimum interval length $t_{\min }$ for which we are able to distinguish two individual roots. This also defines the limit of resolving 'grazing shots'. Because the transfer of momentum by 'grazing shots' is extremely small, it is physically irrelevant to which extent they can be resolved. The challenge is more a computational one, because one has to be able to deal with particles overlapping with the wall. This is shown in more detail in subsection B.5.6. The choice of $t_{\min }$ is therefore determined by the maximum overlap one is able to handle. 
Finding the interval which contains the root means testing at $\Delta t=n \times t_{\min }$, where $n \in\{1,2, \ldots\}$ for a change of sign in the function $s_{\text {rel }}(\Delta t)$ in (B.7). The interval containing the root is $\left[(n-1) t_{\min }, n t_{\min }\right]$ if a change of sign was detected at $n$. This searching procedure is sketched schematically in Fig. B.I, where a shows a successful run, whereas in part $\mathbf{b}$ for the same function no pair of brackets is found because the time interval $t_{\min }$ was too big. This search is of linear complexity $\mathrm{O}(n)$ in the number of intervals, or equivalently of complexity $\mathrm{O}\left(1 / t_{\min }\right)$ in the minimum time resolution. The complexity unfortunately cannot be reduced with the use of other search algorithms.

a

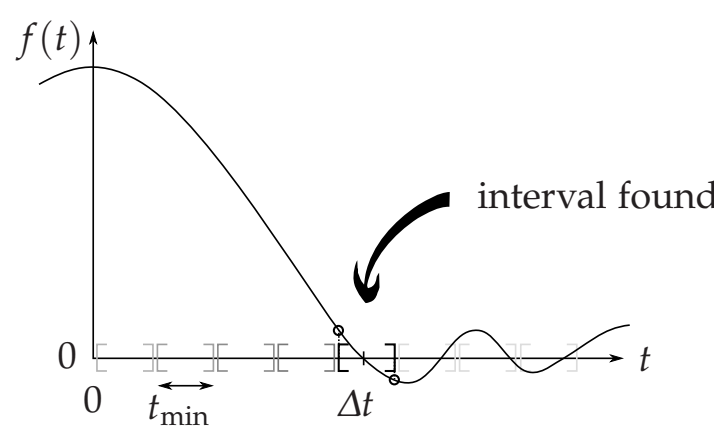

b

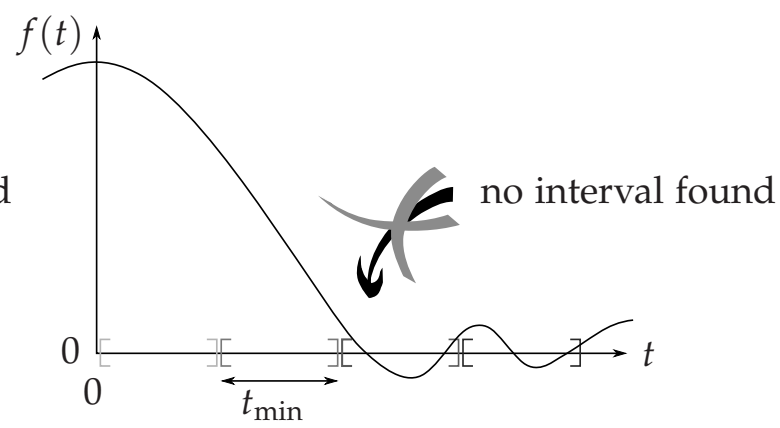

Fig. B.1 Schematic sketch of the search for bracketing bounds around the smallest root of a function $f(t)$. The length of one interval is $t_{\min }$ which is twice as large in $\mathbf{b}$ as in $\mathbf{a}$. The sequence of intervals are tested to see whether $f(t)$ changes sign within the range, and are denoted by different gray levels. In $\mathbf{a}$ the search was successful whereas in $\mathbf{b}$ the interval length was too big and no brackets could be found.

With the knowledge of the appropriate interval we are able to successfully find the accurate solution of (B.7). However, a drawback is that for trajectories, where the particle does not hit the wall, $n$ is unbounded and the algorithm does not terminate. It is simply not possible to explicitly see in the function if there is at least one positive real solution, which would correspond to a wall-collision in the future.

\section{B.3.3. Assure termination of the algorithm}

To assure that the algorithm terminates, even when there is no collision with the wall, we have to find a criterion that defines a limit for the number of intervals $n$ which have to be tested for a change of sign in the relative distance (B.7). As we will see, we are able to define a limit by requiring boundedness and periodicity of the components' trajectory.

\section{B.3.3.1. The starting point}

As mentioned earlier, we are searching the bracketing bounds around the root with the smallest positive value. As a starting point for the search we use the present time 
of the particle, which defines the origin of the time axis. In a first step we are now able to optimize the time where we have to start the search if the range of action of the wall is bounded, meaning that the function of the wall trajectory is finite for all times.

In Fig. B.2 we see a space-time plot of the sinusoidal wall motion and particle (1), which collides on a parabolic trajectory with the wall, whereas particle (2) does not. In particular, note that the sign of the gravitational force is different for these two trajectories. The gray area denotes the region behind the wall that is unreachable for particles. The bold line $I$ denotes the maximum amplitude and is located at $x_{I}=A$, the maximal range of action of the wall.

A necessary condition for a particle to collide with the wall is $x \leqslant x_{I}$, so it has to be below line $I$, in order to be able to hit the wall. It is straightforward to calculate the time $t_{E}$ when the particle trajectory crosses line $I$, and we start searching the bracketing bounds not before this condition is fulfilled. This is only relevant for particles being located above line $I$ at their present time. Therefore, it is possible to reduce the number of intervals to find the bracketing bounds from $n_{0}$ to $n_{1}$, where $n_{0}$ is the original number of intervals from the present time up to the collision whereas $n_{1}$ is the reduced number counted from the crossing of line $I$ up to the collision (cf. Fig. B.2). For particles initially located below line $I$, nothing changes and we perform the search right from their present time.

As the particle trajectory (2) in Fig. B.2 clearly illustrates, there are still infinitely many intervals to check if there is no collision with the wall. However, we can demand our driving to be periodic with the periodic time $T_{\text {driv }}$. Once a particle is then located below line $I$ it has to collide with the wall until the end of the current period, which is when the wall reaches its maximum amplitude again. Now we can stop searching at the end of the current period, even if there will be no collision with the wall. The number of intervals which we have to test is $n=\left(T_{\text {driv }}-t_{E}\right) / t_{\min }$, where $t_{E}$ is the entry time into the range of action of the wall relative to the respective period. In the worst case this leads to a maximum number of

$$
n_{\max }=\frac{T_{\text {driv }}}{t_{\min }}
$$

intervals which have to be tested. For any fixed $t_{\min }$ this is a finite value, as required.

\section{B.3.3.2. The exception}

There is one remaining exception for the limit described above. When the particle is starting below $I$, e.g. directly after a wall collision, and there is no collision within the respective period, there will be nevertheless a wall collision in the case when gravity points in the direction of the wall. This is sketched in Fig. B.3.

Fortunately it is possible to solve this issue with the method described above. After realizing that there is no collision within the respective period, we calculate 


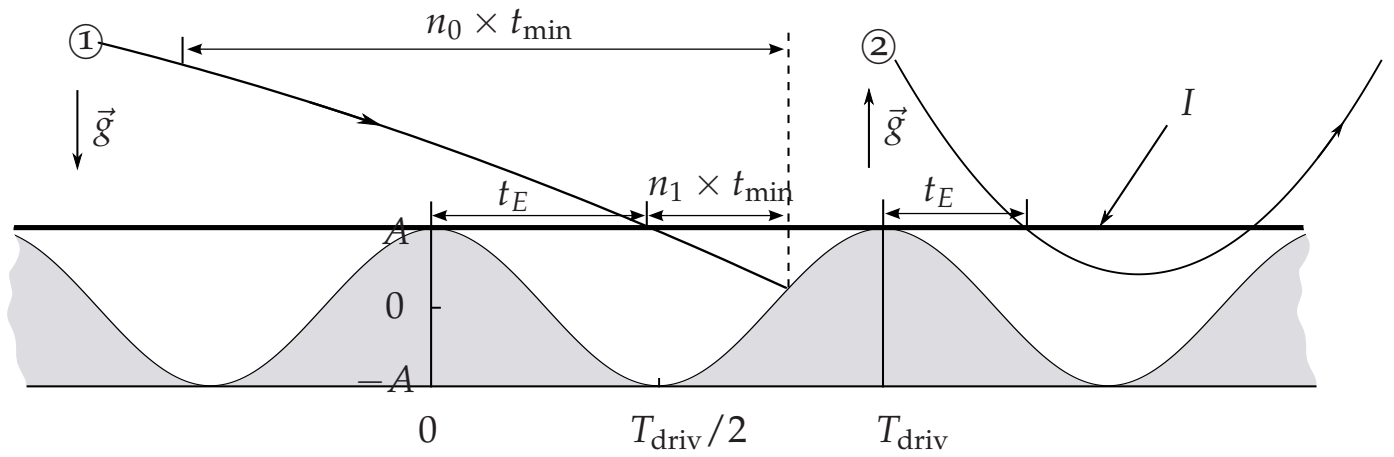

Fig. B.2 The bold line $I$ denotes the maximum range of action of the sinusoidally moving wall in this space-time plot. The gray area denotes the region behind the wall which is unreachable for the particles. Once a particle trajectory crosses this line from above it has to collide with the wall within the respective period like particle (1) or it will never collide, like particle (2). This limits the number of intervals for searching the bracketing bounds which is necessary for the algorithm to terminate in any case. Note the different sign in the gravity $\vec{g}$ of the two particle trajectories in this sketch. This additionally improves the efficiency due to reduction of $n_{0}$ to $n_{1}$ relevant intervals, because the search only starts when entering the range of action of the wall at time $t_{E}$ measured relative to the respective period.

the time $t_{1}$ when the particle reenters the range of action of the wall and use this time as a final starting point for searching the bracketing bounds as described above. The number of intervals that are tested in this case is $n=n_{1}+n_{2}$, where $n_{1}$ is the number of intervals until the end of the first period and $n_{2}$ the remaining intervals from reentry until collision. This leads to the worst case for the number of intervals

$$
n_{\max }=2 \frac{T_{\text {driv }}}{t_{\min }}
$$

which is twice as large as in the usual case of Eq. (B.9) but still denotes an upper limit.

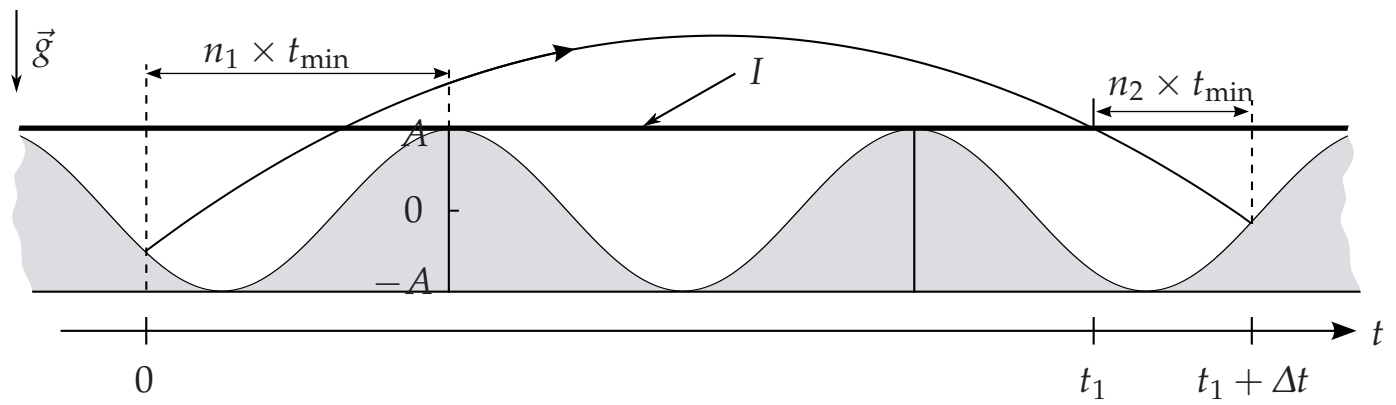

Fig. B.3 When a particle is located below line $I$, e.g. after a preceding wall collision, but there is no wall collision within the respective period it is, however, possible that there is a wall collision at a later time if gravity $\vec{g}$ points in the direction of the wall. We then use the time $t_{1}$ when the particle reenters the range of action of the wall as a starting point for the final search of the bracketing bounds. The number of intervals which are tested is then $n_{1}+n_{2}$. 
Although the complexity of the search algorithm to find the bracketing bounds was reduced and limited, it is still a search which has a linear complexity. This method therefore is very expensive and we are losing a lot of the efficiency, which is the main advantage of EDMD.

\section{B.4. Improving the efficiency with look up tables}

The main purpose of this task is to speed up the simple algorithm that was discussed in the previous section. Because the search of unique bracketing bounds is very expensive, this is the critical point. We have already seen that finding one bracketing bound cannot be further accelerated, so we can only reduce the number of calculations by storing each result in a look up table. This, of course, only saves computation time if we are able to reuse the stored values as often as possible. As we shall explore in detail, this tabulation is not achieved in a trivial manner.

\section{B.4.1. What to store in the table}

We spend the most computational effort in finding the bracketing bounds for the root, so this is a candidate to store in the table. However, we can go one step further. Thus, instead of the brackets, we store the solution of Eq.(B.7) in the table, which physically is the time $\Delta t$ when the wall collision takes place.

The solution $\Delta t$ is calculated for a certain point on a trajectory, which naturally is the origin of the coordinate system in which the time $\Delta t$ is measured. However, if it is possible to analytically calculate the time $t_{a}$ from any other point on the trajectory to that origin, it is straightforward to calculate the time when the collision takes place from that point. It simply is $t_{a}+\Delta t$. Therefore, we only have to calculate and store the solution for one point of each trajectory.

To define the points of the origin of $\Delta t$ for different trajectories is reminiscent of finding the starting point for searching the bracketing bounds in Sec.B.3.3.1. Indeed, we are using the line $I$ from Fig.B.2, which was the starting point for finding the bracketing bound, also as the origin for the time $\Delta t$. Due to the periodicity of the wall motion, it is sufficient only to consider trajectories within one period. The time when the collision takes place is then the time $t_{E_{I}}$, respective to the current period when the trajectory crosses the line $I$, plus the value $\Delta t$ for that trajectory out of the table (cf. Fig. B.4). However, not every trajectory intersects with line $I$ and therefore, we introduce a line II as can be seen in Fig. B.4, which is located at $x_{I I}=-A$ and defines the minimum value of the wall motion. It is used in the same way as the line $I$.

The origin of $\Delta t$ is equal to the starting point for searching the bracketing bound. We therefore introduce a line $I I I$, which indicates the beginning of the respective period in which the particle collides with the wall. This is to limit the number of intervals, according to Eq. (B.9) which is equivalent to limit the collision time 
$0 \leqslant \Delta t \leqslant T_{\text {driv }}$.

As can be seen in Fig. B.4 the lines define a frame around one period of the wall motion and we consequently refer to them, not as lines anymore, but as edges $I, I I$ and III. The intersection of the trajectories with these edges defines the base points from which we measure $\Delta t$, which is stored in the table.

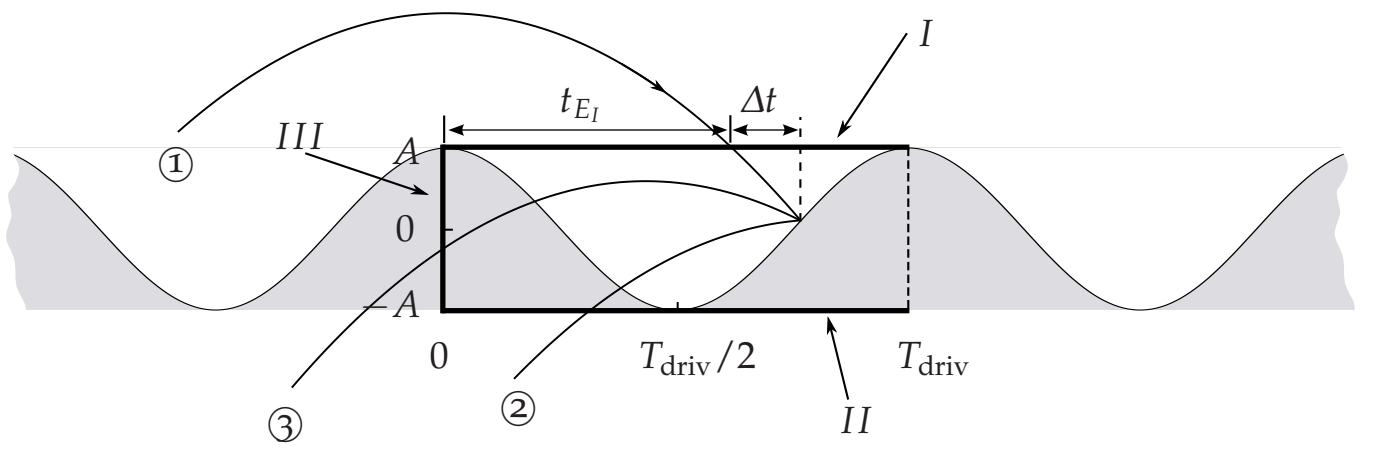

Fig. B.4 A frame with edges $I, I I$ and $I I I$ around one period of the sinusoidal wall motion denotes the points of origin for the time $\Delta t$ up to the next wall collision for the different trajectories. Edge $I$ is located at the maximum $x_{I}=A$ and edge $I I$ at the minimum $x_{I I}=-A$ of the wall motion. Edge III denotes the beginning of the respective period. This ensures that the collision time $\Delta t$ fulfills the condition $0 \leqslant \Delta t \leqslant T_{\text {driv. }}$. This is necessary to define an upper limit for the number of intervals that have to be tested as bracketing bounds.

If there is more than one real solution $\Delta t$ for a given trajectory, we store all solutions $\Delta t$ which lie within the current period. This raises two questions: The first question, which is answered in Sec. B.4.5, is why we need all these solutions and how we are going to use them. The second question, which is answered in the following subsection, is how to use trajectories as parameters for a table. This question arises due to the fact that there are infinitely many different trajectories.

\section{B.4.2. Trajectories as parameters of the table}

To specify a certain trajectory, cf. Eq. (B.2), there are three independent parameters: We have to choose the time basis $t$, the initial particle velocity $v_{i}$ and the initial particle position $s_{i}$.

Because every edge denotes either one spatial or temporal condition, there are only two remaining free parameters to classify all possible trajectories. The first free parameter is the one which completes (together with the condition of the edge) the definition of a space-time point. The second parameter is, in all cases, the particle velocity perpendicular to the wall at that space-time point. This means that for edge $I$, with the spatial localization at $x_{I}=A$, the first parameter is temporal and, more precisely, the time $t_{E_{I}}$ when the trajectory intersects with the edge relative to the respective period. The second parameter is the velocity $v_{E_{I}}$ at that base point. For edge $I I$ it is quite similar. The spatial localization is $x_{I I}=-A$ and we need 
a temporal parameter to complete the definition of a point in space-time, which is again the time $t_{E_{I I}}$ when crossing the edge. The second parameter is again the velocity $v_{E_{I I}}$ at that point. This is shown in Fig. B.5a. The condition for the location of edge III, however, is a temporal one since it is located at $t=0$, which denotes the beginning of the respective period. The first parameter characterizing the base point on the edge is therefore the height $h_{E}$ at which the intersection takes place. And of course, the second parameter for this edge is, as usual, the velocity $v_{E_{I I I}}$ at that space-time point. This is shown in Fig. B.5b.

With the help of these three edges we reduced the initial three-dimensional parameter space, which was necessary to fully classify all possible trajectories to three two-dimensional ones. Therefore, we use a separate two-dimensional table for each edge with the two parameters as described above.

Some trajectories occur in more than one table, so we can limit the edge $I$ to trajectories with $v_{E}<0$ and the edge $I I$ to $v_{E}>0$ in order to reduce redundancy. Nevertheless, some entries occur several times and in these cases we choose the table with the lowest value of $\Delta t$ to minimize numerical errors. This leads to the sequence I, II, III of the edges in our example.

To tabulate the values it is now evident that we have to discretize the parameters of the tables, as for any other standard tabulation. The values $t_{E_{I}}$ and $t_{E_{I I}}$ are finite due to the periodicity of the wall motion, and $h_{E}$ is finite because in Sec.B.3.3.1 we required the range of action of the wall to be finite. Therefore, the discretization of these parameters is straightforward. However, the velocity $v_{E}$ at all edges is unbounded, so it is neither reasonable nor possible to tabulate it directly on a linear scale.

a

(1)

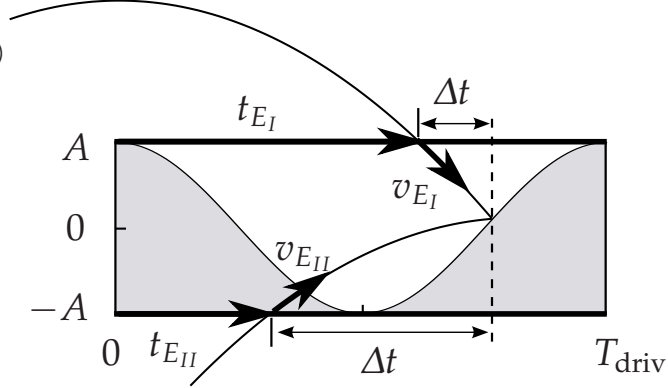

(2) b

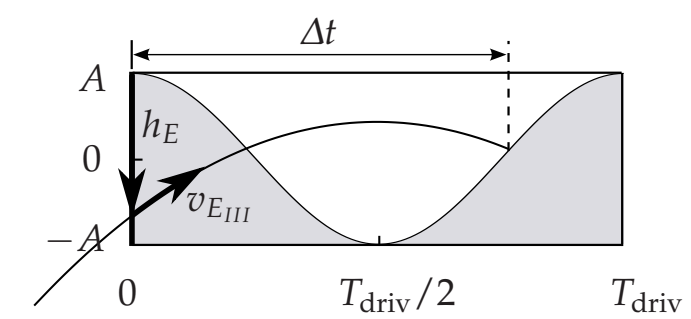

(3)

Fig. B.5 a Trajectory (1) (2)) is characterized by the time $t_{E_{I}}\left(t_{E_{I I}}\right)$ when crossing the edge $I(I I)$ and the velocity $v_{E_{I}}\left(v_{E_{I I}}\right)$ perpendicular to the wall at that point. The time $\Delta t$ until the next wall collision takes place is stored in the look-up table. b Trajectory (3) is characterized by the height $h_{E}$ when crossing edge $I I I$ and the velocity $v_{E_{I I I}}$ at that point and again time $\Delta t$ is stored in the look-up table. 


\section{B.4.3. The mapping of velocities}

The velocities $v_{E}$ for all edges are unbounded, so the standard discretization method of linearly distributing the table entries over the whole range of possible values is certainly not applicable here. Nonetheless, it is possible to define a mapping function $f\left(v_{E}\right)$ for the velocity and tabulate the mapped velocity $f\left(v_{E}\right)$ instead of $v_{E}$. A reasonable choice is to use relation (B.11) or (B.12)

$$
\begin{aligned}
& f\left(v_{E}\right) \propto \arctan \frac{v_{E}}{v_{0}} \\
& f\left(v_{E}\right) \propto \tanh \frac{v_{E}}{v_{0}},
\end{aligned}
$$

where the unbounded velocity $v_{E}$ is mapped to a finite interval. There are also other possibilities such as relation (B.13) or (B.14)

$$
\begin{array}{cc}
f\left(v_{E}\right) \propto & \log \left(v_{E}\right) \\
f\left(v_{E}\right) \propto & \frac{1}{v_{E}},
\end{array}
$$

which is interesting because there is a higher resolution in the physically relevant range of small velocities. However, in these cases the velocity still has to be bounded with a minimum and a maximum cut-off velocity. The first derivatives of the mapping functions account for the density of table entries at the respective velocity and therefore it is favorable to adjust the mapping function according to the respective problem. In the present work we are using the logarithmic scaling of Eq. (B.13).

\section{B.4.4. Extrapolation of table entries}

As mentioned above, $\Delta t$ is the value that is stored in the tables. Unfortunately it does not depend continuously on the parameters $t_{E}, h_{E}$ or $v_{E}$ (cf. Fig. B.6), where a small change (e.g. from $t_{E}$ to $t_{E}^{\prime}$ of edge $I$ ) results in an unsteady change of $\Delta t$. The usual technique of interpolating between neighboring table entries can therefore not be applied here.

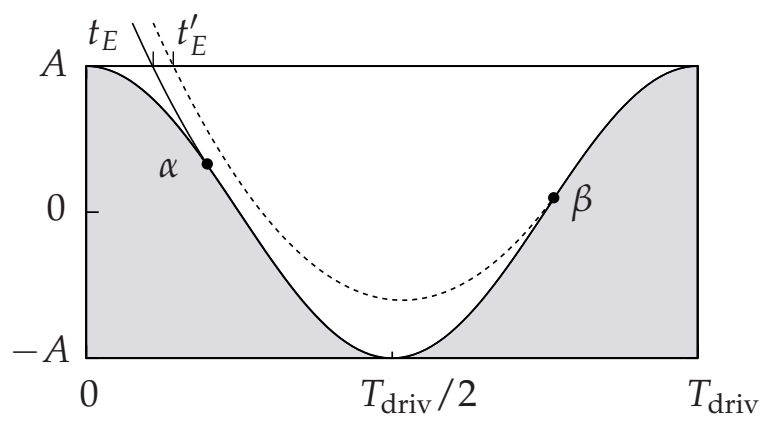

Fig. B.6 The time $\Delta t$ does not depend continuously on $t_{E}$ or $v_{E}$. A small increase from $t_{E}$ to $t_{E}^{\prime}$ results in a discontinuous jump in $\Delta t$. An interpolation between neighboring table entries is therefore not possible. 
The technique of choice is to store not only the next collision time, but also the partial derivatives with respect to the two parameters at the base point. Then we can perform a linear extrapolation around the base point, which is, in contrast to the interpolation, at least of reasonable quality as long as we are not extrapolating across the discontinuity. For edge $I$ and $I I$ the partial derivatives $\frac{\partial \Delta t}{\partial t_{E}}$ and $\frac{\partial \Delta t}{\partial v_{E}}$ at the respective base points are stored, while for edge III $\frac{\partial \Delta t}{\partial h_{E}}$ and $\frac{\partial \Delta t}{\partial v_{E}}$ are stored. To calculate the partial derivative of $\Delta t(P)$, where $P$ is either $t_{E}, h_{E}$ or $v_{E}$, the implicit derivative is used. Differentiation of

$$
\tilde{s}_{\text {rel }}(P):=s_{\text {rel }}(\Delta t(P), P) \equiv 0
$$

with respect to $P$ yields

$$
0=\frac{\partial \tilde{s}_{\text {rel }}(P)}{\partial P}=\frac{\partial s_{\text {rel }}(\Delta t(P), P)}{\partial \Delta t} \frac{\partial \Delta t(P)}{\partial P}+\frac{\partial s_{\text {rel }}(\Delta t(P), P)}{\partial P} .
$$

Hence, we have the familiar expression,

$$
\frac{\partial \Delta t(P)}{\partial P}=-\frac{\frac{\partial s_{\mathrm{rel}}}{\partial P}}{\frac{\partial s_{\mathrm{rel}}}{\partial \Delta t}}
$$

and we can calculate and store all of the required derivatives for each parameter and each edge.

\section{B.4.5. Selection of the appropriate root}

Theoretically, there is no limit for the number of roots of Eq. (B.7). However, due to the limitation of the predicted time to $0 \leqslant \Delta t \leqslant T_{\text {driv }}$ we can find, at most, four roots within that interval. This can easily be verified by differentiating the respective expression. Only two of them are physically relevant, because the others would correspond to a collision of the inner side of the particle with the backside of the wall. This can be see in Fig.B.7, where a trajectory with three roots is shown and the intersection at point 2 would be unphysical. It is also visible in this sketch that one single particle can never collide at point 3 with the wall, because it would have already collided at point 1 . However, if there is more than one particle it is possible that a particle-particle collision, which takes place in the time between intersection 2 and 3 , drives the particle to that trajectory with the collision at point 3 . As stated earlier, the wanted solution is still the one which is closest in time after the particles' present time. Because we are using the edges of the frame around one period of the wall motion as the origin, we have to store for each trajectory, if applicable, not only one but both physical roots 1 and 3 .

The time $t_{\mathrm{min}}$, which is the interval length during the search of the bracketing bounds, denotes the resolution limit of two different roots. This means that it is possible for a length of time $t_{\min }$ that the particle is located behind the wall. If during this situation another particle collides with it, we are able to safely detect 


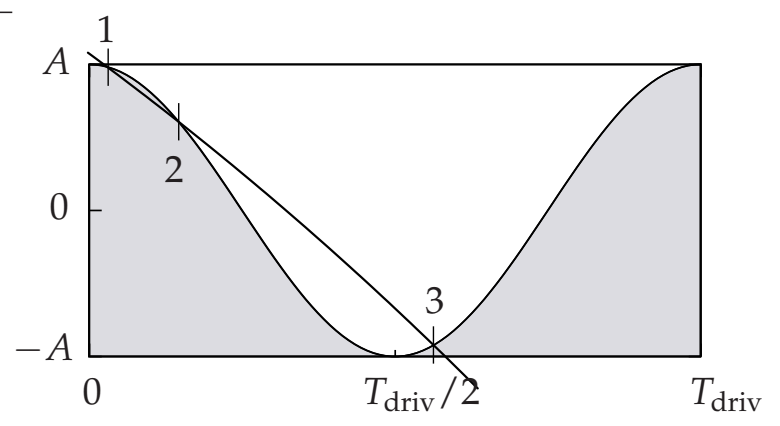

Fig. B.7 Typical trajectory with three points of intercept with the sinusoidal wall motion. Root 1 and 3 correspond to physically valid collisions with the wall, but the intersection at 2 is unphysical since this would correspond to a collision of the interior of the particle with the back side of the wall. In order for a particle to collide with the wall at point 3 a particle-particle collision between the time of points 2 and 3 would be necessary to drive the particle back into this trajectory.

this situation. This is important, because after the collision the root which is closest in time would correspond to an unphysical collision. To solve this problem we perform an instantaneous wall collision as soon as we detect this situation, in order to regain the missed wall collision. Therefore, we also have to store both unphysical roots in the table to be able to detect this situation. Through the tabulation of the trajectories we project each particle's motion to a discrete set of trajectories and due to the finite resolution it is even more likely that this situation can occur.

As the times $\Delta t$ of all four roots are stored in the table, we show a simple scheme which is used to decide during runtime, on the basis of the particles' present time, which root has to be used or if an instantaneous collision is inevitable. This can be compressed into a single if-instruction:

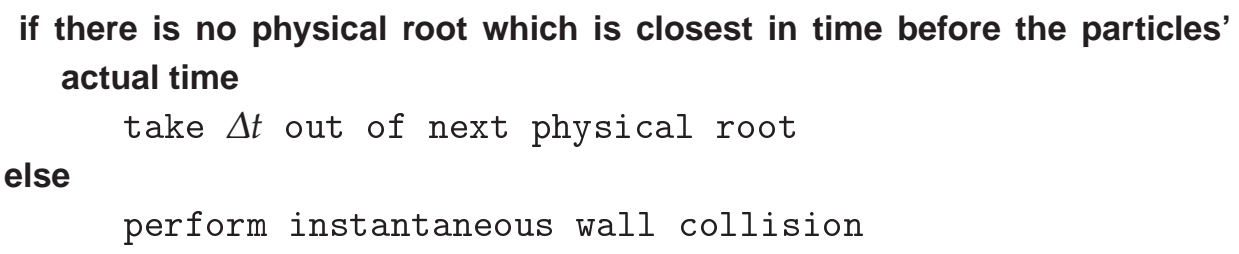

\section{B.4.6. Precision and applicability}

The method presented in this work to simulate components with trajectories of arbitrary functional form is mathematically not exact as EDMD simulations usually are, but it is a numerical approximation with an additional tabulation of the values.

The precision of our method therefore increases with a decreasing periodic time. Furthermore, the precision increases with a decreasing distance between the maximum and minimum value of the trajectory if the amount of storage space for the tables is kept constant.

It is thus appropriate to choose a coordinate system which minimizes this distance as shown. Assuming that a trajectory is always a steady function (which is quite 
reasonable for macroscopic systems), this equivalently minimizes the range of the function. This method therefore is suitable to simulate components of the system that have a periodic time much smaller than the total simulation time. Additionally, the range of motion of the component has to be small compared to the overall system size.

These restrictions are well fulfilled for an oscillating wall that is moving sinusoidally, in the parameter space that is also accessible by experiments. The precision increases in this case with a smaller oscillation period and amplitude. The coordinate system is chosen such that one axis points in the same direction as the amplitude vector of the oscillating wall.

\section{B.4.7. Initialization of the look up table}

The initialization of the look up table can be done either successively, during the run of the simulation, or an initialization process can prepend the simulation, and initialize the whole table at once. The first procedure is preferred if every single calculation is very time consuming and only few table entries will be used during the simulation. The disadvantage is that every time the table is used it must be checked if the entry is already calculated. The second method is better if the use of the table entries is distributed as widely as possible. For our purpose it is preferable to initialize the table at the beginning because the system is chaotic and therefore all possible movements will occur during the simulation. During runtime we have only to decide which table entry is the appropriate one.

\section{B.5. Essential numerical improvements}

In the previous section we have seen the essential concepts and methods that are necessary for simulating components with trajectories of arbitrary functional form, shown for a sinusoidally moving wall, with the help of look-up tables. In this section we will additionally show some essential numerical enhancements in order to couple this approximated dynamic of the wall collisions to the mathematically exact calculation of particle-particle interactions in the bulk.

\section{B.5.1. Particle positions}

To calculate the next particle position in EDMD simulations the particle is evolved along its trajectory for the time $\Delta t$. In the case of a collision with the wall, $\Delta t$ is determined with the help of the look up tables as described before and thus is an approximated value. Therefore, it is probable that the particle will be situated slightly, but more so than is caused by machine imprecision, off the wall. With a higher table resolution we can improve the precision of $\Delta t$ and thereby the accuracy of positioning the particle, but we never rid ourselves of this inaccuracy. Instead, we have to improve the agreement in the particle position between the propagation 
along the particle trajectory and along the trajectory out of the table for which the collision time was calculated. We solve this discrepancy by considering the time $\Delta t$ in this case to be exact, but the position out of the propagation along the trajectory as faulty. We know exactly the position of the wall at time $\Delta t$, thus, we can directly set the particle position to this value.

\section{B.5.2. Extra table after wall collisions}

The possible positions where a particle can be situated inside the range of action of the wall is infinite. This directly led to the main idea of tabulating trajectories and using the edges of a frame around one period as the origin. However, at the moment of the wall collision we know exactly the position of the respective particle and with the previous subsection B.5.1 this is even ensured within machine precision.

We introduce an extra table where the origin of $\Delta t$ is located directly on the wall. Thereby, the precision of $\Delta t$ is higher in these cases because we can avoid the back and forth propagation of the particle, which is favorable because the forth propagation is on one of the discrete trajectories of the table. We remark that, in analogy to the edges I, II and III introduced in Fig. B.4, the edge of this specialized table looks like the cosine-shaped driving function. The origin thus is the intersection of a particle trajectory with the wall trajectory. This table is always used for predictions after wall collisions and its two parameters are $t_{E_{i}}$ and $v_{E_{i}}$ similar to edge $I$ and $I I$, respectively, as introduced in section B.4.2.

\section{B.5.3. Tabulation of the relative velocity}

Not only the position but also the velocity $v_{P}^{\prime}$ of the particle after the wall collision has to be calculated. It is given by

$$
v_{P}^{\prime}=v_{w}+v_{\text {rel }}
$$

with the velocity of the wall $v_{w}$ and the relative velocity of the particle with respect to the wall $v_{\text {rel }}$ where

$$
v_{\text {rel }}=v_{w}-v_{P}
$$

with the velocity $v_{P}$ of the particle before the collision.

To perform a physically sensible wall collision, the relative velocity $v_{\text {rel }}$ has to be strictly larger than zero. Especially when the values of the wall velocity $v_{w}$ and the particle velocity $v_{P}$ are very close to each other, the sign of Eq. (B.19) can be negative when evaluated at the approximated time $\Delta t$. This is caused by the error which results from the linear extrapolation of $\Delta t$. From the physical point of view these are trajectories that run nearly parallel to the wall and gently touch it.

To prevent $v_{\text {rel }}$ being smaller than zero we do not use Eq. $(\overline{B .19)}$ to calculate it. Instead, we calculate $v_{\text {rel }}$ as

$$
v_{\text {rel }}(P):=\frac{\partial s_{\text {rel }}(\Delta t(P), P)}{\partial \Delta t(P)}
$$


and the partial derivatives of $v_{\text {rel }}$ with respect to the parameter $P$ by explicit differentiation of Eq. (B.20), which leads to

$$
\frac{\partial v_{\text {rel }}(P)}{\partial P}=\frac{\partial^{2} s_{\text {rel }}(\Delta t(P), P)}{\partial \Delta t(P)^{2}} \frac{\partial \Delta t(P)}{\partial P}+\frac{\partial^{2} s_{\text {rel }}(\Delta t(P), P)}{\partial P \partial \Delta t(P)} .
$$

With the knowledge of $v_{\text {rel }}$ and its partial derivatives, we are able to perform a linear extrapolation in the same way as was done for the calculation of $\Delta t$. For convenience we can store these values additionally in the look up table, because we are applying the discretization scheme to calculate $\Delta t$ also for $v_{\text {rel }}$ now.

With this method it is possible to ensure that $v_{\text {rel }}$ is larger than zero, because this can be validated for any possible value out of the look up table, already at the initialization process, as we will see in the next subsection.

\section{B.5.4. Extrapolation across discontinuities}

The discontinuous dependence of $\Delta t$ upon the parameters $t_{E}, v_{E}$ or $t_{E}, h_{E}$ was the reason why we introduced the partial derivative in section B.4.4 to extrapolate table entries. A zoom into our look-up table of, e.g., edge I at such a discontinuity is shown in Fig. B.8, where the solid black line is the physical discontinuity, a square represents one cell of the table, and the cross marks its midpoint. This is the point whose physical properties are used for calculating the values $\Delta t, v_{\text {rel }}$ and the respective partial derivates of this cell. Within such a single cell the values $\Delta t$ and $v_{\text {rel }}$ are extrapolated with the respective partial derivatives. As is clearly seen for the hatched cells of Fig. B.8, an extrapolation across the discontinuity can lead to unphysical results.

In order to avoid these unphysical results at the initialization process of the hatched cells we verify that the value out of this table entry fulfills - at any point within the cell - the following physical conditions:

$$
\begin{gathered}
\Delta t>0 \\
v_{\text {rel }}>0 .
\end{gathered}
$$

It is sufficient to verify these two requirements are met at the four corners of each cell. This results from the linear extrapolation, which makes both functions (B.22) and (B.23) monotonic within one cell. Therefore, they take the maximum value at the border of the respective cell. Taking both dimensions into account, the overall maximum has to be located on one of the four corners. If one of these two conditions is violated, we artificially change the derivatives slightly to sufficiently small values in order to fulfill them again. In the simplest manner of initialization, the derivatives are set to zero. 


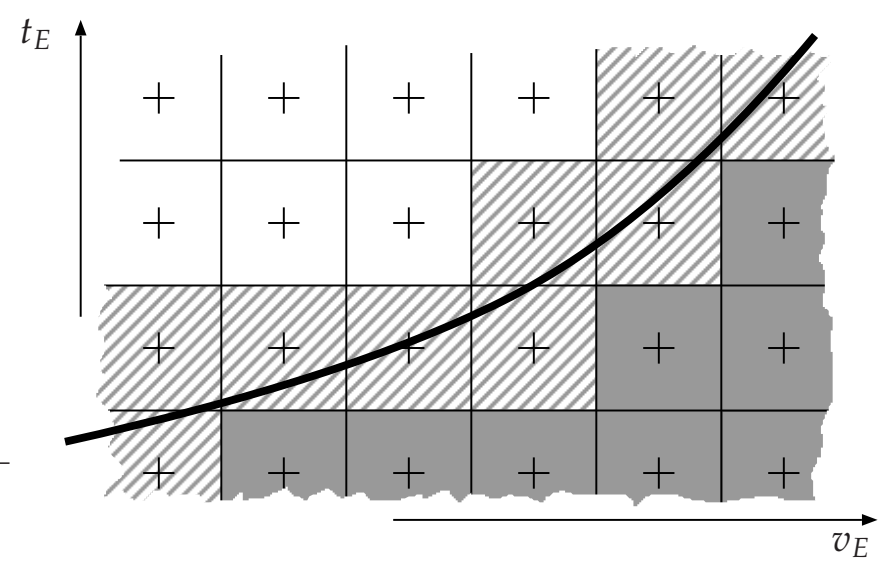

Fig. B.8 Zoom into a look-up table at a discontinuity, where the solid black line is the physical discontinuity, a square represents one entry of the table and the cross marks its midpoint used for calculation. Within a cell, $\Delta t$ and $v_{\text {rel }}$ are extrapolated with the respective partial derivatives. This can lead to unphysical results when extrapolating across the discontinuity. By slightly changing the partial derivatives, the physical correctness of the extrapolated values is assured.

\section{B.5.5. Assuring a consistent description between collision and escape}

As discussed before, it is necessary but also favorable to limit the maximum predictable time $\Delta t$ out of the table to values within one period only. In principle, it would be possible to store the exceptions out of Sec. B.3.3.2 directly in the table, but due to a kind of leverage effect, the error of $\Delta t$ would increase with larger $\Delta t$ up to a uselessness of the predicted time. Therefore, a second query with different initial conditions ensures the accuracy of the result as seen before.

Furthermore, this method gives rise to another discontinuity where a particle, initially located inside the range of action of the wall, either has a collision within the respective period or it escapes by crossing the edge $I$ from below. A trajectory not leading to a collision within the respective period is represented in the table with the value $\Delta t=\infty$ (cf. Fig. B.9a). We therefore have to ensure, for any cell where the value $\Delta t$ of its midpoint is $\infty$ (shaded cells in Fig. B.9a) that also at the corners of that cell the escape condition of crossing edge $I$ is fulfilled. This would otherwise lead to an inconsistency where the table claims that the particle does not collide within the respective period, but it would not be possible to calculate the time when it reenters the range of action of the wall because physically it never left it. In these cases we have to ensure

$$
\Delta t<\infty
$$

For these cells therefore it is not the midpoint that defines the physical properties, but rather one of the corners (circles in Fig. B.9b), for which condition (B.24) is fulfilled. The extrapolation scheme for these cells (hatched cells in Fig. B.gb) would differ during runtime because the origin for the extrapolation is different. We therefore extrapolate the value $\Delta t$ from this shifted position, already during the initialization 
process, back to the usual midpoint which is then stored as usual.

a

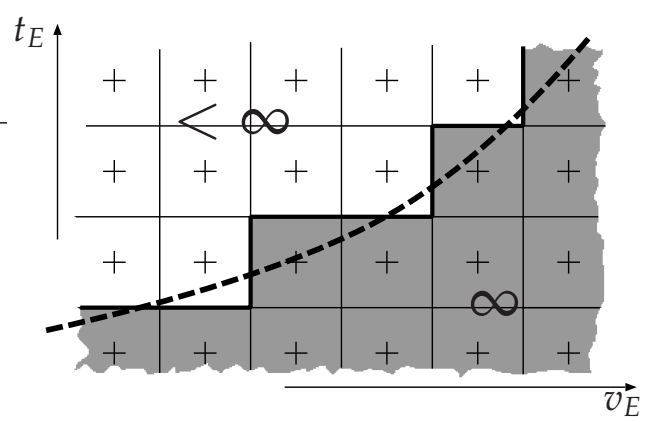

b

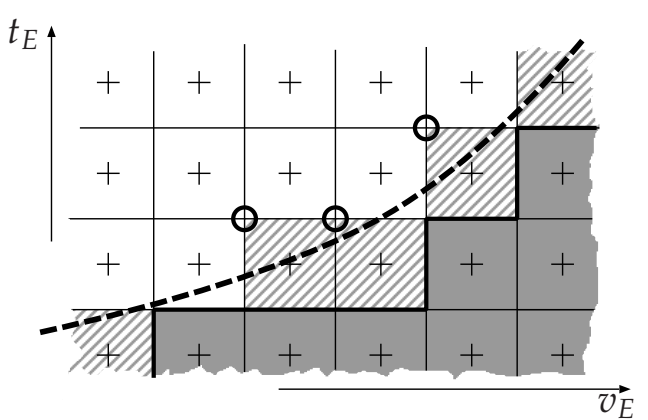

Fig. B.9 Discretization (solid black line) of the physical discontinuity (dashed line) $\mathbf{a}$ before and $\mathbf{b}$ after changing the reference point (circles in b) for the hatched cells.

\section{B.5.6. Instantaneous events as protection against unphysical behavior}

The most problematic case in any event-driven simulation is to avoid overlaps between two particles or between a particle and a wall. As soon as such an overlap occurs, the solution of Eq. (B.I) or (B.4) yields unphysical behavior. For two overlapping particles this would result in a collision on the inner side of the particles. Once overlapping, the overlap would, formally, be persistent so that they are entangled forever and then could pass through each other. Overlaps, nevertheless, are hardly avoidable due to roundoff errors. Precisely half of the results of Eq. (B.3) yield overlapping particles due to the finite number representation. The task therefore is not to prevent overlaps but to handle them.

Unphysical collisions with the resulting entanglement can easily be prevented by only accepting physical solutions which can be identified by the appropriate change of sign in the respective equations. For particles in a homogeneous gravity field e. g., it is even analytically possible to pick the right solution without any additional calculation of the wrong solution or the derivative.

To prevent particles passing through each other requires, however, more effort. The approach is to perform minimally invasive changes in the dynamics which are sufficient to avoid unphysical behavior. Depending on the relative velocity of the overlapping particles we act differently. If the particles become separated in the future we do literally nothing, because the overlap will decrease and finally disappear. If the particles are overlapping and approaching each other, then this situation leads to an increase of the overlap. To prevent this, we perform an instantaneous elastic collision. This means that we perform a collision at the current time for the two respective particles with their present relative velocity (which is like pressing the pause button for all other particles). Since the relative velocity is negative, the particles approach each other and there is a collision at their outer rims, but due to the 
overlap their radii are slightly decreased. Also a chain of overlapping particles can be unleashed with this method because they perform consecutive instantaneous collisions until the outmost particles are uncaged. Similarly, we can successfully handle overlaps of particles with the wall where the overlap can be even larger because the solution of Eq. (B.7) is only approximated. Therefore, grazing shots of particles with the wall do not cause problems in the simulation.

\section{B.6. Comparison of performance}

\section{B.6.1. Comparison of the simple algorithm with tabulation}

To demonstrate the benefit of the tabulation method we compare it with the simple algorithm described in Sec. B.3, which finds the root with a brute force method for one calculation of the next collision time $\Delta t$. As mentioned before, the complexity of the simple algorithm is linearly dependent on the number of intervals which resolve one period of the driving function. This is confirmed directly by simulations (circles in Fig.(B.10).

The average runtime for one calculation of the next wall collision event is plotted in arbitrary units as a function of the number of intervals. The circles denote the simulation, and the solid line is a linear fit through all simulation points. In contrast, the runtime complexity of the tabulation method is independent of the number of intervals and is shown as dashed line in the figure, with a constant mean value of only 0.29 in these units. We clearly see that with the tabulation method the runtime performance is superior by more than two orders of magnitude in comparison with a brute force root-finding method with a reasonable resolution of the driving function.

\section{B.6.2. Comparison of overall performance}

In the previous subsection we saw an increase of the runtime performance by two orders of magnitude for the calculation of a single wall collision event gained by the use of the tabulation method. However, in a real simulation not all computational effort is caused by collisions with a wall. Therefore, we now compare in a real simulation the runtime performance of the tabulation method with a brute force root-finding method. The system which is simulated here is the same as that was used in (Fingerle et al., 2008).

In Fig. B.11, we show the required computation time which is necessary for simulating a certain physical time for the brute force method (circles) and the efficient tabulation method (squares) for different numbers of particles. We clearly see that the runtime of the latter is decreased by approximately one order of magnitude. This is quite striking and verifies that implementation of the tabulation method is worth the effort. This increase in overall performance naturally depends on the geometry and other details of the simulation. For better comparison also the calculation 


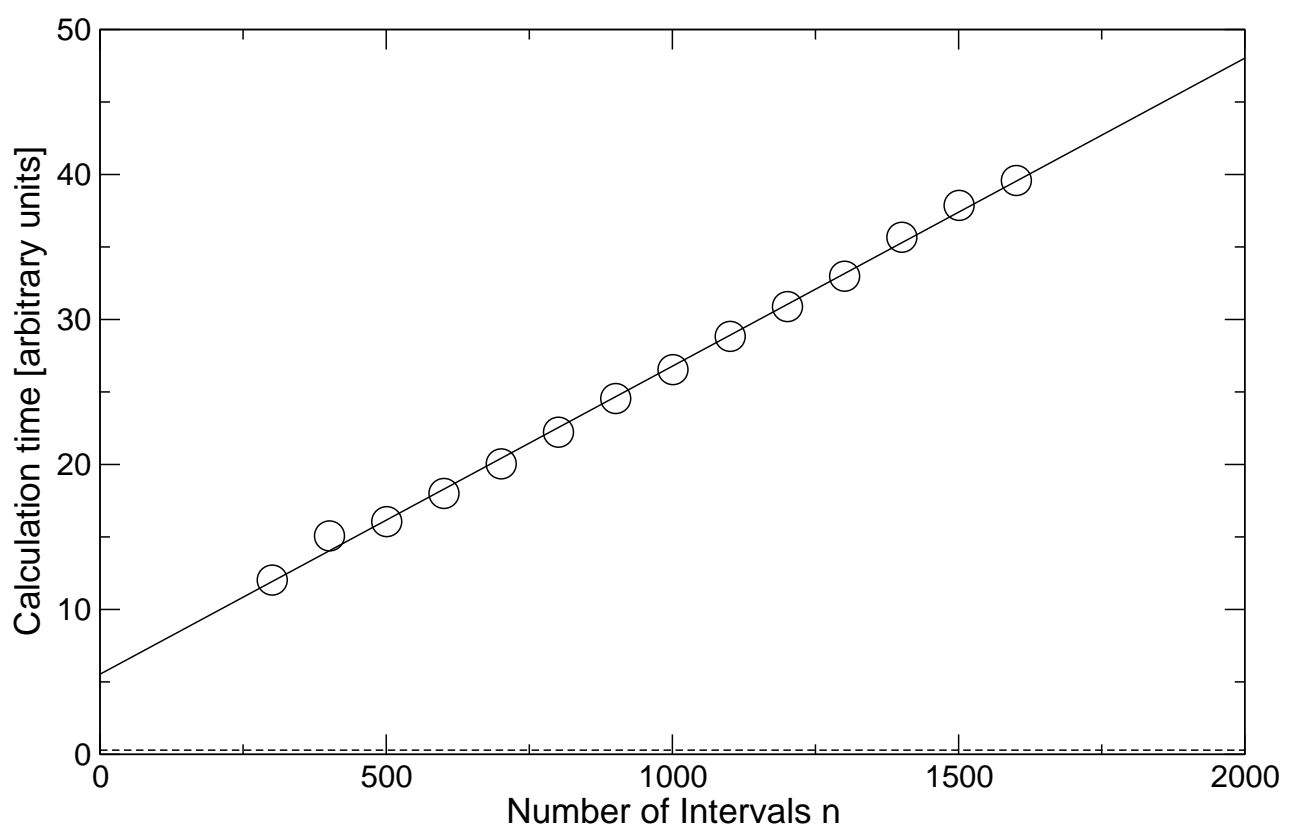

Fig. B.10 The average calculation time with the brute force algorithm (circles) for one calculation of the next wall collision event is measured in simulations. The solid line denotes a linear fit through these values, which clearly shows the linear dependence on the number of intervals. The dashed line with a constant value of 0.29 in the respective units shows the calculation time for the tabulation method which is during runtime independent of the time resolution. A performance boost of more than two orders of magnitude can be obtained for a reasonable number of intervals $n$.

time of a TDMD simulation method is shown (diamonds), which of course is always large when compared with the EDMD method independent of the choice of the root-finding method.

\section{B.7. Summary}

A simple algorithm is presented to simulate flat walls in a system with periodic trajectories in event-driven molecular dynamics simulations and the details are shown for the example of a sinusoidally moving wall. It is based on a root finding method, which is briefly reviewed in Sec.B.3.1. It is a combination of the Newton-Raphson method and Bisection method as described in Press et al. (1992). The most expensive task in this algorithm is to find a unique bracketing bound around the root, which leads to a search algorithm with linear complexity in the inverse time resolution. Consequently, we introduced a tabulation scheme to store the solutions, which are the times for the next collisions with the wall in order to improve the efficiency. While the tabulation of functions is a standard method in computer science, we implemented it in a nontrivial manner where every table entry represents a trajectory. Each trajectory is uniquely characterized by a point in space-time on the trajectory 


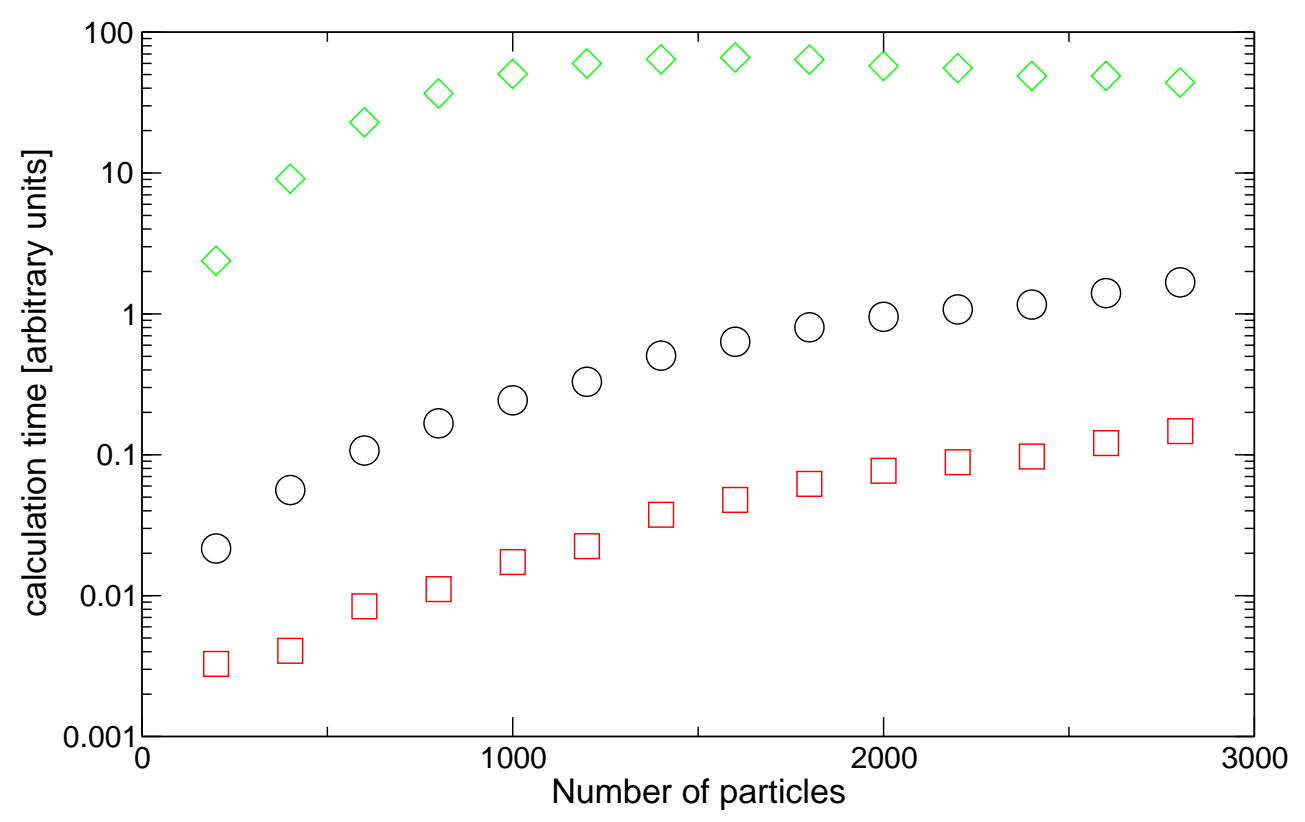

Fig. B.11 The decrease in the calculation time from the brute force method (circles) to the tabulation method (squares), which is shown for different numbers of particles, of about one order of magnitude clearly demonstrates the high efficiency of the tabulation method. For comparison also the TDMD simulation method is shown (diamonds). The simulated system is an isochore system of wet granular matter (Fingerle et al., 2008), in which for larger numbers of particles the system temperature is decreasing due to increased dissipation. The integration steps in TDMD then can be larger and the calculation time even decreases with increasing number of particles, whereas the calculation time in EDMD still increases due to the higher collision frequency.

and the velocity at that point. Therefore, we introduced a frame around one period of the sinusoidally shaped trajectory in the space-time plot. An intersection of the trajectory with an edge of the frame defines the origin for which the solution is stored in the look-up table. A separate two-dimensional table for each edge is set up with the first parameter completing the definition of the space-time point together with the condition of the edge and the velocity at that point as second parameter.

We additionally show all of necessary methods needed to ensure the logical consistency of the numerical computation in view of the inevitable finite table resolution. Because the time for the next collision does not depend continuously on the two parameters of the table, we also store the partial derivatives to perform a linear extrapolation between different table entries. This can, however, still lead to unphysical results when extrapolating across a discontinuity and therefore we adjust the derivatives to fulfill Eq. (B.22) and (B.23) in order to avoid unphysical predictions. To ensure the consistency of the predicted wall collision time and instantaneous wall velocity, the relative velocity after the wall collision is tabulated in the same way.

The described tabulation method is a powerful instrument with very good performance. The sinusoidal driving function becomes particularly important when com- 
paring simulations with experiments, because, due to mechanical limitations, most of the experiments are performed with sinusoidal driving. This method has significant potential to numerically study the behavior of particulate systems like granular materials or powders, and thus advance the growing field of statistical physics far from equilibrium. Interesting topics to study include fluidization transitions (Scheel et al., 2004) or granular ratchets, where high performance of the simulation is required and sinusoidal shaking is desirable. 



\section{Bibliography}

B. Alder and T. Wainwright. Phase Transition for a Hard Sphere System. The Journal of Chemical Physics, 27(5):1208-1209, 1957.

B. Alder and T. Wainwright. Studies in Molecular Dynamics. I. General Method. The Journal of Chemical Physics, 31(2):459-466, 1959.

M. Allen and D. Tildesley. Computer Simulation of Liquids. Oxford University Press, 1987.

J. Andrews, S. Shakkottai, R. Heath, N. Jindal, M. Haenggi, R. Berry, D. Guo, M. Neely, S. Weber, S. Jafar, and A. Yener. Rethinking Information Theory for Mobile Ad Hoc Networks. IEEE Commun. Magazine, 46(12):94-101, 2008.

I. S. Aranson and L. S. Tsimring. Patterns and collective behavior in granular media: Theoretical concepts. Reviews of Modern Physics, 78(2):641, 2006.

A. Ardekani and R. Rangel. Collision of a sphere onto a wall coated with a liquid film. arXiv:0809.3770, 2008. video: http://hdl.handle.net/1813/11394.

D. Armbruster, B. Nicolaenko, N. Smaoui, and P. Chossat. Symmetries and dynamics for 2-D Navier-Stokes flow. Physica D: Nonlinear Phenomena, 95(1):81 - 93, 1996.

V. I. Arnold and B. A. Khesin. Topological Methods in Hydrodynamics. Annual Review of Fluid Mechanics, 24(1):145-166, 1992.

C. Bain. Nanostructures: Drip painting on a hot canvas. Nature Nanotechnology, 2(6): 344-345, 062007.

M. Balazs, J. Quastel, and T. Seppalainen. Scaling exponent for the Hopf-Cole solution of KPZ/Stochastic Burgers. Arxiv preprint arXiv:0909.4816, 2009.

A.-L. Barabási and H. E. Stanley. Fractal concepts in surface growth. Cambridge University Press, 1995.

A. Baskaran, J. W. Dufty, and J. Brey. Transport coefficients for the hard-sphere granular fluid. Physical Review E (Statistical, Nonlinear, and Soft Matter Physics), 77(3):031311, 2008. 
J. Becker, G. Gr

"un, R. Seemann, H. Mantz, K. Jacobs, K. Mecke, and R. Blossey. Complex dewetting scenarios captured by thin-film models. Nature Materials, 2(1):59-63, 2003.

B. Belousov. A periodic reaction and its mechanism. In R. Field and M. Burger, editors, Oscillations and Travelling Waves in Chemical Systems, New York, 1985, page 605-613, 1951.

B. Belousov. An oscillating reaction and its mechanism. Sborn. referat. radiat. med., page 145, 1959.

L. Bergmann and C. Schaefer. Lehrbuch der Experimentalphysik, Bd. 1. Mechanik, Relativität, Wärme. W de Gruyter, 1998.

F. Bertrand, L.-A. Leclaire, and G. Levecque. DEM-based models for the mixing of granular materials. Chemical Engineering Science, 60(8-9):2517-2531, 2005. 5th International Symposium on Mixing in Industrial Processes (ISMIP5).

A.-L. Biance, C. Clanet, and D. Quéré. Leidenfrost drops. Physics of Fluids, 15(6): $1632-1637,2003$.

F. Black and M. Scholes. The pricing of options and corporate liabilities. Journal of political economy, 81(3):637, 1973 .

D. L. Blair and A. Kudrolli. Clustering transitions in vibrofluidized magnetized granular materials. Phys. Rev. E, 67(2):021302, Feb 2003.

L. Bocquet, Élisabeth Charlaix, and F. Restagno. Physics of humid granular media. Comptes Rendus Physique, 3(2):207 - 215, 2002.

N. N. Bogoliubov. Kinetic Equations. Journal of Physics USSR, 10(3):265-274, 1946.

N. N. Bogoliubov and K. P. Gurov. Kinetic Equations in Quantum Mechanics. Journal of Experimental and Theoretical Physics, 17(7):614-628, 1947.

L. Boltzmann. Vorlesungen über Gastheorie. Leipzig, 1896.

M. Born and H. S. Green. A General Kinetic Theory of Liquids I. The Molecular Distribution Functions. Proc. Roy. Soc. A, 188:10-18, 1946.

J.-P. Bouchaud and A. Georges. Anomalous diffusion in disordered media: Statistical mechanisms, models and physical applications. Physics Reports, 195(4-5):127-293, 1990.

M. Bowker. Surface science: The going rate for catalysts. Nature Materials, 1(4):205-206, 2002.

J. Brey, J. W. Dufty, C. S. Kim, and A. Santos. Hydrodynamics for granular flow at low density. Phys. Rev. E, 58(4):4638-4653, Oct 1998. 
J. Brey, M. Ruiz-Montero, and F. Moreno. Hydrodynamics of an open vibrated granular system. Phys. Rev. E, 63(6):061305, May 2001.

J. J. Brey and M. J. Ruiz-Montero. Hydrodynamic character of the nonequipartition of kinetic energy in binary granular gases. Physical Review E (Statistical, Nonlinear, and Soft Matter Physics), 8o(4):041306, 2009.

N. Brilliantov and T. Pöschel. Kinetic Theory of Granular Gases. Oxford Univ. Press, Oxford, UK, 2004.

N. Brilliantov and T. Pöschel. Granular Gases with Impact-Velocity-Dependent Restitution Coefficient, pages 100-124. Springer Berlin / Heidelberg, 2001.

N. Brilliantov, T. Pöschel, W. Kranz, and A. Zippelius. Translations and Rotations Are Correlated in Granular Gases. Physical Review Letters, 98(12):128001, 2007.

D. Brockmann. Superdiffusion in Scale-Free Inhomogeneous Environments. PhD thesis, University Goettingen, 2003.

R. Brown. A brief Account of Microscopical Observations made in the Months of June, July, and August, 1827, on the Particles contained in the Pollen of Plants; and on the general Existence of active Molecules in Organic and Inorganic Bodies. Philosophical Magazine, 4:161-173, 1828 .

R. Brown. Additional Remarks on Active Molecules. Philosophical Magazine, 6:161-166, 1829.

J. M. Burgers. The Nonlinear Diffusion Equation. D. Reidel Publishing Company, Boston, 1974 .

J. M. Burgess, C. Bizon, W. D. McCormick, J. B. Swift, and H. L. Swinney. Instability of the Kolmogorov flow in a soap film. Phys. Rev. E, 60(1):715-721, Jul 1999.

H.-J. Butt and M. Kappl. Normal capillary forces. Advances in Colloid and Interface Science, 146(1-2):48 - 60, 2009.

C. T. Campbell, S. C. Parker, and D. E. Starr. The Effect of Size-Dependent Nanoparticle Energetics on Catalyst Sintering. Science, 298(5594):811-814, 2002.

J. Carrillo, T. Poeschel, and C. Saluena. Granular hydrodynamics and pattern formation in vertically oscillated granular disk layers. Journal of Fluid Mechanics, 597:119-144, 2008.

A. Caspi, R. Granek, and M. Elbaum. Diffusion and directed motion in cellular transport. Phys. Rev. E, 66(1):011916, Jul 2002.

J. Chakrabarti, J. Dzubiella, and H. Löwen. Reentrance effect in the lane formation of driven colloids. Phys. Rev. E, 70(1):012401, Jul 2004. 
S. Chapman and T. Cowling, editors. The Mathematical Theory of Non-Uniform Gases. Cambridge University Press, 3rd edition, 1970.

Z.-M. Chen and W. G. Price. Time dependent periodic Navier-Stokes flows on a twodimensional torus. Communications in Mathematical Physics, 179(3):577-597, 1996.

Z.-M. Chen, M. Ghil, E. Simonnet, and S. Wang. Hopf Bifurcation in Quasi-Geostrophic Channel Flow. SIAM Journal on Applied Mathematics, 64(1):343-368, 2003.

X. Cheng, G. Varas, D. Citron, H. M. Jaeger, and S. R. Nagel. Collective Behavior in a Granular Jet: Emergence of a Liquid with Zero Surface Tension. Phys. Rev. Lett., 99(18): 188001, Nov 2007.

X. Cheng, L. Xu, A. Patterson, H. M. Jaeger, and S. R. Nagel. Towards the zero-surfacetension limit in granular fingering instability. Nature Physics, 4(3):234-237, Mar 2008.

M. Clerc, P. Cordero, J. Dunstan, K. Huff, N. Mujica, D. Risso, and G. Varas. Liquidsolid-like transition in quasi-one-dimensional driven granular media. nature physics, 4: 21, 2008.

P. Cundall and O. Strack. A discrete numerical model for granular assemblies. Geotechnique, 29:47-65, 1979 .

J. H. Cushman, M. Park, and D. O'Malley. Chaotic dynamics of super-diffusion revisited. Geophys. Res. Lett., 36, 042009.

G. D'Anna. Observing brownian motion in vibration-fluidized granular matter. Nature, 424(6951):909-912, 2003.

P. de Gennes, F. Brochard-Wyart, and D. Quere. Capillarity and Wetting Phenomena: Drops, Bubbles, Pearls, Waves. Springer, New York, 2003.

P. Deltour and J.-L. Barrat. Quantitative Study of a Freely Cooling Granular Medium. Journal de Physique I, 7(1):137-151, jan 1997.

A. Dhanarajan and R. Bandyopadhyay. An energy-based population-balance approach to model granule growth and breakage in high-shear wet granulation processes. AAPS PharmSciTech, 8(3), 2007.

A. Donev, S. Torquato, and F. H. Stillinger. Neighbor list collision-driven molecular dynamics simulation for nonspherical hard particles. I. Algorithmic details. J. Comp. Phys., 202(2):737-764, January 2005a.

A. Donev, S. Torquato, and F. H. Stillinger. Neighbor list collision-driven molecular dynamics simulation for nonspherical hard particles.: II. Applications to ellipses and ellipsoids. J. Comp. Phys., 202(2):765-793, January 2005b.

B. Dubrulle and U. Frisch. Eddy viscosity of parity-invariant flow. Phys. Rev. A, 43(10): 5355-5364, May 1991. 
J. W. Dufty and J. J. Brey. Brownian motion in a granular fluid. New Journal of Physics, 7:20, 2005 .

J. Duran. Free Cooling of the One-Dimensional Wet Granular Gas. Physical Review Letters, 97(1):018001, 2006.

J. Duran. Sands, Powders, and Grains. Springer, New York, 2000.

S. F. Edwards and D. R. Wilkinson. The Surface Statistics of a Granular Aggregate. Proceedings of the Royal Society of London. Series A, Mathematical and Physical Sciences, 381(1780):17-31, 1982.

J. Eggers. Sand as Maxwell's Demon. Phys. Rev. Lett., 83(25):5322-5325, Dec 1999.

A. Einstein. Über die von der molekularkinetischen Theorie der Wärme geforderte Bewegung von in ruhenden Flüssigkeiten suspendierten Teilchen. Annalen der Physik, 322(8):549560, 1905 .

M. Elbahri, D. Paretkar, K. Hirmas, S. Jebril, and R. Adelung. Anti-Lotus Effect for Nanostructuring at the Leidenfrost Temperature. Advanced Materials, 19(9):1262-1266, May 2007.

P. Eshuis, K. v. d. Weele, D. v. d. Meer, and D. Lohse. Granular Leidenfrost Effect: Experiment and Theory of Floating Particle Clusters. Physical Review Letters, 95(25): 258001, 2005 .

P. Eshuis, K. van der Weele, D. van der Meer, R. Bos, and D. Lohse. Phase diagram of vertically shaken granular matter. Physics of Fluids, 19(12):123301, 2007.

S. Esipov and T. Pöschel. The granular phase diagram. Journal of Statistical Physics, 86: $1385,1997$.

D. Evans, E. Cohen, and G. Morriss. Probability of second law violations in shearing steady states. Phys. Rev. Lett., 71(15):2401-2404, Oct 1993a.

D. Evans, E. Cohen, and G. Morriss. Probability of Second Law Violations in Shearing Steady States. Phys. Rev. Lett., 71(21):3616, Nov 1993b.

Farkas. Social behaviour: Mexican waves in an excitable medium. Nature, 419(6903):131, 2002.

A. Fingerle and S. Herminghaus. Unclustering Transition in Freely Cooling Wet Granular Matter. Physical Review Letters, 97(7):078001, 2006.

A. Fingerle and S. Herminghaus. Equation of state of wet granular matter. Physical Review E (Statistical, Nonlinear, and Soft Matter Physics), 77(1):011306, 2008.

A. Fingerle, K. Roeller, K. Huang, and S. Herminghaus. Phase transitions far from equilibrium in wet granular matter. New Journal of Physics, 10(5):053020 (10pp), 2008. 
R. Fisher. On the capillary forces in an ideal soil, correction of formulate given by W.B. Haines. Journal of Agricultural Science, 16:492-505, 1926.

J. J. Fox, R. F. Gilmour, and E. Bodenschatz. Conduction Block in One-Dimensional Heart Fibers. Phys. Rev. Lett., 89(19):198101, Oct 2002.

J. W. M. Frenken, P. M. J. Marée, and J. F. van der Veen. Observation of surface-initiated melting. Phys. Rev. B, 34(11):7506-7516, Dec 1986.

U. Frisch, B. Hasslacher, and Y. Pomeau. Lattice-Gas Automata for the Navier-Stokes Equation. Phys. Rev. Lett., 56(14):1505-1508, Apr 1986.

E. Fuchs and L. Wallenhorst. Projektpraktikum Physik: Feuchte granulare Materie. section 17.4, especially Fig. 44, 2008.

G. Gallavotti. Fluctuation theorem. Scholarpedia, 3(2):5904, 2008.

G. Gallavotti and E. Cohen. Dynamical Ensembles in Nonequilibrium Statistical Mechanics. Phys. Rev. Lett., 74(14):2694-2697, Apr 1995.

S. Gama, U. Frisch, and H. Scholl. The two-dimensional Navier-Stokes equations with a large-scale instability of the Kuramoto-Sivashinsky type: Numerical exploration on the Connection Machine. Journal of Scientific Computing, 6(4):425-452, 1991.

X. Gao and L. Jiang. Water-repellent legs of water striders. Nature, 432(7013):36, 2004.

R. Garcia-Rojo, H. Herrmann, and S. McNamara, editors. Powders and Grains, 2005. Balkema.

F. Gerl and A. Zippelius. Coefficient of restitution for elastic disks. Phys. Rev. E, 59(2): 2361-2372, Feb 1999.

D. Geromichalos, M. M. Kohonen, F. Mugele, and S. Herminghaus. Mixing and Condensation in a Wet Granular Medium. Phys. Rev. Lett., 90(16):168702, Apr 2003.

J. W. Gibbs. On the Equilibrium of Heterogeneous Substances, Collected works. Longmans, Green, New York, 2:1-4, 1928.

G. Giese and A. Zippelius. Collision properties of one-dimensional granular particles with internal degrees of freedom. Phys. Rev. E, 54(5):4828-4837, Nov 1996.

I. Goldhirsch and G. Zanetti. Clustering instability in dissipative gases. Phys. Rev. Lett., 70(11):1619-1622, Mar 1993.

A. Goldshtein and M. Shapiro. Mechanics of collisional motion of granular materials. Part 1. General hydrodynamic equations. J. Fluid Mech., 282:75, 1995.

A. Götzendorfer, J. Kreft, C. A. Kruelle, and I. Rehberg. Sublimation of a Vibrated Granular Monolayer: Coexistence of Gas and Solid. Phys. Rev. Lett., 95(13):135704, Sep 2005 . 
A. Götzendorfer, C. A. Kruelle, I. Rehberg, and D. Svenšek. Localized Subharmonic Waves in a Circularly Vibrated Granular Bed. Physical Review Letters, 97(19):198001, 2006a.

A. Götzendorfer, C.-H. Tai, C. A. Kruelle, I. Rehberg, and S.-S. Hsiau. Fluidization of a vertically vibrated two-dimensional hard sphere packing: A granular meltdown. Physical Review E (Statistical, Nonlinear, and Soft Matter Physics), 74(1):011304, $2006 \mathrm{~b}$.

J. S. A. Green. Two-dimensional turbulence near the viscous limit. Journal of Fluid Mechanics Digital Archive, 62(02):273-287, 1974.

M. Green. Markoff Random Processes and the Statistical Mechanics of Time-Dependent Phenomena. II. Irreversible Processes in Fluids. The Journal of Chemical Physics, 22(3): 398-413, 1954.

E. L. Grossman, T. Zhou, and E. Ben-Naim. Towards granular hydrodynamics in two dimensions. Phys. Rev. E, 55(4):4200-4206, Apr 1997.

A. Hager-Fingerle. Entropy Production and Phase Transitions far from Equilibrium with Emphasis on Wet Granular Matter. PhD thesis, University Goettingen, 2007.

H. Haken. Self-organization. Scholarpedia, 3(8):1401, 2008.

J. Hardy, Y. Pomeau, and O. de Pazzis. Time Evolution of a Two-Dimensional Classical Lattice System. Phys. Rev. Lett., 31(5):276-279, Jul 1973.

D. Herbison-Evans. Solving Quartics and Cubics for Graphics. In A. Paeth, editor, Graphics Gems V, pages 3-15, Chesnut Hill, 1995. Academic Press.

O. Herbst, P. Müller, M. Otto, and A. Zippelius. Local equation of state and velocity distributions of a driven granular gas. Phys. Rev. E, 70(5):051313, Nov 2004.

S. Herminghaus. Dynamics of wet granular matter. Adv. in Phys., 54(3):221-261, May 2005.

J. Hernández-Guzmán and E. R. Weeks. The equilibrium intrinsic crystal-liquid interface of colloids. Proceedings of the National Academy of Sciences, 106(36):15198-15202, 2009.

H. Hinrichsen. Nonequilibrium Critical Phenomena and Phase Transitions into Absorbing States. Advances in Physics, 49:815, 2000.

W. G. Hoover. Nonequilibrium Molecular Dynamics. Annual Review of Physical Chemistry, 34(1):103-127, 1983.

D. J. Hornbaker, R. Albert, I. Albert, A.-L. Barabasi, and P. Schiffer. What keeps sandcastles standing? Nature, 387:765, 1997.

J. J. Hoyt, M. Asta, and A. Karma. Method for Computing the Anisotropy of the SolidLiquid Interfacial Free Energy. Phys. Rev. Lett., 86(24):5530-5533, Jun 2001. 
C. Huan, X. Yang, D. Candela, R. Mair, and R. Walsworth. NMR experiments on a three-dimensional vibrofluidized granular medium. Phys. Rev. E, 69(4):041302, Apr 2004 .

K. Huang, G. Miao, P. Zhang, Y. Yun, and R. Wei. Shock wave propagation in vibrofluidized granular materials. Physical Review E (Statistical, Nonlinear, and Soft Matter Physics), 73(4):041302, 2006.

K. Huang, K. Roeller, and S. Herminghaus. Universal and non-universal aspects of wet granular matter under vertical vibration. submitted to EJP, 2009.

R. Imbihl and G. Ertl. Oscillatory Kinetics in Heterogeneous Catalysis. Chemical Reviews, 95(3):697-733, 1995.

C. Isenberg. The science of soap films and soap bubbles. Dover Publications, 1992.

E. Ising. Beitrag zur Theorie des Ferro- und Paramagnetismus. PhD thesis, Universität Hamburg, 1924.

S. M. Iveson, J. D. Litster, K. Hapgood, and B. J. Ennis. Nucleation, growth and breakage phenomena in agitated wet granulation processes: a review. Powder Technology, 117(1-2): 3-39, 2001.

H. M. Jaeger, S. R. Nagel, and R. P. Behringer. Granular solids, liquids, and gases. Rev. Mod. Phys., 68(4):1259-1273, Oct 1996.

J. Javier Brey, F. Moreno, R. García-Rojo, and M. J. Ruiz-Montero. Hydrodynamic Maxwell demon in granular systems. Phys. Rev. E, 65(1):011305, Dec 2001.

J. T. Jenkins and M. W. Richman. Kinetic theory for plane flows of a dense gas of identical, rough, inelastic, circular disks. Physics of Fluids, 28:3485-3494, 1986.

I. Karatzas and S. Shreve. Brownian motion and stochastic calculus. Springer, 1991.

M. Kardar. Statistical physics of fields. Cambridge University Press, 2007.

M. Kardar, G. Parisi, and Y.-C. Zhang. Dynamic Scaling of Growing Interfaces. Phys. Rev. Lett., 56(9):889-892, Mar 1986.

D. Kashchiev. Nucleation: Basic Theory with Applications. Butterworth Heinemann, 2000.

E. Khain and B. Meerson. Onset of thermal convection in a horizontal layer of granular gas. Phys. Rev. E, 67(2):021306, Feb 2003.

Z. S. Khan and S. W. Morris. Subdiffusive Axial Transport of Granular Materials in a Long Drum Mixer. Phys. Rev. Lett., 94(4):048002, Jan 2005. 
H. Kim, L. Guibas, and S. Shin. Efficient Collision Detection among Moving Spheres with Unknown Trajectories. Algorithmica, 43(3):195-210, 2005.

K. Kim, J. Park, J. Moon, H. Kim, and H. Pak. Solid-Liquid Transition in a Highly Dense 3 D Vibro-Fluidized Granular System. Journal of the Korean Physical Society, 40 (6):983-986, 2002.

J. G. Kirkwood. The Statistical Mechanical Theory of Transport Processes I. General Theory. The Journal of Chemical Physics, 14(3):180, 1946.

J. G. Kirkwood. The Statistical Mechanical Theory of Transport Processes II. Transport in Gases. The Journal of Chemical Physics, 15(1):72, 1947.

M. Kohonen, D. Geromichalos, M. Scheel, C. Schier, and S. Herminghaus. On capillary bridges in wet granular materials. Physica A: Statistical Mechanics and its Applications, 339(1-2):7 - 15, 2004. Proceedings of the International Conference New Materials and Complexity.

R. H. Kraichnan. Inertial Ranges in Two-Dimensional Turbulence. Physics of Fluids, to (7):1417-1423, 1967.

R. Kubo. Statistical-Mechanical Theory of Irreversible Processes. I. General Theory and Simple Applications to Magnetic and Conduction Problems. Journal of the Physical Society of Japan, 12(6):570-586, 1957.

A. Kudrolli, M. Wolpert, and J. Gollub. Cluster Formation due to Collisions in Granular Material. Phys. Rev. Lett., 78(7):1383-1386, Feb 1997.

H. Kuninaka and H. Hayakawa. Anomalous Behavior of the Coefficient of Normal Restitution in Oblique Impact. Phys. Rev. Lett., 93(15):154301, Oct 2004.

J. Kurchan. Fluctuation theorem for stochastic dynamics. Journal of Physics A: Mathematical and General, 31(16):3719-3729, 1998.

Y. A. Kuznetsov. Andronov-Hopf bifurcation. Scholarpedia, 1(10):1858, 2006.

B. B. Laird and R. L. Davidchack. Direct Calculation of the Crystal Melt Interfacial Free Energy via Molecular Dynamics Computer Simulation. The Journal of Physical Chemistry $B, 109(38): 17802-17812,2005$.

Y. Lan and A. D. Rosato. Macroscopic behavior of vibrating beds of smooth inelastic spheres. Physics of Fluids, 7(8):1818-1831, 1995.

J. Lebowitz and H. Spohn. A Gallavotti-Cohen-Type Symmetry in the Large Deviation Functional for Stochastic Dynamics. Journal of Statistical Physics, 95(1):333-365, 1999.

B. Legras and B. Villone. Stability of Turbulent Kolmogorov Flow. In J. Peinke, A. Kittel, S. Barth, and M. Oberlack, editors, Progress in Turbulence. Springer Berlin Heidelberg, 2005. 
J. G. Leidenfrost. De Aquae Communis Nonnullis Qualitatibus Tractatus. Duisburg, 1756.

K. Leung and R. K. P. Zia. Geometrically induced transitions between equilibrium crystal shapes. Journal of Physics A: Mathematical and General, 23(20):4593, 1990.

G. Lian, C. Thornton, and M. Adams. A Theoretical Study of the Liquid Bridge Forces between Two Rigid Spherical Bodies. Journal of Colloid and Interface Science, 161(1):138 $-147,1993$.

G. Lian, C. Thornton, and M. J. Adams. Discrete particle simulation of agglomerate impact coalescence. Chemical Engineering Science, 53(19):3381 - 3391, 1998.

C.-C. Liao and S.-S. Hsiau. Experimental analysis of dynamic properties in wet sheared granular matter. Powder Technology, 197(3):222 - 229, 2010.

I. Lifshitz and V. Slyozov. The kinetics of precipitation from supersaturated solid solutions. Journal of Physics and Chemistry of Solids, 19(1-2):35 - 50, 1961.

H. Linke, B. J. Alemán, L. D. Melling, M. J. Taormina, M. J. Francis, C. C. DowHygelund, V. Narayanan, R. P. Taylor, and A. Stout. Self-Propelled Leidenfrost Droplets. Physical Review Letters, 96(15):154502, 2006.

R. Lipowsky. Critical Surface Phenomena at First-Order Bulk Transitions. Phys. Rev. Lett., 49(21):1575-1578, Nov 1982.

E. Lorenz. Deterministic aperiodic flow. J. Atmos. Sci, 20:130-141, 1963.

W. Losert, D. G. W. Cooper, J. Delour, A. Kudrolli, and J. P. Gollub. Velocity statistics in excited granular media. Chaos: An Interdisciplinary Journal of Nonlinear Science, 9 (3):682-690, 1999a.

W. Losert, D. G. W. Cooper, and J. P. Gollub. Propagating front in an excited granular layer. Phys. Rev. E, 59(5):5855-5861, May 1999b.

S. Luding. Towards dense, realistic granular media in 2D. Nonlinearity, 22(12):R101-R146, 2009.

S. Luding, E. Clément, A. Blumen, J. Rajchenbach, and J. Duran. Studies of columns of beads under external vibrations. Phys. Rev. E, 49(2):1634-1646, Feb 1994a.

S. Luding, H. J. Herrmann, and A. Blumen. Simulations of two-dimensional arrays of beads under external vibrations: Scaling behavior. Phys. Rev. E, 50(4):3100-3108, Oct 1994b.

C. Maaß, N. Isert, G. Maret, and C. Aegerter. Experimental Investigation of the Freely Cooling Granular Gas. Physical Review Letters, 100(24):248001, 2008.

M. M. Mansour, C. V. den Broeck, I. Bena, and F. Baras. Spurious diffusion in particle simulations of the Kolmogorov flow. EPL (Europhysics Letters), 47(1):8-13, 1999. 
I. V. Markov. Crystal growth for beginners: fundamentals of nucleation, crystal growth and epitaxy. World Scientific, Singapore, 2003.

C. L. Martin and R. K. Bordia. Influence of adhesion and friction on the geometry of packings of spherical particles. Physical Review E (Statistical, Nonlinear, and Soft Matter Physics), 77(3):031307, 2008.

N. Marwan, M. C. Romano, M. Thiel, and J. Kurths. Recurrence plots for the analysis of complex systems. Physics Reports, 438(5-6):237-329, 2007.

P. Mayor, G. D'Anna, A. Barrat, and V. Loreto. Observing Brownian motion and measuring temperatures in vibration-fluidized granular matter. New Journal of Physics, 7:28, 2005.

J. McDonald. Homogeneous nucleation of vapor condensation. I. Thermodynamic aspects. Am. J. Phys, 3o(870):11, 1962.

S. McNamara and S. Luding. Energy flows in vibrated granular media. Phys. Rev. E, 58 (1):813-822, July 1998.

B. Meerson, T. Pöschel, and Y. Bromberg. Close-Packed Floating Clusters: Granular Hydrodynamics Beyond the Freezing Point? Phys. Rev. Lett., 91(2):024301, Jul 2003.

A. Mehta. Relaxational dynamics, avalanches, and disorder in real sandpiles. In A. Mehta, editor, Granular matter: an interdisciplinary approach. Springer, New York, 1993.

D. Mehta and S. Sahni, editors. Handbook of Data Structures and Applications. Chapman and Hall/CRC, 2004.

F. Melo, P. B. Umbanhowar, and H. L. Swinney. Hexagons, Kinks, and Disorder in Oscillated Granular Layers. Phys. Rev. Lett., 75(21):3838-3841, Nov 1995.

L. Meshalkin and Y. Sinai. Investigation of the stability of a stationary solution of a system of equations for the plane movement of an incompressible viscous liquid. PMM, J. Appl. Math. Mech., 25:1700-1705, 1961.

T. Mikami, H. Kamiya, and M. Horio. Numerical simulation of cohesive powder behavior in a fluidized bed. Chemical Engineering Science, 53(10):1927 - 1940, 1998.

S. Miller and S. Luding. Event-driven molecular dynamics in parallel. J. Comp. Phys., 193(1):306-316, January 2004.

N. Mitarai and F. Nori. Wet granular materials. Advances in physics, 55(1-2):1, 2006.

S. J. Moon, M. D. Shattuck, C. Bizon, D. I. Goldman, J. B. Swift, and H. L. Swinney. Phase bubbles and spatiotemporal chaos in granular patterns. Phys. Rev. E, 65(1):011301, Dec 2001. 
A. Morita. Mean square displacement for Brownian motion under a square-well potential and non-Einstein behaviour. Journal of Physics A: Mathematical and General, 29(20): 6525-6529, 1996.

O. R. Musin. The kissing number in four dimensions. ANNALS OF MATHEMATICS, 1: $1,2008$.

S. R. Nagel. Instabilities in a sandpile. Rev. Mod. Phys., 64(1):321-325, Jan 1992.

E. Nelson. Dynamical Theories of Brownian Motion. Princeton University Press, 1967.

T. Neuhaus and J. Hager. 2 D crystal shapes, droplet condensation, and exponential slowing down in simulations of first-order phase transitions. Journal of Statistical Physics, 113(1): $47-83,2003$.

Y. Norizoe, K. Daoulas, and M. Müller. Measuring excess free energies of self-assembled membrane structures. Faraday Discussions, 144:369-391, 2010.

S. Nowak, A. Samadani, and A. Kudrolli. Maximum angle of stability of a wet granular pile. Nature Physics, 1:50-52, 2005.

S. Ogawa, A. Umemura, and N. Oshima. On the equation of fully fluidized granular flow. J. Appl. Math. Phys., 31:483, 1980.

L. Onsager. Reciprocal Relations in Irreversible Processes. I. Phys. Rev., 37(4):405-426, Feb 1931.

L. Onsager. Crystal Statistics. I. A Two-Dimensional Model with an Order-Disorder Transition. Phys. Rev., 65(3-4):117-149, Feb 1944.

W. Ostwald. On the assumed isomerism of red and yellow mercury oxide and the surface-tension of solid bodies. ZEITSCHRIFT FUR PHYSIKALISCHE CHEMIESTOCHIOMETRIE UND VERWANDTSCHAFTSLEHRE, 34(4):495-503, AUG 1900.

F. Pacheco-Vázquez, G. A. Caballero-Robledo, and J. C. Ruiz-Suárez. Superheating in Granular Matter. Physical Review Letters, 102(17):170601, 2009.

G. Paul. A Complexity $O(1)$ priority queue for event driven molecular dynamics simulations. J. Comp. Phys., 221(2):615-625, February 2007.

P. Perlekar and R. Pandit. Turbulence-induced melting of a nonequilibrium vortex crystal in a forced thin fluid film. New Journal of Physics, 12(2):023033, 2010.

A. Pimpinelli and J. Villain. Physics of crystal growth. Cambridge University Press, 1998.

O. Pitois. Liquid bridge between two moving spheres: An experimental study of viscosity effects. Journal of colloid and interface science, 231(1):26-31, 2000. 
O. Pitois, P. Moucheront, and X. Chateau. Rupture energy of a pendular liquid bridge. The European Physical Journal B, 23(1):79-86, 2001.

N. Platt, L. Sirovich, and N. Fitzmaurice. An investigation of chaotic Kolmogorov flows. Physics of Fluids A: Fluid Dynamics, 3(4):681-696, 1991.

B. Pluis, D. Frenkel, and J. Van der Veen. Surface-induced melting and freezing II. A semi-empirical Landau-type model. Surface Science, 239(3):282-300, 1990.

T. Poeschel and T. Schwager. Computational Granular Dynamics. Springer, October 2004 .

W. H. Press, B. P. Flannery, S. A. Teukolsky, and W. T. Vetterling. Numerical Recipes in C. Cambridge University Press, 2nd edition, October 1992.

A. Prevost, P. Melby, D. A. Egolf, and J. S. Urbach. Nonequilibrium two-phase coexistence in a confined granular layer. Phys. Rev. E, 70(5):050301, Nov 2004.

H. Princen. Comments on the effect of capillary liquid on the force of adhesion between spherical solid particles. Journal of Colloid and Interface Science, 26(2):249 - 253, 1968.

A. Puglisi, M. Assaf, I. Fouxon, and B. Meerson. Attempted density blowup in a freely cooling dilute granular gas: Hydrodynamics versus molecular dynamics. Physical Review E (Statistical, Nonlinear, and Soft Matter Physics), 77(2):021305, 2008.

D. Quere and A. Ajdari. Liquid drops: Surfing the hot spot. Nature Materials, 5(6): 429-430, 062006.

S. H. E. Rahbari. Solid-Fluid Transitions in Wet Granular Material. PhD thesis, University Goettingen, 2009.

S. H. E. Rahbari, J. Vollmer, S. Herminghaus, and M. Brinkmann. A response function perspective on yielding of wet granular matter. EPL (Europhysics Letters), 87(1):14002, 2009.

R. Ramírez, T. Pöschel, N. Brilliantov, and T. Schwager. Coefficient of restitution of colliding viscoelastic spheres. Phys. Rev. E, 6o(4):4465-4472, Oct 1999.

J. Rammer. Quantum Field Theory of Non-equilibrium States. Cambridge University Press, 2007.

R. Reichardt and W. Wiechert. Event driven algorithms applied to a high energy ball mill simulation. Granular Matter, 9(3):251-266, June 2007.

P. M. Reis, R. A. Ingale, and M. D. Shattuck. Crystallization of a Quasi-Two-Dimensional Granular Fluid. Physical Review Letters, 96(25):258001, 2006. 
F. V. Reyes and J. S. Urbach. Effect of inelasticity on the phase transitions of a thin vibrated granular layer. Physical Review E (Statistical, Nonlinear, and Soft Matter Physics), 78 (5):051301, 2008.

L. F. Richardson. Atmospheric Diffusion Shown on a Distance-Neighbour Graph. Proceedings of the Royal Society of London. Series A, Containing Papers of a Mathematical and Physical Character, 110(756):709-737, 1926.

G. H. Ristow. Critical exponents for granular phase transitions. EPL (Europhysics Letters), 40(6):625-630, 1997.

R. Robert and C. Rosier. Long range predictability of atmospheric flows. Nonlinear processes in geophysics, 8(1/2):55-67, 2001.

G. Robinson and M. Thiel. Recurrences determine the dynamics. Chaos: An Interdisciplinary Journal of Nonlinear Science, 19(2):023104, 2009.

K. Roeller. Granulardynamik - Simulationen zur Fluidisierungsdynamik bei vertikaler Agitation. master thesis, University Ulm, 2005.

K. Roeller, J. Vollmer, and S. Herminghaus. Unstable Kolmogorov flow in granular matter. Chaos: An Interdisciplinary Journal of Nonlinear Science, 19(4):041106, 2009.

F. Rouyer and N. Menon. Velocity Fluctuations in a Homogeneous 2D Granular Gas in Steady State. Phys. Rev. Lett., 85(17):3676-3679, Oct 2000.

D. Ruelle and F. Takens. On the nature of turbulence. Communications in Mathematical Physics, 20(3):167-192, 1971.

T. Rump. Coarsening processes in thin liquid films: Analysis and numerics. PhD thesis, University Bonn, 2008.

H. Rumpf. The strength of granules and agglomerates. In W. Knepper, editor, Agglomeration, page 379, New York, 1962. Wiley Interscience.

L. Santen and W. Krauth. Liquid, Glass and Crystal in Two-dimensional Hard disks, 2001.

T. Sasamoto and H. Spohn. Universality of the One-dimensional KPZ Equation. Arxiv preprint arXiv:1002.1883, 2010.

M. Scheel, D. Geromichalos, and S. Herminghaus. Wet granular matter under vertical agitation. Journal of Physics: Condensed Matter, 16(38):S4213-S4218, 2004.

M. Scheel, R. Seemann, M. Brinkmann, M. Di Michiel, A. Sheppard, B. Breidenbach, and S. Herminghaus. Morphological clues to wet granular pile stability. Nature Materials, 7(3):189-193, 2008. 
B. Schulz and M. Schulz. The dynamics of wet granular matter. Journal of Non-Crystalline Solids, 352(42-49):4877-4879, 2006. Proceedings of the 5th International Discussion Meeting on Relaxations in Complex Systems, 5th International Discussion Meeting on Relaxations in Complex Systems.

M. Schulz, B. M. Schulz, and S. Herminghaus. Shear-induced solid-fluid transition in a wet granular medium. Phys. Rev. E, 67(5):052301, May 2003.

T. Schwager. Coefficient of restitution for viscoelastic disks. Physical Review E (Statistical, Nonlinear, and Soft Matter Physics), 75(5):051305, 2007.

T. Schwager and T. Pöschel. Coefficient of normal restitution of viscous particles and cooling rate of granular gases. Phys. Rev. E, 57(1):650-654, Jan 1998.

P.-J. Sell and A. W. Neumann. The Surface Tension of Solids. Angezwandte Chemie International Edition in English, 5(3):299-307, 1966.

D. Sharp. Overview of Rayleigh-Taylor instability. In Fronts, interfaces and patterns conference, volume 2, 1983 .

Z. S. She. Metastability and vortex pairing in the kolmogorov flow. Physics Letters A, 124 (3):161 - 164, 1987.

R. Shuttleworth. The Surface Tension of Solids. Proceedings of the Physical Society. Section A, 63(5):444, 1950 .

S. J. R. Simons and R. J. Fairbrother. Direct observations of liquid binder-particle interactions: the role of wetting behaviour in agglomerate growth. Powder Technology, 110(1-2): $44-58,2000$.

R. Son, J. Perez, and G. Voth. Experimental measurements of the collapse of a twodimensional granular gas under gravity. Physical Review E (Statistical, Nonlinear, and Soft Matter Physics), 78(4):041302, 2008.

R. Soto, M. Mareschal, and D. Risso. Departure from Fourier's Law for Fluidized Granular Media. Phys. Rev. Lett., 83(24):5003-5006, Dec 1999.

T. Speck and U. Seifert. Distribution of work in isothermal nonequilibrium processes. Phys. Rev. E, 70(6):066112, Dec 2004.

I. Stewart. Mathematics: The Lorenz attractor exists. Nature, 406(6799):948-949, Aug 2000.

D. Sumpter. The principles of collective animal behaviour. Philosophical Transactions of the Royal Society B: Biological Sciences, 361(1465):5-22, 2006.

N. Taberlet, P. Richard, J. T. Jenkins, and R. Delannay. Density inversion in rapid granular flows: the supported regime. The European Physical Journal E, 22(1):17-24, 2007. 
W. Tiller. The Science of Crystallization: Microscopic Interfacial Phenomena. Cambridge University Press, 1991.

S. Torquato, T. M. Truskett, and P. G. Debenedetti. Is Random Close Packing of Spheres Well Defined? Phys. Rev. Lett., 84(10):2064-2067, Mar 2000.

W. Tucker. The Lorenz attractor exists. Comptes Rendus de l'Academie des Sciences, 328 (12):1197-1202, 1999.

S. Ulrich, T. Aspelmeier, K. Roeller, A. Fingerle, S. Herminghaus, and A. Zippelius. Cooling and Aggregation in Wet Granulates. Physical Review Letters, 102(14):148002, 2009a.

S. Ulrich, T. Aspelmeier, A. Zippelius, K. Roeller, A. Fingerle, and S. Herminghaus. Dilute wet granular particles: Nonequilibrium dynamics and structure formation. Physical Review E (Statistical, Nonlinear, and Soft Matter Physics), 80(3):031306, $2009 \mathrm{~b}$.

P. Umbanhowar and M. van Hecke. Force dynamics in weakly vibrated granular packings. Phys. Rev. E, 72(3):030301, Sep 2005.

P. B. Umbanhowar, F. Melo, and H. L. Swinney. Localized excitations in a vertically vibrated granular layer. Nature, 382:793-796, 1996.

M. Urbakh, J. Klafter, D. Gourdon, and J. Israelachvili. The nonlinear nature of friction. Nature, 430(6999):525-528, 2004.

J. M. Valverde, M. A. S. Quintanilla, and A. Castellanos. Jamming Threshold of Dry Fine Powders. Phys. Rev. Lett., 92(25):258303, Jun 2004.

K. van der Weele, D. van der Meer, M. Versluis, and D. Lohse. Hysteretic clustering in granular gas. EPL (Europhysics Letters), 53(3):328-334, 2001.

T. van Noije. Kinetic and Mesoscopic Theories of Granular Fluids. PhD thesis, University Utrecht, 1999.

T. van Noije and M. H. Ernst. Velocity Distributions in Homogeneously Cooling and Heated Granular Fluids. arXiv.org:cond-mat, 9803042, 1998.

C. P. Verdon, R. L. McCrory, R. L. Morse, G. R. Baker, D. I. Meiron, and S. A. Orszag. Nonlinear effects of multifrequency hydrodynamic instabilities on ablatively accelerated thin shells. Physics of Fluids, 25(9):1653-1674, 1982.

R. Vink and J. Horbach. Critical behaviour and interfacial fluctuations in a phaseseparating model colloidEndash;polymer mixture: grand canonical Monte Carlo simulations. Journal of Physics: Condensed Matter, 16(38):S3807-3820, 2004.

R. L. C. Vink, J. Horbach, and K. Binder. Capillary waves in a colloid-polymer interface. The Journal of Chemical Physics, 122(13):134905, 2005. 
P. Visco, A. Puglisi, A. Barrat, E. Trizac, and F. van Wijland. Injected power and entropy flow in a heated granular gas. EPL (Europhysics Letters), 72(1):55-61, 2005.

H. Vogel. Gerthsen Physik. Springer, 20. edition, 1999.

J. Vollmer, A. G. Vegh, C. Lange, and B. Eckhardt. Vortex formation by active agents as a model for Daphnia swarming. Physical Review E (Statistical, Nonlinear, and Soft Matter Physics), 73(6):061924, 2006.

M. von Smoluchowski. Zur kinetischen Theorie der Brownschen Molekularbewegung und der Suspensionen. Ann. d. Physik, 21:756-780, 1906.

P. W. Voorhees. Ostwald Ripening of Two-Phase Mixtures. Annual Review of Materials Science, 22(1):197-215, 1992.

J. Walker. Essay 10: Boiling and the Leidenfrost Effect. In D. Halliday and R. Resnick, editors, Fundamentals of Physics, pages E10-1 - 10-6. John Wiley \& Sons, 3rd edition, 1988. www.wiley.com/college/phy/halliday320005/pdf/leidenfrost_essay.pdf.

S. Warr, J. Huntley, and G. Jacques. Fluidization of a two-dimensional granular system: Experimental study and scaling behavior. Phys. Rev. E, 52(5):5583-5595, Nov 1995.

R. D. Wildman and D. J. Parker. Coexistence of Two Granular Temperatures in Binary Vibrofluidized Beds. Phys. Rev. Lett., 88(6):064301, Jan 2002.

C. Willett, M. Adams, S. Johnson, and J. Seville. Capillary Bridges between Two Spherical Bodies. Langmuir, 16(24):9396-9405, 2000.

K. G. Wilson and J. Kogut. The renormalization group and the $\varepsilon$ expansion. Physics Reports, 12(2):75 - 199, 1974.

N. Xu and C. O'Hern. Effective Temperature in Athermal Systems Sheared at Fixed Normal Load. Phys. Rev. Lett., 94(5):055701, 2005.

J. M. Yeomans. Statistical Mechanics of Phase Transitions. Oxford University Press, 1992.

J. Yvon. Theorie Statistique des Fluides et l'Equation d'Etat. Actes sientifique et industrie, 203, 1935 .

V. Zaburdaev and S. Herminghaus. Dry and wet granular shock waves. Physical Review E (Statistical, Nonlinear, and Soft Matter Physics), 75(3):031304, 2007.

V. Zaburdaev, M. Brinkmann, and S. Herminghaus. Free Cooling of the OneDimensional Wet Granular Gas. Physical Review Letters, 97(1):018001, 2006.

A. Zhabotinsky. Periodic processes of the oxidation of malonic acid in solution (Study of the kinetics of Belousov's reaction). Biofizika, 9:306-311, 1964. 
A. Zhabotinsky. Belousov-Zhabotinsky reaction. Scholarpedia, 2(9):1435, 2007.

X. Zhang and A. L. Frenkel. Large-Scale Instability of Generalized Oscillating Kolmogorov Flows. SIAM Journal on Applied Mathematics, 58(2):540-564, 1998.

H. Zhu and A. Yu. A theoretical analysis of the force models in discrete element method. Powder Technology, 161(2):122 - 129, 2006.

H. Zhu, Z. Zhou, R. Yang, and A. Yu. Discrete particle simulation of particulate systems: Theoretical developments. Chemical Engineering Science, 62(13):3378 - 3396, 2007. Frontier of Chemical Engineering - Multi-scale Bridge between Reductionism and Holism.

H. Zhu, Z. Zhou, R. Yang, and A. Yu. Discrete particle simulation of particulate systems: A review of major applications and findings. Chemical Engineering Science, 63(23):5728 $-5770,2008$. 


\section{List of publications}

A. Fingerle, K. Roeller, K. Huang, and S. Herminghaus. Phase transitions far from equilibrium in wet granular matter. New Journal of Physics, 10(5):053020 (10pp), 2008

S. Ulrich, T. Aspelmeier, K. Roeller, A. Fingerle, S. Herminghaus, and A. Zippelius. Cooling and Aggregation in Wet Granulates. Physical Review Letters, 102(14):148002, 2009a

S. Ulrich, T. Aspelmeier, A. Zippelius, K. Roeller, A. Fingerle, and S. Herminghaus. Dilute wet granular particles: Nonequilibrium dynamics and structure formation. Physical Review E (Statistical, Nonlinear, and Soft Matter Physics), 80(3):031306, 2009b

K. Roeller, J. Vollmer, and S. Herminghaus. Unstable Kolmogorov flow in granular matter. Chaos: An Interdisciplinary Journal of Nonlinear Science, 19(4):041106, 2009

K. Huang, K. Roeller, and S. Herminghaus. Universal and non-universal aspects of wet granular matter under vertical vibration. submitted to EJP, 2009 


\section{Zusammenfassung in deutscher Sprache}

Feuchte granulare Materie kann als ein nützliches System dienen, um die Dynamik zu untersuchen, die bei Systemen im thermischen Ungleichgewicht entsteht. Es ist sowohl durch Experimente als auch durch Simulationen sehr leicht zugänglich. In der vorliegenden Dissertation wurde feuchte granulare Materie mit Hilfe von zeitals auch ereignisgetriebenen Molekulardynamik Simulationen untersucht. Dabei wurde die Energiezufuhr, die nötig ist, um das System aus dem thermischen Gleichgewicht zu bringen, durch zwei höchst unterschiedliche sinusförmige Antriebsmechanismen realisiert. Während in den Kapiteln 3 bis 6 das sinusförmige Schütteln verwendet wurde, war in den Kapitel 7 und 8 das sinusförmige Scheren die treibende Kraft. Die Wechselwirkungen mit der Flüssigkeit im System wurde durch das Minimale Kapillar bzw. durch das Bindfadenmodell realisiert. Diese sorgen für den Energieaustrag aus dem System, sodass das System einen stationären Zustand erreichen kann.

Im ersten Teil der vorliegenden Arbeit wurde sinusförmiges Schütteln verwendet. Dies ermöglicht es dem System, sich in einem homogenen Zustand wiederzufinden der sehr and die Phasen fest, flüssig und gasförmig erinnert, die aus dem thermodynamischen Gleichgewicht bekannt sind. In Anlehnung an die aus thermodynamischen Gleichgewichtssystemen bekannten Phasendiagramme wurden in dieser Arbeit Phasendiagramme sowohl für zwei- als auch für dreidimensionale feuchte Granulate erstellt. Anhand von Simulationen konnte gezeigt werden, dass geschütteltes feuchtes Granulat zwei universelle Phasenübergänge aufweist, die lediglich von wenigen System-Parametern abhängen. Einer dieser beiden Phasenübergänge ist der fest-flüssig Übergang, der, wie sich herausstellte, vor allem durch die Antriebkraft hervorgerufen wurde, wobei die Antriebsenergie nur eine Korrektur zweiter Ordnung darstellt. Ein einfaches theoretisches Model, das nur das Gleichgewicht der wesentlichen Kräfte im System annimmt, zeigt eine gute qualitative Übereinstimmung mit den Simulationen bei geringen Antriebsenergien. Das Model legt zugrunde, dass die mittlere freie Weglänge zwischen den Schichten der Teilchen und dem kritischen Abreißabstand, an dem die Kapillarbrücken reißen, den Übergang von fest zu flüssig bestimmt. Desweiteren prognostiziert das Model ein Oberflächenschmelzen, das ebenfalls in Simulationen beobachtet werden konnte. Der zweite Phasenübergang im feuchten Granulat findet zwischen der flüssigen und der gasförmigen Phase statt, der mit einem breiten Koexistenz Bereich einhergeht. Dieser 
Übergang wird durch die maximale kinetische Energie der antreibenden Wand hervorgerufen und tritt ein sobald die maximale Energie, die durch einen einzigen Zusammenstoß mit der Wand geliefert wird, ausreichend ist um eine der Kapillarbrücken zu zerreißen. Zudem wurde noch ein weiterer Übergang zwischen einer festen und einer flüssigen Phase gefunden der anscheinend durch die Reduzierung der Polydispersität hervorgerufen wird. Dieser Übergang ist ebenfalls ein energiegetriebener Übergang und wird von einer fest-flüssig Koexistenz begleitet.

Die Untersuchung der Größe der Gasblase, die sich im flüssig-gas Koexistenzbereich bildet, zeigte drei verschiedene Formen auf: eine runde Gasblase, umgeben von Flüssigkeit; eine streifenförminge Anordnung und ein flüssiger Tropfen umgeben von einem Gas. Die Situation erinnert an die Übergänge zwischen verschiedenen Formen, die in einem zweidimensionalen Ising Modell beobachtet werden können. Der Übergang zwischen den beiden Formen ist dort ein Phasenübergang erster Ordnung und wird durch die Minimierung der Grenzflächenenergie angetrieben. Diese Ähnlichkeit zum Ising Modell führte zu der Annahme, dass auch im feuchten Granulat eine Größe ähnlich einer Grenzflächenenergie existieren könnte. Diese Grenzflächenenergie wurde in Simulationen durch die mechanische Deformierung der Grenzfläche bestimmt, und gefunden, dass sie in guter qualitativer Übereinstimmung mit einer einfachen Abschätzungen war. Weitere Untersuchungen der Allgemeingültigkeit dieser Grenzflächenspannung zeigten in den dreidimensionalen Simulationen der fest-flüssig Koexistenz ein Verhalten, das an die Ostwald-Reifung erinnert, welche bekanntermaßen durch die Minimierung freien Grenzflächenengergie angetrieben wird. Durch diese Ähnlichkeit der beiden beschriebenen Effekte wird hier angenommen, dass die Grenzflächenenergie auch in der fest-flüssigen Koexistenz im dreidimensionalen System auftritt.

Die Koexistenz kann nicht nur in horizontaler, sondern auch in vertikaler Richtung auftauchen. Dies ließ eine Dichteinversion vermuten, die wiederum an den Leidenfrost-Effekt, bekannt von klassischen Flüssigkeiten, erinnert. Die offensichtliche Reduzierung der Wärmeleitfähigkeit resultiert in der klaren Grenzfläche zwischen dem heißen Gas und dem kalten kondensierten Pfropfen, der oberhalb des Gases schwebt. Der Pfropfen vollführt eine gleichmäßige Oszillation, deren Frequenz mit Hilfe eines einfachen theoretischen Models bestimmt werden kann. Es handelt sich dabei um die gleiche Oszillationsfrequenz wie man sie im Fall eines passiven Pfropfens erwarten würde, der über einem realen Gas schwebt, das um das ausgeschlossene Volumen korrigiert wurde. Das Hinzufügen eines lateralen Teilchenaustauschs führt zu einem Effekt der stark an eine Rayleigh-Taylor Instabilität erinnert. Dieses gesamte Szenario ist sehr ähnlich zum Leidenfrost Effekt den man z. B. bei Wasser beobachtet. Dieser Zusammenhang wird auch in den Simulationen beobachtet und gut durch das theoretische Modell vorhergesagt.

Im zweiten Teil der vorliegenden Arbeit wurde das Granulat sinusförmig geschert. Wiederum wurden zuerst die Phasendiagramme gezeigt, die einen festen sowie einen fluidisierten Zustand unterscheiden. Außerdem wurde ein einfaches theoretisches Model dargestellt, das die Stabilität einer eindimensionalen Kette von Teilchen 
mit dem in den Simulationen beobachteten Übergang verknüpfte. Dabei konnte eine gute qualitative Übereinstimmung zwischen der theoretischen Berechnung und der Simulation festgestellt werden. Die Grenzen des theoretischen Models wurden klar diskutiert und mögliche Gründe für Abweichungen aufgezeigt. Darauf aufbauend folgte eine Studie der reichhaltigen und komplexen Dynamik, die im fluidisierten Zustand auftritt. Es wurden dynamischen Instabilitäten gefunden die mit den Methoden der Nichtlinearen Dynamik untersucht wurden. Dabei wurden drei verschiedene Zustände entdeckt. Im ersten, osziallatorischen Bereich wird das Scherband periodisch instabil und es bilden sich Wirbel im System. Bei größeren Antriebskräften wurde ein Bereich entdeckt in dem diese Wirbel stationär sind. Dieser stationäre Zustand wird bei noch größeren Antriebskräften instabil und zeigt periodische Größenänderungen der Löcher die in diesem System enthalten sind. Die Dynamik des Systems wurde auf einen zweidimensionalen Phasenraum reduziert und die Grenzzyklen untersucht, die auf eine Hopfbifurkation beim Übergang zwischen den einzelnen Bereichen hindeuteten. Daraus wurde auch die Wiederkehrabbildung erstellt.

Zusammenfassend lässt sich sagen, dass eine große Anzahl an Simulationen zu feuchten Granulaten in der vorliegenden Arbeit durchgeführt wurden. Ermöglicht wurde dies vor allem durch das bemerkenswert einfache Model (das minimale Kapillarmodell), das zur Beschreibung der Kapillar-Wechselwirkungen genutzt wird. Eine Abwandelung dieses Models (Bindfaden Modell) ermöglichte einen bedeutenden Anstieg der Leistung in der Simulation. Desweiteren wurde eine erstaunliche Bandbreite von dynamischem Verhalten in den feuchten Granulaten entdeckt. In vielen Fällen konnten einfach theoretische Modelle aufgestellt werden, die zwar nur einen kleinen Teil der komplexen Problematik berücksichtigt, aber dennoch erstaunlich gute Vorhersagen treffen kann. Trotz der Einfachheit der verwendeten Modelle konnten in vielen Fällen gute qualitative Übereinstimmungen zwischen den Simulationen und den Experimenten ausgemacht werden.

Viele Phänomene, die in den Simulationen zu feuchten Granulaten beobachtet wurden, erinnerten stark an das bekannte Verhalten von klassischen Fluiden. Dadurch wird die Aussage berechtigt, dass feuchte granulare Materie ein komplexes Fluid ist; komplex zwar, aber dennoch ein Fluid. 


\section{Acknowledgements}

It is a pleasure to thank the many people who made this thesis possible. I would like to express my deep gratitude to my supervisor, Professor Dr. S. Herminghaus. His breadth of knowledge and his logical way of thinking have been of great value to me. With his enthusiasm, his inspiration, and his consistent efforts to explain things clearly, he provided a very good basis for the present thesis. Likewise I would like to thank the reviewer of the present thesis, Prof. Dr. A. Zippelius. I like to thank Prof. Dr. J. Vollmer who accompanied me on the sinusoidal shearing projects. It was always a pleasure to discuss new ideas with him and who encouraged me with detailed and constructive comments throughout my research and thesis-writing period. I would also like to thank Prof. F. van Wijland for the cooperation with the calculation of the velocity distribution function. I am especially grateful to Dr. K. Huang who performed many experiments and supplied much important data to this thesis. I would like to extend my warm thanks to Dr. S. Ulrich for the cooperation on the free cooling of a wet granular gas. I thank Dr. A. Hager-Fingerle for the cooperation on the phase diagram, his help to translate my time-driven molecular dynamics simulation program from FORTRAN to $\mathrm{C}$ and introducing me to the event-driven simulation technique. I am indebted to my research team colleagues for providing a stimulating and pleasant atmosphere. Our extensive discussions regarding my research and their support in various issues greatly encouraged me. Especially, I would like to thank J. O. Claussen with whom I had many discussions about physics but also about information technologies. Special thanks also goes to J. Blaschke who assisted me with his knowledge on Mathematica whenever I needed him. I also had many intersting discussions with Dr. M. Scheel, Dr. M. Brinkmann and Dr. M. Schröter. A very special thanks to S. Deffner, whom I met during my research months in Paris. We spent many hours, sometimes until late night, discussing interesting issues concerning physics. I am grateful to my girlfriend Katharina who is always there if I need her help. Thank you.

My sincere thanks to all the proofreaders of the present thesis who donated their valuable time and effort despite the DPG conference. J. Blaschke, F. van Bussel, J. O. Claussen, K. Delius, Dr. H. Evans, Dr. Z. Khan, V. Pernice, S. Pielawa, Dr. M. Schröter, S. Thutupalli, S. Uppaluri, S. Utermann and Prof. Dr. J. Vollmer. I also gratefully acknowledge the IT staff at the MPI for giving me all of the necessary assistance during my studies and maintaining the technical systems with great 
knowledge. I wish to express my warmest appreciation for the constant support of my parents, brother, and sister throughout this period.

Finally, I would like to thank the Studienstifung des Deutschen Volkes for their financial support, enabling me to attend various conferences where I presented my work and received constructive feedback from other participants. 


\title{
Awards $^{2}$ and scientific meetings
}

\author{
Awards
}

2007

Heinz-Billing-Preis zur Förderung des wissenschaftlichen Rechnens

(together with A. Fingerle)

for the work "Efficient Simulation Techniques for Dry and Wet Granular Matter"

(see appendix $\bar{B}$ )

awarded by the Heinz-Billing-Foundation of the Max Planck Society

For more information: http://www.billingpreis.mpg.de/

2009

Image Gallery Award

for the work "Unstable Kolmogorov flow in granular matter" (cf. chapter 8)

awarded by the Group of Statistical \& Nonlinear Physics of the American Physical Society

For more information: http://www.aps.org/units/gsnp/gallery/

\section{Scientific meetings}

2007 DPG Frühjahrstagung, Regensburg (posters)

Max Planck PhDnet Workshop, Frankfurt/Main (poster)

Trimester: Statistical physics out of equilibrium, Paris

2008 DPG Frühjahrstagung, Berlin (poster)

Doktorandenforum der Studienstiftung des deutschen Volkes, Köln (talk)

2009 DPG Frühjahrstagung, Dresden (talk)

Doktorandenforum der Studienstiftung des deutschen Volkes, Berlin (talk)

Dynamic days Europe, Göttingen (poster)

Doktorandenforum der Studienstiftung des deutschen Volkes, Koppelsberg

2010

DPG Frühjahrstagung, Regensburg (talk) 



\section{Curriculum Vitae}

\section{Persönliche Angaben}

$\begin{array}{ll}\text { Name } & \text { Klaus Walter Röller } \\ \text { Geburtstag } & \text { 20. März 1982 } \\ \text { Geburtsort } & \text { Ehingen } \\ \text { Staatsangehörigkeit } & \text { deutsch }\end{array}$

\section{Ausbildung}

Aug. 2006 - heute Promotionsstudium, Max-Planck-Institut für Dynamik und Selbstorganisation, Göttingen.

Sep. 2007 - Dez. 2007 Trimester: Statistical physics out of equilibrium L'Institut Henri Poincaré, Paris.

Okt. 2001 - Aug. 2005 Studium der Physik und Wirtschaftsphysik, Universität Ulm. Abschluss jeweils mit Diplom.

Sep. 1992 - Jun. 2001 Allgemeine Hochschulreife, Kreisgymnasium Riedlingen.

\section{Berufserfahrung}

Okt. 2005 - Jun. 2006 Praktikum bei der Siemens Ltd., Nigeria.

Okt. 2003 - Mär. 2009 Verschiedene Tätigkeiten als wissenschaftliche Hilfskraft an den Universitäten Ulm und Göttingen (Mathematik, Physik).

Mär. 2002 \& Aug. 2002 Werksstudent im DaimlerChrysler Forschungszentrum Ulm.

\section{Stipendium}

Jan. 2008 - Jul. 2009 Promotionsstipendiat der Studienstiftung des deutschen Volkes. 